\title{
On the Predictable Representation Property of Martingales Associated with Lévy Processes
}

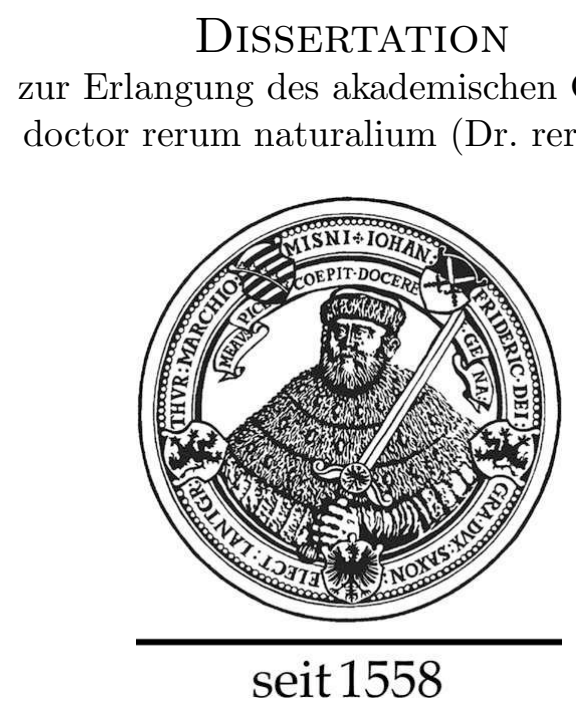

vorgelegt dem Rat

der Fakultät für Mathematik und Informatik der Friedrich-Sciller-Universität Jena

von M. Sc. Paolo Di Tella geboren am 3.3.1984 in Rom 


\section{Gutachter}

1. Prof. Dr. Hans-Jürgen Engelbert (summa cum laude)

2. Prof. Dr. Yuri Kabanov (summa cum laude)

3. Prof. Dr. Ilya Pavlyukevich (summa cum laude)

Tag der öffentlichen Verteidigung: 14. Juni 2013 
A memoria del piccolo Vincenzo.

A mia sorella Marta e a zio Paolo. 

Acknowledgments vii

Zusammenfassung ix

Abstract $\quad$ xi

Introduction $\quad$ xiii

1. Preliminaries 1

1.1. Measure Theory . . . . . . . . . . . . . . . . . . . 1

1.1.1. Measurability and Integration . . . . . . . . . . . . . . . 1

1.1.2. Monotone Class Theorems . . . . . . . . . . . . . . . . 4

1.1.3. Total Systems . . . . . . . . . . . . . . . . 5

1.2. Basics of Stochastic Processes . . . . . . . . . . . . . 6

1.2.1. Filtrations and Stopping Times . . . . . . . . . . 8

1.2.2. Martingales . . . . . . . . . . . . . . . . . . 10

1.2.3. Increasing Processes . . . . . . . . . . . . . . . 11

1.2.4. The Poisson process . . . . . . . . . . . . . . 14

1.2.5. Locally Square Integrable Martingales . . . . . . . . . . . 15

1.2.6. The Wiener process . . . . . . . . . . . . . . . 15

1.2.7. Orthogonality of Local Martingales . . . . . . . . . . . . 16

1.2.8. Semimartingales . . . . . . . . . . . . . . . 17

1.3. Spaces of Martingales and Stochastic Integration . . . . . . . . . . 19

1.3.1. Spaces of Martingales . . . . . . . . . . . . . . . . 19

1.3.2. Stochastic Integral for Local Martingales . . . . . . . . . . . . 22

1.3.3. Stochastic Integral for Semimartingales . . . . . . . . . 25

1.4. Stable Subspaces of Martingales . . . . . . . . . . . . . . . . . 27 
2. Poisson Random Measures and Lévy Processes 33

2.1. Additive Processes and Lévy Processes . . . . . . . . . . . . . . . . . 33

2.2. Poisson Random Measures . . . . . . . . . . . . . . . . . . . . . 37

2.2.1. The Jump Measure of a càdlàg Process . . . . . . . . . . . . 37

2.2.2. Definition of Poisson Random Measures . . . . . . . . . . . . 39

2.2.3. The Stochastic Integral for Poisson Random Measures . . . . 41

2.2.4. Compensated Poisson Random Measures . . . . . . . . . . 44

2.2.5. Construction of Lévy Processes . . . . . . . . . . . . . . 47

2.3. Generalities on Lévy Processes _. . . . . . . . . . . . . . . 51

2.3.1. The Jump Measure of a Lévy Process . . . . . . . . . . . . 51

2.3.2. Itô-Lévy Decomposition and Moments . . . . . . . . . . . . 56

2.3.3. The Structure of the Natural Filtration of a Lévy Process . . 58

3. Martingale Problems for Lévy Processes 61

3.1. The Gaussian Part . . . . . . . . . . . . . . . . . . . 62

3.2. The Jump Measure . . . . . . . . . . . . . . . . . . 63

3.3. Uniqueness of the Solution of the Martingale Problem . . . . . . . 67

3.4. Representation of Martingales . . . . . . . . . . . . . . . . . 68

3.5. Square Integrable Lévy Processes . . . . . . . . . . . . . . . . 71

3.6. Open Problems . . . . . . . . . . . . . . . . . . . . . 74

3.6.1. Characterization of the Predictable Representation Property 74

3.6.2. A Stronger Representation . . . . . . . . . . . . . 75

4. Representation of Square Integrable Martingales 81

4.1. Compensated-Covariation Stable Families . . . . . . . . . . . . . . . 81

4.1.1. Continuous Gaussian Families . . . . . . . . . . . . . . 97

4.1.2. Independent Families of Poisson Processes . . . . . . . . . . . 99

4.2. Martingales of the Natural Filtration of a Lévy Process . . . . . . . 100

4.2.1. Complete Orthogonal Systems . . . . . . . . . . . . . 105

4.2.2. Square Integrable Lévy Processes . . . . . . . . . . . . . . . 108

5. Examples 111

5.1. Compensated Poisson Processes . . . . . . . . . . . . . . . . . 111

5.2. Teugels Martingales . . . . . . . . . . . . . . . . . . . . 112

5.3. Lévy Measures Equivalent to the Lebesgue Measure . . . . . . . . . 115

5.3.1. Hermite Polynomials . . . . . . . . . . . . . . . . . 116

5.3.2. Haar Wavelet . . . . . . . . . . . . . . . . . . . 117

A. Complement to Additive Processes 119

A.1. Independence of Additive Processes . . . . . . . . . . . . . . . . 119

A.2. Itô-Lévy Decomposition . . . . . . . . . . . . . . . . 128

$\begin{array}{ll}\text { B. Denseness of Polynomials } & 135\end{array}$

$\begin{array}{ll}\text { Bibliography } & 139\end{array}$ 


\section{Symbols and Acronyms}

a.e. almost everywhere

a.s. almost surely

càdlàg continu á droite, limitè á gauche

cf. compare (abbreviation of Latin confer)

e.g. for example (abbreviation of Latin exempli gratia)

i.e. that is (abbreviation of Latin $i d$ est)

p.d. pairwise disjoint

PRP predictable representation property

resp. respectively

\section{Norms and Numerical Sets}

$\begin{array}{llll}\text { Symbol } & \text { Page } & \text { Symbol } & \text { Description } \\ \|\cdot\|_{q},\|\cdot\|_{L^{q}(\mu)} & 3 & \mathbb{N} & \text { natural numbers } \\ \|\cdot\|_{\mathscr{H}^{q}} & 19 & \mathbb{Q} & \text { rational numbers } \\ \|\cdot\|_{B M O} & 20 & \mathbb{R} & \text { real numbers } \\ \|\cdot\|_{q}^{*} & 19 & \mathbb{R}_{+} & \text {nonnegative real numbers } \\ \|\cdot\|_{L^{q}(M)}^{*} & 22 & \mathbb{Z} & \text { integer numbers }\end{array}$

\section{Classes of Processes}

$\begin{array}{lll}\text { Symbol } & \text { Description } & \text { Page } \\ \mathscr{A}^{+} & \text {integrable increasing processes } & 13 \\ \mathscr{A}^{+} & \text {processes with integrable variation } & 13 \\ \mathscr{A}_{\text {loc }}^{+} & \text {locally integrable increasing processes } & 13 \\ \mathscr{A}_{\text {loc }} & \text { processes with locally integrable variation } & 13 \\ B M O & B M O \text {-martingales } & 20 \\ B M O_{0} & B M O \text {-martingales starting at } 0 & 20 \\ \mathscr{C}_{\text {loc }} & \text { localized class } \mathscr{C} & 9 \\ \mathscr{H}^{q} & q \text {-integrable martingales } & 19 \\ \mathscr{H}_{0}^{q} & q \text {-integrable martingales starting at } 0 & 19 \\ \mathscr{H}_{\text {loc }} & \text { localization of } \mathscr{H}^{q} & 19 \\ \mathscr{H}_{\text {loc }, 0}^{q} & \text { localization of } \mathscr{H}_{0}^{q} & 19 \\ \mathrm{~K} & \text { integrands for semimartingales } & 25 \\ \mathscr{L}^{q}(\mathscr{X}) & \text { stable subspace generated in } \mathscr{H}_{0}^{q} \text { by } \mathscr{X} & 28 \\ \mathrm{~L}^{q}(M) & \text { predictable integrands of order } q \text { for } M & 22 \\ \mathrm{~L}_{\text {loc }}^{q}(M) & \text { predictable integrands locally of order } q \text { for } M & 22 \\ \mathscr{M} & \text { uniformly integrable martingales } & 10 \\ \mathscr{M}_{0} & \text { uniformly integrable martingales starting at } 0 & 10 \\ \mathscr{M}_{\text {loc }} & \text { local martingales } & 11 \\ \mathscr{M}_{\text {loc }, 0} & \text { local martingales starting at } 0 & 11 \\ \mathscr{M}_{\text {loc }}^{c} & \text { continuous local martingales } & 16 \\ \mathscr{M}_{\text {loc }}^{d} & \text { purely discontinuous local martingales } & 16 \\ \mathscr{V}^{+} & \text {increasing processes } & 11 \\ \mathscr{V} & \text { processes of finite variation } & 11\end{array}$




\section{Other symbols}

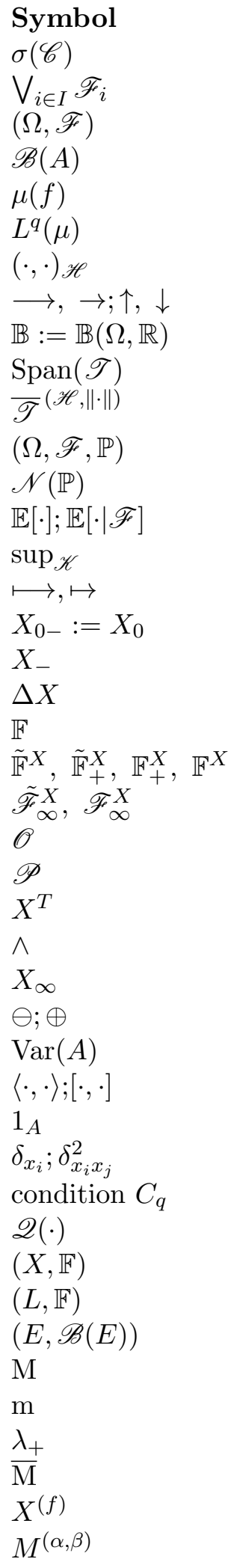

\section{Description}

$\sigma$-algebra generated by $\mathscr{C}$

$\sigma$-algebra generated by $\cup_{i \in I} \mathscr{F}_{i}$

measurable space

Borel $\sigma$-algebra on the topological space $A$

integral of $f$ with respect to the measure $\mu$

$L^{q}$-space with respect to the measure $\mu$

scalar product on the Hilbert space $\mathscr{H}$

convergence; monotone convergence

bounded functions on $\Omega$ with values in $\mathbb{R}$

linear hull of $\mathscr{T}$

closure of $\mathscr{T}$ in the Banach space $(\mathscr{H},\|\cdot\|)$

probability space

null-sets of the probability measure $\mathbb{P}$

expectation; conditional expectation

supremum over $\mathscr{K}$

application or functional

left limit of the initial value of a càdlàg process

left-hand limit process of the càdlàg process $X$

jump process of the càdlàg process $X$

filtration

filtration generated by the process $X, \ldots$

$\sigma$-algebra generated by the process $X, \ldots$

optional $\sigma$-algebra

predictable $\sigma$-algebra

process $X$ stopped at $T$

minimum function

terminal variable of the process $X$

direct difference; direct sum

variation process of the process $A$

point brackets process; covariation process

indicator function of the set $A$

partial derivative; second partial derivative

set of the solutions of a martingale problem

additive process relative to the filtration $\mathbb{F}$

Lévy process relative to the filtration $\mathbb{F}$

measurable space $\left(\mathbb{R}_{+} \times \mathbb{R}, \mathscr{B}\left(\mathbb{R}_{+}\right) \otimes \mathscr{B}(\mathbb{R})\right)$

Poisson random measure

intensity measure

Lebesgue measure on $\left(\mathbb{R}_{+}, \mathscr{B}\left(\mathbb{R}_{+}\right)\right)$

compensated Poisson random measure

compensated-covariation process
Page

2

2

2

2

3

3

3

4

4

5

5 


\section{Acknowledgments}

I would like to express my deepest gratitude to my advisor Hans-Jürgen Engelbert not only because this work would have been not possible without his help, but above all because in this years he taught me with passion and patience the art of being a mathematician.

I thank Yuri Kabanov and Ilya Pavlyukevich because they agreed to spend their time for reading and evaluating my thesis.

I thank Alberto Barchielli because he played a fundamental role in my education and he was always ready to help me every time I asked.

To apply for a PhD position in the ITN was a suggestion of Carlo Sgarra and I thank him for this very good idea.

I acknowledge the secretary of the Department of Stochastics of the faculty of Mathematics and Computer Science of "Friedrich-Schiller-University Jena" Nicole Hickethier for supporting my work with her competence.

In this years I discussed of my work with several people. Among others I thank Monique Jeanblanc, Monique Pontier, Francesco Russo and Martina Zähle.

I thank all my colleagues of the ITN, in particular Andrii Andrusiv, Matteo Bedini, Christine Grün, Elena Issoglio and Holger Metzler. Among my colleagues at "Friedrich-Schiller-University Jena" I specially acknowledge Stefan Blei and Lev Markhasin.

Ringrazio i miei genitori Maria e Antonio e mia sorella Marta per il grande sostegno e affetto che mi hanno dato.

Ich danke Ortrun, das Beste und Schönste was mir in diesen Jahren passiert ist. 



\section{Zusammenfassung}

Im Rahmen von Lévy-Prozessen, untersuchen wir die vorhersagbare Darstellbarkeitseigenschaft, die wir mit PRP abkürzen (von dem englischen Ausdruck "predictable representation property"). Um eine allgemeine Definition der PRP zu geben, verwenden wir die Theorie der stabilen Räume. Sei $L$ ein Lévy-Prozess bezüglich seiner vervollständigten erzeugten Filtration $\mathbb{F}^{L}$ und sei $\nu$ das zugehörige Lévy-Maß. Wir konstruieren Familien von $\mathbb{F}^{L}$-Martingalen, welche die PRP besitzen. Die Martingale, die wir betrachten, werden mittels der stochastischen Integration von deterministischen Funktionen bezüglich des kompensierten Poissonschen zufälligen Sprungmaßes von $L$ erzeugt.

Als Nächstes erklären wir das Obenstehende im Detail. Wir betrachten einen Lévy-Prozess bezüglich seiner vervollständigten erzeugten Filtration $\mathbb{F}^{L}$. Sei $N$ ein lokales Martingal bezüglich $\mathbb{F}^{L}$ und $q=1,2$. Wir definieren die Norm

$$
\|N\|_{\mathscr{H}^{q}}:=\mathbb{E}\left[\sup _{t \geq 0}\left|N_{t}\right|\right]^{\frac{1}{q}}
$$

und bezeichnen den Raum aller $\mathbb{F}^{L}$-adaptierten Martingale $N$, für die $N_{0}=0$ und $\|N\|_{\mathscr{H}^{q}}<+\infty$, mit $\mathscr{H}_{0}^{q}$. Der Raum $\left(\mathscr{H}_{0}^{q},\|\cdot\|_{\mathscr{H}^{q}}\right)$ ist ein Banach-Raum.

Sei $\mathrm{M}$ das Sprungmaß von $L$. Es ist bekannt, dass $M$ ein Poissonsches zufälliges Maß auf dem messbaren Raum $\left(\mathbb{R}_{+} \times \mathbb{R}, \mathscr{B}\left(\mathbb{R}_{+}\right) \otimes \mathscr{B}(\mathbb{R})\right)$ ist. Das Intensitätsmaß von $\mathrm{M}$ ist das Produktmaß $\mathrm{m}:=\lambda_{+} \otimes \nu$, wobei $\lambda_{+}$das Lebesgue-Maß auf der positiven Halblinie $\mathbb{R}_{+}$bezeichnet und $\nu$ das Lévy-Maß von $L$. Wir setzen $\overline{\mathrm{M}}:=\mathrm{M}-\mathrm{m}$ auf dem Ring $\{A$ Borel-Menge : $\mathrm{m}(A)<+\infty\}$ und nennen $\overline{\mathrm{M}}$ das kompensierte Poissonsche zufällige Maß. Dieses ist ein orthogonales Maß und es ist wohlbekannt, wie man diesbezüglich das stochastische Integral deterministischer Funktionen in $L^{2}\left(\lambda_{+} \otimes \nu\right)$ definiert. Für $f \in L^{2}(\nu)$ führen wir den Prozess $X^{(f)}=\left(X_{t}^{(f)}\right)_{t \geq 0}$ mit

$$
X_{t}^{(f)}:=\int_{\mathbb{R}_{+} \times \mathbb{R}} 1_{[0, t]}(s) f(x) \overline{\mathrm{M}}(\mathrm{d} s, \mathrm{~d} x)=\int_{0}^{t} f(x) \overline{\mathrm{M}}(\mathrm{d} s, \mathrm{~d} x), \quad t \geq 0,
$$

ein. Wir werden sehen, dass $X^{(f)}$ ein $\mathbb{F}^{L}$-adaptiertes, lokal quadratisch integrierbares Martingal ist. Mit $\mathrm{W}^{\sigma}$ bezeichnen wir den Gaußschen Anteil des Lévy-Prozesses $L$, 
welcher ein Wiener-Prozess mit $\mathbb{E}\left[\mathrm{W}_{t}^{\sigma 2}\right]=t \sigma^{2}, \sigma^{2} \geq 0, t \geq 0$, ist. Nun betrachten wir ein Funktionensystem $\mathscr{T} \subseteq L^{2}(\nu)$ und führen die Martingalfamilie

$$
\mathscr{X}_{\mathscr{T}}:=\left\{\mathrm{W}^{\sigma}\right\} \cup\left\{X^{(f)}, \quad f \in \mathscr{T}\right\}
$$

ein. Wir beweisen, dass $\mathscr{X}_{\mathscr{T}}$ die $\mathscr{H}^{1}$-PRP besitzt, falls $\mathscr{T}$ total in $L^{2}(\nu)$ ist (d.h. die lineare Hülle dicht in $L^{2}(\nu)$ liegt). Für den Fall $\mathscr{H}_{0}^{2}$ zeigen wir, dass $\mathscr{X}_{\mathscr{T}}$ die $\mathscr{H}^{2}$-PRP genau dann hat, wenn $\mathscr{T}$ total in $L^{2}(\nu)$ liegt. Das bedeutet, dass wir eine Charakterisierung der $\mathscr{H}^{2}$-PRP geben. Die $\mathscr{H}^{2}$-PRP ist besonders interessant, falls $\mathscr{T} \subseteq L^{2}(\nu)$ ein totales System ist, welches aus orthogonalen Funktionen besteht.

Der Hauptteil dieser Dissertation unterteilt sich in fünf Kapitel, denen sich zwei Anhänge anschließen.

In Kapitel 1 tragen wir die benötigten grundlegenden Resultate der Maßtheorie und der stochastischen Analysis zusammen. Insbesondere rufen wir die Definition des stochastischen Integrals bezüglich eines lokalen Martingals in Erinnerung und skizzieren die Theorie stabiler Unterräume. Anschließend geben wir die Definition der $\mathscr{H}^{q}$-PRP.

In Kapitel 2 führen wir einige grundlegende Eigenschaften stochastisch stetiger Prozesse mit unabhängigen Zuwächsen relativ zur einer Filtration ein. Weiterhin definieren wir das stochastische Integral deterministischer Funktionen bezüglich eines Poissonschen zufälligen Maßes und eines kompensierten Poissonschen zufälligen Maßes. Durch diese Art der stochastischen Integration konstruieren wir Lévy-Prozesse.

In Kapitel 3 zeigen wir mit Hilfe eines geeigneten Martingalproblems, dass $\mathscr{X}_{\mathscr{T}}$ die $\mathscr{H}^{1}$-PRP besitzt, falls $\mathscr{T}$ total in $L^{2}(\nu)$ ist. Wir betonen, dass es uns nicht gelungen ist, eine Charakterisierung der $\mathscr{H}^{1}$-PRP zu finden.

In Kapitel 4 wenden wir uns dem Problem der $\mathscr{H}^{2}$-PRP zu und beweisen, dass die Familie $\mathscr{X}_{\mathscr{T}}$ die $\mathscr{H}^{2}$-PRP genau dann besitzt, wenn $\mathscr{T}$ total in $L^{2}(\nu)$ ist. Ein wichtiges Resultat dieses Kapitels betrifft totale Orthogonalsysteme in $L^{2}(\nu)$.

In Kapitel 5 stellen wir Anwendungen der entwickelten Theorie zu konkreten Beispielen vor.

Auf den Hauptteil folgen zwei Anhänge. Anhang A ist eine Ergänzung zu Kapitel 2. In Anhang B diskutieren wir hinreichende Bedingungen, um zu versichern, dass von einer beliebigen Familie $Z:=\left(Z_{\alpha}\right)_{\alpha \in I}$ quadratisch integrierbarer Zufallsvariablen erzeugte Polynome in $L^{2}\left(\Omega, \mathscr{F}^{Z}, \mathbb{P}\right)$ dicht sind, wobei $\mathscr{F}^{Z}$ die von $Z$ erzeugte $\sigma$-Algebra bezeichnet. 
We investigate the predictable representation property (PRP) in the frame of Lévy processes. To give a general definition of the PRP we use the theory of stable subspaces. Let $L$ be a Lévy process relative to its augmented generated filtration $\mathbb{F}^{L}$ and let $\nu$ be its Lévy measure. We construct families of $\mathbb{F}^{L}$-martingales which possess the PRP. The martingales that we consider are obtained via stochastic integration of deterministic functions with respect to the compensated Poisson random measure of the jumps of $L$.

We now explain in more details the above-mentioned. We consider a Lévy process $L$ relative to its completed generated filtration $\mathbb{F}^{L}$. Let $N$ be an $\mathbb{F}^{L}$-adapted local martingale and $q=1,2$. We define the norm

$$
\|N\|_{\mathscr{H}^{q}}:=\mathbb{E}\left[\sup _{t \geq 0}\left|N_{t}\right|\right]^{\frac{1}{q}}
$$

and denote by $\mathscr{H}_{0}^{q}$ the space of all $\mathbb{F}^{L}$-adapted martingales $N$ starting at zero such that $\|N\|_{\mathscr{H}^{q}}<+\infty$. The space $\left(\mathscr{H}_{0}^{q},\|\cdot\|_{\mathscr{H}^{q}}\right)$ is a Banach space.

Let $\mathrm{M}$ be the jump measure of $L$. It is well-known that $\mathrm{M}$ is a Poisson random measure on $\mathbb{R}_{+} \times \mathbb{R}$ with intensity measure $\mathrm{m}:=\lambda_{+} \otimes \nu$, where $\lambda_{+}$is the Lebesgue measure on $\mathbb{R}_{+}$and $\nu$ the Lévy measure of $L$. We put $\overline{\mathrm{M}}:=\mathrm{M}-\mathrm{m}$ on the ring $\{A$ Borel set : $\mathrm{m}(A)<+\infty\}$ and call $\overline{\mathrm{M}}$ the compensated Poisson random measure. The signed random measure $\overline{\mathrm{M}}$ is an orthogonal measure and it is well-understood how to define the integral of functions in $L^{2}\left(\lambda_{+} \otimes \nu\right)$ with respect to $\overline{\mathrm{M}}$. If $f \in L^{2}(\nu)$, we introduce the process $X^{(f)}=\left(X_{t}^{(f)}\right)_{t \geq 0}$ by integrating the function $1_{[0, t]} f$ with respect to $\overline{\mathrm{M}}$, i.e.,

$$
X_{t}^{(f)}:=\int_{\mathbb{R}_{+} \times \mathbb{R}} 1_{[0, t]}(s) f(x) \overline{\mathrm{M}}(\mathrm{d} s, \mathrm{~d} x)=\int_{0}^{t} f(x) \overline{\mathrm{M}}(\mathrm{d} s, \mathrm{~d} x), \quad t \geq 0 .
$$

We shall see that $X^{(f)}$ is a locally square integrable $\mathbb{F}^{L}$-adapted martingale, for every $f \in L^{2}(\nu)$. Let $\mathrm{W}^{\sigma}$ be the Gaussian part of $L$. It is known that $\mathrm{W}^{\sigma}$ is a Wiener process such that $\mathbb{E}\left[\mathrm{W}_{t}^{\sigma 2}\right]=\sigma^{2} t, \sigma^{2} \geq 0, t \geq 0$. For a system $\mathscr{T} \subseteq L^{2}(\nu)$, we introduce the family

$$
\mathscr{X}_{\mathscr{T}}:=\left\{\mathrm{W}^{\sigma}\right\} \cup\left\{X^{(f)}, \quad f \in \mathscr{T}\right\} .
$$


We prove that if $\mathscr{T}$ is total in $L^{2}(\nu)$, then $\mathscr{X}_{\mathscr{T}}$ has the $\mathscr{H}^{1}$-PRP. In case of $\mathscr{H}_{0}^{2}$, we show that $\mathscr{X}_{\mathscr{T}}$ possesses the $\mathscr{H}^{2}$-PRP if and only if $\mathscr{T}$ is total in $L^{2}(\nu)$, that is, in this case we give a characterization of the predictable representation property. The case of the $\mathscr{H}^{2}$-PRP for $\mathscr{X}_{\mathscr{T}}$ is particularly interesting if $\mathscr{T}$ is a total system in $L^{2}(\nu)$ consisting of orthogonal functions.

The main part of the thesis is divided in five chapters to which follow two appendixes.

In Chapter 1 we collect some basic results of measure theory and of stochastic analysis. In particular, we recall the definition of the stochastic integral with respect to a local martingale and we sketch the theory of stable subspaces. Then we give the definition of $\mathscr{H}^{q}$-PRP.

In Chapter 2 we recall some basic properties of stochastically continuous processes with independent increments and of Poisson random measures. Furthermore, we define the stochastic integral of deterministic functions with respect to a Poisson random measure and a compensated Poisson random measure. By means of this stochastic integration we construct Lévy processes.

In Chapter 3, with the help of an appropriate martingale problem, we show that the family $\mathscr{X}_{\mathscr{T}}$ possesses the $\mathscr{H}^{1}$-PRP if $\mathscr{T} \subseteq L^{2}(\nu)$ is total. We stress that we were not able to give a characterization of the $\mathscr{H}^{1}$-PRP.

In Chapter 4 we face the problem of the $\mathscr{H}^{2}$-PRP: We prove that the family $\mathscr{X}_{\mathscr{T}}$ possesses the $\mathscr{H}^{2}$-PRP if and only if $\mathscr{T}$ is total in $L^{2}(\nu)$. One of the most important results of this chapter concerns total orthogonal systems in $L^{2}(\nu)$.

In Chapter 5 we present applications of the developed theory to some concrete examples.

Two appendices conclude the thesis. Appendix A is a complement to Chapter 2. In Appendix B we discuss sufficient conditions to ensure that the family of polynomials generated by an arbitrary family $Z:=\left(Z_{\alpha}\right)_{\alpha \in I}$ of square integrable random variables is dense in $L^{2}\left(\Omega, \mathscr{F}^{Z}, \mathbb{P}\right)$, where $\mathscr{F}^{Z}$ is the $\sigma$-algebra generated by $Z$. 


\section{Description of the Thesis}

The aim of this $\mathrm{PhD}$ thesis is to investigate the predictable representation property $(\mathrm{PRP})$ in the frame of Lévy processes. Let $(\Omega, \mathscr{F}, \mathbb{P})$ be a complete probability space and $\mathbb{F}$ a filtration of $\sigma$-algebras of $\Omega$ satisfying the usual conditions. As a first step, we specify what we mean by PRP. To this aim we need to introduce the stochastic integral with respect to a local martingale and stable subspaces generated by families of local martingales. We refer to Jacod (1979), Chapter II and Chapter IV. Let $M$ be a local martingale. We introduce the norm $\|\cdot\|_{\mathscr{H}^{q}}$ by

$$
\|M\|_{\mathscr{H} q}:=\left(\mathbb{E}\left[\sup _{t \geq 0}|M|^{q}\right]\right)^{\frac{1}{q}}
$$

and the space of $q$-integrable martingales $\mathscr{H}^{q}$ by

$$
\mathscr{H}^{q}:=\left\{M \text { martingale such that }\|M\|_{\mathscr{H}^{q}}<+\infty\right\} .
$$

The class $\mathscr{H}_{0}^{q}$ is the class of the martingales in $\mathscr{H}^{q}$ starting at 0 , while the classes $\mathscr{H}_{\mathrm{loc}}^{q}$ and $\mathscr{H}_{\mathrm{loc}, 0}^{q}$ are introduced from $\mathscr{H}^{q}$ and $\mathscr{H}_{0}^{q}$, respectively, by localization. With a local martingale $M$ we associate the process $[M, M]=\left([M, M]_{t}\right)_{t \geq 0}$ defined by

$$
[M, M]_{t}:=\left\langle M^{c}, M^{c}\right\rangle_{t}+\sum_{0<s \leq t}\left(\Delta M_{s}\right)^{2}, \quad t \geq 0 .
$$

This is a process of finite variation and it is well-understood how to define the integral with respect to it for nonnegative measurable processes. We consider a local martingale $M$ and fix $q \in[1,+\infty)$. For a measurable process $H$ we define the norm $\|\cdot\|_{\mathrm{L}^{q}(M)}$ by

$$
\|H\|_{\mathrm{L}^{q}(M)}:=\left(\mathbb{E}\left[\left(\int_{0}^{\infty} H_{s}^{2} \mathrm{~d}[M, M]_{s}\right)^{\frac{q}{2}}\right]\right)^{\frac{1}{q}}
$$

and then the space of processes

$$
\mathrm{L}^{q}(M):=\left\{H \text { predictable }:\|H\|_{\mathrm{L}^{q}(M)}<+\infty\right\} .
$$


The class $\mathrm{L}_{\text {loc }}^{q}(M)$ is obtained from $\mathrm{L}^{q}(M)$ in the following way: We say that a predictable process $H$ belongs to $\mathrm{L}_{\text {loc }}^{q}(M)$ if there exists a sequence $\left(T_{n}\right)_{n \geq 1}$ of stopping times such that $T_{n} \uparrow+\infty$ and that the predictable process $H 1_{\left[0, T_{n}\right]}$ belongs to $\mathrm{L}^{q}(M), n \geq 1$. Obviously, for every $p \leq q$, the inclusion $\mathrm{L}_{\text {loc }}^{q}(M) \subseteq \mathrm{L}_{\text {loc }}^{p}(M)$ holds. The stochastic integral with respect to $M$ for a predictable process $H$ belonging $\mathrm{L}_{\text {loc }}^{1}(M)$ is defined as the unique element of $\mathscr{H}_{\text {loc }, 0}^{1}$, say $X$, such that

$$
[X, N]=\int H \mathrm{~d}[M, N], \quad N \in \mathscr{H}_{\mathrm{loc}, 0}^{1} .
$$

Moreover, $\mathrm{L}_{\text {loc }}^{1}(M)$ is the largest class of predictable processes such that the stochastic integral with respect to $M$ is again a local martingale. We denote the element $X$ as above by

$$
X:=H \cdot M \text {. }
$$

We notice that the stochastic integral with respect to $M$ of a predictable process $H$ belongs to $\mathscr{H}_{0}^{q}$ (respectively $\mathscr{H}_{\text {loc }, 0}^{q}$ ) if and only if $H$ belongs to $\mathrm{L}^{q}(M)$ (respectively $\left.\mathrm{L}_{\text {loc }}^{q}(M)\right)$. Now we introduce stable subspaces. This notion is specially important for our purposes because it allows to give a general definition of the PRP. We consider $q \in[1,+\infty)$ and the space of $q$-integrable martingales starting at $0, \mathscr{H}_{0}^{q}$. We say that a linear subspace $\mathscr{H} \subseteq \mathscr{H}_{0}^{q}$ is a stable subspace of $\mathscr{H}_{0}^{q}$ if it is closed in $\left(\mathscr{H}_{0}^{q},\|\cdot\|_{\mathscr{H}^{q}}\right)$ and it is stable under stopping, i.e., such that if $M \in \mathscr{H}$, then $M^{T} \in \mathscr{H}$ for every stopping time $T$. The property of the stability under stopping is a particular operation of stochastic integration. Indeed, we have $M^{T}=1_{[0, T]} \cdot M$ and the process $1_{[0, T]}$ is predictable and belongs to $\mathrm{L}^{q}(M)$. More generally, a stable subspace is characterized as it follows. A linear space $\mathscr{H} \subseteq \mathscr{H}_{0}^{q}$ which is closed in $\left(\mathscr{H}_{0}^{q},\|\cdot\|_{\mathscr{C}^{q}}\right)$ is a stable subspace of $\mathscr{H}_{0}^{q}$ if and only if it is stable under stochastic integration, i.e., for every $M \in \mathscr{H}$ and $H \in \mathrm{L}^{q}(M)$ the stochastic integral $H \cdot M$ belongs to $\mathscr{H}$. The notion of stable subspace raises the definition of stable subspace generated by a family of local martingales. Let $\mathscr{X}$ be a family of local martingales starting at zero and $q \geq 1$. We know that the set

$$
\mathscr{X}_{q}:=\left\{H \cdot M, H \in \mathrm{L}^{q}(M), M \in \mathscr{X}\right\}
$$

is contained in $\mathscr{H}_{0}^{q}$. We define the stable subspace generated by $\mathscr{X}$ in $\mathscr{H}_{0}^{q}$ as the smallest linear subspace of $\mathscr{H}_{0}^{q}$ which is closed in $\left(\mathscr{H}_{0}^{q},\|\cdot\|_{\mathscr{H}^{q}}\right)$ and contains $\mathscr{X}_{q}$. We denote it by $\mathscr{L}^{q}(\mathscr{X})$. Now we can give the general notion of the PRP. We say that the family of local martingales $\mathscr{X}$ has the $\mathscr{H}^{q}$-PRP relative to $\mathbb{F}$ if the stable subspace generated by $\mathscr{X}$ in $\mathscr{H}_{0}^{q}$ equals $\mathscr{H}_{0}^{q}$ itself, i.e., if

$$
\mathscr{L}^{q}(\mathscr{X})=\mathscr{H}_{0}^{q} .
$$

Historically, the PRP was first studied for a single local martingale or for finite families of local martingale and not in full generality. In case of a single martingale we can reformulate the PRP as it follows. An $\mathbb{F}$-local martingale $M$ has the PRP relative to $\mathbb{F}$ if for any $\mathbb{F}$-adapted local martingale $N$ there exists a predictable process $H^{N} \in \mathrm{L}_{\text {loc }}(N)$ such that

$$
N=N_{0}+H^{N} \cdot M
$$


A seminal paper for the study of the PRP is certainly Itô (1951), where the multiple stochastic integral for the Wiener process was defined. In Kunita \& Watanabe (1967) and Clark (1970) it was proven that if $M$ is a Wiener process on the probability space $\left(\Omega, \mathscr{F}_{\infty}^{M}, \mathbb{P}\right)$, where $\mathscr{F}_{\infty}^{M}$ is the completion of the $\sigma$-algebra $\sigma\left(M_{t}, t \geq 0\right)$, and $\mathbb{F}^{M}$ is the smallest filtration satisfying the usual conditions and containing the filtration generated by $M$, then $M$ possesses the PRP relative to $\mathbb{F}^{M}$. In Kabanov (1973) and Kabanov (1974) a similar result was obtained for the compensated Poisson process. However, the Wiener process and the compensated Poisson process are not the unique martingales possessing the PRP relative to the smallest filtration satisfying the usual conditions and containing their generated filtration. In a series of papers, amongst others Boel, Varaiya \& Wong (1975), Chou \& Meyer (1975), Davis (1976), Elliott (1976), similar representation results were obtained for martingales adapted to the filtration generated by certain kind of jump processes. In Engelbert \& Hess (1980) and Engelbert \& Hess (1981) conditions of probabilistic type were given for certain continuous local martingales to have the classical PRP with respect to their natural augmented filtration. On the other side, it is well-known that a Lévy process $L$ which is a martingale possesses the PRP relative to $\mathbb{F}^{L}$, i.e., $\mathscr{L}^{1}(L)=\mathscr{H}_{0}^{1}\left(\mathbb{F}^{L}\right)$, where $\mathbb{F}^{L}$ is the smallest filtration satisfying the usual conditions and containing the filtration generated by $L$, if and only if it is a Wiener process or a compensated Poisson process. It is clear then, that to obtain new interesting results for Lévy processes, we need to consider the general formulation of the PRP. Let $L$ be a Lévy process relative to $\mathbb{F}^{L}$. With this work we answer to the following question: Can we construct a family $\mathscr{X}$ of $\mathbb{F}^{L}$-local martingales possessing the PRP relative to $\mathbb{F}^{L}$ ? Obviously, if $\mathscr{X}$ is the family of all the $\mathbb{F}^{L}$-local martingales, it possesses the PRP relative to $\mathbb{F}^{L}$, but this case is not of interest and we would like to construct the family $\mathscr{X}$ in such a way to be "as small as possible".

To verify that a family $\mathscr{X}$ of local martingales possesses the $\mathscr{H}^{q}$-PRP, there are two possible strategies. If $q=1$, we can formulate a certain martingale problem for the family $\mathscr{X}$. If the set of the probability measures which are solutions of this martingale problem is a singleton, then $\mathscr{X}$ has the $\mathscr{H}^{1}$-PRP. This result was shown in Jacod \& Yor (1977). The approach by means of the martingale problem is typical of the $\mathscr{H}^{1}$-PRP and it seems that it cannot be applied in general to prove the $\mathscr{H}^{q}$-PRP with $q>1$. The other possible way is, for a fixed $q \geq 1$, to look at the family of the martingales belonging to $\mathscr{H}_{0}^{p}$ which are orthogonal to the family $\mathscr{X}$, where $p$ is the conjugate exponent of $q$, that is, $p \in(1,+\infty]$ such that $\frac{1}{p}+\frac{1}{q}=1$ (we recall that two local martingales $M$ and $N$ are orthogonal if $M N$ is again a local martingale). If $\mathscr{X}$ is contained in $\mathscr{H}_{\text {loc }, 0}^{q}$, then it has the $\mathscr{H}^{q}$-PRP if and only if all the martingales in $\mathscr{H}_{0}^{p}$ which are orthogonal to $\mathscr{X}$ are evanescent. This result is shown in Jacod (1979), Chapter IV.

Let $L$ be a Lévy process. We apply the theory sketched above only to study the $\mathscr{H}^{1}$-PRP and the $\mathscr{H}^{2}$-PRP of certain families of $\mathbb{F}^{L}$-martingales which we now describe.

By a Lévy process $L$ relative to a filtration $\mathbb{F}$ satisfying the usual condition, we mean a stochastically continuous and càdlàg process $L$ starting at zero such that for every $0 \leq s \leq t$, the random variable $L_{t}-L_{s}$ is distributed as $L_{t-s}$ and is independent of $\mathscr{F}_{s}$. We consider only one-dimensional Lévy processes, that is, with 
values in the measurable space $(\mathbb{R}, \mathscr{B}(\mathbb{R}))$. The jump measure $\mathrm{M}$ of $L$ is defined by

$$
\mathrm{M}(\omega, A):=\sum_{s \geq 0} 1_{\left\{\Delta L_{s}(\omega) \neq 0\right\}} 1_{A}\left(s, \Delta L_{s}(\omega)\right), \quad A \in\left(\mathbb{R}_{+} \times \mathbb{R}, \mathscr{B}\left(\mathbb{R}_{+}\right) \otimes \mathscr{B}(\mathbb{R})\right),
$$

and it is an integer-valued random measure $\mathrm{M}$. Furthermore, $\mathrm{M}$ is a Poisson random measure on the measurable space $\left(\mathbb{R}_{+} \times \mathbb{R}, \mathscr{B}\left(\mathbb{R}_{+}\right) \otimes \mathscr{B}(\mathbb{R})\right)$ relative to the filtration $\mathbb{F}$. The definition of Poisson random measures relative to a filtration is given in Jacod \& Shiryaev (2000), §II.1. The intensity measure of M is the product measure $\lambda_{+} \otimes \nu$, where $\lambda_{+}$is the Lebesgue measure on the positive half line $\mathbb{R}_{+}$, and $\nu$ is a $\sigma$-finite measure on $(\mathbb{R}, \mathscr{B}(\mathbb{R}))$ such that $\nu(\{0\})=0$ and the function $\min \left\{x^{2}, 1\right\}$ is integrable with respect to $\nu$. The measure $\nu$ is known in the literature as the Lévy measure of the Lévy process $L$. Let $\mathscr{E}$ be the ring of the $\mathscr{B}\left(\mathbb{R}_{+}\right) \otimes \mathscr{B}(\mathbb{R})$-measurable sets $A$ such that $\left(\lambda_{+} \otimes \nu\right)(A)$ is finite. On $\mathscr{E}$ we define the signed measure $\overline{\mathrm{M}}$ by

$$
\overline{\mathrm{M}}:=\mathrm{M}-\lambda_{+} \otimes \nu .
$$

We call $\overline{\mathrm{M}}$ the compensated Poisson random measure of $L$ : It is an orthogonal measure and it is well-understood how to introduce the stochastic integral of deterministic functions with respect to it (cf. Gihman \& Skorohod (1974), §IV.4). If $f$ belongs to $L^{2}\left(\lambda_{+} \otimes \nu\right)$, the stochastic integral of $f$ with respect to $\bar{M}$, which we denote by $\overline{\mathrm{M}}(f)$, is a square integrable random variable. Moreover, the stochastic integral with respect to $\overline{\mathrm{M}}$ is a (continuous) isometric mapping on $L^{2}\left(\lambda_{+} \otimes \nu\right)$ into $L^{2}(\mathbb{P})$. For $f \in L^{2}(\nu)$ we introduce the process $X^{(f)}=\left(X_{t}^{(f)}\right)_{t \geq 0}$ by

$$
X_{t}^{(f)}:=\overline{\mathrm{M}}\left(1_{[0, t]} f\right), \quad t \geq 0 .
$$

The process $X^{(f)}$ is an $\mathbb{F}$-martingale in $\mathscr{H}_{\mathrm{loc}, 0}^{2}$. Moreover, it is a Lévy process relative to $\mathbb{F}$. Let $\mathrm{W}^{\sigma}$ be the Gaussian part of the Lévy process $L$. The process $\mathrm{W}^{\sigma}$ is a Wiener process relative to $\mathbb{F}$ with variance function $\sigma^{2}(t):=\sigma^{2} t$, where $\sigma^{2} \geq 0$. Let us define the family $\mathscr{X}$ by

$$
\mathscr{X}:=\left\{\mathrm{W}^{\sigma}\right\} \cup\left\{X^{(f)}, f \in L^{2}(\nu)\right\} .
$$

This is a family that we consider to obtain results on the PRP. More precisely, if we define the $\sigma$-algebra $\mathscr{F}_{\infty}^{L}$ as the $\mathbb{P}$-completion of the $\sigma$-algebra $\sigma\left(L_{t}, t \geq 0\right)$ and we restrict our attention to the probability space $\left(\Omega, \mathscr{F}_{\infty}^{L}, \mathbb{P}\right)$, we shall show that the family $\mathscr{X}$ has the $\mathscr{H}^{2}$-PRP with respect to the filtration $\mathbb{F}^{L}$. The $\mathscr{H}^{2}$-PRP implies that $\mathscr{X}$ possesses also the $\mathscr{H}^{1}$-PRP. However, we prove that $\mathscr{X}$ possesses the $\mathscr{H}^{1}$-PRP independently of the $\mathscr{H}^{2}$-PRP as an elegant application of the theory developed in Jacod \& Yor (1977). The simplest way to prove that the family $\mathscr{X}$ introduced in (2) has the $\mathscr{H}^{2}$-PRP relative to $\mathbb{F}^{L}$ is to look at the problem from a more general point of view. We consider a general family $\mathscr{Z}$ of martingales in $\mathscr{H}_{\text {loc }, 0}^{2}$ on the probability space $\left(\Omega, \mathscr{F}_{\infty}^{\mathscr{Z}}, \mathbb{P}\right)$ with respect to the filtration $\mathbb{F}^{\mathscr{Z}}$, where $\mathscr{F}_{\infty}^{\mathscr{Z}}$ is the $\mathbb{P}$-completion of the $\sigma$-algebra $\sigma\left(Z_{t}, t \geq 0, Z \in \mathscr{Z}\right)$ and $\mathbb{F}^{\mathscr{Z}}$ is the smallest filtration satisfying the usual conditions and containing the filtration generated by $\mathscr{Z}$. We give sufficient conditions on $\mathscr{Z}$ to ensure that $\mathscr{L}^{2}(\mathscr{Z})=\mathscr{H}_{0}^{2}$. We obtain as a consequence the $\mathscr{H}^{2}$-PRP for the family $\mathscr{X}$ introduced in (2). Although this 
result is theoretically interesting, the family $\mathscr{X}$ is too large for applications. In particular, it is always uncountable. So the question is now how far the family $\mathscr{X}$ can be diminished without loosing the $\mathscr{H}^{2}$-PRP. In other words, for a system $\mathscr{T} \subseteq L^{2}(\nu)$, we introduce the family $\mathscr{X}_{\mathscr{T}}$ by

$$
\mathscr{X}_{\mathscr{T}}:=\left\{\mathrm{W}^{\sigma}\right\} \cup\left\{X^{(f)}, f \in \mathscr{T}\right\} .
$$

We look for necessary and sufficient conditions on the system $\mathscr{T}$ for the family $\mathscr{X}_{\mathscr{T}}$ to possess the $\mathscr{H}^{2}$-PRP relative to $\mathbb{F}^{L}$. We obtain that the family $\mathscr{X}_{\mathscr{T}}$ possesses the $\mathscr{H}^{2}$-PRP if and only if the system $\mathscr{T}$ is total (i.e., its linear hull is dense) in $L^{2}(\nu)$. The space $L^{2}(\nu)$ is a separable Hilbert space so there always exists a countable system $\mathscr{T} \subseteq L^{2}(\nu)$ which is total. Hence it is always possible to choose a countable subfamily of $\mathscr{X}$ with the $\mathscr{H}^{2}$-PRP. If the system $\mathscr{T}$ consists of orthogonal functions, then the associated family $\mathscr{X}_{\mathscr{T}}$ is an orthogonal family of martingales and if $\mathscr{T}$ is moreover total in $L^{2}(\nu)$, then $\mathscr{X}_{\mathscr{T}}$ is a family of orthogonal martingales with the $\mathscr{H}^{2}$-PRP. In this special case the structure of the stable subspace generated by $\mathscr{X}_{\mathscr{T}}$ in $\mathscr{H}_{0}^{2}$ becomes particularly simple and any martingale $M \in \mathscr{H}_{0}^{2}$ can be written as an infinite sum (converging in $\left.\left(\mathscr{H}^{2},\|\cdot\|_{\mathscr{H}^{2}}\right)\right)$ of stochastic integrals with respect to martingales in $\mathscr{X}_{\mathscr{T}}$, that is, we have

$$
M=\phi \cdot \mathrm{W}^{\sigma}+\sum_{n=1}^{\infty} H^{n} \cdot X^{\left(f_{n}\right)}
$$

where $\phi \in \mathrm{L}^{2}\left(\mathrm{~W}^{\sigma}\right), H^{n} \in \mathrm{L}^{2}\left(X^{\left(f_{n}\right)}\right)$ and $f_{n} \in \mathscr{T}$, for every $n \geq 1$. We also show that the integrands in (4) are uniquely determined by the martingale $M$.

In Nualart \& Schoutens (2000) it was proven that the family of orthogonalized Teugels martingales possesses the $\mathscr{H}^{2}$-PRP with respect to the filtration $\mathbb{F}^{L}$ under rather strong assumptions on the Lévy measure. We are able to obtain the results of Nualart \& Schoutens (2000) as an application of the theory that we developed. We can also find a large number of examples for which the Teugels martingales cannot be introduced and nevertheless we can construct interesting families of orthogonal martingales with the $\mathscr{H}^{2}$-PRP.

\section{The Plan of the Thesis}

The main part of the thesis is divided in five chapters which are followed by two appendixes.

In Chapter 1 we collect some basic results of measure theory and of stochastic analysis. In particular, we recall the definition of the stochastic integral with respect to a local martingale and we sketch the theory of stable subspaces. Then we give the definition of $\mathscr{H}^{q}$-PRP and we discuss some sufficient conditions to ensure that a family $\mathscr{X}$ of local martingales possesses the $\mathscr{H}^{q}$-PRP for $q \in[1,+\infty)$. For this part the main references are Meyer (1966), Dellacherie (1972), Lepingle (1977), Jacod \& Yor (1977), Jacod (1979), Brezis (1983), He, Wang \& Yan (1992), Jacod \& Shiryaev (2000), Bauer (2001) and Protter (2005).

In the first part of Chapter 2 we recall some basic properties of stochastically continuous processes with independent increments relative to a filtration satisfying 
the usual conditions. We also formulate a nice characterization of the independence for families of such processes. The proof of this result require some preparation and we postpone it to Appendix A. The second part of the chapter is devoted to random measures and to Poisson random measures relative to a filtration. We do not consider general Poisson random measure but only homogeneous Poisson random measures associated with the jumps of càdlàg processes. This restriction avoids technical difficulties and we refer to Jacod \& Shiryaev (2000) for a complete treatment. Furthermore, we define the stochastic integral of deterministic functions with respect to a Poisson random measure and a compensated Poisson random measure. For these topics we refer to Gihman \& Skorohod (1974) and Kallenberg (1997). We also explain how to construct Lévy processes by means of integration of deterministic functions with respect to a Poisson random measure. The last part of Chapter 2 is devoted to some general aspects of Lévy processes, like the Itô-Lévy decomposition, the Lévy-Kintchine decomposition and the existence of moments. For this part we refer to He, Wang \& Yan (1992), Kallenberg (1997) and Sato (1999).

In Chapter 3, as an application of Jacod \& Yor (1977), we show that the family $\mathscr{X}$ introduced in (2) possesses the $\mathscr{H}^{1}$-PRP relative to the filtration $\mathbb{F}^{L}$ : We formulate a suitable martingale problem for $\mathscr{X}$ and we show that its solution is unique. Then we prove that the family $\mathscr{X}_{\mathscr{T}}$ defined by (3) possesses the $\mathscr{H}^{1}$-PRP if $\mathscr{T}$ is total in $L^{2}(\nu)$. We stress that in this case we were only able to obtain that the totality of $\mathscr{T}$ in $L^{2}(\nu)$ is a sufficient condition for the family $\mathscr{X}_{\mathscr{T}}$ to have the $\mathscr{H}^{1}$-PRP with respect to $\mathbb{F}^{L}$ and not that this is also necessary. We conclude Chapter 3 discussing some open problems related the $\mathscr{H}^{1}$-PRP.

In Chapter 4 we face the problem of the $\mathscr{H}^{2}$-PRP and we prove that the family $\mathscr{X}_{\mathscr{T}}$ defined as in (3) possesses the $\mathscr{H}^{2}$-PRP if and only if $\mathscr{T}$ is total in $L^{2}(\nu)$. Probably, the most important result of this chapter concerns total orthogonal systems $\mathscr{T}$ in $L^{2}(\nu)$.

In Chapter 5 we present some interesting examples of countable families of orthogonal martingales with the $\mathscr{H}^{2}$-PRP (and hence with the $\mathscr{H}^{1}$-RPP) relative to $\mathbb{F}^{L}$, as the family obtained by the orthogonalization of the Teugels martingales. However, we are able to consider also more general cases of Lévy process for which the Teugels martingales cannot be defined, as, for example, the case in which $L$ is a Cauchy process. Notice that thanks to the results that we obtained, we achieved a large freedom to construct families of martingales with the $\mathscr{H}^{2}$-PRP.

The thesis is concluded by two appendices. In Appendix A we give the proof of the characterization of the independence for stochastically continuous semimartingale with independent increments, of the Itô-Lévy decomposition and of the LévyKintchine decomposition. In Appendix B we discuss sufficient conditions to ensure that the family of polynomials generated by an arbitrary family $Z:=\left(Z_{\alpha}\right)_{\alpha \in I}$ of square integrable random variables is dense in $L^{2}\left(\Omega, \mathscr{F}^{Z}, \mathbb{P}\right)$, where $\mathscr{F}^{Z}$ is the completed $\sigma$-algebra generated by the family $Z$. 


\section{CHAPTER 1}

\section{Preliminaries}

In this chapter we collect some of the results of measure theory and of stochastic analysis needed for this work. $\S 1.1$ is devoted to basics of measure theory, while $\S 1.2$ to generalities on stochastic processes. The most important parts are $\S 1.3$ and $\S 1.4$ which deal with stochastic integration and stable subspaces of martingales, respectively. In particular, in $\$ 1.4$ the definition of the predictable representation property for an arbitrary family of local martingales is given. Most of the results included in this chapter are stated without proof and we refer, among others, to Meyer (1966), Dellacherie (1972), Jacod \& Yor (1977), Lepingle (1977), Jacod (1979), He, Wang \& Yan (1992), Jacod \& Shiryaev (2000), Bauer (2001) and Brezis (1983) for a more detailed treatment.

\subsection{Measure Theory}

In this section we present some well-known facts of measure theory, like the abstract integration, the uniqueness theorem for measures, the theorem of Lebesgue on dominated convergence, the theorem of $\mathrm{B}$. Levi on monotone convergence and some formulations of monotone class theorems. We also briefly discuss the totality of systems of functions in $L^{q}$-spaces.

\subsubsection{Measurability and Integration}

We consider an arbitrary nonempty set $\Omega$. If $A \subseteq \Omega$ we denote by $A^{c}$ the complement of $A$ in $\Omega$. Let $\left(A_{n}\right)_{n \geq 1}$ be a sequence of subsets of $\Omega$ and $A \subseteq \Omega$. If $A_{n} \subseteq A_{n+1}$, $n \geq 1$, and $A=\cup_{n=1}^{\infty} A_{n}$, we write $A_{n} \uparrow A$. If $A_{n+1} \subseteq A_{n}, n \geq 1$, and $A=\cap_{n=1}^{\infty} A_{n}$, we write $A_{n} \downarrow A$.

A system $\mathscr{R}$ of subsets of $\Omega$ is called a semiring of subsets of $\Omega$ if it possesses the following properties: The empty set belongs to $\mathscr{R}$; if $A$ and $B$ belong to $\mathscr{R}$, then their intersection $A \cap B$ does; if $A$ and $B$ belong to $\mathscr{R}$ and $A \subseteq B$, then the set-difference $B \backslash A$ can be written as finite union of pairwise disjoint elements of 
$\mathscr{R}$. A system $\mathscr{R}$ of subsets of $\Omega$ with the following properties is called a ring: The empty set belongs to $\mathscr{R}$; if $A$ and $B$ belong to $\mathscr{R}$, then their union $A \cup B$ and their set-difference $A \backslash B$ do. Notice that a ring contains also the intersection of two of its elements because $A \cap B=A \backslash(A \backslash B)$. Obviously a ring is also a semiring.

1.1.1 Definition. A system $\mathscr{F}$ of subsets of $\Omega$ is called an algebra (in $\Omega$ ) if it has the following properties:

(i) $\Omega \in \mathscr{F}$;

(ii) if $A \in \mathscr{F}$, then $A^{c} \in \mathscr{F}$;

(iii) if $A, B \in \mathscr{F}$, then $A \cup B \in \mathscr{F}$.

If (iii) is replaced by

(iii') if $\left(A_{n}\right)_{n \in \mathbb{N}} \subseteq \mathscr{F}$, then $\cup_{n \in \mathbb{N}} A_{n} \in \mathscr{F}$, then $\mathscr{F}$ is denominated a $\sigma$-algebra (in $\Omega$ ).

We notice that an algebra is a ring that in addition contains $\Omega$. If $\mathscr{C} \subseteq \Omega$ is a system of sets, the $\sigma$-algebra generated by $\mathscr{C}$ is denoted by $\sigma(\mathscr{C})$ and is defined as the smallest $\sigma$-algebra containing $\mathscr{C}$. If $\mathscr{C} \subseteq \mathscr{F}$ is such that $\sigma(\mathscr{C})=\mathscr{F}$ we say that $\mathscr{C}$ generates $\mathscr{F}$ and call it a generator of $\mathscr{F}$. If $\mathscr{C}$ is a generator of $\mathscr{F}$ which is stable under intersection of two sets, we call it an $\cap$-stable generator. If the $\sigma$-algebra $\mathscr{F}$ can be generated by a countable system $\mathscr{C}$, we say that it is a separable $\sigma$-algebra. Let $(\mathscr{C})_{i \in I}$ be a family of systems of subsets in $\Omega$, where $I$ is an arbitrary set of indexes. By $\bigvee_{i \in I} \mathscr{C}_{i}$ we denote the $\sigma$-algebra generated by the union of all the $\mathscr{C}_{i} \mathrm{~s}$, that is, $\bigvee_{i \in I} \mathscr{C}_{i}:=\sigma\left(\bigcup_{i \in I} \mathscr{C}_{i}\right)$. Let $\Omega$ be a topological space. We denote by $\mathscr{B}(\Omega)$ the Borel $\sigma$-algebra on $\Omega$, i.e., the $\sigma$-algebra generated in $\Omega$ by the open sets in the topology of $\Omega$. If, for example, $\Omega=\mathbb{R}$, then $\mathscr{B}(\mathbb{R})$ is separable.

For any $\sigma$-algebra $\mathscr{F}$ of $\Omega$, we call the couple $(\Omega, \mathscr{F})$ a measurable space and we say that the subsets of $\Omega$ which belong to $\mathscr{F}$ are $\mathscr{F}$-measurable or simply measurable. We consider two measurable spaces $(\Omega, \mathscr{F})$ and $\left(\Omega^{\prime}, \mathscr{F}^{\prime}\right)$ and a function $f$ from $\Omega$ into $\Omega^{\prime}$. We say that $f$ is $\left(\mathscr{F}, \mathscr{F}^{\prime}\right)$-measurable or simply measurable, if for any $A^{\prime} \in \mathscr{F}^{\prime}$ the set $f^{-1}\left(A^{\prime}\right):=\left\{a \in \Omega: f(a) \in A^{\prime}\right\}$ is $\mathscr{F}$-measurable. We call the set $f^{-1}\left(A^{\prime}\right)$ the inverse image of $A^{\prime}$ by $f$. If $f$ is a function on $(\Omega, \mathscr{F})$ into $\left(\Omega^{\prime}, \mathscr{F}^{\prime}\right)$ the system of sets $f^{-1}\left(\mathscr{F}^{\prime}\right):=\left\{f^{-1}\left(A^{\prime}\right): A^{\prime} \in \mathscr{F}^{\prime}\right\}$ is a $\sigma$-algebra in $\Omega$. Let $\left\{\left(\Omega_{i}, \mathscr{F}_{i}\right): i \in I\right\}$ be a family of measurable spaces and $\left\{f_{i}: i \in I\right\}$ be a family of functions on $\Omega$ such that $f_{i}$ takes values in $\Omega_{i}$, for every $i \in I$. The $\sigma$-algebra in $\Omega$ generated by $\bigcup_{i \in I} f_{i}^{-1}\left(\mathscr{F}_{i}\right)$ is the smallest $\sigma$-algebra $\mathscr{F}^{\prime}$ with respect to which every $f_{i}$ is $\left(\mathscr{F}^{\prime}, \mathscr{F}_{i}\right)$-measurable. We designate this $\sigma$-algebra by $\sigma\left(f_{i}: i \in I\right)$, that is, $\sigma\left(f_{i}: i \in I\right):=\bigvee_{i \in I} f_{i}^{-1}\left(\mathscr{F}_{i}\right)$ and we call it the $\sigma$-algebra generated by $\left\{f_{i}: i \in I\right\}$.

Let $(\Omega, \mathscr{F})$ be a measurable space. A set-function $\mu$ on $\mathscr{F}$ into $[0,+\infty]$ such that $\mu(\emptyset)=0$ and that $\mu\left(\bigcup_{n \in \mathbb{N}} A_{n}\right)=\sum_{n=1}^{\infty} \mu\left(A_{n}\right)$, for any sequence $\left(A_{n}\right)_{n \in \mathbb{N}} \subseteq \mathscr{F}$ of pairwise-disjoint sets, is called a measure on $(\Omega, \mathscr{F})$. If $\mu$ takes values in $[-\infty,+\infty]$, then it is called a signed measure. If $\mu$ is a measure on $(\Omega, \mathscr{F})$, we say that $(\Omega, \mathscr{F}, \mu)$ is a measure space. A measure $\mu$ such that $\mu(\Omega)<+\infty$ is called a finite measure. If there exists an increasing sequence $\left(A_{n}\right)_{n \in \mathbb{N}} \subseteq \mathscr{F}$ such that $\mu\left(A_{n}\right)<+\infty$ for every $n \in \mathbb{N}$ and $\bigcup_{n \in \mathbb{N}} A_{n}=\Omega$, then the measure $\mu$ is called $\sigma$-finite. If $\Omega$ is a Hausdorff space with $\sigma$-algebra $\mathscr{B}(\Omega)$, we say that $\mu$ is locally finite if every point of $\Omega$ has an open neighborhood of finite measure $\mu$. The following result holds for 
$\sigma$-finite measures and it is well-known in the literature as uniqueness theorem (cf., e.g., Bauer (2001), Theorem I.5.4).

1.1.2 Theorem (Uniqueness theorem). Let $(\Omega, \mathscr{F})$ be a measurable space, $\mathscr{C} \subseteq \mathscr{F}$ an $\cap$-stable generator of $\mathscr{F}$ and $\left(A_{n}\right)_{n \in \mathbb{N}} \subseteq \mathscr{C}$ satisfying the property $\bigcup_{n \in \mathbb{N}} A_{n}=\Omega$. We suppose that $\mu_{1}$ and $\mu_{2}$ are $\sigma$-finite measures on $\mathscr{F}$ such that

(i) $\mu_{1}(A)=\mu_{2}(A)$, for every $A \in \mathscr{C}$;

(ii) $\mu_{1}\left(A_{n}\right)=\mu_{2}\left(A_{n}\right)<+\infty$, for every $n \in \mathbb{N}$.

Then $\mu_{1}$ and $\mu_{2}$ are identical on $\mathscr{F}$.

For a measure $\mu$ on the measurable space $(\Omega, \mathscr{F})$, it is well understood how to define the integral of a measurable function with values in $(\mathbb{R}, \mathscr{B}(\mathbb{R}))$. We introduce the notation

$$
\mu(f):=\int_{\Omega} f \mathrm{~d} \mu:=\int_{\Omega} f(x) \mu(\mathrm{d} x)
$$

if the integral on the right-hand side exists. In particular, $\mu(f)$ is well defined if $f$ is nonnegative. We say that a measurable function $f$ of arbitrary sign is $\mu$-integrable or simply integrable if $\mu(|f|)<+\infty$. We do not go into details and we refer to Bauer (2001), Chapter II. By functions, if not otherwise specified, we mean functions with values in $(\mathbb{R}, \mathscr{B}(\mathbb{R}))$, that is numerical functions. Let $f$ be a measurable function. By $\|f\|_{q}$ we denote the following norm

$$
\|f\|_{q}:= \begin{cases}\mu\left(|f|^{q}\right)^{\frac{1}{q}}, & q \in[1,+\infty), \\ {\operatorname{ess} \sup _{x \in \Omega}|f(x)|,} q=+\infty\end{cases}
$$

and we put

$$
L^{q}(\mu):=\left\{f \text { measurable }:\|f\|_{q}<+\infty\right\}, \quad q \in[1,+\infty] .
$$

We recall that $f \in L^{q}(\mu)$ is uniquely determined up to equivalence classes $\mu$-a.e. Sometimes, we write $L^{q}(\Omega, \mathscr{F}, \mu)$ to stress the measure space and $\|\cdot\|_{L^{q}(\mu)}$ to stress the space $L^{q}(\mu)$. The space $\left(L^{q}(\mu),\|\cdot\|_{q}\right)$ is a Banach space. The space $L^{2}(\mu)$ is especially important because it is a Hilbert space with respect to the scalar product $(f, g)_{L^{2}(\mu)}:=\mu(f g)$. If $f, g \in L^{2}(\nu)$ are such that $(f, g)_{L^{2}(\mu)}=0$, we say that they are orthogonal (in $L^{2}(\mu)$ ) and denote it by $f \perp g$. If $\mathscr{G}$ is a subset of functions in $L^{2}(\nu)$ and $f \in L^{2}(\nu)$ is such that $f \perp g$ for every $g \in \mathscr{G}$, we say that $f$ is orthogonal (in $L^{2}(\mu)$ ) to $\mathscr{G}$ and we denote it by $f \perp \mathscr{G}$. For a finite measure $\mu$, the inclusions $L^{q}(\mu) \subseteq L^{p}(\mu), 1 \leq p \leq q$, hold. In particular, $L^{\infty}(\mu)$ is contained in every $L^{q}(\mu)$, for $q \in[1,+\infty)$. However, these inclusions are not valid for a general measure $\mu$.

A function $f$ belonging to $L^{1}(\mu)$ is called integrable, while it is called square integrable if it belongs to $L^{2}(\mu)$. In general, we say that $f$ is $q$-integrable if it belongs to $L^{q}(\mu), q \in[1,+\infty)$. Let $\left(f_{n}\right)_{n \geq 1}$ be a sequence of measurable functions on the measure space $(\Omega, \mathscr{F}, \mu)$. We say that $\left(f_{n}\right)_{n \geq 1}$ converges ( $\mu$-a.e.) pointwise to the measurable function $f$ if

$$
\lim _{n \rightarrow+\infty}\left|f_{n}(x)-f(x)\right|=0
$$


for ( $\mu$-almost all) $x \in \Omega$. We write $f_{n} \longrightarrow f$ pointwise to mean that the sequence $\left(f_{n}\right)_{n \geq 1}$ converges pointwise to $f$. If the sequence $\left(f_{n}\right)_{n \geq 1}$ is monotonically increasing (resp., decreasing), i.e., $f_{n} \leq f_{n+1}$ (resp., $f_{n} \geq f_{n+1}$ ), we write $f_{n} \uparrow f$ (resp., $f_{n} \downarrow f$ ) to mean that it converges pointwise to $f$. If $\left(f_{n}\right)_{n \geq 1} \subseteq L^{q}(\mu)$ and $f \in L^{q}(\mu)$, we say that $\left(f_{n}\right)_{n \geq 1}$ converges to $f$ in $L^{q}(\mu)$ if

$$
\lim _{n \rightarrow+\infty}\left\|f_{n}-f\right\|_{q}=0 .
$$

It is important to establish under which conditions a sequence $\left(f_{n}\right)_{n \geq 1} \subseteq L^{q}(\mu)$ converging a.e. to a measurable function $f$ converges in fact to $f$ in $L^{q}(\mu)$. Now we state two classical theorems which answer this question: The theorem of Lebesgue on dominated convergence and the theorem of B. Levi on monotone convergence. We refer to Bauer (2001) II£.11 and II£.15. The following is the theorem of Lebesgue on dominated convergence.

1.1.3 Theorem. We fix $q \in[1,+\infty)$ and consider a sequence $\left(f_{n}\right)_{n \in \mathbb{N}} \subseteq L^{q}(\mu)$ such that $f_{n} \longrightarrow f \mu$-a.e. pointwise as $n \rightarrow+\infty$. If there exists a function $g \geq 0$ in $L^{q}(\mu)$ such that $\left|f_{n}\right| \leq g$, for every $n \in \mathbb{N}$, then $f \in L^{q}(\mu)$ and the convergence takes place also in $L^{q}(\mu)$.

Now we state the theorem of B. Levi on monotone convergence.

1.1.4 Theorem. Let $\left(f_{n}\right)_{n \in \mathbb{N}}$ be a monotone sequence of nonnegative functions such that $f_{n} \uparrow f$ pointwise as $n \rightarrow+\infty$. Then $f$ is measurable and $\mu\left(f_{n}\right) \uparrow \mu(f)$ as $n \rightarrow+\infty$.

\subsubsection{Monotone Class Theorems}

Monotone class theorems are of different kinds and they are present in the literature in several formulations. We consider only one formulation for sets and one for functions. We refer to Sharpe (1988) and He, Wang \& Yan (1992). We start with a monotone class theorem for systems of sets in the same form as He, Wang \& Yan (1992), Theorem 1.2. We say that a class $\mathscr{K}$ of subsets of $\Omega$ is a monotone class if for every monotone sequence $\left(A_{n}\right)_{n \in \mathbb{N}} \subseteq \mathscr{K}$ such that $A_{n} \uparrow A$ or $A_{n} \downarrow A$ as $n \rightarrow+\infty$, $A \in \mathscr{K}$.

1.1.5 Theorem (Monotone class theorem for sets). Let $\mathscr{F}$ be an algebra and $\mathscr{K}$ a monotone class of sets of $\Omega$ such that $\mathscr{F} \subseteq \mathscr{K}$. Then $\sigma(\mathscr{F}) \subseteq \mathscr{K}$.

For the formulation of the monotone class theorem for classes of functions we refer to Sharpe (1988), Appendix A0. Let $(\Omega, \mathscr{F})$ be a measurable space. We denote by $\mathbb{B}:=\mathbb{B}(\Omega, \mathbb{R})$ the set of bounded measurable functions on $(\Omega, \mathscr{F})$ into $(\mathbb{R}, \mathscr{B}(\mathbb{R}))$. If $\mathscr{K}$ is a linear subspace of $\mathbb{B}$ we say that it is a monotone vector space if $1=1_{\Omega} \in \mathscr{K}$ and if it is monotonically closed, that is, if $\left(f_{n}\right)_{n \in \mathbb{N}} \subseteq \mathscr{K}$ is such that $0 \leq f_{n} \leq f_{n+1}$, for all $n \in \mathbb{N}$ and $f \in \mathbb{B}$ is such that $f=\lim _{n \rightarrow+\infty} f_{n}$, then $f \in \mathscr{K}$. We observe that the limit $f$ belongs to $\mathbb{B}$ if and only if $\left(f_{n}\right)_{n \in \mathbb{N}}$ is uniformly bounded. A set $\mathscr{C} \subseteq \mathbb{B}$ is called a multiplicative class if it is closed with respect to the multiplication of two elements, meaning that if $h$ and $g$ belong to $\mathscr{C}$, then also their product $h g$ does.

1.1.6 Theorem (Monotone class theorem for functions). Let $\mathscr{K}$ be a monotone vector space and $\mathscr{C}$ a multiplicative class such that $\mathscr{C} \subseteq \mathscr{K}$ and $\sigma(\mathscr{C})=\mathscr{F}$. Then $\mathscr{K}=\mathbb{B}$. 


\subsubsection{Total Systems}

Let $(\Omega, \mathscr{F})$ be a measurable space and $\mu$ a measure on it. Given a system $\mathscr{T}$ of numerical functions on $(\Omega, \mathscr{F})$, we denote by $\operatorname{Span}(\mathscr{T})$ its linear hull. Now we fix $q$ in $[1,+\infty)$. If $\mathscr{T} \subseteq L^{q}(\mu)$ we denote by $\overline{\mathscr{T}}^{\left(L^{q}(\mu),\|\cdot\|_{q}\right)}$ the closure of $\mathscr{T}$ in $\left(L^{q}(\mu),\|\cdot\|_{q}\right)$. A system $\mathscr{T} \subseteq L^{q}(\mu)$ of functions is called total in $\left(L^{q}(\mu),\|\cdot\|_{q}\right)$ if its linear hull is dense, that is, $\overline{\operatorname{Span}(\mathscr{T})}\left(L^{q}(\mu),\|\cdot\|_{q}\right)=L^{q}(\mu)$. If $\mu$ is a finite measure, then the inclusions $L^{q}(\mu) \subseteq L^{p}(\mu), q \geq p$, hold and $L^{q}(\mu)$ is a total system in $\left(L^{p},\|\cdot\|_{p}\right)$. In particular, $L^{\infty}(\mu)$ is an example of total system in $L^{q}(\mu)$, for every $q \in[1,+\infty)$. The space $L^{q}(\mu)$ is called separable if there exists a countable system $\mathscr{T} \subseteq L^{q}(\mu)$ which is dense. If the $\sigma$-algebra $\mathscr{F}$ is separable, then the space $L^{q}(\Omega, \mathscr{F}, \mu)$ is also separable (cf., e.g., Brezis (1983)). It will be useful to determine when a system $\mathscr{T} \subseteq L^{q}(\mu)$ is total in $L^{q}(\mu)$. The following lemma is mathematically simple but it is of interest because it allows to reduce the problem of finding a total system in $L^{q}(\mu)$ to the one of finding a total system in $L^{q}\left(\mu^{g}\right)$, where $\mu^{g}$ is a certain finite measure.

1.1.7 Lemma. Let $g$ be such that $g(x) \neq 0$ for every $x \in \Omega$ and define the measure $\mu^{g}$ by

$$
\mathrm{d} \mu^{g}:=|g|^{q} \mathrm{~d} \mu .
$$

Then $\mu^{g}$ is a measure on $(\Omega, \mathscr{F})$. Furthermore:

(i) $\mu^{g}$ is a finite measure if and only if $g \in L^{q}(\mu), q \in[1,+\infty)$.

(ii) $f \in L^{q}\left(\mu^{g}\right)$ if and only if $f g \in L^{q}(\mu)$. Moreover, $\|f\|_{L^{q}\left(\mu^{g}\right)}=\|f g\|_{L^{q}(\mu)}$.

(iii) $\mathscr{T} \subseteq L^{q}\left(\mu^{g}\right)$ is total if and only if $g \mathscr{T}:=\{g f, f \in \mathscr{T}\}$ is total in $L^{q}(\mu)$.

(iv) Let $q=2$. Then $f, h \in L^{2}\left(\mu^{g}\right)$ are orthogonal if and only if $f g$ and $h g$ are orthogonal in $L^{2}(\mu)$.

Proof. Because of Radon-Nikodyn's Theorem, the measure $\mu^{g}$ is equivalent to $\mu$. We have $\mu^{g}(\Omega)=\mu\left(|g|^{q}\right)$ so $\mu^{g}$ is finite if and only if $g \in L^{q}(\mu)$. Moreover, $f \in L^{q}\left(\mu^{g}\right)$ if and only if $\mu^{g}\left(|f|^{q}\right)<+\infty$, that is if and only if $g f \in L^{q}(\mu)$ and (i) and (ii) are proven. To verify (iii) we first assume that $\mathscr{T}$ is total in $L^{q}\left(\mu^{g}\right)$ and observe that because of (ii) $f^{\prime}:=\frac{f}{g} \in L^{q}\left(\mu^{g}\right)$ if and only if $f \in L^{q}(\mu)$. Hence, if $f \in L^{q}(\mu)$ there exists a sequence $\left(f_{n}\right)_{n \in \mathbb{N}} \subseteq \operatorname{Span}(\mathscr{T})$ such that $f_{n} \longrightarrow f^{\prime}$ in $L^{q}\left(\mu^{g}\right)$ as $n \rightarrow+\infty$. From (ii) we get $g f_{n} \in L^{q}(\mu)$. So

$$
\int_{\Omega}\left|f-g f_{n}\right|^{q} \mathrm{~d} \mu=\int_{\Omega}\left|g\left(f^{\prime}-f_{n}\right)\right|^{q} \mathrm{~d} \mu=\int_{\Omega}\left|f^{\prime}-f_{n}\right|^{q} \mathrm{~d} \mu^{g} \longrightarrow 0, \quad n \rightarrow+\infty,
$$

and hence $g \mathscr{T} \subseteq L^{q}(\mu)$ is a total system. The converse implication can be shown in a similar way. To see (iv) it is enough to observe that for $f, h \in L^{2}\left(\mu^{g}\right)$ we have

$$
\mu^{g}(f h)=\mu\left((f h) g^{2}\right)=\mu((f g)(h g))
$$

and to use (ii) once again.

As an application of Theorem 1.1.6, we want to establish a general lemma stating sufficient conditions for a system $\mathscr{T} \subseteq L^{q}(\mu)$ of bounded functions to be total in $L^{q}(\mu)$, for $q \in[1,+\infty)$. We recall that we use the notation $\mathbb{B}:=\mathbb{B}(\Omega, \mathbb{R})$ to denote the space of bounded measurable functions on $(\Omega, \mathscr{F})$ into $(\mathbb{R}, \mathscr{B}(\mathbb{R}))$. 
1.1.8 Lemma. Let $\mathscr{T} \subseteq L^{q}(\mu)$ be a subset of $\mathbb{B}$. Then $\mathscr{T}$ is total in $L^{q}(\mu)$ if the following conditions are satisfied:

(i) $\mathscr{T}$ is stable under multiplication;

(ii) $\sigma(\mathscr{T})=\mathscr{F}$;

(iii) There exists a sequence $\left(h_{n}\right)_{n \in \mathbb{N}} \subseteq \operatorname{Span}(\mathscr{T})$ such that $h_{n} \geq 0$ and $h_{n} \uparrow 1$ pointwise as $n \rightarrow+\infty$.

Proof. First, we consider the case in which $\mu$ is a finite measure. In this case $\mathbb{B} \subseteq L^{q}(\mu)$ and it is dense in $\left(L^{q}(\mu),\|\cdot\|_{q}\right)$. We define $\mathscr{H}:=\overline{\operatorname{Span}(\mathscr{T})}\left(L^{q}(\mu),\|\cdot\|_{q}\right)$ and then $\mathscr{K}:=\mathscr{H} \cap \mathbb{B}$. Clearly, $\mathscr{K}$ is a closed linear space and $\mathscr{T} \subseteq \mathscr{K}$. By assumption, $h_{n} \uparrow 1$ pointwise as $n \rightarrow+\infty$ and by the finiteness of $\mu, 1 \in L^{q}(\mu)$. From the theorem of Lebesgue on dominated convergence (cf. Theorem 1.1.3) $h_{n}$ converges to 1 in $L^{q}(\mu)$ and so $1 \in \mathscr{K}$. Moreover, $\mathscr{K}$ is closed under monotone convergence of uniformly bounded nonnegative functions, as a consequence of the finiteness of $\mu$ and of Theorem 1.1.3. Consequently, $\mathscr{K}$ is a monotone class and by Theorem 1.1.6, we get $\mathscr{K}=\mathbb{B}$. Hence $\mathbb{B} \subseteq \mathscr{H}$ and since $\mathbb{B}$ is dense and $\mathscr{H}$ is closed, this yields $L^{q}(\mu)=\mathscr{H}$. Now we consider the case of a general measure $\mu$. For $f \in L^{q}(\mu)$ we put $\mathrm{d} \mu_{n}:=\left|h_{n}\right|^{q} \mathrm{~d} \mu, n \geq 1$, where $\left(h_{n}\right)_{n \in \mathbb{N}}$ is as in the assumptions of the lemma. Obviously, $\mu_{n}$ is a finite measure for every $n$. Moreover, $\mu_{n}\left(|f|^{q}\right)=\mu\left(\left|f h_{n}\right|^{q}\right) \leq \mu\left(|f|^{q}\right)<+\infty$ and hence $f \in L^{q}\left(\mu_{n}\right)$. We choose a sequence $\left(\varepsilon_{n}\right)_{n \in \mathbb{N}}$ such that $\varepsilon_{n}>0$ and $\varepsilon_{n} \downarrow 0$ as $n \rightarrow+\infty$. By the previous step, there exists a sequence $\left(g_{n}\right)_{n \in \mathbb{N}} \subseteq \operatorname{Span}(\mathscr{T})$ such that

$$
\int_{\Omega}\left|f h_{n}-g_{n} h_{n}\right|^{q} \mathrm{~d} \mu=\int_{\Omega}\left|f-g_{n}\right|^{q}\left|h_{n}\right|^{q} \mathrm{~d} \mu<\varepsilon_{n}, \quad n \geq 1 .
$$

The system $\mathscr{T}$ is stable under multiplication so $g_{n} h_{n} \in \operatorname{Span}(\mathscr{T})$. On the other side, $\left\|f-f h_{n}\right\|_{L^{q}(\mu)}=\left\||f|^{q}\left|1-h_{n}\right|^{q}\right\|_{L^{1}(\mu)} \longrightarrow 0$, as $n \rightarrow+\infty$. Indeed, $\left|1-h_{n}\right|^{q} \downarrow 0$ as $n \rightarrow+\infty$ and $|f|^{q}\left|1-h_{n}\right|^{q} \leq|f|^{q} \in L^{1}(\mu)$. Theorem 1.1.3 yields the result. Hence $g_{n} h_{n}$ converges to $f$ in $L^{q}(\mu)$ as $n \rightarrow+\infty$.

We remark that under the assumptions of Lemma 1.1.8, the measure $\mu$ is also $\sigma$-finite.

\subsection{Basics of Stochastic Processes}

Let $(\Omega, \tilde{\mathscr{F}})$ be a measurable space and let $\mathbb{P}$ be a probability measure on it. We call the measure space $(\Omega, \tilde{\mathscr{F}}, \mathbb{P})$ a probability space. By $\mathscr{N}(\mathbb{P})$ we denote the null sets of $\mathbb{P}$, i.e., $\mathscr{N}(\mathbb{P}):=\{A \subseteq \Omega: \exists B \in \tilde{\mathscr{F}}, A \subseteq B, \mathbb{P}(B)=0\}$. If $\mathscr{N}(\mathbb{P})$ is not contained in $\tilde{\mathscr{F}}$ we enlarge the $\sigma$-algebra by setting $\mathscr{F}:=\tilde{\mathscr{F}} \vee \mathscr{N}(\mathbb{P})$. We call $\mathscr{F}$ the completion of $\tilde{\mathscr{F}}$ (in itself) with respect to $\mathbb{P}$ or simply $\mathbb{P}$-completion of $\tilde{\mathscr{F}}$ and we say that $(\Omega, \mathscr{F}, \mathbb{P})$ is a complete probability space. If not otherwise specified, we assume a probability space to be complete. In the remaining of this chapter we assume that a complete probability space $(\Omega, \mathscr{F}, \mathbb{P})$ is fixed. A measurable mapping $X$ on $(\Omega, \mathscr{F})$ into $(\mathbb{R}, \mathscr{B}(\mathbb{R}))$ is called a random variable. We denote by $\mathbb{E}$ the expectation with respect to $\mathbb{P}$. If $\mathscr{G}$ is a sub- $\sigma$-algebra of $\mathscr{F}$, we denote by $\mathbb{E}[\cdot \mid \mathscr{G}]$ the conditional expectation with respect to $\mathscr{G}$. Sometimes we write $\mathbb{E}_{\mathbb{P}}$ or $\mathbb{E}_{\mathbb{P}}[\cdot \mid \mathscr{G}]$ to emphasize the dependence on the probability measure $\mathbb{P}$. 
Now we recall the notion of the uniform integrability. We say that a family $\mathscr{K} \subseteq L^{1}(\Omega, \mathscr{F}, \mathbb{P})$ is uniformly integrable if

$$
\sup _{X \in \mathscr{K}} \mathbb{E}\left[|X| 1_{\{|X| \geq N\}}\right] \longrightarrow 0 \quad \text { as } N \rightarrow+\infty .
$$

If $\mathscr{K}$ is dominated in $L^{1}(\mathbb{P})$, i.e., there exists $Y \in L^{1}(\mathbb{P})$ such that $|X| \leq Y, X \in \mathscr{K}$, then $\mathscr{K}$ is uniformly integrable. Clearly, any finite family of integrable random variables is uniformly integrable. La Vallée-Poussin's Theorem (cf. Meyer (1966), Theorem II.22) gives a characterization of the uniform integrability. It states that a family $\mathscr{K}$ of integrable random variables is uniformly integrable if and only if there exists a function $G$ on $[0,+\infty]$ into $[0,+\infty)$ such that: (i) $\frac{G(x)}{x}$ converges to $+\infty$ as $x \rightarrow+\infty$ and (ii) $\sup _{X \in \mathscr{K}} \mathbb{E}[G(|X|)]<+\infty$. Because of La Vallée-Poussin's Theorem, we can conclude that a family of random variables is uniformly integrable if it is $q$-integrable and bounded in $L^{q}(\mathbb{P}), q>1$.

A family $X=\left(X_{t}\right)_{t \geq 0}$ of $((\mathbb{R}, \mathscr{B}(\mathbb{R}))$-valued $)$ random variables is called a stochastic process or simply a process. The space $(\mathbb{R}, \mathscr{B}(\mathbb{R}))$ is the space of states of the process $X$ and the random variable $X_{t}$ is the state of the process $X$ at time $t \geq 0$. As observed in Dellacherie (1972), Chapter III a stochastic process $X$ can be interpreted as a mapping $X:(t, \omega) \longmapsto X(t, \omega)$ of $\mathbb{R}_{+} \times \Omega$ into $\mathbb{R}$. We use the notation $X_{t}(\omega):=X(t, \omega)$ and in most cases we omit $\omega$. If we endow the space $\mathbb{R}_{+} \times \Omega$ with the $\sigma$-algebra $\mathscr{B}\left(\mathbb{R}_{+}\right) \otimes \mathscr{F}$, we say that the process $X=\left(X_{t}\right)_{t \geq 0}$ is measurable if $X$ is a $\mathscr{B}\left(\mathbb{R}_{+}\right) \otimes \mathscr{F}$-measurable mapping of $\mathbb{R}_{+} \times \Omega$ into $\mathbb{R}$.

Let $X$ be a stochastic process. We call the mapping $t \mapsto X_{t}(\omega)$ path or trajectory of the process $X$. We say that the stochastic process $X$ is right-continuous with lefthand limit, and we use the abbreviation of French "continu à droite, limité à gauche" càdlàg, if every trajectory is càdlàg. Analogously, we say that $X$ is continuous or left-continuous if all its trajectories have this property. For a càdlàg process $X$, we define the random variable $X_{t-}$ for every $t>0$ as $X_{t-}:=\lim _{s \uparrow t} X_{s}$ which is finite. Adopting the convention $X_{0-}:=X_{0}$, we can introduce the process $X_{-}=\left(X_{t-}\right)_{t \geq 0}$ on the whole positive real line. With a càdlàg process $X$ we associate the process $\Delta X:=\left(\Delta X_{t}\right)_{t \geq 0}$ of jumps of $X$ by

$$
\Delta X_{t}:=X_{t}-X_{t-}, \quad t \geq 0 .
$$

A consequence of the convention $X_{0-}=X_{0}$ is that $\Delta X_{0}=0$. If $X$ is continuous stochastic process, then we have $X_{-}=X$ and $\Delta X=0$.

For two stochastic processes $X$ and $Y$, there exist different concepts of equality:

(i) $X$ is equal to $Y$ if $X_{t}(\omega)=Y_{t}(\omega)$ for every $t \geq 0$ and for every $\omega \in \Omega$.

(ii) $X$ and $Y$ are modifications if $\mathbb{P}\left(X_{t}=Y_{t}\right)=1$, for every $t \geq 0$.

(iii) $X$ and $Y$ are indistinguishable if $\mathbb{P}\left(X_{t}=Y_{t}\right.$, for every $\left.t \geq 0\right)=1$.

Two processes which are indistinguishable are also modifications. The converse is, in general, not true. However, if $X$ and $Y$ are a.s. right-continuous (resp., leftcontinuous), then they are indistinguishable if and only if they are modifications (cf. Dellacherie (1972), Theorem III.6).

Let $X$ be a càdlàg process. If, for a fixed $t>0, \Delta X_{t} \neq 0$ we say that the process $X$ has a fixed discontinuity at fixed time $t>0$. The convention $X_{0-}:=X_{0}$ ensures that a càdlàg process $X$ has no fixed discontinuity at time $t=0$. It is well 
known (cf., e.g., Appelbaum (2009), §2.9 p. 139]) that a càdlàg process has at most countably many fixed discontinuities, which means that the set $\left\{t \in \mathbb{R}_{+}: \Delta X_{t} \neq 0\right\}$ is at most countable. Moreover, for every fixed $\varepsilon>0$ and $T>0$ the set $\{t \in[0, T]$ : $\left.\left|\Delta X_{t}\right|>\varepsilon\right\}$ is finite. We say that a stochastic process $X$ is stochastically continuous (or continuous in probability) if

$$
\lim _{s \rightarrow t} \mathbb{P}\left(\left|X_{t}-X_{s}\right|>\varepsilon\right)=0, \quad t \geq 0, \quad \varepsilon>0 .
$$

For a càdlàg process which is stochastically continuous we have $\Delta X_{t}=0$ a.s. for every fixed $t \geq 0$. In other words, if a càdlàg process $X$ is stochastically continuous, then a.s. fixed discontinuities are excluded. This is a delicate point: The stochastic continuity of a càdlàg process does not mean that $\Delta X$ is indistinguishable from the null process but only that it is a modification of it.

\subsubsection{Filtrations and Stopping Times}

Let $(\Omega, \mathscr{F}, \mathbb{P})$ be a complete probability space and $\mathscr{N}(\mathbb{P})$ the class of the null sets of $\mathbb{P}$. A family $\tilde{\mathbb{F}}=\left(\tilde{\mathscr{F}}_{t}\right)_{t>0}$ of sub- $\sigma$-algebras of $\mathscr{F}$ which is increasing is called a filtration, that is, $\tilde{\mathscr{F}}_{t} \subseteq \mathscr{F}$ is a $\sigma$-algebra, $t \geq 0$, and $\tilde{\mathscr{F}}_{s} \subseteq \tilde{\mathscr{F}}_{t}, 0 \leq s \leq t$. A filtration $\mathbb{F}$ is called complete if $\mathscr{N}(\mathbb{P}) \subseteq \mathscr{F}_{t}$ for every $t \geq 0$. If a filtration $\tilde{\mathbb{F}}$ is not complete we can introduce the complete filtration $\mathbb{F}=\left(\mathscr{F}_{t}\right)_{t \geq 0}$ by setting $\mathscr{F}_{t}:=\tilde{\mathscr{F}}_{t} \vee \mathscr{N}(\mathbb{P})$, $t \geq 0$, and we call the filtration $\mathbb{F}$ the $\mathbb{P}$-completion of $\tilde{\mathbb{F}}$ (in $\mathscr{F}$ ). With a filtration $\tilde{\mathbb{F}}$ we associate the filtration $\tilde{\mathbb{F}}_{+}=\left(\tilde{\mathscr{F}}_{t+}\right)_{t \geq 0}$ by $\tilde{\mathscr{F}}_{t+}:=\cap_{\varepsilon>0} \tilde{\mathscr{F}}_{t+\varepsilon}$. The filtration $\tilde{\mathbb{F}}$ is called right-continuous if $\tilde{\mathbb{F}}=\tilde{\mathbb{F}}_{+}$, that is, if $\tilde{\mathscr{F}}_{t+}=\tilde{\mathscr{F}}_{t}, t \geq 0$. Note that $\mathscr{F}_{0+}=\mathscr{F}_{0}$. A filtration $\mathbb{F}$ which is complete and right-continuous is said to satisfy the usual conditions. Given a filtration $\tilde{\mathbb{F}}$ we can always associate to it a filtration $\mathbb{F}$ satisfying the usual conditions by setting $\mathscr{F}_{t}:=\tilde{\mathscr{F}}_{t+} \vee \mathscr{N}(\mathbb{P}), t \geq 0$. In the following of this work, if not otherwise specified, we shall always consider filtrations satisfying the usual conditions. By convention we set

$$
\mathscr{F}_{\infty}:=\mathscr{F} .
$$

1.2.1 Example. With a stochastic process $X$, we associate the $\sigma$-algebra $\tilde{\mathscr{F}}_{t}^{X}:=$ $\sigma\left(X_{s}, \quad 0 \leq s \leq t\right)$. We call the filtration $\tilde{\mathbb{F}}^{X}=\left(\tilde{\mathscr{F}}_{t}^{X}\right)_{t \geq 0}$ the filtration generated by $X$. Let $\mathscr{F}_{t}^{X}:=\tilde{\mathscr{F}}_{t}^{X} \vee \mathscr{N}(\mathbb{P})$ denote the $\mathbb{P}$-completion of $\tilde{\mathscr{F}}_{t}^{X}$. The filtration $\mathbb{F}^{X}:=\left(\mathscr{F}_{t}^{X}\right)_{t \geq 0}$ is the $\mathbb{P}$-completion of $\tilde{\mathbb{F}}^{X}$ (in $\mathscr{F}$ ). By $\tilde{\mathbb{F}}_{+}^{X}=\left(\tilde{\mathscr{F}}_{t+}^{X}\right)_{t \geq 0}$ we denote the smallest right-continuous filtration containing the filtration generated by $X$, i.e., $\tilde{\mathscr{F}}_{t+}^{X} \supseteq \tilde{\mathscr{F}}_{t}^{X}, t \geq 0$. In the sequel, the most relevant filtration associated with the stochastic process $X$ will be $\mathbb{F}_{+}^{X}$, i.e., the $\mathbb{P}$-completion in $\mathscr{F}$ of $\tilde{\mathbb{F}}_{+}^{X}$. The filtration $\mathbb{F}_{+}^{X}$ satisfies the usual conditions and we call it the natural filtration of $X$. With a stochastic process $X$ we associate the two $\sigma$-algebras $\tilde{\mathscr{F}}_{\infty}^{X}:=\sigma\left(X_{t}, \quad t \geq 0\right)$ and $\mathscr{F}_{\infty}^{X}:=\tilde{\mathscr{F}}_{\infty}^{X} \vee \mathscr{N}(\mathbb{P})$. This notation could cause ambiguities with the one introduced in (1.2). However, every time that we consider one of the filtrations associated with the process $X$, we also work on the probability space $\left(\Omega, \mathscr{F}_{\infty}^{X}, \mathbb{P}\right)$.

A process $X$ is adapted to a filtration $\mathbb{F}$ if $X_{t}$ is $\mathscr{F}_{t}$-measurable, for every $t \geq 0$. An adapted stochastic process $X$ which is càdlàg is $\mathscr{B}(\mathbb{R}) \otimes \mathscr{F}$-measurable. A stochastic process $X$ is always adapted to its generated filtration $\tilde{\mathbb{F}}^{X}$. Moreover, it is adapted 
to a filtration $\mathbb{F}$ if and only if $\tilde{\mathbb{F}}^{X} \subseteq \mathbb{F}$. If $X$ is adapted and càdlàg, then it is $\mathscr{B}(\mathbb{R}) \otimes \tilde{\mathscr{F}}_{\infty}^{X}$-measurable.

A mapping $T$ on $\Omega$ into $[0,+\infty]$ is called a stopping time if the set $\{T \leq t\}:=$ $\{\omega \in \Omega: T(\omega) \leq t\}$ is $\mathscr{F}_{t}$-measurable, for every $t \geq 0$. With a stopping time $T$ we associate the two $\sigma$-fields $\mathscr{F}_{T}:=\left\{A \in \mathscr{F}: A \cap\{T \leq t\} \in \mathscr{F}_{t}\right\}$ and $\mathscr{F}_{T-}:=$ $\mathscr{F}_{0} \vee\left\{A \cap\{t<T\}, A \in \mathscr{F}_{t}, t \geq 0\right\}$.

Let $S, T$ be two stopping times. We define the stochastic interval $[S, T)$ by

$$
[S, T):=\left\{(t, \omega) \in \mathbb{R}_{+} \times \Omega: S(\omega) \leq t<T(\omega)\right\} .
$$

The definitions of $(S, T],(S, T)$ and $[S, T]$ are analogous.

A stopping time $T$ is called predictable if there exists an increasing sequence $\left(T_{n}\right)_{n \in \mathbb{N}}$ of stopping times such that $T_{n} \uparrow T \omega$-wise as $n \rightarrow+\infty$ and $T_{n}<T$ on $\{T>0\}$. We now introduce two important $\sigma$-algebras on $\mathbb{R}_{+} \times \Omega$ : The optional $\sigma$-algebra and the predictable $\sigma$-algebra. We stress that these $\sigma$-algebras, as well as the notion of a stopping time, are always related with a filtration. We introduce the following $\sigma$-algebras of subsets of $\mathbb{R}_{+} \times \Omega$ :

$$
\mathscr{O}:=\sigma(\{[0, T): T \text { st. time }\}) \text { and } \mathscr{P}:=\sigma(\{[0, T): T \text { predictable st. time }\}),
$$

where the acronym st. stands for stopping. We call $\mathscr{O}=\mathscr{O}(\mathbb{F})$ the optional $\sigma$-algebra while $\mathscr{P}=\mathscr{P}(\mathbb{F})$ the predictable $\sigma$-algebra. We stress that both $\mathscr{O}$ and $\mathscr{P}$ are $\sigma$ algebras of the product space $\mathbb{R}_{+} \times \Omega$. A set $A \subseteq \mathbb{R}_{+} \times \Omega$ is said to be optional (resp., predictable) if it is $\mathscr{O}$-measurable (resp., $\mathscr{P}$-measurable). Analogously, a stochastic process $X$ is called optional (resp., predictable) if it is $\mathscr{O}$-measurable (resp., $\mathscr{P}$-measurable) as a mapping on $\mathbb{R}_{+} \times \Omega$. Obviously, the inclusions $\mathscr{P} \subseteq$ $\mathscr{O} \subseteq \mathscr{B}\left(\mathbb{R}_{+}\right) \otimes \mathscr{F}$ hold. Moreover, optional processes are also adapted (cf. Jacod \& Shiryaev (2000), Proposition I.1.21). The predictable $\sigma$-algebra plays a special role in the theory of stochastic integration. We recall that any left-continuous adapted process is predictable (cf. Dellacherie (1972) Theorem IV.22). It is important to note that for any stopping time $T$, the stochastic interval $[0, T]$ is a predictable set (cf. Dellacherie (1972), Theorem IV.3) and consequently the process $1_{[0, T]}$ is predictable. Moreover, if $X$ is a càdlàg process, the processes $\Delta X$ and $X_{-}$are optional and predictable, respectively. For the optionality of $\Delta X$ cf. Jacod \& Shiryaev (2000), Corollary I.1.25. The predictability of $X_{-}$is immediate because it is a left-continuous adapted process.

Let $X$ be a stochastic process and $T$ a stopping time with values in $[0,+\infty]$. On $\{T<+\infty\}$, we define the random variable $X_{T}$ by $X_{T}(\omega):=X_{T(\omega)}(\omega)=X(T(\omega), \omega)$ and, consequently, the stochastic process $X^{T}=\left(X_{t}^{T}\right)_{t \geq 0}$ by $X_{t}^{T}:=X_{t \wedge T}, t \geq 0$, where the symbol " $\wedge$ " denotes the minimum function. We say that $X^{T}$ is the stopped process at time $T$. If $X$ is an optional (resp., predictable) process, then the stopped process $X^{T}$ is optional (resp., predictable) (cf. Jacod \& Shiryaev (2000), Proposition I.1.21 and I.2.4). A class $\mathscr{C}$ of processes is called stable under stopping if for every $X \in \mathscr{C}$ the stopped process $X^{T}$ belongs again to $\mathscr{C}$, for every stopping time $T$. The procedure that we are going to describe will be used several times in this work and it is well-known as localization. If $\mathscr{C}$ is a class of processes, we denote by $\mathscr{C}_{\text {loc }}$ the localized class, defined as such: A process $X$ belongs to $\mathscr{C}_{\text {loc }}$ if and only 
if there exists an increasing sequence $\left(T_{n}\right)_{n \in \mathbb{N}}$ of stopping times (depending on $X$ ) such that $T_{n} \uparrow+\infty$ a.s. and each stopped process $X^{T_{n}}$ belongs to $\mathscr{C}$. The sequence $\left(T_{n}\right)_{n \in \mathbb{N}}$ is called a localizing sequence or a reducing sequence for $X$ (relative to $\mathscr{C}$ ). If for example $\mathscr{C}$ is the class of bounded processes, the class of locally bounded processes $\mathscr{C}_{\text {loc }}$ is the class of processes $X$ such that there exists a reducing sequence $\left(T_{n}\right)_{n \in \mathbb{N}}$ such that $X^{T_{n}}$ is bounded. Let $X$ be a càdlàg process. The process $\left(X_{t-}\right)_{t \geq 0}$ is locally bounded if and only if $X_{0-}:=X_{0}$ is a bounded random variable. If $X$ is a càdlàg process, we can write

$$
X=X_{-}+\Delta X \text {. }
$$

Therefore a càdlàg process $X$ with bounded jumps such that $X_{0}$ is bounded is locally bounded.

In the sequel of this chapter we fix a complete probability space $(\Omega, \mathscr{F}, \mathbb{P})$ and a filtration $\mathbb{F}$ satisfying the usual conditions.

\subsubsection{Martingales}

A martingale (resp., submartingale; resp., supermartingale) relative to the filtration $\mathbb{F}$ is an adapted process $X$, such that $X_{t} \in L^{1}(\mathbb{P})$ for every $t \geq 0$ and that for all $0 \leq s \leq t$, we have

$$
\left.\mathbb{E}\left[X_{t} \mid \mathscr{F}_{s}\right]=X_{s} \quad \text { (resp., } \mathbb{E}\left[X_{t} \mid \mathscr{F}_{s}\right] \geq X_{s} ; \text { resp., } \mathbb{E}\left[X_{t} \mid \mathscr{F}_{s}\right] \leq X_{s}\right) .
$$

Sometimes, for shortness, we simply say that a martingale (resp., a submartingale; resp., a supermartingale) relative to a filtration $\mathbb{F}$ is an $\mathbb{F}$-martingale (resp., $\mathbb{F}$-submartingale; resp., $\mathbb{F}$-supermartingale) to emphasize the filtration. Because the filtration $\mathbb{F}$ satisfies the usual conditions, every $\mathbb{F}$-martingale admits a càdlàg modification which is again an $\mathbb{F}$-martingale (cf., e.g., Meyer (1966), Chapter VI or He, Wang \& Yan (1992), Chapter II). If not otherwise specified, we always consider càdlàg martingales.

We say that a process $X$ has a terminal variable $X_{\infty}$ if $X_{t}$ converges a.s. to a limit $X_{\infty}$ as $t \uparrow+\infty$. If $T$ is a stopping time, the random variable $X_{T}:=X_{T(\omega)}(\omega)$ is, in general, only defined on $\{T<+\infty\}$. If the process $X$ admits the terminal variable $X_{\infty}$, the random variable $X_{T}$ is defined also on $\{T=+\infty\}$.

We denote by $\mathscr{M}$ the class of uniformly integrable martingales, i.e., the class of all martingales $M$ such that the family of random variables $\left(M_{t}\right)_{t \geq 0}$ is uniformly integrable. By $\mathscr{M}_{0}$, we denote the subset of uniformly integrable martingales starting at 0 . The terminal value $M_{\infty}$ of a uniformly integrable martingale exists and $M_{t}$ converges in $L^{1}(\mathbb{P})$ to $M_{\infty}$. Furthermore, for every stopping times $T$, $M_{T}=\mathbb{E}\left[M_{\infty} \mid \mathscr{F}_{T}\right]$. For a proof of these facts, cf. Jacod \& Shiryaev (2000), Theorem I.1.42. Doob's Stopping Theorem (cf. Jacod \& Shiryaev (2000), Theorem I.1.39) implies that for every stopping time $T$ the random variable $M_{T}, M \in \mathscr{M}$, is integrable and, if $S$ is also a stopping time, we have $\mathbb{E}\left[M_{T} \mid \mathscr{F}_{S}\right]=M_{S}$ on $\{S \leq T\}$. A first consequence of these facts is that $\mathscr{M}$ is stable under stopping. Indeed, if $M \in \mathscr{M}$ and $T$ is a stopping time, we have that $T \wedge t$ is a stopping time and that $M_{\infty}$ and $M_{T \wedge t}$ are integrable, $t \geq 0$. Moreover,

$$
\left|M_{t}^{T}\right|=\left|M_{T \wedge t}\right|=\left|\mathbb{E}\left[M_{\infty} \mid \mathscr{F}_{T \wedge t}\right]\right| \leq \mathbb{E}\left[\left|M_{\infty}\right| \mid \mathscr{F}_{T \wedge t}\right],
$$


where we used Jensen's inequality in the last passage. Because of He, Wang \& Yan (1992), Theorem 1.8, the right-hand side in the previous estimation is uniformly integrable and so $M^{T}=\left(M_{t}^{T}\right)_{t \geq 0}$ is uniformly integrable. By Doob's Stopping Theorem, we get $M_{t}=\mathbb{E}\left[M_{T} \mid \mathscr{F}_{t}\right]$ on $\{t \leq T\}$ and, by the properties of the conditional expectation, $\mathbb{E}\left[M_{T} \mid \mathscr{F}_{t}\right]=M_{T}$ on $\{t>T\}$. Hence $M_{t}^{T}=\mathbb{E}\left[M_{T} \mid \mathscr{F}_{t}\right], t \geq 0$, and so the process $M^{T}$ is an $\mathbb{F}$-martingale. Now we state a lemma which gives necessary and sufficient conditions for an adapted process $X$ to be a uniformly integrable martingale. For the proof cf. Jacod \& Shiryaev (2000), Lemma I.1.44.

1.2.2 Lemma. Let $X$ be an adapted process with a terminal random variable $X_{\infty}$. Then $X$ is a uniformly integrable martingale (non necessarily càdlàg) if and only if for every stopping time $T$ the random variable $X_{T}$ is integrable and $\mathbb{E}\left[X_{T}\right]=\mathbb{E}\left[X_{0}\right]$.

By localization we can introduce from $\mathscr{M}$ (resp., $\mathscr{M}_{0}$ ) the space $\mathscr{M}_{\text {loc }}$ (resp., $\mathscr{M}_{\text {loc, }, 0}$ ). A local martingale (resp., a local martingale starting at 0 ) will be an element of $\mathscr{M}_{\text {loc }}$ (resp., of $\mathscr{M}_{\mathrm{loc}, 0}$ ). We observe that the classes $\mathscr{M}_{\mathrm{loc}}$ and $\mathscr{M}_{\mathrm{loc}, 0}$ are stable under stopping. Sometimes, we shall use the notation $\mathscr{M}_{\text {loc }, 0}(\mathbb{P})$ (resp., $\mathscr{M}_{\text {loc }, 0}(\mathbb{F}) ;$ resp. $\mathscr{M}_{\text {loc }, 0}(\mathbb{P}, \mathbb{F})$ ) to stress the probability measure $\mathbb{P}$ (resp., the filtration $\mathbb{F}$; resp., the probability measure $\mathbb{P}$ and the filtration $\mathbb{F}$ ) with respect to which we consider the space $\mathscr{M}_{\text {loc }}$.

A sufficient condition to ensure that a local martingale $M$ is a true uniformly integrable martingale is that $M$ is bounded in $L^{1}(\mathbb{P})$. This is an easy consequence of the theorem of Lebesgue on monotone convergence. Indeed, if $\left(T_{n}\right)_{n \geq 1}$ is a sequence of stopping times localizing $M$ to $\mathscr{M}$ and we assume that there exists and integrable random variable $X \geq 0$ such that $\left|M_{t}^{T_{n}}\right| \leq X$, then we can apply the theorem of Lebesgue on dominated convergence to get, for every $0 \leq s \leq t$,

$$
M_{s}=\lim _{n \rightarrow+\infty} M_{s}^{T_{n}}=\lim _{n \rightarrow+\infty} \mathbb{E}\left[M_{t}^{T_{n}} \mid \mathscr{F}_{s}\right]=\mathbb{E}\left[\lim _{n \rightarrow+\infty} M_{t}^{T_{n}} \mid \mathscr{F}_{s}\right]=\mathbb{E}\left[M_{t} \mid \mathscr{F}_{s}\right],
$$

meaning that $M$ is a true martingale. The uniform integrability follows because $M_{t} \in L^{1}(\mathbb{P})$ converges to $M_{\infty}$ a.s. as $t \rightarrow+\infty$. A new application of the theorem of Lebesgue on dominated convergence implies that $M_{\infty}$ belongs to $L^{1}(\mathbb{P})$. Because $M$ is a martingale, $|M|$ is a submartingale and so we have $\left|M_{t}\right| \leq \mathbb{E}\left[\left|M_{\infty}\right| \mid \mathscr{F}_{t}\right], t \geq 0$, and by He, Wang \& Yan (1992), Theorem 1.8, we get that $M$ is uniformly integrable.

We conclude this part with a lemma showing the stability of the martingale property under $L^{1}(\mathbb{P})$-convergence.

1.2.3 Lemma. Let $\left(M^{n}\right)_{n \geq 1}$ be a sequence of $\mathbb{F}$-martingales and $M$ a stochastic process such that $M_{t}$ is integrable, $t \geq 0$. If $M_{t}^{n}$ converges in $L^{1}(\mathbb{P})$ to $M_{t}$ for every $t \geq 0$, then the process $M$ is an $\mathbb{F}$-martingale (non necessarily càdlàg).

Proof. Let $0 \leq s \leq t$ and $A \in \mathscr{F}_{s}$. Then the random variable $1_{A} M_{t}^{n}$ converges in $L^{1}(\mathbb{P})$ to $1_{A} M_{t}$ as $n \rightarrow+\infty$ and this implies that $\mathbb{E}\left[1_{A} M_{t}^{n}\right]$ converges to $\mathbb{E}\left[1_{A} M_{t}\right]$ as $n \rightarrow+\infty$, for every $t \geq 0$. Therefore, because of $\mathbb{E}\left[1_{A} M_{t}^{n}\right]=\mathbb{E}\left[1_{A} M_{s}^{n}\right]$, we get $\mathbb{E}\left[1_{A} M_{t}\right]=\mathbb{E}\left[1_{A} M_{s}\right]$ and hence $M$ is a martingale.

\subsubsection{Increasing Processes}

We denote by $\mathscr{V}^{+}$(resp., by $\mathscr{V}$ ) the set of all real-valued processes $A$ that are càdlàg, adapted, with $A_{0}=0$ and whose paths are non-decreasing (resp., have finite 
variation on each finite interval $[0, t])$. We say that a process in $\mathscr{V}^{+}($resp., in $\mathscr{V})$ is an increasing process (resp., a process of finite variation).

For a process $A \in \mathscr{V}$, by $\operatorname{Var}(A)=\left(\operatorname{Var}(A)_{t}\right)_{t \geq 0}$ we denote the associated variation process, that is, the process such that $\operatorname{Var}(A)_{t}(\omega)$ is the total variation of the function $s \mapsto A_{s}(\omega)$ on the interval $[0, t]$. Of course, $\operatorname{Var}(A)=A$ if $A \in \mathscr{V}^{+}$. In Jacod \& Shiryaev (2000), Proposition I.3.3 the following important relation between $\mathscr{V}$ and $\mathscr{V}^{+}$is established: If $A \in \mathscr{V}$, then there exists a unique pair of processes $B, C \in \mathscr{V}^{+}$ such that $A=B-C$ and $\operatorname{Var}(A)=B+C$ (hence, $\operatorname{Var}(A) \in \mathscr{V}^{+}$and $\mathscr{V}=\mathscr{V}^{+} \ominus \mathscr{V}^{+}$). Moreover, if $A$ is predictable, then $B, C$ and $\operatorname{Var}(A)$ are also predictable.

For a process $A \in \mathscr{V}^{+}$the function $t \mapsto A_{t}(\omega)$ is a measure-generating function, that is, it is the distribution function of a measure,say $\mu_{\omega}^{A}$, defined on $\mathbb{R}_{+}$by

$$
\mu_{\omega}^{A}([0, t]):=A_{t}(\omega), \quad t \in \mathbb{R}_{+}, \quad \omega \in \Omega,
$$

which is a locally finite measure.

The stochastic integral with respect to $A \in \mathscr{V}^{+}$of a measurable process $H$ is defined as a Stiltjes-Lebesgue integral, i.e., by fixing $\omega \in \Omega$ and defining the integral pathwise with respect to the trajectory $t \mapsto A_{t}(\omega)$. We say that a measurable process $H$ is integrable with respect to $A$ if $\int_{0}^{t}\left|H_{s}(\omega)\right| \mu_{\omega}^{A}(\mathrm{~d} s)<+\infty$ for every $t \geq 0$ and for every $\omega \in \Omega$. If $H$ is integrable with respect to $A$ by

$$
\int_{0}^{t} H_{s}(\omega) \mathrm{d} A_{s}(\omega):=\int_{0}^{t} H_{s}(\omega) \mu_{\omega}^{A}(\mathrm{~d} s), \quad t \geq 0,
$$

we denote the integral of $H$ with respect to $A$ up to time $t$. We introduce the integral process $H \cdot A=\left(H \cdot A_{t}\right)_{t \geq 0}$ by

$$
H \cdot A_{t}(\omega):= \begin{cases}\int_{0}^{t} H_{s}(\omega) \mathrm{d} A_{s}(\omega), & \text { if } \int_{0}^{t}\left|H_{s}(\omega)\right| \mathrm{d} A_{s}(\omega)<+\infty \\ +\infty, & \text { otherwise. }\end{cases}
$$

If $A \in \mathscr{V}$, we can introduce the integral for measurable processes in a similar way. Indeed, there exist two unique processes $B, C \in \mathscr{V}^{+}$such that $A=B-C$ and $\operatorname{Var}(A)=B+C$. In this case, we say that a measurable process $H$ is integrable with respect to $A$ if it is integrable with respect to $\operatorname{Var}(A)$ and we introduce the integral process $H \cdot A=\left(H \cdot A_{t}\right)_{t \geq 0}$ by

$$
H \cdot A_{t}(\omega):= \begin{cases}\int_{0}^{t} H_{s}(\omega) \mathrm{d} A_{s}(\omega), & \text { if } \int_{0}^{t}\left|H_{s}(\omega)\right| \mathrm{d} \operatorname{Var}(A)_{s}(\omega)<+\infty \\ +\infty, & \text { otherwise }\end{cases}
$$

If $H$ is a measurable process (resp., nonnegative) which is integrable with respect to $A \in \mathscr{V}$ (resp., $A \in \mathscr{V}^{+}$), then the process $H \cdot A$ belongs to $\mathscr{V}$ (resp., $\mathscr{V}^{+}$). If moreover $A$ and $H$ are predictable, then $H \cdot A$ is predictable (cf. Jacod \& Shiryaev (2000), Proposition I.3.5). We notice that locally bounded measurable processes are always integrable with respect to $A \in \mathscr{V}$. Indeed, if $H$ is a locally bounded measurable process and $\left(T_{n}\right)_{n \in \mathbb{N}}$ is a reducing sequence, say such that $\left|H^{T_{n}}\right| \leq c_{n}$, 
we have, for every $n \geq 1$ and every fixed $t \geq 0,\left|H^{T_{n}}\right| \cdot \operatorname{Var}(A)_{t} \leq c_{n} \operatorname{Var}(A)_{t}<+\infty$. On the other side, $T_{n} \uparrow+\infty$. Hence, for every fixed $t \geq 0$, there exists $n(t) \in \mathbb{N}$ such that $T_{n} \geq t$ for every $n \geq n(t)$ and so $|H| \cdot \operatorname{Var}(A)_{t} \leq c_{n} \operatorname{Var}(A)_{t}<+\infty, n \geq n(t)$.

Now we introduce two other classes of processes.

(i) $\mathscr{A}^{+}:=\left\{A \in \mathscr{V}^{+}: \mathbb{E}\left[A_{\infty}\right]<+\infty\right\}$ : set of integrable processes.

(ii) $\mathscr{A}:=\left\{A \in \mathscr{V}: \mathbb{E}\left[\operatorname{Var}(A)_{\infty}\right]<+\infty\right\}$ : set of processes integrable variation.

We note that from the classes $\mathscr{A}^{+}, \mathscr{A}, \mathscr{V}^{+}$and $\mathscr{V}$ the corresponding localized classes $\mathscr{A}_{\text {loc }}^{+}, \mathscr{A}_{\text {loc }}, \mathscr{V}_{\text {loc }}^{+}$and $\mathscr{V}_{\text {loc }}$ can be introduced. We have $\mathscr{V}_{\text {loc }}^{+}=\mathscr{V}^{+}$and $\mathscr{V}_{\text {loc }}=\mathscr{V}$. All the previous classes of processes are stable under stopping. Moreover, we have $\mathscr{A}=\mathscr{A}^{+} \ominus \mathscr{A}^{+}, \mathscr{A}_{\mathrm{loc}}=\mathscr{A}_{\mathrm{loc}}^{+} \ominus \mathscr{A}_{\mathrm{loc}}^{+}$and the following inclusions $\mathscr{A}^{+} \subseteq \mathscr{A}_{\mathrm{loc}}^{+} \subseteq \mathscr{V}^{+}$, $\mathscr{A} \subseteq \mathscr{A}_{\text {loc }} \subseteq \mathscr{V}$ hold. We observe that the set of local martingales of finite variation is contained in $\mathscr{A}_{\text {loc }}$, i.e., $\mathscr{A}_{\text {loc }} \supseteq \mathscr{M}_{\text {loc }} \cap \mathscr{V}$ (cf. Jacod \& Shiryaev (2000), Lemma I.3.11). Now we formulate a very important theorem which states the existence of the compensator for processes in $\mathscr{A}_{\mathrm{loc}}^{+}$. We refer to Jacod \& Shiryaev (2000), Theorem I.3.17.

1.2.4 Theorem. Let $A \in \mathscr{A}_{\mathrm{loc}}^{+}$. There exists a process, called the compensator of $A$ and denoted by $A^{p}$, which is unique up to an evanescent set and which is characterized by being a predictable process in $\mathscr{A}_{\mathrm{loc}}^{+}$meeting any one of the following three equivalent statements:

(i) $A-A^{p}$ is a local martingale;

(ii) $\mathbb{E}\left[A_{T}^{p}\right]=\mathbb{E}\left[A_{T}\right]$ for all stopping times $T$;

(iii) $\mathbb{E}\left[H \cdot A_{\infty}^{p}\right]=\mathbb{E}\left[H \cdot A_{\infty}\right]$ for all nonnegative predictable process $H$.

Theorem 1.2.4 can be extended to processes of locally integrable variation as in Jacod \& Shiryaev (2000), Theorem I.3.18. If $A \in \mathscr{A}_{\mathrm{loc}}$ the compensator of $A$ is denoted again by $A^{p}$ and it is the unique (up to an evanescent set) predictable process of $\mathscr{A}_{\text {loc }}$ such that $A-A^{p}$ is a local martingale. Moreover, for each predictable process $H$ such that $H \cdot A \in \mathscr{A}_{\text {loc }}$, it holds $H \cdot A^{p} \in \mathscr{A}_{\text {loc }}$ and $(H \cdot A)^{p}=H \cdot A^{p}$. In particular $H \cdot A-H \cdot A^{p}$ is a local martingale. We conclude this section with the following proposition.

1.2.5 Proposition. (i) $A \in \mathscr{A}_{\mathrm{loc}} \cap \mathscr{M}_{\mathrm{loc}}$ if and only if $A^{p}=0$.

(ii) $A \in \mathscr{M}_{\mathrm{loc}} \cap \mathscr{A}$ if and only if $A \in \mathscr{M} \cap \mathscr{A}$.

(iii) If $A \in \mathscr{M}_{\mathrm{loc}} \cap \mathscr{V}$ (hence $A \in \mathscr{M}_{\mathrm{loc}} \cap \mathscr{A}_{\mathrm{loc}}$ ) and $H$ is a predictable process such that $H \cdot A \in \mathscr{A}_{\mathrm{loc}}$, then $H \cdot A \in \mathscr{M}_{\mathrm{loc}}$.

Proof. (i) is a consequence of the uniqueness of the Doob-Meyer decomposition of submartingales (cf. Jacod \& Shiryaev (2000), Theorem I.3.15 and Corollary I.3.16). To see (ii) it is enough to observe that if $A \in \mathscr{M}_{\mathrm{loc}} \cap \mathscr{A}$, because of $\left|A_{t}\right| \leq \operatorname{Var}(A)_{\infty}$ and of $\operatorname{Var}(A)_{\infty} \in L^{1}(\mathbb{P})$, then $A$ is a local martingale bounded in $L^{1}(\mathbb{P})$, hence a uniformly integrable martingale. The converse implication is trivial. Now we show (iii). If $H$ is a predictable process which is integrable with respect to $A$ and such that $H \cdot A \in \mathscr{A}_{\mathrm{loc}}$, from the properties of the compensator for processes of locally integrable variation we get $(H \cdot A)^{p}=H \cdot A^{p}$. But $A^{p}=0$ because of (i). A new application of (i) yields $(H \cdot A) \in \mathscr{M}_{\text {loc }}$ and this concludes the proof. 


\subsubsection{The Poisson process}

In this part we want to discuss an important example of increasing process: The Poisson process. As a first step, we introduce simple point processes. Let $N \in \mathscr{V}^{+}$. We say that it is a simple point process relative to $\mathbb{F}$ if

(i) $N$ is $\mathbb{F}$-adapted;

(ii) $N$ takes values in $\mathbb{N}$ a.s.;

(iii) $\Delta N$ takes values in $\{0,1\}$ a.s.

We observe that a simple point process belongs to $\mathscr{A}_{\text {loc }}^{+}$and is even a locally bounded process (it has bounded jumps, starts at 0 and is càdlàg).

1.2.6 Definition. Let $N$ be a simple point process relative to $\mathbb{F}$. We say that it is a Poisson process relative to $\mathbb{F}$ if:

(i) $\mathbb{E}\left[N_{t}\right]<+\infty$, for every $t \in \mathbb{R}_{+}$;

(ii) The function $a(t):=\mathbb{E}\left[N_{t}\right]$, called intensity function of $N$, is continuous;

(iii) $N_{t}-N_{s}$ is independent of $\mathscr{F}_{s}$, for every $0 \leq s<t$.

If $N$ is a Poisson process relative to $\mathbb{F}$ with intensity function $a(\cdot)$ of the form $a(t)=\gamma t, t \geq 0, \gamma>0$, we say that $N$ is a homogeneous Poisson process (with parameter $\gamma$ ) relative to $\mathbb{F}$.

We notice that a Poisson process $N$ is stochastically continuous. Indeed, by definition it is a càdlàg process end hence it is right-continuous. To show that it is stochastically continuous it is enough to prove that it is stochastically continuous from the left. But this is an immediate consequence of Markov's inequality. For any $\varepsilon>0$ and $0 \leq s \leq t$ we have $N_{t} \geq N_{s}$ and

$$
\lim _{s \uparrow t} \mathbb{P}\left[\left(N_{t}-N_{s}\right) \geq \varepsilon\right] \leq \frac{1}{\varepsilon} \lim _{s \uparrow t} \mathbb{E}\left[N_{t}-N_{s}\right]=\frac{1}{\varepsilon} \lim _{s \uparrow t}(a(t)-a(s))=0,
$$

where in the first passage we applied Markov's inequality and in the last equality we used the continuity of the intensity function $a(\cdot)$.

A nice characterization for a simple point process relative to $\mathbb{F}$ to be a Poisson process relative to $\mathbb{F}$ in term of its compensator can be given. This result will play a key role in the further development. For this reason we formulate it here as a theorem but we refer to Jacod \& Shiryaev (2000), Proposition I.3.27 and Theorem II.4.5 for the proof.

1.2.7 Theorem. A simple point process $N$ relative to $\mathbb{F}$ is a Poisson process relative to $\mathbb{F}$ if and only if its compensator $N^{p}$ is a deterministic continuous increasing function, say $a(\cdot)$.

Theorem 1.2.7 can be reformulated saying that a simple point process is a Poisson process if and only if there exists a deterministic continuous increasing function $a(\cdot)$ such that $N-a(\cdot)$ is a local martingale. If $N$ is a Poisson process relative to $\mathbb{F}$ with intensity function $a(\cdot)$, then its characteristic function is given by

$$
\mathbb{E}\left[\exp \left(\left(i u\left(N_{t}-N_{s}\right)\right)\right]=\exp \left(\left(\mathrm{e}^{i u}-1\right)(a(t)-a(s))\right), \quad 0 \leq s \leq t, \quad u \in \mathbb{R} .\right.
$$

(cf. Jacod \& Shiryaev (2000), Theorem II.4.5). Note that from (1.3) one immediately deduces that if $N$ is a Poisson process relative to $\mathbb{F}$, then the random variable $N_{t}$ is Poisson distributed with parameter $a(t), t \geq 0$. 


\subsubsection{Locally Square Integrable Martingales}

We denote by $\mathscr{H}^{2}$ the class of square integrable martingales, that is, we put

$$
\mathscr{H}^{2}:=\left\{M \in \mathscr{M}_{\mathrm{loc}}: \mathbb{E}\left[\sup _{t \geq 0}\left(M_{t}\right)^{2}\right]<+\infty\right\} .
$$

The space $\mathscr{H}_{\text {loc }}^{2}$ of locally square integrable local martingales is introduced from $\mathscr{H}^{2}$ in the usual way. Clearly, any locally bounded local martingale belongs to $\mathscr{H}_{\text {loc }}^{2}$. In particular, any continuous local martingale is locally square integrable. The space of local martingales $\mathscr{H}^{2}$ is a subspace of $\mathscr{M}$. Indeed, if $M \in \mathscr{H}^{2}$, then $\left|M_{t}\right|$ is dominated in $L^{2}(\mathbb{P})$ by $\sup _{t \geq 0}\left|M_{t}\right|$. Therefore, if $M \in \mathscr{H}^{2}$, the terminal variable $M_{\infty}$ is defined and, because of $\mathbb{E}\left[M_{\infty}^{2}\right] \leq \mathbb{E}\left[\sup _{t>0}\left(M_{t}\right)^{2}\right]<+\infty$, it belongs to $L^{2}(\mathbb{P})$. For $M, N \in \mathscr{H}^{2}$ the following expressions

$$
(M, N)_{\mathscr{H}^{2}}:=\mathbb{E}\left[M_{\infty} N_{\infty}\right], \quad\|M\|_{2}^{*}:=\left\|M_{\infty}\right\|_{L^{2}(\mathbb{P})}
$$

define a scalar product and a norm, respectively. The space $\left(\mathscr{H}^{2},\|\cdot\|_{2}^{*}\right)$ can be identified with $\left(L^{2}(\mathbb{P}),\|\cdot\|_{L^{2}(\mathbb{P})}\right)$ as it follow: With every $M \in \mathscr{H}^{2}$ we associate the terminal variable $M_{\infty} \in L^{2}(\mathbb{P})$. This yields $\left(\mathscr{H}^{2},\|\cdot\|_{2}^{*}\right)$ is a Hilbert space.

The space $\mathscr{H}_{\text {loc }}^{2}$ is especially important because of the following well-known result (cf. Jacod \& Shiryaev (2000), Theorem I.4.2): For any $M$ and $N$ belonging to $\mathscr{H}_{\mathrm{loc}}^{2}$, there exists a predictable process $\langle M, N\rangle \in \mathscr{V}$, called point brackets (or predictable process of finite variation) associated to $M$ and $N$, which is unique up to an evanescent set, such that $M N-\langle M, N\rangle \in \mathscr{M}_{\text {loc }}$. The so-called polarization identity holds:

$$
\langle M, N\rangle=\frac{1}{4}(\langle M+N, M+N\rangle-\langle M-N, M-N\rangle) .
$$

If $M, N \in \mathscr{H}^{2}$, then $\langle M, N\rangle \in \mathscr{A}$ and $M N-\langle M, N\rangle \in \mathscr{M}$. Furthermore, the identity $\langle M, N\rangle=\left\langle M-M_{0}, N-N_{0}\right\rangle$ holds and $\langle M, M\rangle$ belongs to $\mathscr{V}^{+}$. The next relation explains the behavior of the point brackets with respect to the stopping procedure (cf. He, Wang \& Yan (1992), Theorem 6.31). For every stopping time $T$ it follows that

$$
\left\langle M, N^{T}\right\rangle=\left\langle M^{T}, N\right\rangle=\left\langle M^{T}, N^{T}\right\rangle=\langle M, N\rangle^{T} .
$$

Furthermore, because of the definition of the point brackets, we have

$$
(M, N)_{\mathscr{H}^{2}}:=\mathbb{E}\left[\langle M, N\rangle_{\infty}\right]+\mathbb{E}\left[M_{0} N_{0}\right], \quad M, N \in \mathscr{H}^{2} .
$$

\subsubsection{The Wiener process}

One of the most important locally square integrable martingales, but probably one of most important stochastic processes, is the Wiener process. In this short section we collect some properties of the Wiener process which will be useful in this work.

1.2.8 Definition. A continuous adapted process $W$ such that $W_{0}=0$ is called a Wiener process (or a Brownian motion) relative to $\mathbb{F}$ if:

(i) $\mathbb{E}\left[W_{t}^{2}\right]<+\infty$ and $\mathbb{E}\left[W_{t}\right]=0$, for every $t \geq 0$;

(ii) $W_{t}-W_{s}$ is independent of $\mathscr{F}_{s}$, for all $0 \leq s \leq t$.

The function $\sigma^{2}(t):=\mathbb{E}\left[W_{t}^{2}\right]$ is called the variance function of $W$. If $\sigma^{2}(t)=t$, we say that $W$ is a standard Wiener process. 
It is immediate to see that a Wiener process relative to $\mathbb{F}$ is an $\mathbb{F}$-martingale. The point brackets associated with $W$ are given by $\langle W, W\rangle_{t}=\sigma^{2}(t)$. The converse is a famous theorem due to P. Lévy and known as characterization of P. Lévy of the Wiener process. We now formulate it and refer to Jacod \& Shiryaev (2000), Theorem II.4.4 for the proof.

1.2.9 Theorem (Characterization of P. Lévy of the Wiener process). Let $W$ be a continuous $\mathbb{F}$-local martingale such that $W_{0}=0$. Then it is a Wiener process relative to $\mathbb{F}$ if $\langle W, W\rangle$ is deterministic, say $\langle W, W\rangle_{t}=\sigma^{2}(t)$, for some increasing continuous function $\sigma^{2}(\cdot)$. In this case the function $\sigma^{2}(\cdot)$ is the variance function of the Wiener process $W$.

In Jacod \& Shiryaev (2000), Theorem II.4.4, it is proven that if $W$ is a Wiener process relative to $\mathbb{F}$ with variance function $\sigma^{2}(\cdot)$, then the random variable $W_{t}-W_{s}$ is Gaussian, centered and with variance $\sigma^{2}(t)-\sigma^{2}(s)$, for all $0 \leq s \leq t$. Therefore the characteristic function of $W_{t}-W_{s}$ is given by

$$
\mathbb{E}\left[\mathrm{e}^{i u\left(W_{t}-W_{s}\right)}\right]=\mathrm{e}^{-\frac{1}{2} u^{2}\left(\sigma^{2}(t)-\sigma^{2}(s)\right)}, \quad 0 \leq s \leq t, \quad u \in \mathbb{R} .
$$

\subsubsection{Orthogonality of Local Martingales}

We recall that two local martingales $M$ and $N$ are called orthogonal if $M N \in \mathscr{M}_{\text {loc }}$. We denote it by $M \perp N$. If $\mathscr{X} \subseteq \mathscr{M}_{\text {loc }}$ and $M \in \mathscr{M}_{\text {loc }}$ is such that $M \perp N$ for every $N \in \mathscr{X}$, we say that $M$ is orthogonal to $\mathscr{X}$ and denote it by $M \perp \mathscr{X}$. This notation is ambiguous with the notation introduced to denote the orthogonality of two functions in $L^{2}(\mathbb{P})$. However, in most of cases, it will be clear from the contest to which orthogonality we refer. When it will not be possible, we shall prefer the terminology strong orthogonality to designate the notion of orthogonality for local martingales. Let us assume that $M$ and $N$ belong to $\mathscr{H}^{2}$ and that $M_{0}=N_{0}=0$. By the definition of the point brackets associated with $M$ and $N$, it is clear that $M \perp N$ if and only if $\langle M, N\rangle=0$. From (1.7) and (1.4) this means $\mathbb{E}\left[M_{\infty} N_{\infty}\right]=0$. Hence the strong orthogonality of the martingales $M$ and $N$ implies the orthogonality of the terminal values $M_{\infty}$ and $N_{\infty}$ in $L^{2}(\mathbb{P})$ and therefore the orthogonality of the martingale $M$ and $N$ in the Hilbert space $\left(\mathscr{H}^{2},\|\cdot\|_{2}^{*}\right)$. For this reason we use the same notation to designate the orthogonality of local martingales and the orthogonality in $L^{2}(\mathbb{P})$. The converse is not true however (for a counterexample cf., e.g., Protter (2005), IV.§3, the comment after the last definition on page 181) and this explains the reason of the terminology "strong orthogonality".

The notion of orthogonality for local martingales leads to the one of purely discontinuous local martingale: A local martingale $M$ is called purely discontinuous if $M_{0}=0$ and if it is orthogonal to every continuous local martingale. We denote by $\mathscr{M}_{\text {loc }}^{c}$ and $\mathscr{M}_{\text {loc }}^{d}$ the classes of continuous and purely discontinuous local martingales, respectively. In the next proposition we collect some relevant properties of orthogonal local martingales.

1.2.10 Proposition. (i) If $M$ and $N$ belong to $\mathscr{H}^{2}$ and $M$ is orthogonal to $N$, then the local martingale $M N$ is such that $\sup _{t \geq 0}\left|M_{t} N_{t}\right|<+\infty$. In particular, $M N$ is a uniformly integrable martingale. 
(ii) A local martingale $M$ is orthogonal to itself if and only if $M_{0} \in L^{2}(\mathbb{P})$ and $M=M_{0}$ a.s.

(iii) If $M$ and $N$ are orthogonal local martingales, then the local martingales $M^{T}$ and $N^{S}$ are again orthogonal, for every pair of stopping times $T$ and $S$.

Proof. For (i) cf. Jacod (1979), Proposition 2.11 while for (ii) and (iii) we refer to Jacod \& Shiryaev (2000), Lemma I.4.13.

A deep result of martingales theory asserts that any local martingale $M$ admits the a.s. unique decomposition

$$
M=M_{0}+M^{c}+M^{d},
$$

where $M_{0}^{c}=M_{0}^{d}=0, M^{c}$ is a continuous local martingale and $M^{d}$ is a purely discontinuous local martingale (cf. Jacod \& Shiryaev (2000), Theorem I.4.18). We call $M^{c}$ and $M^{d}$ as in (1.9) the continuous and the purely-discontinuous part of $M$, respectively.

\subsubsection{Semimartingales}

An adapted process $X$ is called a semimartingale if

$$
X=X_{0}+M+A,
$$

where $X_{0}$ is an $\mathscr{F}_{0}$-measurable random variable, $M \in \mathscr{M}_{\text {loc }, 0}$ and $A \in \mathscr{V}$. We call $M$ the martingale part and $A$ the finite-variation part of the semimartingale $X$, respectively. We call a representation as (1.10) a semimartingale decomposition of $X$. Note that the paths of a semimartingale are càdlàg (we assume that martingales are càdlàg if not otherwise specified). Clearly $\mathscr{M}_{\text {loc }}$ and $\mathscr{V}$ are classes of semimartingales. From (1.9) we can rewrite (1.10) as

$$
X=X_{0}+M^{c}+M^{d}+A
$$

Although the decomposition (1.11) is, in general, not unique, the martingale $M^{c}$ is uniquely determined (up to indistinguishability) by $X$. In other words, if $X$ is a semimartingale and $(M, A)$ and $\left(M^{\prime}, A^{\prime}\right)$ are two semimartingale decompositions of $X$, then $M^{c}$ and $M^{\prime c}$ are indistinguishable (cf. Jacod \& Shiryaev (2000), Proposition I.4.21). In the sequel, we shall denote by $X^{c}$ the unique continuous local martingale meeting (1.11) and call it the continuous martingale part of $X$. Let $A$ and $M$ be a semimartingale decomposition of $X$. We notice that from (1.10) the sum $\sum_{0<s \leq t}\left(\Delta X_{s}\right)^{2}$ is a.s. finite, $t \geq 0$. Indeed, $\sum_{0<s \leq t}\left|\Delta A_{s}\right| \leq \operatorname{Var}(A)_{t}<+\infty, t \geq 0$ (we recall that $A$ is càdlàg and so the previous summation has at most countably many summands). This implies that also $\sum_{0<s \leq t}\left(\Delta A_{s}\right)^{2}<$ is finite. Furthermore, He, Wang \& Yan (1992), Lemma 7.27 ensures that $\sum_{0<s \leq t}\left(\Delta M_{s}\right)^{2}<+\infty$ a.s., $t \geq 0$. Hence for any semimartingale $X$ we can introduce the process $[X, X]=\left([X, X]_{t}\right)_{t \geq 0}$ by

$$
[X, X]_{t}:=\left\langle X^{c}, X^{c}\right\rangle_{t}+\sum_{0<s \leq t}\left(\Delta X_{s}\right)^{2}, \quad t \geq 0
$$


Clearly, the process $[X, X]$ belongs to $\mathscr{V}^{+}$. Moreover, it is continuous if and only if $X$ is continuous (notice that from Jacod \& Shiryaev (2000), Theorem I.4.2 the point brackets of a continuous local martingale are continuous).

If $X$ and $Y$ are two semimartingales and $X^{c}$ and $Y^{c}$ are the respective continuous martingale parts, we define the process $[X, Y]=\left([X, Y]_{t}\right)_{t \geq 0}$ by

$$
[X, Y]_{t}=\frac{1}{4}\left([X+Y, X+Y]_{t}-[X-Y, X-Y]_{t}\right), \quad t \geq 0 .
$$

Thanks to Jacod \& Shiryaev (2000), Proposition I.3.3, $[X, Y]$ belongs to $\mathscr{V}$. Obviously, the following identity holds:

$$
[X, Y]_{t}:=\left\langle X^{c}, Y^{c}\right\rangle_{t}+\sum_{0 \leq s \leq t} \Delta X_{s} \Delta Y_{s}, \quad t \geq 0
$$

We call the process $[X, Y]$ the quadratic covariation or simply the covariation of $X$ and $Y$. If $X$ or $Y$ are continuous, then $[X, Y]=\left\langle X^{c}, Y^{c}\right\rangle$ and $[X, Y]$ is continuous (cf. Jacod \& Shiryaev (2000), Theorem I.4.2). From (1.12), it is immediate to see that $\Delta[X, Y]=\Delta X \Delta Y$. Moreover, if $X$ is continuous and $Y$ is of finite variation, then $[X, Y]=0$. Indeed, if $X$ is continuous, from (1.12) we have $[X, Y]=\left\langle X^{c}, Y^{c}\right\rangle$. On the other side, a semimartingale decomposition of $Y$ is $Y=0+Y$. But the continuous martingale part $Y^{c}$ of $Y$ is uniquely determined and $Y^{c}=0^{c}=0$. The next relation is immediate from (1.6) and (1.12). It explains the behavior of the quadratic covariation process with respect to the operation of stopping. For any stopping time $T$ it follows:

$$
\left[X, Y^{T}\right]=\left[X^{T}, Y\right]=\left[X^{T}, Y^{T}\right]=[X, Y]^{T} .
$$

If $M$ and $N$ are local martingales, the process $[M, N]$ has additional properties as the following proposition shows:

1.2.11 Proposition. (i) If $M$ and $N$ belong to $\mathscr{M}_{\mathrm{loc}, 0}$, then $M N-[M, N]$ is a local martingale and $M \perp N$ if and only if $[M, N] \in \mathscr{M}_{\mathrm{loc}}$.

(ii) If $M$ and $N$ belong to $\mathscr{H}_{\mathrm{loc}}^{2}$, then $[M, N] \in \mathscr{A}_{\mathrm{loc}}$ and $\langle M, N\rangle$ is its compensator, i.e., $\langle M, N\rangle \in \mathscr{A}_{\mathrm{loc}}$ and $[M, N]-\langle M, N\rangle \in \mathscr{M}_{\mathrm{loc}, 0}$. In case $M, N \in \mathscr{H}^{2}$ we have $[M, N] \in \mathscr{A}$ and furthermore $\sup _{t \geq 0}\left|M_{t} N_{t}-[M, N]_{t}\right|, \sup _{t \geq 0}\left|[M, N]_{t}-\langle M, N\rangle_{t}\right| \in$ $L^{1}(\mathbb{P})$. In particular, $M N-[M, N] \in \mathscr{M}$ and $[M, N]-\langle M, N\rangle \in \mathscr{M}_{0}$.

Proof. For (i) cf. Jacod (1979), Corollary 2.29. Now we come to (ii). We assume $M, N \in \mathscr{H}^{2}$ and the other part follows by localization. In case $M, N \in \mathscr{H}^{2}$, to see $[M, N] \in \mathscr{A}$ and $\sup _{t>0}\left|M_{t} N_{t}-[M, N]_{t}\right| \in L^{1}(\mathbb{P})$ cf. Jacod (1979), Lemma 2.28. From (i) $M N-[M, N]$ is a local martingale and because $\sup _{t>0}\left|M_{t} N_{t}-[M, N]_{t}\right| \in$ $L^{1}(\mathbb{P})$ it is dominated in $L^{1}(\mathbb{P})$ and therefore it is a uniformly integrable martingale. The process $\langle M, N\rangle$ is defined and $M N-\langle M, N\rangle$ belongs to $\mathscr{M}$ (cf. Jacod \& Shiryaev (2000), Theorem I.4.2) Subtracting the latter process to $M N-[M, N]$ we obtain $[M, N]-\langle M, N\rangle \in \mathscr{M}$. By definition we have $[M, N]_{0}=\langle M, N\rangle_{0}=0$ and the proof is finished. 


\subsection{Spaces of Martingales and Stochastic Integration}

In this section we introduce some important subspaces of uniformly integrable martingales and briefly recall the definition of stochastic integral for an arbitrary local martingale: Given a local martingale $M$, we define the largest class of processes such that the stochastic integral with respect to $M$ is again a local martingale. We also define the stochastic integral with respect to a semimartingale $X$. However, we do not consider the largest class of processes such that the stochastic integral with respect to $X$ is again a semimartingale. Instead, we restrict our attention to a particular case. Finally, we introduce Itô's formula.

\subsubsection{Spaces of Martingales}

In $\S 1.2 .5$ we have introduced the space $\mathscr{H}^{2}$ of square integrable martingales. Now we want to generalize this definition to the one of $q$-integrable martingales. Let $M \in \mathscr{M}_{\text {loc }}$ and $q \in[1,+\infty]$. We put

$$
\|M\|_{\mathscr{H}^{q}}:=\left\|\sup _{t \geq 0}\left|M_{t}\right|\right\|_{q},
$$

where $\|\cdot\|_{q}$ denotes the $L^{q}(\mathbb{P})$-norm, and introduce the following classes of local martingales:

$$
\mathscr{H}^{q}:=\left\{M \in \mathscr{M}_{\text {loc }}:\|M\|_{\mathscr{H}^{q}}<+\infty\right\} .
$$

We denote by $\mathscr{H}_{0}^{q}$ the subset of elements of $\mathscr{H}^{q}$ starting at 0 . The spaces $\mathscr{H}_{\text {loc }}^{q}$ and $\mathscr{H}_{\text {loc, } 0}^{q}$ are introduced in the usual way from $\mathscr{H}^{q}$ and $\mathscr{H}_{0}^{q}$, respectively. The space $\left(\mathscr{H}^{q},\|\cdot\|_{\mathscr{H}^{q}}\right)$ is a Banach space. If $p, q \in[1,+\infty], p \leq q$, then $\mathscr{H}^{q} \subseteq \mathscr{H}^{p}$ and $\mathscr{H}^{q}$ is dense in $\left(\mathscr{H}^{p},\|\cdot\|_{\mathscr{H}^{p}}\right)$ (cf. Jacod (1979), Proposition 2.39). We observe that the space of local martingales $\mathscr{H}^{q}$ is in fact a subspace of $\mathscr{M}$. Indeed, if $M \in \mathscr{H}^{q}$, then it is a local martingale which is dominate in $L^{1}(\mathbb{P})$ by $\sup _{t \geq 0}\left|M_{t}\right|^{q}$. Therefore the terminal value $M_{\infty}$ of $M \in \mathscr{H}^{q}$ exists and belongs to $L^{q}(\mathbb{P})$. For any $M \in \mathscr{H}^{q}, q \geq 1$, the process $|M|^{q}$ is a uniformly integrable submartingale and obviously $\sup _{t \geq 0} \mathbb{E}\left[\left|M_{t}\right|^{q}\right] \leq \mathbb{E}\left[\sup _{t \geq 0}\left|M_{t}\right|^{q}\right]<+\infty$. Now we state Doob's inequality (cf. He, Wang \& Yan (1992), Theorem 2.49). If $q>1$ and $X$ is a nonnegative càdlàg submartingale such that $\sup _{t \geq 0} \mathbb{E}\left[X_{t}^{q}\right]<+\infty$, then $\sup _{t \geq 0} X_{t} \in L^{q}(\mathbb{P})$ and

$$
\mathbb{E}\left[\sup _{t \geq 0} X_{t}^{q}\right] \leq\left(\frac{q}{q-1}\right)^{q} \sup _{t \geq 0} \mathbb{E}\left[X_{t}^{q}\right]=\mathbb{E}\left[X_{\infty}^{q}\right]
$$

We assume $q>1$ and introduce the norm

$$
\|M\|_{q}^{*}:=\left\|M_{\infty}\right\|_{q}
$$

Obviously, $\mathbb{E}\left[\left|M_{\infty}\right|^{q}\right] \leq \mathbb{E}\left[\sup _{t \geq 0}\left|M_{t}\right|^{q}\right]<+\infty$ and so (1.16) yields the equivalence of the norms $\|\cdot\|_{\mathscr{H}^{q}}$ and $\|\cdot\|_{q}^{*}$. The norm $\|\cdot\|_{q}^{*}$ is specially important because, for $q>1$, the space $\left(\mathscr{H}^{q},\|\cdot\|_{q}^{*}\right)$ can be identified with the space $\left(L^{q}(\mathbb{P}),\|\cdot\|_{q}\right)$ by identifying each martingale $M \in \mathscr{H}^{q}$ with its terminal value $M_{\infty} \in L^{q}(\mathbb{P})$. It is important to stress that the space $\mathscr{H}^{2}$ equipped with the norm $\|\cdot\|_{2}^{*}$ is a Hilbert space because it is isomorphic to $\left(L^{2}(\mathbb{P}),\|\cdot\|_{2}\right)$. The space $\mathscr{H}^{2}$ equipped with the norm $\|\cdot\|_{\mathscr{H}^{2}}$ is not a Hilbert space however, because the previous identification 
cannot be done. Because of the identification of $\left(\mathscr{H}^{q},\|\cdot\|_{q}^{*}\right)$ with $\left(L^{q}(\mathbb{P}),\|\cdot\|_{q}\right)$ for $q>1$, we can identify the dual of $\left(\mathscr{H}^{q},\|\cdot\|_{q}^{*}\right)$ with $\left(\mathscr{H}^{p},\|\cdot\|_{p}^{*}\right)$, where $p$ is the conjugate exponent of $q$, i.e., $p \in(1,+\infty)$ is such that $\frac{1}{p}+\frac{1}{q}=1$. The same is not true for $q=1$. Indeed, for $q=1$ Doob's inequality does not hold in the form of (1.16). On the other side, we cannot identify the space $\mathscr{H}^{1}$ with $L^{1}(\mathbb{P})$ : From Jacod \& Shiryaev (2000), Theorem I.1.42, is the space $\mathscr{M}$ of uniformly integrable martingales that can be identified in a natural way with $L^{1}(\mathbb{P})$ and, in general, $\mathscr{H}^{1} \subsetneq \mathscr{M}$. In the sequel the spaces $\left(\mathscr{H}_{0}^{q},\|\cdot\|_{\mathscr{H}^{q}}\right)$ will play a key role. In particular, we shall introduce the dual space of $\mathscr{H}_{0}^{1}$.

Let $M$ be a uniformly integrable martingale. We introduce the norm

$$
\|M\|_{B M O}:=\sup _{T}\left\|M_{\infty}-M_{T-}\right\| / \sqrt{\mathbb{P}(T<+\infty)},
$$

where the supremum is taken over all stopping times with the conventions $M_{\infty-}:=$ $M_{\infty}$ and $0 / 0:=0$. By $B M O$ we denote space of uniformly integrable martingales with finite $B M O$-norm, that is,

$$
B M O:=\left\{M \in \mathscr{M}:\|M\|_{B M O}<+\infty\right\} .
$$

By $B M O_{0}$ we denote the subset of elements of $B M O$ starting at zero, while $B M O_{\text {loc }}$ and $B M O_{\mathrm{loc}, 0}$ are introduced as usual from $B M O$ and $B M O_{0}$, respectively, by localization. The following inequalities and inclusions hold (cf. Jacod (1979), p. 28, formula (2.8) and (2.9)).

$$
\|M\|_{\mathscr{H}^{2}} \leq 2\|M\|_{B M O} \leq 4\|M\|_{\mathscr{H}^{\infty}}, \quad \mathscr{H}^{\infty} \subseteq B M O \subseteq \mathscr{H}^{2}
$$

Furthermore, $\mathscr{H}_{\mathrm{loc}}^{1}=\mathscr{M}_{\mathrm{loc}}$ and $B M O_{\mathrm{loc}}=\mathscr{H}_{\mathrm{loc}}^{\infty}$. In particular, $B M O \subseteq \mathscr{H}_{\mathrm{loc}}^{\infty}(\mathrm{cf}$. Jacod (1979), Lemma 2.38). We recall that $\mathscr{M}_{\mathrm{loc}}^{c}$ and $\mathscr{M}_{\mathrm{loc}}^{d}$ designate the spaces of continuous and purely discontinuous local martingales, respectively, and introduce the following notation:

$$
\mathscr{H}^{q, c}:=\mathscr{H}^{q} \cap \mathscr{M}_{\mathrm{loc}}^{c} ; \quad \mathscr{H}^{q, d}:=\mathscr{H}^{q} \cap \mathscr{M}_{\mathrm{loc}}^{d} .
$$

For every $q \in[1,+\infty]$ the subspaces $\mathscr{H}_{0}^{q}, \mathscr{H}^{q, c}$ and $\mathscr{H}^{q, d}$ are closed in $\left(\mathscr{H}^{q},\|\cdot\|_{\mathscr{H}^{q}}\right)$ (cf. Jacod (1979), Lemma 2.12). Therefore, because the norm $\|\cdot\|_{2}^{*}$ is equivalent to the norm $\|\cdot\|_{\mathscr{H}^{2}}$, the space $\mathscr{H}_{0}^{2}$ is closed in $\left(\mathscr{H}^{2},\|\cdot\|_{2}^{*}\right)$ and so $\left(\mathscr{H}_{0}^{2},\|\cdot\|_{2}^{*}\right)$ is a Hilbert space.

Now we state some fundamental inequalities. The first is Kunita-Watanabe's inequality (cf. Jacod (1979), Theorem 2.32): Let $q \in(1,+\infty) ; M, N \in \mathscr{M}_{\text {loc }}$; be $p$ the conjugate exponent of $p$ and $U$ an optional process. Then

$$
\mathbb{E}\left[|U| \cdot \operatorname{Var}([M, N])_{\infty}\right] \leq\left\|\left(U^{2} \cdot[M, M]_{\infty}\right)^{1 / 2}\right\|_{q}\left\|[N, N]_{\infty}^{1 / 2}\right\|_{p} .
$$

Kunita-Watanabe's inequality holds only for $q$-integrable martingales with $q>1$. The following inequality, known as Fefferman's inequality, generalize (1.21) to the case $q=1$. We refer to Jacod (1979), Theorem 2.33. For any $M, N \in \mathscr{M}$ and $U$ optional process, we have

$$
\mathbb{E}\left[|U| \cdot \operatorname{Var}([M, N])_{\infty}\right] \leq\left\|\left(U^{2} \cdot[M, M]_{\infty}\right)^{1 / 2}\right\|_{1}\|N\|_{B M O}
$$


The last of the inequalities that we need is Davis-Burkholder-Gundy's inequality which we abbreviate by $D B G$ 's inequality (cf. Jacod (1979), Theorem 2.34): Let $M \in \mathscr{M}_{\text {loc }}$ and $q \in[1,+\infty)$. There exist two positive constants $c_{q}$ and $C_{q}$ such that

$$
\|M\|_{\mathscr{H}^{q}} \leq c_{q}\left\|[M, M]_{\infty}^{1 / 2}\right\|_{q} \leq C_{q}\|M\|_{\mathscr{H}^{q}} .
$$

Formula (1.23) expresses that the norms $\|M\|_{\mathscr{H}^{q}}$ and $\left\|[M, M]_{\infty}^{1 / 2}\right\|_{q}$ are equivalent. If $q=2$ and $M \in \mathscr{H}_{0}^{2}$ we get Doob's inequality. Indeed, by Proposition 1.2.11, $M^{2}-[M, M] \in \mathscr{H}_{0}^{1}$ and so $\mathbb{E}\left[[M, M]_{\infty}\right]=\mathbb{E}\left[M_{\infty}^{2}\right]=\sup _{t \geq 0} \mathbb{E}\left[M_{t}^{2}\right]$

A consequence of Kunita-Watanabe's inequality (resp., of Fefferman's inequality) and DBG's inequality with $U=1$ is that if $M \in \mathscr{H}^{q}, q>1$ (resp. $q=1$ ), and $N \in \mathscr{H}^{p}$, where $p$ is the conjugate exponent of $q$ (resp., $N \in B M O$ ), then the process $[M, N]$ belongs to $\mathscr{A}$, i.e., $[M, N]_{\infty} \in L^{1}(\mathbb{P})$. Therefore if $M \in \mathscr{H}^{q}$, $q>1$ (resp., $q=1$ ), and $N \in \mathscr{H}^{p}$, where $p$ is the conjugate exponent of $q$ (resp., $N \in B M O)$, we can define the functional

$$
C_{N}(M):=\mathbb{E}\left[[M, N]_{\infty}\right], \quad M \in \mathscr{H}^{q}, N \in \mathscr{H}^{p}(\operatorname{resp} ., N \in B M O) .
$$

The following lemma holds:

1.3.1 Lemma. Let $C_{N}(\cdot)$ be the linear functional introduced in (1.24). If $M \in \mathscr{H}^{q}$, $q>1$ (resp., $q=1$ ), and $N \in \mathscr{H}^{p}$, where $p$ is the conjugate exponent of $q$ (resp., $N \in B M O)$, the linear functional $C_{N}(\cdot)$ is continuous on $\mathscr{H}^{q}$.

Proof. We verify only the case $M \in \mathscr{H}^{q}, q>1$ and $N \in \mathscr{H}^{p}$, where $p$ is the conjugate exponent of $q$. The case $q=1$ and $N \in B M O$ can be shown in a similar way and is a consequence of Fefferman's inequality. Because of Kunita-Watanabe's inequality and of DBG's inequality with $U=1$ we have

$$
\mathbb{E}\left[\left|[M, N]_{\infty}\right|\right] \leq\left\|[M, M]_{\infty}^{1 / 2}\right\|_{q}\left\|[N, N]_{\infty}^{1 / 2}\right\|_{p} \leq C_{q} C_{p}\|M\|_{\mathscr{H}^{q}}\|N\|_{\mathscr{H}^{p}}<+\infty
$$

This, in particular, shows that $C_{N}(\cdot)$ is a functional on $\mathscr{H}^{q}$ which is linear and bounded. Therefore it is a continuous functional on $\mathscr{H}^{q}$.

Thanks to Fefferman's inequality and DBG's inequality, we can identify the space $\left(B M O_{0},\|\cdot\|_{B M O}\right)$ with the dual space of $\left(\mathscr{H}_{0}^{1},\|\cdot\|_{\mathscr{H}^{1}}\right)$ in the following way: We identify every $N \in B M O_{0}$ in a unique way with the continuous linear form $C_{N}(\cdot)$ defined by (1.24). We do not show this result and we refer to Protter (2005), Theorem IV.55. We only verify that $C_{N}$ in an injective functional from $B M O_{0}$ into the dual of $\mathscr{H}_{0}^{1}$. Let $N^{\prime}, N \in B M O_{0}$ be such that $C_{N}=C_{N^{\prime}}$ on $\mathscr{H}_{0}^{1}$. Then $C_{N-N^{\prime}}(Z)=0$, for every $Z$ which belongs to $\mathscr{H}_{0}^{1}$. Hence for any stopping time $T$ and any $M \in \mathscr{H}_{0}^{1}$, because of (1.13) and the stability under stopping of $\mathscr{H}_{0}^{1}$, it follows

$$
\mathbb{E}\left[\left[M, N-N^{\prime}\right]_{T}\right]=\mathbb{E}\left[\left[M, N-N^{\prime}\right]_{\infty}^{T}\right]=C_{N-N^{\prime}}\left(M^{T}\right)=0
$$

and so Lemma 1.2.2 implies that $\left[M, N-N^{\prime}\right]$ belongs to $\mathscr{M}_{0}$, for every $M \in \mathscr{H}_{0}^{1}$, i.e., $N-N^{\prime} \perp \mathscr{H}_{0}^{1}$ (cf. Proposition 1.2.11.(i)). This yields $N-N^{\prime}$ is orthogonal to itself because $B M O_{0} \subseteq \mathscr{H}_{0}^{2} \subseteq \mathscr{H}_{0}^{1}$. Proposition 1.2.10.(ii) implies that $N-N^{\prime}=$ $N_{0}-N_{0}^{\prime}=0$ a.s., that is, $N=N^{\prime}$ a.s., proving that $C_{N}(\cdot)$ is injective from $B M O_{0}$ into the dual of $\mathscr{H}_{0}^{1}$. 
We consider $q \in(1,+\infty)$ and its conjugate exponent $p \in(1,+\infty)$. We have already discussed that, thanks to Doob's inequality, the dual space of $\left(\mathscr{H}^{q},\|\cdot\|_{q}^{*}\right)$ can be identified with $\left(\mathscr{H}^{p},\|\cdot\|_{q}^{*}\right)$, where $p$ is the conjugate exponent of $q$ and $q>1$. However, it is not difficult to see that the dual space of $\left(\mathscr{H}_{0}^{q},\|\cdot\|_{\mathscr{H}^{q}}\right)$ can be identified with $\left(\mathscr{H}_{0}^{p},\|\cdot\|_{\mathscr{H}^{p}}\right)$ by means of the functional $C_{N}(\cdot)$ in a similar way as we did for the dual space of $\left(\mathscr{H}_{0}^{1},\|\cdot\|_{\mathscr{H}^{1}}\right)$. We do not go further into details and we refer to Jacod (1979), Remark 2.36. We only stress that this identification cannot be done between the dual space of $\left(\mathscr{H}^{q},\|\cdot\|_{\mathscr{H}^{q}}\right)$ and $\left(\mathscr{H}^{p},\|\cdot\|_{\mathscr{H}^{p}}\right)$ in the same way: If we do not assume $N_{0}=M_{0}=0$, the functional $C_{N}(\cdot)$ is not injective from $\left(\mathscr{H}^{p},\|\cdot\|_{\mathscr{H}^{p}}\right)$ into the dual of $\left(\mathscr{H}^{q},\|\cdot\|_{\mathscr{H}^{q}}\right)$ because we always have $[M, N]_{0}=0$. In Jacod (1979) this problem on the initial value does not appear. The difference is that in Jacod (1979), the convention $X_{0-}:=0$ for the left-hand limit of the initial value of a càdlàg process $X$ is used and from this it follows that $[M, N]_{0}=M_{0} N_{0}$.

\subsubsection{Stochastic Integral for Local Martingales}

In this part we briefly recall the definition of the stochastic integral with respect to a local martingale for the largest class of predictable processes such that the stochastic integral remains a local martingale. Let $M$ be a local martingale and $q \in[1,+\infty)$. For every measurable process $H$ we introduce the norm

$$
\|H\|_{\mathrm{L}^{q}(M)}:=\left\|\left(H^{2} \cdot[M, M]_{\infty}\right)^{1 / 2}\right\|_{q} .
$$

Then we introduce the following class of processes:

$$
\mathrm{L}^{q}(M):=\left\{H \text { predictable process : }\|H\|_{\mathrm{L}^{q}(M)}<+\infty\right\} .
$$

We call $\mathrm{L}^{q}(M)$ the space of integrands of order $q$ for the local martingale $M$. The classes $\mathrm{L}_{\text {loc }}^{q}(M)$ are introduced from $\mathrm{L}^{q}(M)$ in following way: We say that a predictable process $H$ belongs to $\mathrm{L}_{\text {loc }}^{q}(M)$ if there exists an increasing sequence $\left(T_{n}\right)_{n \geq 0}$ of stopping time such that $T_{n} \uparrow+\infty$ and $1_{\left[0, T_{n}\right]} H \in \mathrm{L}^{q}(M)$. Although this procedure is different from the usual localization procedure, we shall use the same terminology and the same notations for both of them. Obviously, $\mathrm{L}^{q}(M) \subseteq \mathrm{L}^{p}(M)$ and $\mathrm{L}_{\text {loc }}^{q}(M) \subseteq \mathrm{L}_{\text {loc }}^{p}(M)$ if $q \geq p$. We shall introduce the stochastic integral with respect to a local martingale $M$ for predictable processes belonging to $\mathrm{L}_{\mathrm{loc}}^{1}(M)$. We follow Jacod (1979), II.§2.

In a first step we introduce the stochastic integral with respect to continuous local martingales. Let $M \in \mathscr{M}_{\mathrm{loc}, 0}^{c}$. In this special case the space $\mathrm{L}_{\mathrm{loc}}^{q}(M)$ coincides with $\mathrm{L}_{\text {loc }}^{1}(M)$ for every $q \geq 1$. The inclusion $\mathrm{L}_{\text {loc }}^{1}(M) \supseteq \mathrm{L}_{\text {loc }}^{q}(M)$ for every $q \geq 1$ is clear. For the converse inclusion we first observe that $[M, M]$ is an increasing process (cf. $\S 1.2 .8)$. If $H \in \mathrm{L}_{\text {loc }}^{1}(M)$, the process $\left(H^{2} \cdot[M, M]\right)^{1 / 2}$ is locally integrable. Hence it assumes finite values at every time $t$ and so also the process $H^{2} \cdot[M, M]$ does. From Jacod \& Shiryaev (2000), Proposition I.3.5, the process $H^{2} \cdot[M, M]$ is an increasing process and furthermore is continuous because $M$ is a continuous local martingale. Therefore $H^{2} \cdot[M, M]$ is locally bounded. In particular, this yields $H \in \mathrm{L}_{\text {loc }}^{q}(M)$ for every $q \geq 1$. Then to introduce the stochastic integral of a process $H \in \mathrm{L}_{\text {loc }}^{1}(M)$ with respect to $M \in \mathscr{M}_{\text {loc }, 0}^{c}$, it is enough, by localization, to define the stochastic integral for $H \in \mathrm{L}^{2}(M)$. 
We consider a continuous local martingale $M \in \mathscr{M}_{\mathrm{loc}, 0}^{c}$ and $H \in \mathrm{L}^{2}(M)$. We define the linear form $C(\cdot)$ by $C(N):=\mathbb{E}\left[H \cdot[M, N]_{\infty}\right]$, which is well-defined for every $N \in \mathscr{H}_{0}^{2}$. Because of Kunita-Watanabe's inequality and of DBG's inequality (cf. (1.21) and (1.23)), we have

$$
\begin{aligned}
|C(N)| & \leq\|H\|_{\mathrm{L}^{2}(M)}\left\|[N, N]_{\infty}^{1 / 2}\right\|_{2} \\
& \leq c_{2}\|H\|_{\mathrm{L}^{2}(M)}\|N\|_{\mathscr{H}^{2}} \\
& \leq c_{2}\|H\|_{\mathrm{L}^{2}(M)}\|N\|_{2}^{*}<+\infty, \quad N \in \mathscr{H}_{0}^{2},
\end{aligned}
$$

where in the last passage we used that the norm $\|\cdot\|_{\mathscr{H}^{2}}$ and the norm $\|\cdot\|_{2}^{*}$ are equivalent, as a consequence of Doob's inequality (cf. (1.16)). Hence $C(\cdot)$ is linear and continuous on $\left(\mathscr{H}_{0}^{2},\|\cdot\|_{2}^{*}\right)$. The space $\left(\mathscr{H}_{0}^{2},\|\cdot\|_{2}^{*}\right)$ is a reflexive Hilbert space and therefore, because of Riesz's Theorem, there exists a unique element $X \in \mathscr{H}_{0}^{2}$ such that the functional $C(\cdot)$ can be represented as

$$
C(N):=\mathbb{E}\left[H \cdot[M, N]_{\infty}\right]=\mathbb{E}\left[X_{\infty} N_{\infty}\right]=:(X, N)_{\mathscr{H}_{0}^{2}}, \quad N \in \mathscr{H}_{0}^{2},
$$

where $(\cdot, \cdot)_{\mathscr{H}_{0}^{2}}$ denotes the scalar product in $\left(\mathscr{H}_{0}^{2},\|\cdot\|_{2}^{*}\right)$. We give the following definition.

1.3.2 Definition. Let $M \in \mathscr{M}_{\mathrm{loc}, 0}^{c}$ and $H \in \mathrm{L}^{2}(M)$. We call the unique element $X$ satisfying (1.27) the stochastic integral of $H$ with respect to $M$ and we denote it by $H \cdot M:=X$ or by $\int_{0}^{\cdot} H_{s} \mathrm{~d} M_{s}:=X$.

We notice that Definition 1.3.2 does not introduce any ambiguity with the notation $K \cdot A$ or $\int_{0}^{\cdot} K_{s} \mathrm{~d} A_{s}$ used to denote the stochastic integral of a measurable process $K$ with respect to $A \in \mathscr{V}$ because if $M \in \mathscr{M}_{\mathrm{loc}}^{c} \cap \mathscr{V}$, then $M \equiv 0$ : It is well known that any local martingale which belongs to $\mathscr{V}$ and is continuous is evanescent (cf. Jacod \& Shiryaev (2000), Lemma I.4.13 and I.4.14).

In Jacod (1979), Proposition 2.43, the following characterization of the stochastic integral with respect to continuous local martingales is established: Let $M \in \mathscr{M}_{\mathrm{loc}, 0}^{c}$ and $H \in \mathrm{L}^{2}(M)$. Then $H \cdot M \in \mathscr{H}_{0}^{2, c}$ and it is the unique element of $\mathscr{H}_{0}^{2}$ such that

$$
[H \cdot M, N]=H \cdot[M, N], \quad N \in \mathscr{H}_{0}^{2} .
$$

From Jacod (1979), formula (2.44), we have that, for every stopping time $T$,

$$
(H \cdot M)^{T}=H \cdot M^{T}=\left(1_{[0, T]} H\right) \cdot M .
$$

More generally, if $M \in \mathscr{M}_{\mathrm{loc}, 0}^{c}$ and $H$ and $K$ are locally bounded predictable processes, then $H \cdot(K \cdot M)=H K \cdot M$. Indeed, by (1.29) this is true if $H=1_{[0, T]}$, where $T$ is a stopping time. To obtain the formula for a (locally) bounded predictable $H$ is now a standard procedure with the help of a monotone class argument (cf. §1.1.2).

We notice that for every $N \in \mathscr{H}_{0}^{2}$, because of the continuity of $H \cdot M$ for $M \in$ $\mathscr{M}_{\mathrm{loc}, 0}^{c}$ and $H \in \mathrm{L}^{2}(M)$, the relation $[H \cdot M, N]=\langle H \cdot M, N\rangle$ holds (cf. $\S 1.2 .8$ ). It is clear that the stochastic integral is linear in both the integrator and the integrand, i.e., if $M, N \in \mathscr{M}_{\mathrm{loc}, 0}^{c}$ and $H \in \mathrm{L}^{2}(M) \cap \mathrm{L}^{2}(N)$, then $H \cdot(M+N)=H \cdot M+H \cdot N$, and if $K \in \mathrm{L}^{2}(M)$, then $(H+K) \cdot M=H \cdot M+K \cdot M$. 
We now introduce the stochastic integral with respect to general local martingales for predictable processes in $H \in \mathrm{L}_{\text {loc }}^{1}(M)$. We do not go into details but we refer to Jacod (1979) II. $\S 2$ for a complete and formal treatment of the topic. We recall that any local martingale $M$ can be decomposed in an a.s. unique way as in (1.9) in its continuous and purely discontinuous parts $M^{c}$ and $M^{d}$, respectively.

1.3.3 Definition. Let $M \in \mathscr{M}_{\mathrm{loc}, 0}$ and $H \in \mathrm{L}_{\mathrm{loc}}^{1}(M)$. The stochastic integral of $H$ with respect to $M$ is the unique element $X \in \mathscr{M}_{\text {loc }, 0}$ such that

$$
X^{c}=H \cdot M^{c} \quad \text { and } \quad \Delta X=H \Delta M,
$$

where $M^{c}$ denotes the continuous part of the martingale $M$. We use the notation $H \cdot M:=X$ or $\int_{0}^{\cdot} H_{s} \mathrm{~d} M_{s}:=X$ to denote the unique element $X \in \mathscr{M}_{\mathrm{loc}, 0}$ satisfying $(1.30)$.

Thanks to Lepingle (1977), if $M \in \mathscr{M}_{\text {loc }, 0}$ and $H \in \mathrm{L}_{\text {loc }}^{1}(M)$, the element $X \in$ $\mathscr{M}_{\mathrm{loc}, 0}$ satisfying the properties of Definition 1.3.3 always exists and is unique. We stress that by definition, $H \cdot M_{0}=0$.

If $M \in \mathscr{M}_{\text {loc }} \cap \mathscr{V}$ and $H \in \mathrm{L}_{\text {loc }}^{1}(M)$, then we can also introduce the integral of Stiltjes-Lebesgue of $H$ with respect to $M$. As proved in Jacod (1979), Remark 2.47, in this case the stochastic integral and the integral of Stiltjes-Lebesgue of $H$ with respect to $M$ coincide. Therefore it is not an abuse of notation to designate the stochastic integral with respect to $M \in \mathscr{M}_{\mathrm{loc}, 0}$ in the same way as we did for the integral with respect to processes in $\mathscr{V}$. The following proposition collects some important properties of the stochastic integral. For the proof, cf. Jacod (1979), Proposition 2.48 .

1.3.4 Proposition. Let $M \in \mathscr{M}_{\mathrm{loc}, 0}$ and $H \in \mathrm{L}_{\mathrm{loc}}^{1}(M)$.

(i) $H \cdot M$ is the unique element of $\mathscr{M}_{\mathrm{loc}, 0}$ satisfying $[H \cdot M, N]=H \cdot[M, N]$, for every $N \in \mathscr{M}_{\text {loc }, 0}$.

(ii) $H \cdot M \in \mathscr{H}_{0}^{q}$ (resp., $\mathscr{H}_{\mathrm{loc}, 0}^{q}$ ) if and only if $H \in \mathrm{L}^{q}(M)$ (resp., $\mathrm{L}_{\mathrm{loc}}^{q}(M)$ ).

Sometimes one takes the characterization in Proposition 1.3.4.(i) as the definition of the stochastic integral with respect to a local martingale (cf. Jacod (1979), Remark 2.49 ). Notice that any predictable and locally bounded process belongs to $\mathrm{L}_{\text {loc }}^{1}(M)$. Moreover, for every stopping time $T$ and $H \in \mathrm{L}_{\text {loc }}^{1}(M)$ we have

$$
(H \cdot M)^{T}=H \cdot M^{T}=H 1_{[0, T]} \cdot M
$$

More generally, if $H$ and $K$ are locally bounded and $M \in \mathscr{M}_{\mathrm{loc}, 0}$, then

$$
H \cdot(K \cdot M)=H K \cdot M .
$$

To verify (1.32) is immediate. Indeed, because (1.32) holds for continuous local martingales, we have $(H \cdot(K \cdot M))^{c}=H \cdot(K \cdot M)^{c}=H \cdot\left(K \cdot M^{c}\right)=H K \cdot M^{c}$ and $\Delta(H \cdot(K \cdot M))=H K \Delta M$. The assertion follows from Definition 1.3.3.

The class $\mathrm{L}_{\text {loc }}^{1}(M)$ is the largest class of predictable integrands for the local martingale $M$ such that the stochastic integral is again an element of $\mathscr{M}_{\text {loc }, 0}$. Indeed, Proposition 1.3.4.(i) implies that $H \cdot M \in \mathscr{H}_{\mathrm{loc}, 0}^{1}$ if and only if $H \in \mathrm{L}_{\mathrm{loc}}^{1}(M)$. On the 
other side, we know that $\mathscr{H}_{\mathrm{loc}, 0}^{1}=\mathscr{M}_{\mathrm{loc}, 0}$ (cf. Jacod (1979), Lemma 2.38) and this proves the assertion.

It is clear that the stochastic integral is linear in both the integrand and the integrator, i.e., if $M, N \in \mathscr{M}_{\mathrm{loc}, 0}$ and $H \in \mathrm{L}^{2}(M) \cap \mathrm{L}^{2}(N)$, then $H \cdot(M+N)=$ $H \cdot M+H \cdot N$, and if $K \in \mathrm{L}^{2}(M)$, then $(H+K) \cdot M=H \cdot M+K \cdot N$.

In some literature the stochastic integral with respect to a local martingale $M \in$ $\mathscr{H}_{\text {loc }, 0}^{2}$ is introduced for the class of predictable processes $H$ satisfying the following condition: $\mathbb{E}\left[H^{2} \cdot\langle M, M\rangle_{\infty}\right]<+\infty$ (cf. Jacod \& Shiryaev (2000), I. $\left.\S 4\right)$. However, this class coincides with $\mathrm{L}^{2}(M)$ because $\mathbb{E}\left[H^{2} \cdot[M, M]_{\infty}\right]=\mathbb{E}\left[H^{2} \cdot\langle M, M\rangle_{\infty}\right]$. Indeed, for $M \in \mathscr{H}_{\mathrm{loc}, 0}^{2},[M, M]$ belongs to $\mathscr{A}_{\mathrm{loc}}^{+}$and $\langle M, M\rangle$ is its compensator (cf. Proposition 1.2.11.(ii) and $\S 1.2 .3$ ).

We conclude this part with an analogue of the theorem of Lebesgue on dominated convergence for stochastic integrals. For the proof we refer to Jacod (1979), Proposition 2.73 .

1.3.5 Proposition. Let $M \in \mathscr{M}_{\mathrm{loc}} ; q \in[1,+\infty)$ and $\left(H^{n}\right)_{n \in \mathbb{N}}$ be a sequence of predictable processes converging pointwise to a limit $H$. If there exists a $K \in \mathrm{L}^{q}(M)$ such that $\left|H^{n}\right| \leq K$ for every $n$, then $H^{n} \in \mathrm{L}^{q}(M), H \in \mathrm{L}^{q}(M)$ and $H^{n} \cdot M$ converges in $\left(\mathscr{H}_{0}^{q},\|\cdot\|_{\mathscr{H}^{q}}\right)$ to $H \cdot M$.

\subsubsection{Stochastic Integral for Semimartingales}

In this part we present the stochastic integral with respect to a general semimartingale $X$. For local martingales we have introduced the largest class of predictable processes with respect to which the stochastic integral is again a local martingale. Now we restrict our attention to some special cases of predictable integrands for which the stochastic integral is again a semimartingale without considering the problem of which is the largest class of predictable integrands preserving this property. For a complete description of the topic we suggest Jacod (1979), Chapter II.

Let $\mathrm{K}$ be the following class of predictable processes:

$$
\mathrm{K}:=\left\{H \text { predictable }: 1_{(0,+\infty)} H \text { is locally bounded }\right\} .
$$

We notice that $H \in \mathrm{K}$ is locally bounded if and only if $H_{0}$ is bounded. To introduce the stochastic integral with respect to a semimartingale $X$ of a process in $\mathrm{K}$, we put together what we recalled in $\S 1.2 .3$ and in $\S 1.3 .2$, for the integral with respect to processes of finite variation and with respect to local martingales, respectively.

Let $X$ be a semimartingale and $X=X_{0}+M+A$ be a semimartingale decomposition of $X$, where $M \in \mathscr{M}_{\mathrm{loc}, 0}$ and $A \in \mathscr{V}$. If $H \in \mathrm{K}$, then $H \in \mathrm{L}_{\mathrm{loc}}^{1}(M)$. Furthermore, $H \cdot A$ is well defined as Stiltjes-Lebesgue integral, finite valued (because $A_{0}=0$ ) and it belongs to $\mathscr{V}$ (cf. Jacod \& Shiryaev (2000), Proposition I.3.5). We put

$$
H \cdot X:=H \cdot M+H \cdot A \text {. }
$$

We need to show that the previous definition does not depend on the particular semimartingale decomposition of $X$. Let $A^{\prime} \in \mathscr{V}$ and $M^{\prime} \in \mathscr{M}_{\mathrm{loc}, 0}$ be another semimartingale decomposition of $X$ and $H \in \mathrm{K}$. All the integrals $H \cdot M, H \cdot M^{\prime}$, $H \cdot A$ and $H \cdot A^{\prime}$ are well defined. Moreover $H \cdot A, H \cdot A^{\prime} \in \mathscr{V}$ and $H \cdot M, H \cdot M^{\prime} \in \mathscr{M}_{\text {loc }, 0}$. 
From the identity $M-M^{\prime}=A^{\prime}-A \in \mathscr{M}_{\mathrm{loc}} \cap \mathscr{V}$, we also get $H \cdot\left(M-M^{\prime}\right)=H \cdot\left(A^{\prime}-A\right)$. Indeed, $M-M^{\prime} \in \mathscr{M}_{\mathrm{loc}} \cap \mathscr{V}$ and in this case the stochastic integral and the StiltjesLebesgue integral of $H$ with respect to $\left(M-M^{\prime}\right)$ coincide. By linearity we get $H \cdot M+H \cdot A=H \cdot M^{\prime}+H \cdot A^{\prime}$. Hence formula (1.34) defines the stochastic integral for processes in $\mathrm{K}$ with respect to the semimartingale $X$ independently of the semimartingale decomposition of $X$.

1.3.6 Proposition. Let $X$ be a semimartingale and $H \in \mathrm{K}$. Then $H \cdot X$ is again a semimartingale and (1.34) is a semimartingale decomposition; $(H \cdot X)^{c}=H \cdot X^{c}$; $\Delta(H \cdot X)=H \Delta X$ and $H \cdot X$ is linear in both the integrand and the integrator. Moreover, if $K \in \mathrm{K}$, then $H \cdot(K \cdot X)=H K \cdot X$. For every semimartingale $Y$, we have $[H \cdot X, Y]=H \cdot[X, Y]$.

Proof. cf. Jacod (1979), Proposition 2.51.

Now we are ready to state Itô's formula. Recall that for any càdlàg process $X$ the process $X_{-}$belongs to K. We refer to Jacod (1979), Theorem 2.52.

1.3.7 Theorem. Let $X^{1}, \ldots, X^{d}$ be semimartingales and $f$ a two-times continuously differentiable function from $\mathbb{R}^{d}$ into $\mathbb{R}$. The process $f(X)=\left(f\left(X_{t}\right)\right)_{t \geq 0}$ is again a semimartingale and

$$
\begin{aligned}
f\left(X_{t}\right)=f\left(X_{0}\right)+ & \sum_{i=1}^{d} \partial_{x_{i}} f\left(X_{-}\right) \cdot X_{t}^{i}+\frac{1}{2} \sum_{i, j=1}^{d} \partial_{x_{i} x_{j}}^{2} f\left(X_{-}\right) \cdot\left\langle\left(X^{i}\right)^{c},\left(X^{j}\right)^{c}\right\rangle_{t} \\
& +\sum_{0 \leq s \leq t}\left\{f\left(X_{s}\right)-f\left(X_{s-}\right)-\sum_{i=1}^{d} \partial_{x_{i}} f\left(X_{s-}\right) \Delta X_{s}^{i}\right\},
\end{aligned}
$$

where the symbols $\partial_{x_{i}}$ and $\partial_{x_{i} x_{j}}^{2}$ denote the partial derivative and the second partial derivative, respectively.

An important corollary to Theorem 1.3.7 is the so called formula of integration by parts. It states that for two semimartingales $X$ and $Y$ the identity

$$
X Y=X_{0} Y_{0}+X_{-} \cdot Y+Y_{-} \cdot X+[X, Y]
$$

holds. To prove it, it suffices to apply Theorem 1.3 .7 to the function $f(x, y):=x y$ and compare the result to (1.12).

Itô's formula can be applied for two-times continuously differentiable transformations of a semimartingale. Nevertheless, it is sometimes important to establish if some less regular transformations of a semimartingale preserve the semimartingale property. The next lemma gives an answer to this question in a special case which we shall meet later.

1.3.8 Lemma. Let $X$ be a semimartingale that does not vanish a.s. Then $\frac{1}{X}$ is again a semimartingale.

Proof. We cannot apply directly Itô's formula because the function $f(x):=\frac{1}{x}$ is not two-times continuously differentiable on $\mathbb{R}$. We choose two-time continuously 
differentiable functions $f_{n}, n \geq 1$, such that $f_{n}(x)=f(x)$ on $\left\{|x| \geq \frac{1}{n}\right\}$. By Theorem 1.3.7, $f_{n}(X)$ is a semimartingale, for every $n \geq 1$. Now we define the sequence of stopping times $\left(T_{n}\right)_{n \in \mathbb{N}}$ by $T_{n}:=\inf \left\{t \geq 0:\left|X_{t}\right|<\frac{1}{n}\right\}, n \in \mathbb{N}$. Then $T_{n} \uparrow+\infty$ as $n \rightarrow+\infty$ because $X$ does not vanish. Moreover, $1_{\left[0, T_{n}\right)} f_{n}(X)$ is a semimartingale and so

$$
f(X)^{T_{n}}=\left(\frac{1}{X}\right)^{T_{n}}=1_{\left[0, T_{n}\right)} \frac{1}{X}+\frac{1}{X_{T_{n}}} 1_{\left[T_{n},+\infty\right)}=1_{\left[0, T_{n}\right)} f_{n}(X)+\frac{1}{X_{T_{n}}} 1_{\left[T_{n},+\infty\right)}
$$

is a semimartingale. Therefore we can conclude that $f(X)$ is a semimartingale too (cf. Protter (2005), Theorem II.6 and its corollary).

\subsection{Stable Subspaces of Martingales}

We recall that we fixed a complete probability space $(\Omega, \mathscr{F}, \mathbb{P})$ and a filtration $\mathbb{F}$ satisfying the usual conditions. The aim of this section is to introduce a general definition of predictable representation property (PRP) for an arbitrary family of local martingales $\mathscr{X} \subseteq \mathscr{M}_{\mathrm{loc}, 0}(\mathbb{F})$. To achieve this goal we use the notion of generated stable subspaces. The following definition is as Jacod (1979), Definition 4.1.

1.4.1 Definition. Let $q \in[1,+\infty)$. A linear subspace $\mathscr{H}$ of $\mathscr{H}_{0}^{q}$ is called a stable subspace of $\mathscr{H}_{0}^{q}$ if:

(i) $\mathscr{H}$ is closed in $\left(\mathscr{H}_{0}^{q},\|\cdot\|_{\mathscr{H}^{q}}\right)$;

(ii) $\mathscr{H}$ is stable under stopping, that is, $M^{T} \in \mathscr{H}$ for every stopping time $T$ and every $M \in \mathscr{H}$.

The terminology "stable subspace" comes from the stability under stopping. On the other side, the stopping operation is a particular case of stochastic integration. Indeed, as we observed at p. 9, for any stopping time $T$ the process $1_{[0, T]}$ is predictable and bounded. Hence for any $M \in \mathscr{H}_{0}^{q}$ and for any stopping time $T$ we have $1_{[0, T]} \in \mathrm{L}^{q}(M)$ and from formula (1.31) we get

$$
M^{T}=1_{[0, T]} \cdot M
$$

From the previous formula, with the help of a monotone class argument (we recall that the stochastic intervals of the form $[0, T]$ generate the predictable $\sigma$-algebra $\mathscr{P}$, cf. Jacod \& Shiryaev (2000), Theorem I.2.2), it is immediate to obtain that a stable subspace in $\mathscr{H}_{0}^{q}$ is stable under stochastic integration of locally bounded predictable processes, i.e., $H \cdot M \in \mathscr{H}$ if $M \in \mathscr{H}$ and $H$ is locally bounded and predictable. More generally, the following proposition holds (cf. Jacod (1979), Proposition 4.3).

1.4.2 Proposition. A linear subspace $\mathscr{H}$ of $\mathscr{H}_{0}^{q}$ is a stable subspace of $\mathscr{H}_{0}^{q}$ if and only if it is closed in $\left(\mathscr{H}_{0}^{q},\|\cdot\|_{\mathscr{H}^{q}}\right)$ and stable under stochastic integration, i.e., $H \cdot M \in \mathscr{H}$ for every $M \in \mathscr{H}$ and $H \in \mathrm{L}^{q}(M)$.

The definition of stable subspace in $\mathscr{H}_{0}^{q}$ leads to the notion of generated stable subspace in $\mathscr{H}_{0}^{q}$ by a family $\mathscr{X} \subseteq \mathscr{M}_{\text {loc }, 0}$. 
1.4.3 Definition. Let $\mathscr{X} \subseteq \mathscr{M}_{\mathrm{loc}, 0}$ and $q \in[0,+\infty)$. We denote the stable subspace generated by $\mathscr{X}$ in $\mathscr{H}_{0}^{q}$ by $\mathscr{L}^{q}(\mathscr{X})$ and we define it as it follows:

(i) If $\mathscr{X} \subseteq \mathscr{H}_{0}^{q}, \mathscr{L}^{q}(\mathscr{X})$ is the smallest stable subspace of $\mathscr{H}_{0}^{q}$ containing $\mathscr{X}$.

(ii) If $\mathscr{X} \subseteq \mathscr{M}_{\mathrm{loc}, 0}, \mathscr{L}^{q}(\mathscr{X})$ is defined as the stable subspace generated in $\mathscr{H}_{0}^{q}$ by $\mathscr{X}_{q}:=\left\{H \cdot M: H \in \mathrm{L}^{q}(M), M \in \mathscr{X}\right\}$, i.e., $\mathscr{L}^{q}(\mathscr{X}):=\mathscr{L}^{q}\left(\mathscr{X}_{q}\right)$.

If $\mathscr{X} \subseteq \mathscr{H}_{0}^{q}$, the definition of stable subspace generated by $\mathscr{X}$ in $\mathscr{H}_{0}^{q}$ is wellposed because the arbitrary intersection of stable is again a stable subspace of $\mathscr{H}_{0}^{q}$. We notice that, from Proposition 1.3.4.(ii), the system $\mathscr{X}_{q}$ introduced in Definition 1.4.3, is contained in $\mathscr{H}_{0}^{q}$, for any $\mathscr{X} \subseteq \mathscr{M}_{\text {loc }, 0}$. Hence, for every family $\mathscr{X} \subseteq \mathscr{M}_{\text {loc }, 0}$, $\mathscr{L}^{q}(\mathscr{X})$ is well defined and it is a stable subspace of $\mathscr{H}_{0}^{q}$. Nevertheless, it is not true, in general, that $\mathscr{X} \subseteq \mathscr{L}^{q}(\mathscr{X})$ and, in the extreme case, it can happen that $\mathscr{L}^{q}(\mathscr{X})$ contains only the null martingale (cf. Jacod (1979), Exercise 4.1).

If $\mathscr{X} \subseteq \mathscr{H}_{\mathrm{loc}, 0}^{q}, q \in[1,+\infty)$, we can give a nice characterization of the stable subspace generated by $\mathscr{X}$ in $\mathscr{H}_{0}^{q}$.

1.4.4 Lemma. Let $\mathscr{X} \subseteq \mathscr{H}_{\mathrm{loc}, 0}^{q}$. Then $\mathscr{L}^{q}(\mathscr{X})$ is the smallest stable subspace of $\mathscr{H}_{0}^{q}$ containing $\mathscr{H}:=\left\{M^{T}: M \in \mathscr{X}, T\right.$ stopping time such that $\left.M^{T} \in \mathscr{H}_{0}^{q}\right\}$.

Proof. Clearly $\mathscr{H} \subseteq \mathscr{H}_{0}^{q}$, hence, by Definition $1.4 .3, \mathscr{L}^{q}(\mathscr{H})$ is the smallest stable subspace of $\mathscr{H}_{0}^{q}$ containing $\mathscr{H}$. Because of $\mathscr{X} \subseteq \mathscr{M}_{\mathrm{loc}, 0}, \mathscr{L}^{q}(\mathscr{X})$ is the smallest stable subspace of $\mathscr{H}_{0}^{q}$ containing $\mathscr{X}_{q}$, where $\mathscr{X}_{q}$ is as in Definition 1.4.3. From Proposition 1.4.2, $\mathscr{H} \subseteq \mathscr{X}_{q}$ because for every stopping time $T, 1_{[0, T]} \in \mathrm{L}^{q}(M)$ and $M^{T}=1_{[0, T]} \cdot M$. But this implies $\mathscr{L}^{q}(\mathscr{H}) \subseteq \mathscr{L}^{q}\left(\mathscr{X}_{q}\right)=: \mathscr{L}^{q}(\mathscr{X})$ because $\mathscr{L}^{q}(\mathscr{X})$ is a stable subspace. Now we verify the inclusion $\mathscr{X}_{q} \subseteq \mathscr{L}^{q}(\mathscr{H})$. If $X \in \mathscr{X}_{q}$ there exists $M \in \mathscr{X}$ and $H \in \mathrm{L}^{q}(M)$ such that $X=H \cdot M$. We choose a sequence $\left(T_{n}\right)_{n \in \mathbb{N}}$ of stopping times reducing $M$ to $\mathscr{H}_{0}^{q}$ and put $X^{n}:=X^{T_{n}}$. From Proposition 1.4.2, we have $X^{n} \in \mathscr{L}^{q}(\mathscr{H})$ because $X^{n}=(H \cdot M)^{T_{n}}=H \cdot M^{T_{n}}$ and $M^{T_{n}} \in \mathscr{H}$ for every $n \in \mathbb{N}$. On the other side, $X^{n}=H 1_{\left[0, T_{n}\right]} \cdot M$. If we put $H^{n}:=H 1_{\left[0, T_{n}\right]}, H^{n}$ converges pointwise to $H$ and $\left|H^{n}\right| \leq|H| \in \mathrm{L}^{q}(M)$. Proposition 1.3 .5 implies that $X^{n}$ converges to $X$ in $\left(\mathscr{H}_{0}^{q},\|\cdot\|_{\mathscr{H} q}\right)$. Hence $X \in \mathscr{L}^{q}(\mathscr{H})$ because $\mathscr{L}^{q}(\mathscr{H})$ is closed in $\left(\mathscr{H}_{0}^{q},\|\cdot\|_{\mathscr{H}^{q}}\right)$. In conclusion, $\mathscr{X}_{q} \subseteq \mathscr{L}^{q}(\mathscr{H})$ and by the stability of $\mathscr{L}^{q}(\mathscr{H})$, we get $\mathscr{L}^{q}(\mathscr{X}):=\mathscr{L}^{q}\left(\mathscr{X}_{q}\right) \subseteq \mathscr{L}^{q}(\mathscr{H})$.

We now formulate a result which gives a complete description of $\mathscr{L}^{q}(\mathscr{X})$ for $\mathscr{X} \subseteq \mathscr{M}_{\mathrm{loc}, 0}$. We refer to Jacod (1979), Theorem 4.6 and Proposition 4.7

1.4.5 Theorem. (i) If $M \in \mathscr{M}_{\mathrm{loc}, 0}$, then $\mathscr{L}^{q}(M)=\left\{H \cdot M, H \in \mathrm{L}^{q}(M)\right\}$.

(ii) If $\mathscr{X} \subseteq \mathscr{M}_{\mathrm{loc}, 0}$, then

$$
\mathscr{L}^{q}(\mathscr{X})=\overline{\operatorname{Span}\left(\bigcup_{M \in \mathscr{X}} \mathscr{L}^{q}(M)\right)}{ }^{\left(\mathscr{H}_{0}^{q},\|\cdot\|_{\left.\mathscr{H}^{q}\right)}\right.}
$$

In particular, from (i) it follows that every $X \in \mathscr{L}^{q}(\mathscr{X})$ can be represented as

$$
X=\lim _{n \rightarrow \infty} \sum_{k=1}^{m_{n}} H^{n, k} \cdot M^{n, k}, \quad H^{n, k} \in \mathrm{L}^{q}\left(M^{n, k}\right), M^{n, k} \in \mathscr{X}, \quad k, n \geq 1,
$$

where the limit is taken in $\left(\mathscr{H}_{0}^{q},\|\cdot\|_{\mathscr{H}^{q}}\right)$. 
Let $M, N \in \mathscr{M}_{\text {loc }, 0}$ be orthogonal. If we consider $H \in \mathrm{L}_{\text {loc }}^{1}(M)$ and $K \in \mathrm{L}_{\text {loc }}^{1}(N)$ the stochastic integrals $X:=H \cdot M$ and $Y:=K \cdot M$ need not to be again orthogonal local martingales. The following lemma gives conditions on $H$ and $K$ to preserve the orthogonality of $H \cdot M$ and $K \cdot N$.

1.4.6 Lemma. Let $M, N \in \mathscr{M}_{\mathrm{loc}, 0}$ be orthogonal local martingales.

(i) If $q \in(1,+\infty)$ and $p$ is its conjugate exponent, then $\mathscr{L}^{p}(N) \perp \mathscr{L}^{q}(M)$. Furthermore, for every $H \in \mathrm{L}^{q}(M)$ and $K \in \mathrm{L}^{p}(N)$ the process $H K \cdot[M, N]$ belongs to $\mathscr{M}_{0}$ and the process $|H K| \cdot \operatorname{Var}([M, N])$ is integrable.

(ii) If $N \in B M O_{0}$, then $N \perp \mathscr{L}^{1}(M)$. Furthermore, for every $H \in \mathrm{L}^{1}(M)$ the process $H \cdot[M, N]$ belongs to $\mathscr{M}_{0}$ and the process $|H| \cdot \operatorname{Var}([M, N])$ is integrable.

Proof. We show the first statement. Let $q>1$ and $p>1$ be its conjugate exponent. For $H \in \mathrm{L}^{q}(M)$ and $K \in \mathrm{L}^{p}(N)$, by Proposition 1.3.4.(ii), we have $H \cdot M \in \mathscr{H}_{0}^{q}$ and $K \cdot N \in \mathscr{H}_{0}^{p}$. By Kunita-Watanabe's inequality with $U=1$ (cf. (1.21)) and by Proposition 1.3.4.(i), we get

$$
\begin{aligned}
\mathbb{E}\left[\operatorname{Var}([H \cdot M, K \cdot N])_{\infty}\right] & \leq\left\|\left([H \cdot M, H \cdot M]_{\infty}\right)^{\frac{1}{2}}\right\|_{q}\left\|\left([K \cdot N, K \cdot N]_{\infty}\right)^{\frac{1}{2}}\right\|_{p} \\
& \leq\left\|\left(H^{2} \cdot[M, M]_{\infty}\right)^{\frac{1}{2}}\right\|_{q}\left\|\left(K^{2} \cdot[N, N]_{\infty}\right)^{\frac{1}{2}}\right\|_{p} \\
& =\|H\|_{L^{q}(M)}\|K\|_{L^{q}(N)}<+\infty .
\end{aligned}
$$

Hence $[H \cdot M, K \cdot N] \in \mathscr{A}$. Moreover, the process of finite variation $[M, N]$ belongs to $\mathscr{M}_{\text {loc }, 0}$ because of the orthogonality of $M$ and $N$ (cf. Proposition 1.2.11.(i)). By Proposition 1.3.4.(i), we have $[H \cdot M, K \cdot N]=H K \cdot[M, N]$. In particular, this means that the process of finite variation $H K \cdot[M, N]$ belongs to $\mathscr{A}$. Because $[M, N]$ is a local martingale of finite variation, we can apply Proposition 1.2.5.(iii) to deduce that $H K \cdot[M, N]$ is a local martingale. But then also $[H \cdot M, K \cdot N]$ is a local martingale and this yields $K \cdot N \perp H \cdot M$ (cf. Proposition 1.2.11.(i)). Because of Theorem 1.4.5.(i) we can conclude that $\mathscr{L}^{p}(N) \perp \mathscr{L}^{q}(M)$. We have proven that the process $H K \cdot[M, N]$ belongs to $\mathscr{M}_{\mathrm{loc}, 0} \cap \mathscr{A}$ and so, by Proposition 1.2.5.(ii), it belongs to $\mathscr{M}_{0}$. From $\operatorname{Var}([H \cdot M, K \cdot N])=|H K| \cdot \operatorname{Var}([M, N])$ and (1.36) it follows that $|H K| \cdot \operatorname{Var}([M, N])$ is integrable and the proof is concluded. Part (ii) can be verified in a similar way applying Fefferman's inequality (cf. (1.22)) instead of Kunita-Watanabe's inequality.

In Theorem 1.4.5 we gave a complete description of the stable subspace generated in $\mathscr{H}_{0}^{q}, q \geq 1$, by an arbitrary family $\mathscr{X} \subseteq \mathscr{M}_{\text {loc }, 0}$. We now assume that $\mathscr{X}$ is $a$ countable family of orthogonal local martingales contained in $\mathscr{H}_{\mathrm{loc}, 0}^{2}$ and we show that the structure of the generated stable subspace $\mathscr{L}^{2}(\mathscr{X})$ becomes much simpler than that one expressed in Theorem 1.4.5. We need a preliminary lemma which is purely analytical.

1.4.7 Lemma. Let $\mathscr{H}$ be a Hilbert space with the scalar product $(\cdot, \cdot)_{\mathscr{H}}$ and let $\|\cdot\|_{\mathscr{H}}$ be the norm induced by the scalar product. If $\left(\mathscr{H}_{n}\right)_{n \geq 1} \subseteq \mathscr{H}$ is a sequence of closed pairwise orthogonal linear subspaces of $\mathscr{H}$, then the linear subspace $\tilde{\mathscr{H}}:=\bigoplus_{n=1}^{\infty} \mathscr{H}_{n}$ is closed in $\left(\mathscr{H},\|\cdot\|_{\mathscr{H}}\right)$. 
Proof. Let $\left(x^{i}\right)_{i \geq 1} \subseteq \tilde{\mathscr{H}}$ be such that $x^{i} \longrightarrow x$ in $\left(\mathscr{H},\|\cdot\|_{\mathscr{H}}\right)$ as $i \rightarrow+\infty$. We have to show that $x \in \tilde{\mathscr{H}}$. Because of $x^{i} \in \tilde{\mathscr{H}}$, we can write it in a unique way as $x^{i}=\sum_{n=1}^{\infty} x_{n}^{i}$, where $x_{n}^{i} \in \mathscr{H}_{n}$ and $n \geq 1$. The sequence $\left(x_{n}^{i}\right)_{i \geq 1} \subseteq \mathscr{H}_{n}$ is a Cauchy sequence in $\mathscr{H}$. Indeed, because of the pairwise orthogonality of $\left(\mathscr{H}_{n}\right)_{n \geq 1}$

$$
\left\|x_{n}^{i}-x_{n}^{j}\right\|_{\mathscr{H}}^{2} \leq \sum_{n=1}^{\infty}\left\|x_{n}^{i}-x_{n}^{j}\right\|_{\mathscr{H}}^{2} \leq\left\|x^{i}-x^{j}\right\|_{\mathscr{H}}^{2} \longrightarrow 0 \quad \text { as } i, j \rightarrow+\infty .
$$

The subspace $\mathscr{H}_{n}$ is closed and so there exists $\tilde{x}_{n} \in \mathscr{H}_{n}$ such that $x_{n}^{i} \longrightarrow \tilde{x}_{n}$ in $\mathscr{H}$ as $i \rightarrow+\infty$. Fatou's lemma implies that

$$
\sum_{n=1}^{\infty}\left\|x_{n}^{i}-\tilde{x}_{n}\right\|_{\mathscr{H}}^{2} \leq \liminf _{j \rightarrow+\infty} \sum_{n=1}^{\infty}\left\|x_{n}^{i}-x_{n}^{j}\right\|_{\mathscr{H}}^{2} \longrightarrow 0 \quad \text { as } i \rightarrow+\infty
$$

and, if $i$ is big enough, it follows

$$
\sum_{n=1}^{\infty}\left\|\tilde{x}_{n}\right\|_{\mathscr{H}}^{2} \leq 2\left(\sum_{n=1}^{\infty}\left\|x_{n}^{i}-\tilde{x}_{n}\right\|_{\mathscr{H}}^{2}+\sum_{n=1}^{\infty}\left\|x_{n}^{i}\right\|_{\mathscr{H}}^{2}\right)<+\infty .
$$

Then we can put

$$
\tilde{x}:=\sum_{n=1}^{\infty} \tilde{x}_{n}, \quad \tilde{x}_{n} \in \mathscr{H}_{n}
$$

and, by definition, $\tilde{x} \in \tilde{\mathscr{H}}$. Note that the left-hand side of (1.37) is just $\left\|x^{i}-\tilde{x}\right\|_{\mathscr{H}}$. Because of (1.37), $x^{i} \longrightarrow \tilde{x}$ in $\mathscr{H}$ as $i \rightarrow+\infty$ and by the uniqueness of the limit we conclude $x=\tilde{x} \in \tilde{\mathscr{H}}$.

Now we apply Lemma 1.4.7 to describe the structure of the stable subspace generated in $\mathscr{H}_{0}^{2}$ by $\mathscr{X} \subseteq \mathscr{H}_{\text {loc }, 0}^{2}$. We recall that the terminology "strongly orthogonal" is used to denote orthogonal local martingale in case of ambiguity with the usual orthogonality in a Hilbert space (cf. §1.2.7).

1.4.8 Theorem. Let $\mathscr{X}=\left\{M^{1}, \ldots, M^{n}, \ldots\right\} \subseteq \mathscr{H}_{\mathrm{loc}, 0}^{2}$ be a countable set of strongly orthogonal local martingales. Then $\left(\mathscr{L}^{2}\left(M^{n}\right)\right)_{n \geq 1}$ is a sequence of closed, pairwise strongly orthogonal subspaces of $\left(\mathscr{H}_{0}^{2},\|\cdot\|_{\mathscr{H}^{2}}\right)$ and

$$
\mathscr{L}^{2}(\mathscr{X})=\bigoplus_{n=1}^{\infty} \mathscr{L}^{2}\left(M^{n}\right)
$$

In particular, any $X \in \mathscr{L}^{2}(\mathscr{X})$ is of the form

$$
X=\sum_{n=1}^{\infty} H^{n} \cdot M^{n}, \quad H^{n} \in \mathrm{L}^{2}\left(M^{n}\right), \quad M^{n} \in \mathscr{X}, \quad n \geq 1,
$$

where the limit is taken in $\left(\mathscr{H}_{0}^{2},\|\cdot\|_{\mathscr{H}^{2}}\right)$. 
Proof. We put $\mathscr{H}_{n}:=\mathscr{L}^{2}\left(M^{n}\right)$. Because of Lemma 1.4.6, the sequence $\left(\mathscr{H}_{n}\right)_{n \geq 1} \subseteq$ $\mathscr{H}_{0}^{2}$ is a sequence of closed, pairwise strongly orthogonal subspaces of $\left(\mathscr{H}_{0}^{2},\|\cdot\|_{\mathscr{H}^{2}}\right)$ hence, a sequence of closed, orthogonal subspaces of the Hilbert space $\left(\mathscr{H}_{0}^{2},\|\cdot\|_{2}^{*}\right)$ (the norm $\|\cdot\|_{2}^{*}$ was introduced in (1.4)). We set

$$
\tilde{\mathscr{H}}:=\bigoplus_{n=1}^{\infty} \mathscr{H}_{n}
$$

and, from Lemma 1.4.7, $\tilde{\mathscr{H}}$ is closed in $\left(\mathscr{H}_{0}^{2},\|\cdot\|_{2}^{*}\right)$. Thanks to Doob's inequality (cf. (1.16)) the norms $\|\cdot\|_{2}^{*}$ and $\|\cdot\|_{\mathscr{H}^{2}}$ are equivalent and hence $\tilde{\mathscr{H}}$ is closed also in $\left(\mathscr{H}_{0}^{2},\|\cdot\|_{\mathscr{H}^{2}}\right)$. Obviously, $\tilde{\mathscr{H}}$ is linear and stable under stopping, thus it is a stable subspace of $\left(\mathscr{H}_{0}^{2},\|\cdot\|_{\mathscr{H}^{2}}\right)$. From Theorem 1.4.5.(ii), $\tilde{\mathscr{H}} \subseteq \mathscr{L}^{2}(\mathscr{X})$. We show the converse inclusion. Let $M \in \mathscr{X}$ and let $T$ be a stopping time such that $M^{T} \in \mathscr{H}_{0}^{2}$. Because of (1.31) and Theorem 1.4.5.(i), $M^{T} \in \mathscr{L}^{2}(M)$ and therefore $M^{T} \in \tilde{\mathscr{H}}$. Hence $\tilde{\mathscr{H}}$ is a stable subspace containing

$$
\mathscr{X}^{T}:=\left\{M^{T}: M \in \mathscr{X}, T \text { stopping time such that } M^{T} \in \mathscr{H}_{0}^{2}\right\} .
$$

From Lemma 1.4.4, $\mathscr{L}^{2}(\mathscr{X})$ is the smallest stable subspace of $\left(\mathscr{H}_{0}^{2},\|\cdot\|_{\mathscr{H}^{2}}\right)$ containing $\mathscr{X}^{T}$ so that $\mathscr{L}^{2}(\mathscr{X}) \subseteq \tilde{\mathscr{H}}$ and the identity $\mathscr{L}^{2}(\mathscr{X})=\tilde{\mathscr{H}}$ is proven. Let now $X \in \mathscr{L}^{2}(\mathscr{X})$. By (1.38), $X$ can be written as

$$
X=\sum_{n=1}^{\infty} X^{n}, \quad X^{n} \in \mathscr{H}_{n}, \quad n \geq 1 .
$$

From Theorem 1.4.5.(i), any $X^{n} \in \mathscr{H}_{n}:=\mathscr{L}^{2}\left(M^{n}\right)$ is of the form $X^{n}=H^{n} \cdot M^{n}$ with $H^{n} \in \mathrm{L}^{2}\left(M^{n}\right), n \geq 1$, and (1.39) is proven.

We are ready to give the definition of PRP for a subset $\mathscr{X}$ of $\mathscr{M}_{\mathrm{loc}, 0}$.

1.4.9 Definition. Let $\mathscr{X} \subseteq \mathscr{M}_{\mathrm{loc}, 0}$. We say that $\mathscr{X}$ has the $\mathscr{H}^{q}$-PRP, $q \in[1,+\infty)$, with respect (or relative) to $\mathbb{F}$ if the stable subspace generated by $\mathscr{X}$ in $\mathscr{H}_{0}^{q}$ equals $\mathscr{H}_{0}^{q}$ itself, i.e., $\mathscr{L}^{q}(\mathscr{X})=\mathscr{H}_{0}^{q}$.

We observe that if $\mathscr{X} \subseteq \mathscr{M}_{\text {loc }, 0}$ has the $\mathscr{H}^{q}$-PRP, $q \geq 1$, the decomposition into a direct sum $\mathscr{H}^{q}=\mathscr{L}^{q}(\mathscr{X}) \oplus L^{q}\left(\Omega, \mathscr{F}_{0}, \mathbb{P}\right)$ holds. Indeed, if $M \in \mathscr{H}^{q}$, then $N:=M-M_{0} \in \mathscr{H}_{0}^{q}$ and $M_{0} \in L^{q}\left(\Omega, \mathscr{F}_{0}, \mathbb{P}\right)$. Hence $N \in \mathscr{L}^{q}(\mathscr{X})$ and we can decompose $M$ in a unique way as $M=M_{0}+N$.

Now we investigate the relation between the $\mathscr{H}^{q}$-PRP of a family $\mathscr{X} \subseteq \mathscr{M}_{\text {loc }, 0}$ and the set of local martingales which are orthogonal to $\mathscr{X}$. The next definition was stated in Jacod (1979), Condition 4.9.

1.4.10 Definition. Let $\mathscr{X} \subseteq \mathscr{M}_{\mathrm{loc}, 0}$ and $q \in[1,+\infty]$. We say that $\mathscr{X}$ satisfies condition $C_{q}$ if every martingale $M \in \mathscr{H}_{0}^{q}$ (or, by localization, $M \in \mathscr{H}_{\mathrm{loc}, 0}^{q}$ ), such that $M \perp \mathscr{X}$, is evanescent.

We notice that, if $\mathscr{X} \subseteq \mathscr{M}_{\mathrm{loc}, 0}$ has the $\mathscr{H}^{q}$-PRP, then it has the $\mathscr{H}^{p}$-PRP, for every $p \in[1, q]$. Moreover, if $\mathscr{X}$ satisfies condition $C_{q}$, then it satisfies also condition $C_{p}$, for every $p \in[q,+\infty]$. These results are easy to prove and are established in 
Jacod (1979), Proposition 4.10. The relation between the conditions $C_{q}$ and the $\mathscr{H}^{q}$-predictable representation properties is given by the following theorem. For the proof cf. Jacod (1979), Theorem 4.11.

1.4.11 Theorem. Let $\mathscr{X} \subseteq \mathscr{M}_{\mathrm{loc}, 0}$, and $q \in[1,+\infty)$ with conjugate exponent $p \in(1,+\infty]$.

(i) If $\mathscr{X}$ has the $\mathscr{H}^{q}-P R P$, then it also satisfies condition $C_{p}$.

(ii) $\mathscr{X} \subseteq \mathscr{H}_{\mathrm{loc}, 0}^{q}$ has the $\mathscr{H}^{q}-P R P$ if and only if it satisfies condition $C_{p}$.

We stress that $\mathscr{X} \subseteq \mathscr{M}_{\text {loc }, 0}$ satisfies condition $C_{\infty}$ if and only if it possesses the $\mathscr{H}^{1}$-PRP. Indeed, $\mathscr{H}_{\text {loc }, 0}^{1}$ coincides with $\mathscr{M}_{\text {loc }, 0}$ (cf. Jacod (1979), Lemma 2.38) and the assertion follows from Theorem 1.4.11.(ii).

Condition $C_{1}$ is the strongest of the conditions $C_{q}$ (cf. Jacod (1979), Proposition 4.10), i.e., if $\mathscr{X}$ satisfies condition $C_{1}$, all the conditions $C_{q}$ are equivalent. If $\mathscr{X} \subseteq \mathscr{H}_{\mathrm{loc}, 0}^{q}$ for every $q \geq 1$, e.g. $\mathscr{X} \subseteq \mathscr{H}_{\mathrm{loc}, 0}^{\infty}$, and it satisfies condition $C_{1}$, then from Theorem 1.4.11, $\mathscr{X}$ has the $\mathscr{H}^{q}$-PRP, for every $q \in[1,+\infty)$. Examples of families $\mathscr{X} \subseteq \mathscr{M}_{\mathrm{loc}, 0}$ satisfying condition $C_{1}$ are the case in which $\mathscr{X} \subseteq \mathscr{M}_{\mathrm{loc}, 0}^{c}$ or the one in which $\mathscr{X}$ consists of finitely many local martingales, as showed in Jacod (1979), Proposition 4.13 and 4.67.

The problem of finding sufficient conditions for a family of $\mathscr{M}_{\mathrm{loc}, 0}$ to have the $\mathscr{H}^{1}$-PRP can be approached in an elegant alternative way looking at the solutions of an appropriate martingale problem. Let $(\Omega, \tilde{\mathscr{F}})$ be a measurable space and $\tilde{\mathbb{F}}$ a right-continuous filtration. We consider a family $\mathscr{X}$ of $\tilde{\mathbb{F}}$-adapted processes. We say that a probability measure $\mathbb{P}$ on the measurable space $(\Omega, \tilde{\mathscr{F}})$ is a solution of the martingale problem associated with $\mathscr{X}$ if $\mathscr{X} \subseteq \mathscr{M}_{\text {loc }}(\mathbb{P}, \tilde{\mathbb{F}})$. We denote by $\mathscr{Q}(\mathscr{X})$ the set of all the probability measures on $(\Omega, \tilde{\mathscr{F}})$ which are solutions of the martingale problem associated with $\mathscr{X}$. We conclude this chapter with a result due to Jacod and Yor (cf. Jacod \& Yor (1977)) which states the connection between the solutions of the martingale problem associated with $\mathscr{X}$ and the $\mathscr{H}^{1}$-PRP. For the formulation we refer to Jacod (1979), Theorem 11.2 and 11.3.

1.4.12 Theorem. Let $\tilde{\mathbb{F}}$ be a right-continuous filtration; $\mathscr{X}$ a family of adapted processes starting at 0 and $\mathbb{P} \in \mathscr{Q}(\mathscr{X})$. Then $\mathscr{L}^{1}(\mathscr{X})=\mathscr{H}_{0}^{1}(\mathbb{P}, \tilde{\mathbb{F}})$ if $\mathscr{F}_{0}$ is trivial and if the the following property holds: Any probability measure $\mathbb{Q}$ on $(\Omega, \tilde{\mathscr{F}})$ which is equivalent to $\mathbb{P}$ on $\tilde{\mathscr{F}}$ and belongs to $\mathscr{Q}(\mathscr{X})$ is in fact identical to $\mathbb{P}$ on $(\Omega, \tilde{\mathscr{F}})$. 
CHAPTER 2

\section{Poisson Random Measures and Lévy Processes}

In $\S 2.1$ we recall some basic properties of stochastically continuous processes with independent increments relative to a filtration. As common in the literature, we call these processes additive processes whereas we call additive processes with homogeneous increments Lévy processes. We refer to He, Wang \& Yan (1992) and Sato (1999). §2.2 concerns Poisson random measures relative to a filtration. In a first step, we introduce the general notion of random measures but then we come to the discussion of random measures which are associated with the jumps of a càdlàg process: We state the definition of Poisson random measure relative to a filtration only for such random measures. For this part we follow Jacod \& Shiryaev (2000), Chapter II. Furthermore, we define the stochastic integral of deterministic functions with respect to a Poisson random measure and a compensated Poisson random measure. For this part the reference books are Kallenberg (1997) and Gihman \& Skorohod (1974). We also show how to construct purely non-Gaussian Lévy processes by integration of deterministic functions with respect to a Poisson random measure. In $\S 2.3$ we prove that the jump measure of a Lévy process relative to a filtration $\mathbb{F}$ is a Poisson random measure relative to $\mathbb{F}$. We conclude the chapter with some well-known results on Lévy processes, as Itô-Lévy decomposition, and describing the structure of the natural filtration (cf. Example 1.2.1) of a Lévy process.

\subsection{Additive Processes and Lévy Processes}

We fix a probability space $(\Omega, \mathscr{F}, \mathbb{P})$ and a filtration $\mathbb{F}$. For the moment, $\mathbb{F}$ is a general filtration and we do not assume that the probability space is complete. We recall that an $\mathbb{F}$-adapted stochastic process $X$ is said to have homogeneous one-dimensional increments if $\left(X_{t}-X_{s}\right)$ is distributed as $X_{t-s}$, for every $0 \leq s \leq t$, while it is said to have independent increments if the random vector $\left(X_{t_{0}}, X_{t_{1}}-X_{t_{0}}, \ldots X_{t_{n}}-X_{t_{n-1}}\right)$ is independent, for every $0 \leq t_{0}<t_{1}<\ldots<t_{n}, n \in \mathbb{N}$. We say that an adapted process $X$ has $\mathbb{F}$-independent increments if $\left(X_{t}-X_{s}\right)$ is independent of $\mathscr{F}_{s}$, for every $0 \leq s \leq t$. Notice that a process with independent and homogeneous one- 
dimensional increments has also homogeneous $n$-dimensional increments, for every $n \geq 1$. In this case we simply say that $X$ has homogeneous increments. To verify that an adapted process with $\mathbb{F}$-independent increments is an $\mathbb{F}$-martingale is a simple task:

2.1.1 Lemma. Let $X$ be an adapted process with $\mathbb{F}$-independent increments such that $X_{0}=0$. Then $X$ is a martingale (not necessarily càdlàg) if and only if the random variable $X_{t}$ is integrable and $\mathbb{E}\left[X_{t}\right]=0$, for every $t \geq 0$.

Proof. If $X$ is a process with $\mathbb{F}$-independent increments such that $X_{0}=0$ and a (not necessarily càdlàg) martingale then $\mathbb{E}\left[X_{t}\right]=\mathbb{E}\left[X_{0}\right]=0, t \geq 0$. Conversely, if $X$ is a process with $\mathbb{F}$-independent increments such that $X_{0}=0$ and that $\mathbb{E}\left[X_{t}\right]=0, t \geq 0$, we get

$$
\mathbb{E}\left[X_{t} \mid \mathscr{F}_{s}\right]=\mathbb{E}\left[X_{t}-X_{s} \mid \mathscr{F}_{s}\right]+X_{s}=\mathbb{E}\left[X_{t}-X_{s}\right]+X_{s}=X_{s}
$$

proving that $X$ is an $\mathbb{F}$-martingale.

The $\mathbb{F}$-independence of the increments and the homogeneity of the one-dimensional increments are stable under convergence in probability, as the following lemma shows.

2.1.2 Lemma. Let $X$ be an $\mathbb{F}$-adapted process. If $\left(X^{n}\right)_{n \geq 1}$ is a sequence of processes with $\mathbb{F}$-independent increments (resp., homogeneous one-dimensional increments) such that $X_{t}^{n}$ converges to $X_{t}$ in probability, for every $t \geq 0$, as $n \rightarrow+\infty$, then $X$ has $\mathbb{F}$-independent increments (resp., homogeneous one-dimensional increments).

Proof. We assume that the sequence $\left(X^{n}\right)_{n \geq 1}$ has $\mathbb{F}$-independent increments (resp., homogeneous one-dimensional increments). For every $0 \leq s \leq t$, we have

$$
\mathbb{E}\left[\mathrm{e}^{i u\left(X_{t}^{n}-X_{s}^{n}\right)} \mid \mathscr{F}_{s}\right]=\mathbb{E}\left[\mathrm{e}^{i u\left(X_{t}^{n}-X_{s}^{n}\right)}\right] \quad\left(\text { resp., } \quad \mathbb{E}\left[\mathrm{e}^{i u\left(X_{t}^{n}-X_{s}^{n}\right)}\right]=\mathbb{E}\left[\mathrm{e}^{i u X_{t-s}^{n}}\right]\right), \quad u \in \mathbb{R} .
$$

Letting $n$ converge to $+\infty$ in the previous formula and applying the theorem of Lebesgue on dominated convergence we get

$$
\mathbb{E}\left[\mathrm{e}^{i u\left(X_{t}-X_{s}\right)} \mid \mathscr{F}_{s}\right]=\mathbb{E}\left[\mathrm{e}^{i u\left(X_{t}-X_{s}\right)}\right], \quad\left(\text { resp. }, \quad \mathbb{E}\left[\mathrm{e}^{i u\left(X_{t}-X_{s}\right)}\right]=\mathbb{E}\left[\mathrm{e}^{i u X_{t-s}}\right]\right), \quad u \in \mathbb{R},
$$

which concludes the proof.

In this work we only consider processes with independent increments which are also stochastically continuous (cf. (1.1)). If $\mathbb{F}$ is a filtration satisfying the usual conditions, a stochastically continuous adapted process with $\mathbb{F}$-independent increments has a unique càdlàg modification which is again a stochastically continuous adapted process with $\mathbb{F}$-independent increments (cf., e.g., He, Wang \& Yan (1992), Theorem 2.68). We observe that any process $X$ with one-dimensional homogeneous increments such that $X_{0}=0$ a.s. and that $X_{t} \longrightarrow 0$ in probability as $t \downarrow 0$ is stochastically continuous. Indeed,

$$
\lim _{s \rightarrow t}\left|X_{t}-X_{s}\right|=\lim _{u \rightarrow 0}\left|X_{u}\right|=0,
$$

where the limits are considered in probability and the equalities in distribution. In particular, any càdlàg process with one-dimensional homogeneous increments which starts at zero is stochastically continuous. 
2.1.3 Definition. Let $X$ be an adapted and stochastically continuous process such that $X_{0}=0$.

(i) We say that $X$ is an additive process in law if it has independent increments.

(ii) If $X$ is a càdlàg additive process in law, we simply call it an additive process.

(iii) We say that $X$ is an additive process in law relative to the filtration $\mathbb{F}$ if it has $\mathbb{F}$-independent increments. If $X$ is also càdlàg, we simply call it an additive process relative to $\mathbb{F}$.

The notation $(X, \mathbb{F})$ emphasizes the filtration relative to which $X$ is an additive process (resp., an additive process in law) and sometimes we simply say that $(X, \mathbb{F})$ is an additive process (resp., an additive process in law) to mean that $X$ is an additive process (resp., an additive process in law) relative to the filtration $\mathbb{F}$. Let $X$ be an additive process (resp., an additive process in law). We use the same notation introduced in Example 1.2.1: $\tilde{\mathbb{F}}^{X}$ is the filtration generated by $X ; \tilde{\mathbb{F}}_{+}^{X}$ is the smallest right-continuous filtration containing $\tilde{\mathbb{F}}^{X} ; \mathbb{F}^{X}$ is the $\mathbb{P}$-completion of $\tilde{\mathbb{F}}^{X}$ in $\mathscr{F} \vee \mathscr{N}(\mathbb{P})$, i.e., $\mathbb{F}^{X}=\left(\tilde{\mathscr{F}}_{t}^{X} \vee \mathscr{N}(\mathbb{P})\right)$, where $\mathscr{N}(\mathbb{P})$ denotes the collection of the $\mathbb{P}$-null sets, and $\mathbb{F}_{+}^{X}$ is the natural filtration of $X$.

2.1.4 Proposition. Let $X$ be an adapted stochastically continuous process such that $X_{0}=0$.

(i) If $X$ is an additive process in law, then $\mathbb{F}^{X}$ satisfies the usual conditions.

(ii) If $(X, \mathbb{F})$ is an additive process in law, then $X$ is an additive process in law.

(iii) The following statements are equivalent:

(a) $X$ is an additive process in law.

(b) $\left(X, \tilde{\mathbb{F}}^{X}\right)$ is an additive process in law.

(c) $\left(X, \tilde{\mathbb{F}}_{+}^{X}\right)$ is an additive process in law.

(d) $\left(X, \mathbb{F}^{X}\right)$ is an additive process in law.

(e) $\left(X, \mathbb{F}_{+}^{X}\right)$ is an additive process in law.

Proof. For the proof of (i) we refer to Wang (1981). The other statements are clear.

A relevant subclass of additive processes, which we are going to introduce, are Lévy processes.

2.1.5 Definition. (i) We say that an additive process (resp., an additive process in law) is a Lévy process (resp., a Lévy process in law) if it has also homogeneous increments.

(ii) We say that an additive process (resp., an additive process in law) relative to $\mathbb{F}$ is a Lévy process (resp., a Lévy process in law) relative to $\mathbb{F}$ if it has also homogeneous increments.

Let $L$ be a Lévy process (resp., a Lévy process in law) relative to $\mathbb{F}$. The notation $(L, \mathbb{F})$ emphasizes the filtration with respect to which $L$ is a Lévy process (resp., a Lévy process in law) and sometimes we simply say that $(L, \mathbb{F})$ is a Lévy process (resp., a Lévy process in law) to mean that $L$ is a Lévy process (resp., a Lévy process in law) relative to $\mathbb{F}$.

A Lévy process with bounded jumps has a finite moment of every order, as the following proposition states. 
2.1.6 Proposition. Let $(L, \mathbb{F})$ be a Lévy process with bounded jumps, i.e., such that $\left|\Delta L_{t}\right| \leq c$, for every $t \geq 0$, a.s. Then for every $t \geq 0$, the random variable $L_{t}^{m}$ belongs to $L^{1}(\mathbb{P})$, for every $m \in \mathbb{N}$, thus,

$$
\mathbb{E}\left[\left|L_{t}\right|^{m}\right]<+\infty, \quad t \geq 0, \quad m \in \mathbb{N} .
$$

The proof of Proposition 2.1.6 can be find in Appelbaum (2009), Theorem 2.4.7 and it is given in an elementary way, only making use of the strong Markov property of a Lévy process with respect to its natural filtration.

We now assume that the filtration $\mathbb{F}$ satisfies the usual conditions. We also require that the probability space $(\Omega, \mathscr{F}, \mathbb{P})$ is complete. We keep these assumptions for all the remaining part of this chapter. First examples of additive processes relative to $\mathbb{F}$ are the Poisson process and the Wiener process, while the homogeneous Poisson process and the standard Wiener process are examples of Lévy processes relative to $\mathbb{F}$ (cf. $\S 1.2 .4$ and $\S 1.2 .6)$.

For an additive process in law $X$, we denote by $\varphi$ the characteristic function of $X$, i.e.,

$$
\varphi_{t}(u):=\mathbb{E}\left[\exp \left(i u X_{t}\right)\right], \quad t \geq 0, \quad u \in \mathbb{R} .
$$

Because of the stochastic continuity, it is easy to see that the characteristic function $\varphi$ of an additive process $X$ is continuous in both the variables $t$ and $u$. Additive processes in law are processes with infinitely divisible distribution. We do not go further into details but we only recall that a well-known property of infinitely divisible distribution is that $\varphi_{t}(u) \neq 0, u \in \mathbb{R}, t \geq 0$ (cf. He, Wang \& Yan (1992), Lemma 2.65 or also Sato (1999), Lemma 7.5). Let $X$ be an additive process relative to $\mathbb{F}$. We introduce the process $Z=\left(Z_{t}\right)_{t \geq 0}$ by

$$
Z_{t}:=\varphi_{t}(u)^{-1} \exp \left(i u X_{t}\right), \quad t \geq 0, \quad u \in \mathbb{R} .
$$

Because $\varphi_{t}(u) \neq 0,(2.2)$ is well posed. We remark that the process $Z$ depends also on the parameter $u$ and so we should write $Z(u)$ but we write only $Z$ to keep notations simpler. In He, Wang \& Yan (1992), Lemma 2.66, it is proven that the process $Z$ is an $\mathbb{F}$-martingale. We call the process $Z$ the martingale associated with $X$.

While Lévy processes are always semimartingales, as we shall see later, the same is not true for additive processes. However, necessary and sufficient conditions can be given for an additive process $(X, \mathbb{F})$ to be a semimartingale:

2.1.7 Theorem. Let $(X, \mathbb{F})$ be an additive process. If it is a semimartingale, then for all $u \in \mathbb{R}$ the function $t \mapsto \varphi_{t}(u)$ is of finite variation. Conversely, if for some $u \neq 0, t \mapsto \varphi_{t}(u)$ is of finite variation, then $X$ is a semimartingale.

Proof. This result is formulated in He, Wang \& Yan (1992), Theorem 11.34. However, they only show that if for some $u \neq 0$, the function $t \mapsto \varphi_{t}(u)$ is of finite variation, then $X$ is a semimartingale and we do not verify it again. The other implication of the theorem is left as an exercise and therefore we give now a proof of it. Assume that $X$ is a semimartingale. Because of Itô's formula $\exp (i u X)$ is a semimartingale. On the other side, $Z_{t} \neq 0$ because of its definition and $\varphi_{t}(u)=\frac{\exp \left(i u X_{t}\right)}{Z_{t}}$. 
By Lemma 1.3.8 (adapted to complex-valued processes), $\frac{1}{Z}$ is a semimartingale and Itô's formula implies that $\left(\varphi_{t}(u)\right)_{t \geq 0}$ is a semimartingale. By Jacod \& Shiryaev (2000), Proposition I.4.27, it is of finite variation.

We now formulate a theorem which gives sufficient conditions for a family of additive processes which are also semimartingales to be independent.

2.1.8 Theorem. Let $X^{1}, \ldots, X^{m}$ be semimartingales and additive processes relative to $\mathbb{F}$. If

$$
\left[X^{j}, X^{k}\right]=0, \quad j, k=1, \ldots, m ; j \neq k,
$$

then the random vector $\left(X_{t}^{1}-X_{s}^{1}, \ldots, X_{t}^{m}-X_{s}^{m}\right)$ is independent of $\mathscr{F}_{s}$, for every $0 \leq s \leq t$. Moreover, the vector $\left(X^{1}, \ldots, X^{m}\right)$ of additive processes is independent.

Theorem 2.1.8 is of great importance for the further development. On the other side, a detailed proof of it requires some preparation and several preliminary lemmas. For this reason we postpone the discussion of its proof to Appendix A. A similar result can be found in He, Wang \& Yan (1992), Theorem 11.43 but the statement of Theorem 2.1.8 and the proof which we shall give differs in some parts from He, Wang \& Yan (1992).

\subsection{Poisson Random Measures}

We devote this section to Poisson random measures relative to a filtration. We do not consider general Poisson random measures. Rather we restrict our attention to random measures associated with the jumps of adapted càdlàg processes and consider only the homogeneous case. Before we need to introduce the notion of random measure and of integer-valued random measure. Of particular interest will be the part concerning the definition of the stochastic integral of deterministic functions with respect to a Poisson random measure and with respect to a compensated Poisson random measure. We recall that we fixed a complete probability space $(\Omega, \mathscr{F}, \mathbb{P})$ and a filtration $\mathbb{F}$ satisfying the usual conditions. For the sake of simplicity, we introduce the following notation:

$$
(E, \mathscr{B}(E)):=\left(\mathbb{R}_{+} \times \mathbb{R}, \mathscr{B}\left(\mathbb{R}_{+}\right) \otimes \mathscr{B}(\mathbb{R})\right) .
$$

\subsubsection{The Jump Measure of a càdlàg Process}

A random measure $\mathrm{M}$ on $(E, \mathscr{B}(E))$ is a mapping on $\Omega \times \mathscr{B}(E)$ in $[0,+\infty]$ such that:

(i) $\mathrm{M}(\cdot, A)$ is a random variable for every $A \in \mathscr{B}(E)$.

(ii) $\mathrm{M}(\omega, \cdot)$ is a measure on $(E, \mathscr{B}(E))$ such that $\mathrm{M}(\omega ;\{0\} \times \mathbb{R})=0, \omega \in \Omega$.

If $\mathrm{M}$ is a random measure on $(E, \mathscr{B}(E))$, we write

$$
\mathrm{M}(A):=\mathrm{M}(\omega, A), \quad A \in \mathscr{B}(E) .
$$

For any measurable set $A, \mathrm{M}(A)$ is a nonnegative random variable on $(\Omega, \mathscr{F}, \mathbb{P})$. We can therefore introduce the expectation of $\mathrm{M}(A)$ (note that, by definition, $\mathrm{M}(A) \geq$ $0)$. We call intensity measure of $\mathrm{M}$ the mapping $\mathrm{m}$ on $\mathscr{B}(E)$ in $[0,+\infty]$ defined by

$$
\mathrm{m}(A):=\mathbb{E}[\mathrm{M}(A)]
$$


The intensity measure $\mathrm{m}$ is a (deterministic) measure on $(E, \mathscr{B}(E))$. Indeed, $\mathrm{m}(\emptyset)=$ 0 because $\mathrm{M}(\omega, \emptyset)=0$, for every $\omega, \mathrm{M}(\omega, \cdot)$ being a measure. The $\sigma$-additivity of $\mathrm{m}$ follows from the theorem of B. Levi on monotone convergence (cf. Theorem 1.1.4).

We say that a random measure $\mathrm{M}$ on $(E, \mathscr{B}(E))$ is an integer-valued random measure if $\mathrm{M}(A)$ takes values in $\mathbb{N} \cup\{+\infty\}$, for every $A \in \mathscr{B}(E)$. Integer-valued random measures are of special importance because of the relation that they have with càdlàg adapted processes. Let $X$ be a càdlàg adapted process. For every $A \in \mathscr{B}(E)$ we define on $(E, \mathscr{B}(E))$ the random measure M by

$$
\mathrm{M}(\omega ; A)=\sum_{s \geq 0} 1_{\left\{\Delta X_{s}(\omega) \neq 0\right\}} 1_{A}\left(s, \Delta X_{s}(\omega)\right), \quad \omega \in \Omega, \quad A \in \mathscr{B}(E) .
$$

2.2.1 Proposition. Let $X$ be an adapted càdlàg process with values in $\mathbb{R}$. Then the random measure $\mathrm{M}$ defined on $(E, \mathscr{B}(E))$ by (2.5) is an integer-valued random measure.

Proof. Cf. Jacod \& Shiryaev (2000), Proposition I.1.16.

We call the integer-valued random measure $\mathrm{M}$ defined in (2.5) the jump measure of $X$. Let $X$ be an $\mathbb{F}$-adapted càdlàg process and let $\mathrm{M}$ be its jump measure. It is easy to see that $\mathrm{M}(\{t\} \times \mathbb{R}) \in\{0,1\}$. Indeed, from the definition of $\mathrm{M}$, we get

$$
\begin{aligned}
\mathrm{M}(\{t\} \times \mathbb{R}) & =\sum_{s \geq 0} 1_{\left\{\Delta X_{s} \neq 0\right\}} 1_{\{t\} \times \mathbb{R}}\left(s, \Delta X_{s}\right) \\
& =1_{\left\{\Delta X_{t} \neq 0\right\}} 1_{\{t\} \times \mathbb{R}}\left(t, \Delta X_{t}\right) \\
& =1_{\left\{\Delta X_{t} \neq 0\right\}} \in\{0,1\} .
\end{aligned}
$$

If $A \in \mathscr{B}(E)$, we define the process $N^{A}=\left(N_{t}^{A}\right)_{t \geq 0}$ by

$$
N_{t}^{A}:=\mathrm{M}(A \cap[0, t] \times \mathbb{R}) .
$$

2.2.2 Lemma. Let $X$ be an $\mathbb{F}$-adapted càdlàg process and let $\mathrm{M}$ be its jump measure with intensity measure $\mathrm{m}$. If $A \in \mathscr{B}(E)$ is such that $\mathrm{M}(A \cap[0, t] \times \mathbb{R})<+\infty$ for every $t \geq 0$, then the process $N^{A}$ introduced in (2.6) is a simple point process.

Proof. By definition, the process $N^{A}$ is increasing. Proposition 2.2.1 implies that M is an integer-valued random measure and so $N_{t}^{A} \in \mathbb{N}, t \geq 0$. Furthermore, we have $\mathrm{M}(A \cap[0, t] \times \mathbb{R})<+\infty$ for every $t \geq 0$ which yields $\mathrm{M}\left(A \cap\left[0, t+\frac{1}{n}\right] \times \mathbb{R}\right)<+\infty$ for every $n \geq 1$ and $\mathrm{M}\left(A \cap\left[0, t+\frac{1}{n}\right] \times \mathbb{R}\right) \downarrow \mathrm{M}(A \cap[0, t] \times \mathbb{R})$ as $n \rightarrow+\infty$. Therefore $N^{A}$ is right-continuous. The left-limit of $N_{t}^{A}$ is given by $\mathrm{M}(A \cap[0, t) \times \mathbb{R})<+\infty$. Thus, $N^{A}$ is a càdlàg increasing process. For every $t \geq 0, N_{t-}^{A}=\operatorname{M}(A \cap[0, t) \times \mathbb{R})$ and from this it follows that

$$
\Delta N_{t}^{A}=\mathrm{M}(A \cap\{t\} \times \mathbb{R}) \leq \mathrm{M}(\{t\} \times \mathbb{R}) \in\{0,1\} .
$$

We know that $\mathrm{M}(A \cap\{t\} \times \mathbb{R}) \in \mathbb{N}$, because $\mathrm{M}$ is integer-valued and so the previous formula yields $\Delta N^{A} \in\{0,1\}$. It remains to prove that $N^{A}$ is an $\mathbb{F}$-adapted process. To this aim, we recall that for any càdlàg process $X$ the random set $\{\Delta X \neq 0\}$ is a thin set, i.e., there exists a sequence of $\mathbb{F}$-stopping times $\left(T_{n}\right)_{n \geq 1}$ such that

$$
\{\Delta X \neq 0\}=\bigcup_{n=1}^{\infty}\left[T_{n}\right]
$$


and the sequence $\left(T_{n}\right)_{n \geq 1}$ can be chosen is such a way that $\left[T_{n}\right] \cap\left[T_{m}\right]=\emptyset, m \neq n$. The sequence $\left(T_{n}\right)_{n \geq 1}$ is called an exhausting sequence for the jumps of $X$. For this result, cf. Jacod \& Shiryaev (2000), Proposition I.1.32. For $A \in E$ such that $\mathrm{M}(A \cap[0, t] \times \mathbb{R})<+\infty$, we have

$$
\begin{aligned}
N_{t}^{A}=\mathrm{M}(A \cap[0, t] \times \mathbb{R}) & =\sum_{0 \leq s \leq t} 1_{\left\{\Delta X_{s} \neq 0\right\}} 1_{A}\left(s, \Delta X_{s}\right) \\
& =\sum_{n: T_{n} \leq t}^{\infty} 1_{A}\left(T_{n}, \Delta X_{T_{n}}\right),
\end{aligned}
$$

where $\left(T_{n}\right)_{n \geq 1}$ is an exhausting sequence for the jumps of $X$ such that $\left[T_{n}\right] \cap\left[T_{m}\right]=\emptyset$, $m \neq n$. The process $\Delta X$ is an optional process and therefore the random variable $\Delta X_{T_{n}}$ is $\mathscr{F}_{T_{n}}$-measurable, for every $n \geq 1$ (cf. Jacod \& Shiryaev (2000), Proposition I.1.21). Hence $1_{A}\left(T_{n}, \Delta X_{T_{n}}\right)$ is $\mathscr{F}_{T_{n}}$-measurable and therefore $1_{A}\left(T_{n}, \Delta X_{T_{n}}\right) 1_{\left\{T_{n} \leq t\right\}}$ is $\mathscr{F}_{t}$-measurable, because $T_{n}$ is a stopping time for every $n \geq 1$. The proof is now concluded

We notice that if $A \in \mathscr{B}(E)$ is such that $\mathrm{M}(A)<+\infty$, then it satisfies the assumptions of Lemma 2.2.2. However it is not true that if $A \in \mathscr{B}(E)$ is such that $\mathrm{M}(A \cap[0, t] \times \mathbb{R})$ for every $t \geq 0$, then $\mathrm{M}(A)<+\infty$. For example, if we fix $\varepsilon>0$, then the set $\mathbb{R}_{+} \times\{|x|>\varepsilon\}$ has, in general, not finite measure with respect to $\mathrm{M}$. On the other side, $\mathrm{M}([0, t] \times\{|x|>\varepsilon\})<+\infty$ because $\mathrm{M}$ is the jump measure of a càdlàg process. The assumption of Lemma 2.2.2 can be weakened requiring that $\mathrm{M}(A \cap[0, t] \times \mathbb{R})<+\infty$ a.s. for every $t \geq 0$. In this case the process $N^{A}$ introduced in (2.6) is defined only a.s. and therefore the statement of Lemma 2.2.2 holds a.s. To extend the definition of $N^{A}$ everywhere we can set $N_{t}^{A}(\omega)=0$ on the exceptional set on which it is not defined by (2.6). The filtration $\mathbb{F}$ satisfies the usual conditions and this version of the process $N^{A}$ is adapted and càdlàg. We denote this process again by $N^{A}$ and obviously the statement of Lemma 2.2.2 holds also for such a modification.

\subsubsection{Definition of Poisson Random Measures}

We consider an $\mathbb{F}$-adapted càdlàg process $X$ with jump measure $\mathrm{M}$. Let $\mathrm{m}$ be the intensity measure of M. Thanks to Proposition 2.2.1, we know that the random measure $\mathrm{M}$ is an integer-valued random measure. Now we are going to discuss the case in which the jump measure of $X$ is a homogeneous Poisson random measure relative to the filtration $\mathbb{F}$. The definition of a Poisson random measure relative to a filtration can be given in full generality, without relating it to the jump measure of an adapted càdlàg process. Such a general definition requires some technical preparation which would exceed the purpose of this thesis. For the complete treatment of the topic we refer to Jacod \& Shiryaev (2000), Chapter II.

2.2.3 Definition. Let $X$ be an $\mathbb{F}$-adapted process and let $M$ be its jump measure with intensity measure $\mathrm{m}$ (cf. (2.5) and (2.4), respectively). We say that $\mathrm{M}$ is a Poisson random measure relative to the filtration $\mathbb{F}$ if:

(i) The intensity measure $\mathrm{m}$ is of the form $\mathrm{m}=\lambda_{+} \otimes \mu$, where $\lambda_{+}$is the Lebesgue measure on $\left(\mathbb{R}_{+}, \mathscr{B}\left(\mathbb{R}_{+}\right)\right)$and $\mu$ is a $\sigma$-finite measure on $(\mathbb{R}, \mathscr{B}(\mathbb{R}))$.

(ii) For every fixed $s \in \mathbb{R}_{+}$and every $A \in \mathscr{B}(E)$ such that $A \subseteq(s,+\infty) \times \mathbb{R}$, $\mathrm{m}(A)<+\infty$, the random variable $\mathrm{M}(A)$ is independent of $\mathscr{F}_{s}$. 
We remark that if $X$ is a càdlàg process and its jump measure $\mathrm{M}$ is a Poisson random measure relative to $\mathbb{F}$, then the process $X$ has no fixed-time discontinuities a.s. Indeed, we have $\mathrm{m}(\{t\} \times \mathbb{R})=\lambda_{+}(\{t\}) \mu(\mathbb{R})$. Because $\mu$ is $\sigma$-finite, this implies $\mathrm{m}(\{t\} \times \mathbb{R})=0$. Therefore $\mathbb{E}[\mathrm{M}(\{t\} \times \mathbb{R})]=0$ and so $\mathrm{M}(\{t\} \times \mathbb{R})=0$ a.s., $t \geq 0$. The definition of Poisson random measure relative to a filtration may appear different from the usual definition of Poisson random measure (cf., e.g., Sato (1999), Definition 19.1). We are going to prove that a Poisson random measure relative to a filtration is also a Poisson random measure in the usual sense. To this aim the first step that we do is to show that if $\mathrm{M}$ is a Poisson random measure relative to $\mathbb{F}$ and $A \in \mathscr{B}(E)$ is such that $\mathrm{m}(A)<+\infty$, then the process $N^{A}$ introduced in (2.6) is a Poisson process relative to $\mathbb{F}$ (cf. Definition 1.2.6).

2.2.4 Lemma. Let $\mathrm{M}$ be the jump measure of a càdlàg adapted process $X$ with intensity measure $\mathrm{m}$. If $\mathrm{M}$ is a Poisson random measure relative to $\mathbb{F}$, then for every set $A \in \mathscr{B}(E)$ such that $\mathrm{m}(A \cap[0, t] \times \mathbb{R})<+\infty, t \geq 0$, the process $N^{A}$ introduced in $(2.6)$ is an $\mathbb{F}$-adapted Poisson process relative to the filtration $\mathbb{F}$ with intensity function $a^{A}(\cdot):=\mathrm{m}(A \cap[0, \cdot] \times \mathbb{R})$.

Proof. We are going to prove that under the stated assumptions the process $N^{A}$ fulfills all the properties of Definition 1.2.6. Because of $\mathrm{m}(A \cap[0, t] \times \mathbb{R})<+\infty$ for every $t \geq 0$, it follows that $\mathrm{M}(A \cap[0, t] \times \mathbb{R})<+\infty$ a.s. for every $t \geq 0$. From Lemma 2.2.2 and the comment following it, the process $N^{A}$ is an $\mathbb{F}$-adapted simple point process. Moreover, $\mathrm{m}(A \cap[0, t] \times \mathbb{R})<+\infty, t \geq 0$, yields $N_{t}^{A} \in L^{1}(\mathbb{P}), t \geq 0$. Thus,

$$
a^{A}(t):=\mathbb{E}\left[N_{t}^{A}\right]=\mathrm{m}\left(A \cap[0, t] \times \mathbb{R}_{+}\right)=\left(\lambda_{+} \otimes \mu\right)\left(A \cap[0, t] \times \mathbb{R}_{+}\right)<+\infty, \quad t \geq 0 .
$$

The previous equalities imply that $a^{A}(\cdot)$ is a continuous function because $\lambda_{+}$is the Lebesgue measure on $\mathbb{R}_{+}$. It remains to prove that $N^{A}$ has $\mathbb{F}$-independent increments. But this is immediate from the properties of M. Indeed,

$$
N_{t}^{A}-N_{s}^{A}=\mathrm{M}(A \cap(s, t] \times \mathbb{R}), \quad 0 \leq s \leq t,
$$

and $A \cap(s, t] \times \mathbb{R} \subseteq(s,+\infty) \times \mathbb{R}$. Because $\mathrm{M}$ is a Poisson random measure relative to the filtration $\mathbb{F}$, it follows that $N_{t}^{A}-N_{s}^{A}$ is independent of $\mathscr{F}_{s}, 0 \leq s \leq t$. Therefore $\left(N^{A}, \mathbb{F}\right)$ is a Poisson process relative to $\mathbb{F}$ with intensity function $a^{A}(\cdot)$.

We are ready to prove that a Poisson random measure relative to the filtration $\mathbb{F}$ is also a Poisson random measure in the usual sense.

2.2.5 Theorem. Let $\mathrm{M}$ be the jump measure of an $\mathbb{F}$-adapted càdlàg process $X$ with intensity measure $\mathrm{m}$. If $\mathrm{M}$ is a Poisson random measure relative to the filtration $\mathbb{F}$, then it has the following properties:

(i) For every $A \in \mathscr{B}(E)$ such that $\mathrm{m}(A)<+\infty$, the random variable $\mathrm{M}(A)$ is Poisson distributed with parameter $\mathrm{m}(A)$.

(ii) If $A_{1}, \ldots, A_{m}, m \geq 1$, are $\mathscr{B}(E)$-measurable pairwise disjoint subsets such that $\mathrm{m}\left(A_{j}\right)<+\infty, j=1, \ldots, m$, then the vector $\left(\mathrm{M}\left(A_{1}\right), \cdots, \mathrm{M}\left(A_{m}\right)\right)$ of random variable is independent.

(iii) If $A_{1}, \ldots, A_{m}, m \geq 1$, are $\mathscr{B}(E)$-measurable subsets such that $\mathrm{m}\left(A_{j}\right)<+\infty$ and that $A_{j} \subseteq(s,+\infty) \times \mathbb{R}, s>0, j=1, \ldots, m$, then $\left(\mathrm{M}\left(A_{1}\right), \cdots, \mathrm{M}\left(A_{m}\right)\right)$ is a random vector independent of $\mathscr{F}_{s}$. 
Proof. Let $A \in \mathscr{B}(E)$ be such that $\mathrm{m}(A)<+\infty$. We show that $\mathrm{M}(A)$ is Poisson distributed with parameter $\mathrm{m}(A)$. By Lemma 2.2.4, the process $\left(N^{A}, \mathbb{F}\right)$ is a Poisson process with intensity function $a(\cdot)=\mathrm{m}(A \cap[0, \cdot] \times \mathbb{R})$ and therefore, by (1.3)

$$
\mathbb{E}\left[\mathrm{e}^{i u N_{t}^{A}}\right]=\exp \left(\left(\mathrm{e}^{i u}-1\right) \mathrm{m}(A \cap[0, t] \times \mathbb{R})\right) .
$$

When $t \rightarrow+\infty$, applying the theorem of Lebesgue on dominated convergence, we obtain

$$
\mathbb{E}\left[\mathrm{e}^{i u \mathrm{M}(A)}\right]=\exp \left(\left(\mathrm{e}^{i u}-1\right) \mathrm{m}(A)\right), \quad u \in \mathbb{R} .
$$

In particular, this implies that $M(A)$ is Poisson distributed with parameter $\mathrm{m}(A)$ and (i) is proven. Let $A_{1}, \ldots, A_{m} \in \mathscr{B}(E)$ be pairwise disjoint and such that $\mathrm{m}\left(A_{j}\right)<+\infty$. By Lemma 2.2.4, we know that the process $\left(N^{A_{j}}, \mathbb{F}\right)$ is a Poisson process, hence an additive process and a semimartingale (it is a simple point process), $j=1, \ldots, m$. Furthermore,

$$
\begin{aligned}
{\left[N^{A_{j}}, N^{A_{k}}\right]_{t} } & =\sum_{0<s \leq t} \Delta N_{s}^{A_{j}} \Delta N_{s}^{A_{k}} \\
& =\sum_{0<s \leq t} \Delta \mathrm{M}\left(A_{j} \cap[0, s] \times \mathbb{R}\right) \Delta \mathrm{M}\left(A_{k} \cap[0, s] \times \mathbb{R}\right) \\
& =\sum_{0<s \leq t} 1_{\left\{\Delta X_{s} \neq 0\right\}} 1_{A_{j} \cap A_{k}}\left(s, \Delta X_{s}\right)=0, j, k=1, \ldots, m, j \neq k .
\end{aligned}
$$

Now we discuss (ii). If $A_{1}, \ldots, A_{m}$ are pairwise disjoint subsets of $E$ such that $\mathrm{m}\left(A_{j}\right)<+\infty$, because of $(2.8)$ and Theorem 2.1 .8 , the vector $\left(N^{A_{1}}, \ldots N^{A_{m}}\right)$ is an independent vector of Poisson processes and therefore $\left(\mathrm{M}\left(A_{1}\right), \ldots, \mathrm{M}\left(A_{m}\right)\right)=$ $\left(N_{\infty}^{A_{1}}, \ldots, N_{\infty}^{A_{m}}\right)$ is an independent vector of random variables. To see (iii) it is sufficient to prove it for pairwise disjoint sets. Indeed, we can always reduce the general situation to this particular case by considering an appropriate partition of the union of the $A_{j} \mathrm{~s}$. If $A_{1}, \ldots, A_{m}$ are pairwise disjoint subsets of $E$ such that $\mathrm{m}\left(A_{j}\right)<+\infty$ and that $A_{j} \subseteq(s,+\infty) \times \mathbb{R}, j=1, \ldots, m$, because of (2.8) and of Theorem 2.1.8, the vector $\left(N_{t}^{A_{1}}-N_{s}^{A_{1}}, \ldots, N_{t}^{A_{m}}-N_{s}^{A_{m}}\right)$ is independent of $\mathscr{F}_{s}$, $0 \leq s \leq t$. On the other side, $A_{j} \subseteq(s,+\infty) \times \mathbb{R}$ implies $N_{s}^{A_{j}}=0, j=1, \ldots, m$. This yields the vector $\left(N_{t}^{A_{1}}, \ldots, N_{t}^{A_{m}}\right)$ is independent of $\mathscr{F}_{s}, t \geq 0$. Therefore $\left(\mathrm{M}\left(A_{1}\right), \ldots, \mathrm{M}\left(A_{m}\right)\right)=\left(N_{\infty}^{A_{1}}, \ldots, N_{\infty}^{A_{m}}\right)$ is independent of $\mathscr{F}_{s}$.

\subsubsection{The Stochastic Integral for Poisson Random Measures}

Let $X$ be an $\mathbb{F}$-adapted càdlàg process. We assume that that jump measure of $X$ is a Poisson random measure relative to the filtration $\mathbb{F}$ with intensity measure $\mathrm{m}=$ $\lambda_{+} \otimes \mu$. In this section we define the integral of deterministic measurable functions with respect to $\mathrm{M}$. We observe that this part remains valid also if $\mathrm{M}$ is a general Poisson random measure relative to the filtration $\mathbb{F}$ (cf. Jacod \& Shiryaev (2000), Chapter II) and not only a homogeneous Poisson random measure which moreover is the jump measure of an adapted càdlàg process. To simplify the terminology, in this section we call a Poisson random measure relative to a filtration simply a Poisson random measure. We recall that the definition of $(E, \mathscr{B}(E))$ was given in (2.3). For a deterministic numerical function $f$ which is $\mathscr{B}(E)$-measurable we have introduced the notation

$$
\mathrm{m}(f):=\int_{E} f(t, x) \mathrm{m}(\mathrm{d} t, \mathrm{~d} x)
$$


(cf. p.3) if the integral on the right-hand side exists. In particular $\mathrm{m}(f)$ is well defined if $f$ is nonnegative. We define the integral of $f$ with respect to $\mathrm{M} \omega$-wise in an analogous way, because $\mathrm{M}(\omega, \cdot)$ is a (nonnegative) measure on $(E, \mathscr{B}(E))$ for every $\omega \in \Omega$. If $f$ is a nonnegative measurable function, then the integral $\int_{E} f(t, x) \mathrm{M}(\omega, \mathrm{d} t, \mathrm{~d} x)$ always exists. We shall use the notation $\mathrm{M}(f)$ for this random variable with values in $[0,+\infty]$. This definition extends to functions $f$ of arbitrary sign. More precisely, for any measurable function $f$ on $(E, \mathscr{B}(E))$, by $\Omega_{f}$ we denote the set of all $\omega \in \Omega$ such that $\int_{E} f(t, x) \mathrm{M}(\omega, \mathrm{d} t, \mathrm{~d} x)$ exists and is finite a.s. Obviously $\Omega_{f} \in \mathscr{F}$. We say that the integral of $f$ with respect to $M$ exists and is finite a.s. if $\mathbb{P}\left[\Omega_{f}\right]=1$. In this case the random variable $\mathrm{M}(f)$ defined by

$$
\mathrm{M}(\omega, f):=\mathrm{M}(f)(\omega):=\left\{\begin{array}{cc}
\int_{E} f(t, x) \mathrm{M}(\omega, \mathrm{d} t, \mathrm{~d} x), & \text { if } \omega \in \Omega_{f} ; \\
0, & \text { otherwise; }
\end{array}\right.
$$

is called the stochastic integral of $f$ with respect to the Poisson random measure M. Note that the stochastic integral $\mathrm{M}(f)$ exists and is finite a.s. if and only if $\mathrm{M}(|f|)<+\infty$ a.s. We now state the so-called exponential formula (cf. Kallenberg (1997), Lemma 10.2).

2.2.6 Lemma (Exponential Formula). Let $f$ be a function on $(E, \mathscr{B}(E))$. If $f \geq 0$, then

$$
\mathbb{E}\left[\mathrm{e}^{-\mathrm{M}(f)}\right]=\exp \left(\mathrm{m}\left(\mathrm{e}^{-f}-1\right)\right) .
$$

Proof. First we take $f \geq 0$ of the form $f=u 1_{A}$, where $u \geq 0$ and $A \in(E, \mathscr{B}(E))$ is such that $\mathrm{m}(A)<+\infty$. From Theorem 2.2.5, we know that $\mathrm{M}(A)$ is Poisson distributed with parameter $\mathrm{m}(A)$. This implies

$$
\mathbb{E}\left[\mathrm{e}^{-u \mathrm{M}(A)}\right]=\exp \left(\left(\mathrm{e}^{-u}-1\right) \mathrm{m}(A)\right) .
$$

Let now $f$ be a simple function of the form $f=\sum_{j=1}^{m} c_{j} 1_{A_{j}}$ where $c_{j} \geq 0$ for every $j=1, \ldots, m$ and $A_{1}, \ldots, A_{m} \in(E, \mathscr{B}(E))$ are pairwise disjoint sets such that $\mathrm{m}\left(A_{j}\right)<+\infty$, for every $j=1, \ldots, m$. Thanks to Theorem 2.2.5, the random vector $\left(\mathrm{M}\left(A_{1}\right), \ldots, \mathrm{M}\left(A_{m}\right)\right)$ is independent and so, from the previous step, we get

$$
\begin{aligned}
\mathbb{E}\left[\mathrm{e}^{M(-f)}\right] & =\mathbb{E}\left[\exp \left(-\sum_{j=1}^{m} c_{j} \mathrm{M}\left(A_{j}\right)\right)\right] \\
& =\prod_{j=1}^{m} \exp \left(\left(\mathrm{e}^{-c_{j}}-1\right) \mathrm{m}\left(A_{j}\right)\right) \\
& =\exp \left(-\sum_{j=1}^{m}\left(\mathrm{e}^{-c_{j}}-1\right) \mathrm{m}\left(A_{j}\right)\right)=\exp \left(\mathrm{m}\left(\mathrm{e}^{-f}-1\right)\right) .
\end{aligned}
$$

If $f$ is an arbitrary nonnegative function, it can be approximated by an increasing sequence $\left(f_{n}\right)_{n \in \mathbb{N}}$ of simple functions and from the previous step formula (2.10) is satisfied for every $n \in \mathbb{N}$. We conclude the proof passing to the limit and applying the theorem of B. Levi on monotone convergence (cf. Theorem 1.1.4).

Now we characterize, in terms of the intensity measure m, under which conditions the integral of a deterministic function $f$ with respect to $M$ exists and is a.s. finite (cf. Kallenberg (1997), Lemma 10.2). 
2.2.7 Proposition. Let $\mathrm{M}$ be a Poisson random measure on $(E, \mathscr{B}(E))$ with intensity measure $\mathrm{m}$. Then $\mathrm{M}(f)$ exists and is finite a.s. if and only if $\mathrm{m}(|f| \wedge 1)<+\infty$.

Proof. If $\mathrm{M}(f)$ exists and is finite a.s., then, by definition, $\mathrm{M}(|f|)<+\infty$ a.s. Applying Lemma 2.2.6 to $|f|$ and using the estimate $c(|f| \wedge 1) \geq 1-\mathrm{e}^{-f}$ for some constant $c>0$, we deduce $\mathrm{m}(|f| \wedge 1)<+\infty$. Conversely, we now assume that $\mathrm{m}(|f| \wedge 1)<+\infty$. We apply Lemma 2.2 .6 to $c|f|$, where $c>0$ :

$$
\mathbb{E}\left[\mathrm{e}^{-c \mathrm{M}(|f|)}\right]=\exp \left(\mathrm{m}\left(\mathrm{e}^{-c|f|}-1\right)\right) .
$$

Letting $c$ converge to zero on the left-hand side of (2.11) and using the theorem of Lebesgue on dominated convergence, we get $\mathbb{E}\left[\mathrm{e}^{-c \mathrm{M}(|f|)}\right] \longrightarrow \mathbb{P}[\mathrm{M}(|f|)<+\infty]$. For the right-hand side of (2.11) we observe that the inequality

$$
\left|\mathrm{e}^{-c|f|}-1\right| \leq c|f| \wedge 2
$$

holds. Because of $c \downarrow 0$, we can assume $c \in(0,2]$ and so we get $\left|\mathrm{e}^{-c|f|}-1\right| \leq$ $2(|f| \wedge 1)$. We apply again the theorem of Lebesgue on dominated convergence to get $\mathrm{m}\left(\left|\mathrm{e}^{-c|f|}-1\right|\right) \longrightarrow 0$. In conclusion we have

$$
\mathbb{P}[\mathrm{M}(|f|)<+\infty]=1 .
$$

The following lemma can be shown in a similar way.

2.2.8 Lemma. Let $f$ be a function on $(E, \mathscr{B}(E))$. If $\mathrm{m}(|f| \wedge 1)<+\infty$, then

$$
\mathbb{E}\left[\mathrm{e}^{-i \mathrm{M}(f)}\right]=\exp \left(\mathrm{m}\left(\mathrm{e}^{-i f}-1\right)\right) .
$$

We now show how to compute the expectation of the random variable $\mathrm{M}(f)$, where $f$ is a function which belongs to $L^{1}(\mathrm{~m})$.

2.2.9 Lemma. Let $f \in L^{1}(\mathrm{~m})$. Then

$$
\mathbb{E}[\mathrm{M}(f)]=\mathrm{m}(f) .
$$

Moreover, the stochastic integral with respect to $\mathrm{M}$ is a continuous operator on $L^{1}(\mathrm{~m})$ into $L^{1}(\mathbb{P})$.

Proof. For every nonnegative function $f \in L^{1}(\mathrm{~m})$ formula (2.13) holds. Indeed, this is true for indicator functions of the form $1_{A}, A \in \mathscr{B}(E), \mathrm{m}(A)<+\infty$, and hence for nonnegative simple functions $f$. For an arbitrary nonnegative function $f$ we can find a sequence $\left(f_{n}\right)_{n \geq 1}$ of nonnegative simple functions such that $f_{n} \uparrow f$ pointwise as $n \rightarrow+\infty$. The result follows applying the theorem of B. Levi on monotone convergence (cf. Theorem 1.1.4). Clearly, formula (2.13) extends to functions $f$ such that $\mathrm{m}(|f|)<+\infty$. The statement on the continuity follows from

$$
\mathbb{E}[|\mathrm{M}(f)|] \leq \mathbb{E}[\mathrm{M}(|f|)]=\mathrm{m}(|f|)<+\infty .
$$




\subsubsection{Compensated Poisson Random Measures}

Let $\mathrm{M}$ be the jump measure of a càdlàg adapted process and a Poisson random measure relative to $\mathbb{F}$ with intensity measure $\mathrm{m}=\lambda_{+} \otimes \mu$, where $\lambda_{+}$is the Lebesgue measure on $\left(\mathbb{R}_{+}, \mathscr{B}\left(\mathbb{R}_{+}\right)\right)$and $\mu$ a $\sigma$-finite measure on $(\mathbb{R}, \mathscr{B}(\mathbb{R}))$. In this section we define the compensated Poisson random measure and, using the theory of orthogonal measures developed in Gihman \& Skorohod (1974), we introduce the stochastic integral of deterministic functions with respect to a compensated Poisson random measure. First, we introduce the following ring of sets of $E$ :

$$
\mathscr{E}:=\{A \in \mathscr{B}(E): \mathrm{m}(A)<+\infty\} .
$$

To each set $A \in \mathscr{E}$ we associate the random variable $\overline{\mathrm{M}}(A)$ by

$$
\overline{\mathrm{M}}(A):=\mathrm{M}(A)-\mathrm{m}(A), \quad A \in \mathscr{E} .
$$

We remark that $\overline{\mathrm{M}}$ is not defined on $\mathscr{B}(E)$ but only on $\mathscr{E}$. Indeed, on $\mathscr{B}(E)$ expressions of the type " $+\infty-\infty$ " could appear. Notice that $\overline{\mathrm{M}}$ defines a mapping on $\Omega \times \mathscr{E}$ into $(-\infty,+\infty]$ and that $\mathbb{E}[\overline{\mathrm{M}}(A)]=0$ for every $A \in \mathscr{E}$. Now we recall the notion of orthogonal measures (cf. Gihman \& Skorohod (1974), IV§4). Let $\mathscr{K}$ be a semiring of sets of $E$. We assume that to each $A \in \mathscr{K}$ there corresponds a real-valued random variable $\zeta(A)$ with the following properties.

(i) $\zeta(A) \in L^{2}(\mathbb{P})$ and $\zeta(\emptyset)=0$.

(ii) $\zeta(A \cup B)=\zeta(A)+\zeta(B)$ a.s. for disjoint $A$ and $B$ in $\mathscr{K}$.

(iii) $\mathbb{E}[\zeta(A) \zeta(B)]=\alpha(A \cap B)$, where $\alpha$ is a set function on $\mathscr{K}$.

The family $\zeta:=\{\zeta(A), A \in \mathscr{K}\}$ of random variables satisfying the previous three conditions is called an orthogonal random measure and $\alpha(\cdot)$ is called structural function (of $\zeta$ ).

2.2.10 Lemma. The family $\overline{\mathrm{M}}:=\{\overline{\mathrm{M}}(A), A \in \mathscr{E}\}$ of random variables defined by (2.15) is an orthogonal random measure on the ring $\mathscr{E}$ with structural function $\mathrm{m}$.

Proof. It is clear that $\overline{\mathrm{M}}(\emptyset)=0$ and that $\overline{\mathrm{M}}(A \cup B)=\overline{\mathrm{M}}(A)+\overline{\mathrm{M}}(B)$ for disjoint $A$ and $B$ in $\mathscr{E}$. Because of Theorem 2.2.5, the random variable $\mathrm{M}(A)$ is Poisson distributed with parameter $\mathrm{m}(A)$, for every $A$ in $\mathscr{E}$. Therefore, $\mathbb{E}\left[\overline{\mathrm{M}}(A)^{2}\right]$ is the variance of a Poisson-distributed random variable with parameter $\mathrm{m}(A)$, i.e.,

$$
\mathbb{E}\left[\overline{\mathrm{M}}(A)^{2}\right]=\mathbb{E}[\mathrm{M}(A)]=\mathrm{m}(A), \quad A \in \mathscr{E} .
$$

Because of $\mathrm{m}(A)<+\infty$ and of $(2.16)$, we obtain $\overline{\mathrm{M}}(A) \in L^{2}(\mathbb{P}), A \in \mathscr{E}$. It remains to show that $m$ is the structural function of $\bar{M}$. For this aim, we notice that the relation

$$
\overline{\mathrm{M}}(A) \overline{\mathrm{M}}(B)=[\overline{\mathrm{M}}(A \cap B)+\overline{\mathrm{M}}(A \backslash B)] \times[\overline{\mathrm{M}}(A \cap B)+\overline{\mathrm{M}}(B \backslash A)], \quad A, B \in \mathscr{E},
$$

holds. This and (2.16), together with the fact that $\overline{\mathrm{M}}(C)$ and $\overline{\mathrm{M}}(D)$ are independent if $C$ and $D$ are pairwise disjoint sets in $\mathscr{E}$ (cf. Theorem 2.2.5), yield

$$
\begin{aligned}
\mathbb{E}[\overline{\mathrm{M}}(A) \overline{\mathrm{M}}(B)] & =\mathbb{E}\left[\overline{\mathrm{M}}(A \cap B)^{2}\right]+\mathbb{E}[\overline{\mathrm{M}}(A \cap B) \overline{\mathrm{M}}(A \backslash B)] \\
& +\mathbb{E}[\overline{\mathrm{M}}(A \cap B) \overline{\mathrm{M}}(B \backslash A)]+\mathbb{E}[\overline{\mathrm{M}}(A \backslash B) \overline{\mathrm{M}}(B \backslash A)] \\
& =\mathbb{E}\left[\overline{\mathrm{M}}(A \cap B)^{2}\right]=\mathrm{m}(A \cap B), \quad A, B \in \mathscr{E},
\end{aligned}
$$

and the proof is concluded. 
We call the orthogonal random measure $\overline{\mathrm{M}}$ defined by (2.15) on the ring $\mathscr{E}$ the compensated Poisson random measure associated to the random measure $\mathrm{M}$ or simply compensated Poisson random measure. We are going to define the stochastic integral with respect to $\overline{\mathrm{M}}$ for functions in $L^{2}(\mathrm{~m})$. We start defining the stochastic integral for functions belonging to the set $\mathscr{D} \subseteq L^{2}(\mathrm{~m})$ of simple functions:

$$
\mathscr{D}:=\left\{f=\sum_{k=1}^{m} a_{k} 1_{A_{k}}, \quad\left(a_{k}\right)_{k=1}^{m} \subseteq \mathbb{R}, \quad\left(A_{k}\right)_{k=1}^{m} \subseteq \mathscr{E} \text { pairwise disjoint }\right\} .
$$

For $f \in \mathscr{D}$ with the representation

$$
f=\sum_{k=1}^{m} a_{k} 1_{A_{k}}, \quad a_{k} \in \mathbb{R}, \quad A_{k} \in \mathscr{E}, k=1, \ldots, m
$$

we define the elementary stochastic integral with respect to $\bar{M}$, and denote it again by $\overline{\mathrm{M}}$, by

$$
\overline{\mathrm{M}}(f):=\sum_{k=1}^{m} a_{k} \overline{\mathrm{M}}\left(A_{k}\right), \quad f \in \mathscr{D} .
$$

One can verify in a standard way that the elementary stochastic integral with respect to $\overline{\mathrm{M}}$ defines a linear mapping on $\mathscr{D}$ into $L^{2}(\mathbb{P})$ and we omit the proof of this fact. Moreover,

$$
\mathbb{E}[\overline{\mathrm{M}}(f) \overline{\mathrm{M}}(g)]=\mathrm{m}(f g), \quad f, g \in \mathscr{D} .
$$

Because of the linearity of (2.19), it is enough to show (2.20) only for indicator functions. If $f=1_{A}$ and $g=1_{B}$, where $A, B \in \mathscr{E},(2.20)$ is an immediate consequence of Lemma 2.2.10. In particular we get $\overline{\mathrm{M}}(f) \in L^{2}(\mathbb{P})$, for every $f \in \mathscr{D}$. Formula (2.20) can be rephrased saying that (2.19) defines a linear isometric mapping on $\mathscr{D}$ into $L^{2}(\mathbb{P})$. Now we extend the definition of elementary stochastic integral to every function $f \in L^{2}(\mathrm{~m})$ (cf. Gihman \& Skorohod (1974), IV.§4 Theorem 1). We preliminary note that the system $\mathscr{D}$ of simple functions is dense in $L^{2}(\mathrm{~m})$. To see it, we need only to verify that $\mathscr{D}$ satisfies the conditions of Lemma 1.1.8. The set of simple functions is clearly such that $\sigma(\mathscr{D})=\mathscr{B}(E)$. Because $\mathscr{E}$ is a ring, $\mathscr{D}$ is $\cap$-stable and so $\mathscr{D}$ is stable under multiplication. The measure $\mathrm{m}$ is $\sigma$-finite on $E$, hence there exists a sequence $\left(A_{n}\right)_{n \in \mathbb{N}} \subseteq \mathscr{E}$ such that $A_{n} \uparrow E$ as $n \rightarrow+\infty$. Therefore, we can construct a sequence $\left(h_{n}\right)_{n \in \mathbb{N}} \subseteq \mathscr{D}$ converging pointwise to 1 .

2.2.11 Theorem. There exists a unique (continuous) isometric mapping on $L^{2}(\mathrm{~m})$ into $L^{2}(\mathbb{P})$, again denoted by $\overline{\mathrm{M}}$, such that

$$
\overline{\mathrm{M}}\left(1_{B}\right)=\overline{\mathrm{M}}(B), \quad B \in \mathscr{E} .
$$

Proof. For a function $f \in \mathscr{D}$, we have defined the elementary stochastic integral and we know that it is an isometric mapping on $\mathscr{D}$ into $L^{2}(\mathbb{P})$. Moreover, by definition, the elementary stochastic integral satisfies relation (2.21). The linear space $\mathscr{D}$ is dense in $L^{2}(\mathrm{~m})$, hence the elementary stochastic integral with respect to $\overline{\mathrm{M}}$, regarded as a mapping from $\mathscr{D}$ into $L^{2}(\mathbb{P})$, has a unique isometric extension on $L^{2}(\mathrm{~m})$. We denote this extension again by $\overline{\mathrm{M}}$. We need to show the uniqueness of $\overline{\mathrm{M}}$ satisfying 
(2.21). But for this it is enough to observe that any isometric mapping between two Hilbert spaces is continuous. Relation (2.21) defines $\overline{\mathrm{M}}$ uniquely on $\mathscr{D}$ and, by the density of $\mathscr{D}$ in $L^{2}(\mathrm{~m})$ and by continuity, uniquely on $L^{2}(\mathrm{~m})$.

Let $\overline{\mathrm{M}}$ be the unique isometric mapping of Theorem 2.2 .11 and $f \in L^{2}(\mathrm{~m})$. We call $\overline{\mathrm{M}}(f)$ the stochastic integral of $f$ with respect to the compensated Poisson random measure $\overline{\mathrm{M}}$. We can extend the definition of the stochastic integral with respect to $\overline{\mathrm{M}}$ also to functions in $L^{1}(\mathrm{~m})$ as follows. Let $f \in L^{1}(\mathrm{~m})$. We define the stochastic integral of $f$ with respect to $\bar{M}$ by

$$
\overline{\mathrm{M}}(f):=\mathrm{M}(f)-\mathrm{m}(f) .
$$

Of course, from Proposition 2.2.7, the right-hand side of (2.22) is well-defined and finite-valued a.s. It remains to show that it is consistent with the definition of the stochastic integral with respect to $\overline{\mathrm{M}}$.

2.2.12 Proposition. If $f \in L^{1}(\mathrm{~m}) \cap L^{2}(\mathrm{~m})$, then $\mathrm{M}(f)$ and $\overline{\mathrm{M}}(f)$ are both welldefined and

$$
\overline{\mathrm{M}}(f)=\mathrm{M}(f)-\mathrm{m}(f) \quad \text { a.s. }
$$

Proof. By Proposition 2.2.7 and Theorem 2.2.11, the stochastic integrals $\mathrm{M}(f)$ and $\overline{\mathrm{M}}(f)$ are both well-defined and finite-valued a.s. for any $f \in L^{1}(\mathrm{~m}) \cap L^{2}(\mathrm{~m})$. For proving (2.23), in a first step we assume that $f \in L^{1}(\mathrm{~m}) \cap L^{2}(\mathrm{~m})$ is such that $\mathrm{m}(\{f \neq 0\})<+\infty$. Because $\mathscr{D} \subseteq L^{2}(\mathrm{~m})$ is a dense set, there exists a sequence $\left(f_{n}\right)_{n \geq 1} \subseteq \mathscr{D}$ converging to $f$ in $L^{\overline{2}}(\mathrm{~m})$. Replacing, if necessary, $f_{n}$ with $f_{n} 1_{\{f \neq 0\}}$, without loss of generality we can assume that $f_{n}$ vanishes outside of $\{f \neq 0\}$. This implies that $\left(f_{n}\right)_{n \geq 1}$ converges in $L^{1}(\mathrm{~m})$ as well. Relation (2.23), being obviously true for every $f_{n}$, now extends to $f$ by the $L^{2}(\mathrm{~m})$-continuity of $\overline{\mathrm{M}}$ and the $L^{1}(\mathrm{~m})$ continuity of $\mathrm{M}$ and $\mathrm{m}$. (cf. Theorem 2.2 .11 and Lemma 2.2.9). In the case that $f \in L^{1}(\mathrm{~m}) \cap L^{2}(\mathrm{~m})$ is chosen arbitrarily, we define $f_{n}:=f 1_{B_{n}}$ where $B_{n} \in \mathscr{E}$ is such that $B_{n} \uparrow E$ (such a sequence exists because $\mathrm{m}$ is a $\sigma$-finite measure on $(E, \mathscr{B}(E))$ ). Using the theorem of Lebesgue on dominated convergence, we observe that $\left(f_{n}\right)_{n>1}$ converges to $f$ in $L^{1}(\mathrm{~m})$ and $L^{2}(\mathrm{~m})$. Since $\mathrm{m}\left(\left\{f_{n} \neq 0\right\}\right)<+\infty$, we can apply the first step and obtain (2.23) for every $f_{n}$. Again by the continuity property of $\mathrm{M}, \overline{\mathrm{M}}$ and $\mathrm{m}$, we conclude that (2.23) remains valid for $f$.

We conclude this section by giving necessary and sufficient conditions for a $\mathscr{B}(E)$ measurable function $f$ to be integrable with respect to $\overline{\mathrm{M}}$, i.e., to be such that $\overline{\mathrm{M}}(f)$ is well-defined and finite-valued a.s.

2.2.13 Theorem. Let $f$ be a measurable function on $(E, \mathscr{B}(E))$. The integral $\overline{\mathrm{M}}(f)$ exists and is a.s. finite if and only if $\mathrm{m}\left(f^{2} \wedge|f|\right)<+\infty$.

Proof. We first assume that $\mathrm{m}\left(f^{2} \wedge|f|\right)<+\infty$. We have

$$
f=f 1_{\{|f| \leq 1\}}+f 1_{\{|f|>1\}}
$$

with $f 1_{\{|f| \leq 1\}} \in L^{2}(\mathrm{~m})$ and $f 1_{\{|f|>1\}} \in L^{1}(\mathrm{~m})$. By Theorem 2.2.11, $\overline{\mathrm{M}}\left(f 1_{\{|f| \leq 1\}}\right)$ exists and is a.s. finite. By Proposition 2.2.7 and Proposition 2.2.12, $\overline{\mathrm{M}}\left(f 1_{\{|f|>1\}}\right)$ exists, is a.s. finite and consistent. By linearity we put

$$
\overline{\mathrm{M}}(f):=\overline{\mathrm{M}}\left(f 1_{\{|f| \leq 1\}}\right)+\overline{\mathrm{M}}\left(f 1_{\{|f|>1\}}\right) .
$$


Hence, $\overline{\mathrm{M}}(f)$ exists, is a.s. finite and consistent. We do not verify the converse implication and refer to Kallenberg (1997), Theorem 10.15 for a complete proof.

\subsubsection{Construction of Lévy Processes}

In $\S 2.2 .3$ and $\S 2.2 .4$ we have introduced the stochastic integral of deterministic functions with respect to a Poisson random measure and the associated compensated Poisson random measure, respectively. Now we want to apply the developed theory to construct Lévy processes by integration of deterministic functions. We consider a càdlàg process $X$ with jump measure $\mathrm{M}$. We assume that $\mathrm{M}$ is a Poisson random measure relative to the filtration $\mathbb{F}$. The intensity measure of $\mathrm{M}$ is $\mathrm{m}=\lambda_{+} \otimes \nu$, where $\lambda_{+}$is the Lebesgue measure on $\left(\mathbb{R}_{+}, \mathscr{B}\left(\mathbb{R}_{+}\right)\right)$, while $\nu$ is a $\sigma$-finite measure on $(\mathbb{R}, \mathscr{B}(\mathbb{R}))$. We stress that this assumption implies that the process $X$ has no fixed time discontinuities a.s. Furthermore, we require that $\nu$ is a Lévy measure, i.e., $\nu(\{0\})=0$ and $\left(x^{2} \wedge 1\right) \in L^{1}(\nu)$. Notice that the function $h$ defined by $h(t, x):=1_{[0, t]} f(x)$ belongs to $L^{q}\left(\lambda_{+} \otimes \nu\right)$ if and only if the function $f$ belongs to $L^{q}(\nu), q \geq 1$. We recall that, because $\mathbb{F}$ satisfies the usual conditions, every adapted additive process in law relative to $\mathbb{F}$ has an adapted càdlàg modification which is an additive process relative to $\mathbb{F}$ (cf. He, Wang \& Yan (1992), Theorem 2.68). In the sequel we do not distinguish an additive process in law from such a càdlàg modification. We introduce the system of simple functions in $L^{q}(\nu), q \geq 1$, by

$$
\mathscr{D}:=\left\{f=\sum_{j=1}^{m} a_{j} 1_{C_{j}}, a_{j} \in \mathbb{R} ; \quad C_{j} \in \mathscr{B}(\mathbb{R}) \text { p.d., } \quad \nu\left(C_{j}\right)<+\infty\right\},
$$

where the acronym p.d. stands for pairwise disjoint.

2.2.14 Lemma. Let $X$ be a càdlàg adapted process with jump measure $\mathrm{M}$. If $\mathrm{M}$ is a Poisson random measure relative to the filtration $\mathbb{F}$ with intensity measure $\lambda_{+} \otimes \nu$, then for every $f \in \mathscr{D}$ the process $\mathrm{M}\left(1_{[0, \cdot]} f\right)=\left(\mathrm{M}\left(1_{[0, t]} f\right)\right)_{t \geq 0}$ is a Lévy process relative to $\mathbb{F}$.

Proof. Let $f \in \mathscr{D}$ have the representation $f=\sum_{j=1}^{m} a_{j} 1_{C_{j}}$. We put $A_{j}:=\mathbb{R}_{+} \times C_{j}$. For every $t \geq 0$ we have $\mathrm{M}\left(A_{j} \cap[0, t] \times \mathbb{R}\right)<+\infty$ a.s. because $\mathrm{m}\left(A_{j} \cap[0, t] \times \mathbb{R}\right)=$ $\mathrm{m}\left([0, t] \times C_{j}\right)=t \nu\left(C_{j}\right)<+\infty$ and the identity $\mathrm{M}\left(A_{j} \cap[0, t] \times \mathbb{R}\right)=\mathrm{M}\left([0, t] \times C_{j}\right)$, $t \geq 0$, holds. Lemma 2.2.4 ensures that the process $N^{A_{j}}=\left(\mathrm{M}\left([0, t] \times C_{j}\right)\right)_{t \geq 0}$ is a Poisson process relative to $\mathbb{F}$. Therefore the process $\mathrm{M}\left(1_{[0, \cdot]} f\right)$ is càdlàg and adapted because

$$
\mathrm{M}\left(1_{[0, t]} f\right)=\sum_{j=1}^{m} a_{j} \mathrm{M}\left([0, t] \times C_{j}\right) .
$$

Furthermore, $\mathrm{M}\left(1_{\{0\}} f\right)=0$. For $0 \leq s \leq t$, the function $1_{(s, t]} f$ belongs to $L^{1}\left(\lambda_{+} \otimes \nu\right)$ and we can apply Lemma 2.2 .8 to get

$\mathbb{E}\left[\exp \left(i u\left(\mathrm{M}\left(1_{[0, t]} f\right)-\mathrm{M}\left(1_{[0, s]} f\right)\right)\right)\right]=\mathbb{E}\left[\exp \left(i u \mathrm{M}\left(1_{(s, t]} f\right)\right)\right]=\exp \left((t-s) \nu\left(\mathrm{e}^{i u f}-1\right)\right)$. 
This means, in particular, that $\mathrm{M}\left(1_{[0,]} f\right)$ has homogeneous one-dimensional increments. We show the $\mathbb{F}$-independence of the increments. Obviously,

$$
\mathrm{M}\left(1_{[0, t]} f\right)-\mathrm{M}\left(1_{[0, s]} f\right)=\mathrm{M}\left(1_{(s, t]} f\right)=\sum_{j=1}^{m} \mathrm{M}\left((s, t] \times C_{j}\right) .
$$

The sets $(s, t] \times C_{1}, \ldots,(s, t] \times C_{m}$ are pairwise disjoint because $C_{1}, \ldots, C_{m}$ are. Furthermore, $(s, t] \times C_{j} \subseteq(s,+\infty) \times \mathbb{R}$ for every $j=1, \ldots, m$. Hence, the vector $\left(\mathrm{M}\left((s, t] \times C_{1}\right), \ldots, \mathrm{M}\left((s, t] \times C_{m}\right)\right)$ is an independent random vector independent of $\mathscr{F}_{s}$ (cf. Theorem 2.2.5) and so $\mathrm{M}\left(1_{(s, t]} f\right)$ is independent of $\mathscr{F}_{s}$. We have that $\mathrm{M}\left(1_{[0,]} f\right)$ starts at zero, is càdlàg and has homogeneous increments. This is sufficient to assert that it is a stochastically continuous process. Therefore $\mathrm{M}\left(1_{[0, \cdot]} f\right)$ is a Lévy process relative to $\mathbb{F}$ for every $f \in \mathscr{D}$.

As a consequence of Lemma 1.1.8, the system $\mathscr{D}$ introduced in (2.24) is total in $L^{q}(\nu), q \geq 1$. This allows to extend Lemma 2.2.14.

2.2.15 Proposition. Let $X$ be a càdlàg adapted process with jump measure $\mathrm{M}$. If $\mathrm{M}$ is a Poisson random measure relative to the filtration $\mathbb{F}$ with intensity measure $\lambda_{+} \otimes \nu$, then for every $f$ such that $|f| \wedge 1 \in L^{1}(\nu)$ the process $\mathrm{M}\left(1_{[0, \cdot]} f\right)=\left(\mathrm{M}\left(1_{[0, t]} f\right)\right)_{t \geq 0}$ is a Lévy process relative to $\mathbb{F}$.

Proof. First we assume $f \in L^{1}(\nu)$. The system $\mathscr{D}$ is dense in $L^{1}(\nu)$ and so there exists a sequence $\left(f_{n}\right)_{n \in \mathbb{N}} \subseteq \mathscr{D}$ such that $f_{n} \longrightarrow f$ in $L^{1}(\nu)$ as $n \rightarrow+\infty$. By Lemma 2.2 .14 , the sequence $\mathrm{M}\left(1_{[0,]} f_{n}\right)$ is a sequence of Lévy processes relative to $\mathbb{F}$. From the linearity of the stochastic integral and Lemma 2.2.9 we get

$$
\begin{aligned}
0 \leq \mathbb{E}\left[\left|\mathrm{M}\left(1_{[0, t]} f_{n}\right)-\mathrm{M}\left(1_{[0, t]} f\right)\right|\right] & \leq \mathbb{E}\left[\mathrm{M}\left(1_{[0, t]}\left|f_{n}-f\right|\right)\right] \\
& =t \nu\left(\left|f_{n}-f\right|\right) \longrightarrow 0, \quad n \rightarrow+\infty .
\end{aligned}
$$

So we can conclude that $\mathrm{M}\left(1_{[0, t]} f_{n}\right)$ converges to $\mathrm{M}\left(1_{[0, t]} f\right)$ in $L^{1}(\mathbb{P})$. Because the filtration $\mathbb{F}$ satisfies the usual conditions, this implies that the process $\mathrm{M}\left(1_{[0, \cdot]} f\right)$ is $\mathbb{F}$-adapted. Furthermore, the $L^{1}(\mathbb{P})$-convergence of $\mathrm{M}\left(1_{[0, t]} f_{n}\right)$ to $\mathrm{M}\left(1_{[0, t]} f\right)$ implies convergence in probability. By Lemma 2.1.2, $\mathrm{M}\left(1_{[0, \cdot]} f\right)$ is a process with $\mathbb{F}$ independent and homogeneous increments. Obviously, $\mathrm{M}\left(1_{[0, t]} f\right) \longrightarrow 0$ a.s. as $t \downarrow 0$ and from the homogeneity of the increments, we can assert that $\mathrm{M}\left(1_{[0,]} f\right)$ is stochastically continuous (cf. p.34). In conclusion, $\left(\mathrm{M}\left(1_{[0,]} f\right), \mathbb{F}\right)$ is a Lévy process in law. Because the filtration $\mathbb{F}$ satisfies the usual conditions, we can find a version of $\mathrm{M}\left(1_{[0,]} f\right)$ which is in fact a Lévy process relative to $\mathbb{F}$, i.e., also càdlàg. We do not distinguish these two processes and denote the càdlàg version again by $\left(\mathrm{M}\left(1_{[0,]} f\right), \mathbb{F}\right)$. Hence, for every $f \in L^{1}(\mathbb{P})$, the process $\left(\mathrm{M}\left(1_{[0, \cdot]} f\right), \mathbb{F}\right)$ is a Lévy process. We now weaken the assumptions and consider $f$ such that $|f| \wedge 1 \in L^{1}(\nu)$. Because of Proposition 2.2.7, the stochastic integral $\mathrm{M}\left(1_{[0, t]} f\right)$ exists and is finite a.s. for every $t \geq 0$. Denoting by $f^{+}$and $f^{-}$the positive and the negative part of $f$, respectively, we have $f^{ \pm} \leq|f|$ so that $f^{ \pm} \wedge 1 \in L^{1}(\nu)$ and the stochastic integrals $\mathrm{M}\left(1_{[0, t]} f^{ \pm}\right)$are well defined and the relation $\mathrm{M}\left(1_{[0, t]} f\right)=\mathrm{M}\left(1_{[0, t]} f^{+}\right)-\mathrm{M}\left(1_{[0, t]} f^{-}\right)$ holds a.s. Let us introduce the functions $f_{n}^{ \pm}:=f^{ \pm} 1_{\left\{\left|f^{ \pm}\right|<n\right\}}$ and $f_{n}:=f_{n}^{+}-f_{n}^{-}$, $n \geq 1$. Then $f_{n}^{ \pm} \geq 0$ and $f_{n}^{ \pm} \uparrow f^{ \pm}$pointwise as $n \rightarrow+\infty$. Furthermore, $f_{n}^{ \pm} \in L^{1}(\nu)$ 
because $f_{n}^{ \pm} \leq\left(f^{ \pm} \wedge n\right) \leq n\left(f^{ \pm} \wedge 1\right) \in L^{1}(\nu)$. Therefore we also have $f_{n} \in L^{1}(\nu)$. Hence $\mathrm{M}\left(1_{[0, t]} f_{n}^{ \pm}\right)<+\infty$ a.s. $t \geq 0, n \geq 1$, and the theorem of B. Levi on monotone convergence implies that $\mathrm{M}\left(1_{[0, t]} f_{n}^{ \pm}\right) \uparrow \mathrm{M}\left(1_{[0, t]} f^{ \pm}\right)$a.s. as $n \rightarrow+\infty, t \geq 0$. Because of the previous step $\left(\left(\mathrm{M}\left(1_{[0, t]} f_{n}\right)\right)_{t \geq 0}, \mathbb{F}\right)$ is a Lévy process for every $n \geq 1$. Moreover,

$$
\begin{aligned}
\lim _{n \rightarrow+\infty} \mathrm{M}\left(1_{[0, t]} f_{n}\right) & =\lim _{n \rightarrow+\infty} \mathrm{M}\left(1_{[0, t]} f_{n}^{+}\right)-\lim _{n \rightarrow+\infty} \mathrm{M}\left(1_{[0, t]} f_{n}^{-}\right) \\
& =\mathrm{M}\left(1_{[0, t]} f^{+}\right)-\mathrm{M}\left(1_{[0, t]} f^{+}\right) \\
& =\mathrm{M}\left(1_{[0, t]} f\right), \quad t \geq 0 .
\end{aligned}
$$

The previous convergence takes place a.s. and so $\mathrm{M}\left(1_{[0,]} f\right)$ is $\mathbb{F}$-adapted (the filtration satisfies the usual conditions). Furthermore, Lemma 2.1.2 yields $\mathrm{M}\left(1_{[0, \cdot]} f\right)$ has $\mathbb{F}$-independent and homogeneous increments. Clearly, $\mathrm{M}\left(1_{[0, t]} f\right)$ converges to 0 a.s. as $t \downarrow 0$ and we know that this fact, together with the homogeneity of the increments implies that $\mathrm{M}\left(1_{[0, \cdot]} f\right)$ is stochastically continuous. Therefore $\left(\mathrm{M}\left(1_{[0, \cdot]} f\right), \mathbb{F}\right)$ is a Lévy process in law. We do not distinguish such process from its càdlàg modification and so we assert that $\left(\mathrm{M}\left(1_{[0, \cdot]} f\right), \mathbb{F}\right)$ is a Lévy process.

We notice that if $f$ is such that $|f| \wedge 1 \in L^{1}(\nu)$, the process $\mathrm{M}\left(1_{[0, \cdot]} f\right)$ is a semimartingale. Indeed, because of Lemma 2.2.8, we have

$$
\mathbb{E}\left[\exp \left(i u \mathrm{M}\left(1_{[0, t]} f\right)\right)\right]=\exp \left(\mathrm{m}\left(\mathrm{e}^{u i 1_{[0, t]} f}-1\right)\right)=\exp \left(t \nu\left(\mathrm{e}^{u i f}-1\right)\right), \quad t \geq 0 .
$$

Therefore, the function $t \mapsto \mathbb{E}\left[\exp \left(i u \mathrm{M}\left(1_{[0, t]} f\right)\right)\right]$ is of finite variation and the statement follows from Theorem 2.1.7.

Let now $\overline{\mathrm{M}}$ be the compensated Poisson random measure of $L$. From Theorem 2.2.11, every deterministic function in $L^{2}\left(\lambda_{+} \otimes \nu\right)$ can be integrated with respect to $\overline{\mathrm{M}}$. For any $f \in L^{2}(\nu)$ we introduce the process $X^{(f)}=\left(X_{t}^{(f)}\right)_{t \geq 0}$ by

$$
X_{t}^{(f)}=\overline{\mathrm{M}}\left(1_{[0, t]} f\right), \quad t \geq 0 .
$$

These processes will play a key role in the remaining of this thesis and in the following theorem we collect some of their properties. We recall that the spaces $\mathscr{H}_{0}^{2}(\mathbb{F})$ and $\mathscr{H}_{\text {loc }, 0}^{2}(\mathbb{F})$ were introduced in $\S 1.3 .1$.

2.2.16 Theorem. Let $X$ be a càdlàg adapted process with jump measure M. If $\mathrm{M}$ is a Poisson random measure relative to the filtration $\mathbb{F}$ with intensity measure $\lambda_{+} \otimes \nu$ and $\overline{\mathrm{M}}$ is the associated compensated Poisson random measure, then for every $f \in L^{2}(\nu)$ the process $X^{(f)}$ defined by (2.25) has the following properties:

(i) $\mathbb{E}\left[\left(X_{t}^{(f)}\right)^{2}\right]=t \nu\left(f^{2}\right)$ and, in particular, the random variable $X_{t}^{(f)}$ is square integrable, $t \geq 0$;

(ii) $\left(X^{(f)}, \mathbb{F}\right)$ is a Lévy process;

(iii) $X^{(f)}$ belongs to $\mathscr{H}_{\mathrm{loc}, 0}^{2}(\mathbb{F})$ and $\left\langle X^{(f)}, X^{(f)}\right\rangle_{t}=t \nu\left(f^{2}\right), t \geq 0$. Moreover, $X^{(f)^{T}}$ belongs to $\mathscr{H}_{0}^{2}(\mathbb{F})$, for every deterministic time $T$;

(iv) $\Delta X^{(f)}=f(\Delta X) 1_{\{\Delta X \neq 0\}}$ a.s. and, in particular, $X^{(f)}$ is locally bounded if $f$ is bounded;

(v) $X^{(f)}=0$ a.s. if and only if $f=0 \nu$-a.e.;

(vi) Let $f, g \in L^{2}(\nu)$. The martingales $X^{(f)}$ and $X^{(g)}$ are orthogonal if and only if $f$ and $g$ are orthogonal in $L^{2}(\nu)$. 
Proof. (i) is consequence of the isometry of the stochastic integral with respect to $\overline{\mathrm{M}}$ for functions in $L^{2}(\nu)$. We show (ii) and (iii) together. If $f \in L^{2}(\nu)$, then the sequence $f_{n}:=f 1_{\left\{|f|>\frac{1}{n}\right\}}$ belongs to $L^{1}(\nu) \cap L^{2}(\nu)$ and converges to $f$ in $L^{2}(\nu)$ as $n \rightarrow+\infty$. For every $n \geq 1$, Proposition 2.2.12 yields

$$
X_{t}^{\left(f_{n}\right)}=\mathrm{M}\left(1_{[0, t]} f_{n}\right)-t \nu\left(f_{n}\right)=\sum_{0<s \leq t} f_{n}\left(\Delta X_{s}\right) 1_{\left\{\Delta X_{s} \neq 0\right\}}-t \nu\left(f_{n}\right), \quad t \geq 0 .
$$

From Proposition 2.2 .15 we know that $\left(X^{\left(f_{n}\right)}, \mathbb{F}\right)$ is a Lévy process for every $n \geq 1$. Moreover, from (2.26) and Lemma 2.2.9 it follows that $\mathbb{E}\left[X_{t}^{\left(f_{n}\right)}\right]=0, t \geq 0$. Lemma 2.1.1 yields $X^{\left(f_{n}\right)}$ is an $\mathbb{F}$-martingale. We can apply the linearity of the stochastic integral with respect to $\overline{\mathrm{M}}$ and its isometry property for functions in $L^{2}\left(\lambda_{+} \otimes \nu\right)$ to obtain

$$
\mathbb{E}\left[\left(X_{t}^{\left(f_{n}\right)}-X_{t}^{(f)}\right)^{2}\right]=\mathbb{E}\left[\left(X_{t}^{\left(f_{n}-f\right)}\right)^{2}\right]=t \nu\left(\left(f_{n}-f\right)^{2}\right) \longrightarrow 0 \quad \text { as } n \rightarrow+\infty .
$$

Therefore $X_{t}^{\left(f_{n}\right)}$ converges to $X_{t}^{(f)}$ in $L^{2}(\mathbb{P})$, hence in probability, as $n \rightarrow+\infty$. By Lemma 2.1.2 we obtain that $X^{(f)}$ has $\mathbb{F}$-independent and homogeneous increments. The process $X^{\left(f_{n}\right)}$ is $\mathbb{F}$-adapted because it is a Lévy process relative to $\mathbb{F}$, so $(2.27)$ shows that also $X^{(f)}$ is $\mathbb{F}$-adapted, because the filtration $\mathbb{F}$ satisfies the usual conditions. The stochastic continuity of $X^{(f)}$ is clear. Indeed, because of the isometry of the stochastic integral with respect to $\overline{\mathrm{M}}$ for functions in $L^{2}(\nu)$, the mapping $t \mapsto X_{t}^{(f)}$ is continuous as a function of $\mathbb{R}_{+}$into $L^{2}(\mathbb{P})$, hence stochastically continuous. Formula $(2.27)$ implies that $X_{t}^{\left(f_{n}\right)}$ converges in $L^{1}(\mathbb{P})$ to $X_{t}^{(f)}$ and thanks to Lemma 1.2.3 we can conclude that $X^{(f)}$ is an $\mathbb{F}$-martingale. The process $\left(X^{(f)}, \mathbb{F}\right)$ is a Lévy process in law and we consider a version of $X^{(f)}$ which is also càdlàg, i.e., which is in fact a Lévy process relative to $\mathbb{F}$ (cf. He, Wang \& Yan (1992), Theorem 2.68). We denote this modification again by $X^{(f)}:\left(X^{(f)}, \mathbb{F}\right)$ is a Lévy process and an $\mathbb{F}$-martingale. Because of (i), the martingale $X^{(f)}$ belongs to $\mathscr{H}_{\text {loc }, 0}^{2}$ and because of the independence of the increments and (i)

$$
\left\langle X^{(f)}, X^{(f)}\right\rangle_{t}=\mathbb{E}\left[\left(X_{t}^{(f)}\right)^{2}\right]=t \nu\left(f^{2}\right), \quad t \geq 0 .
$$

An application of Doob's inequality implies that for every deterministic stopping time $T>0$, the stopped process $X^{(f)^{T}}$ belongs to $\mathscr{H}_{0}^{2}$, and this concludes the proof of (ii) and (iii). Let $T>0$ be a deterministic stopping time. From (iii), the process $X^{(g)^{T}}$ belongs to $\mathscr{H}_{0}^{2}$, for every $g \in L^{2}(\nu)$. The processes $X^{\left(f_{n}\right)}$ and $X^{(f)}$ are both càdlàg. An application of Doob's inequality and of the linearity and the isometry of the stochastic integral with respect to $\overline{\mathrm{M}}$ for functions in $L^{2}(\nu)$ implies:

$$
\mathbb{E}\left[\sup _{t \in[0, T]}\left(X_{t}^{\left(f_{n}\right)}-X_{t}^{(f)}\right)^{2}\right] \leq 4 \mathbb{E}\left[\left(X_{T}^{\left(f_{n}-f\right)}\right)^{2}\right]=T\left\|f_{n}-f\right\|_{L^{2}(\nu)}^{2} .
$$

We show (iv). By (2.26) we have

$$
\Delta X_{t}^{\left(f_{n}\right)}=f_{n}\left(\Delta X_{t}\right) 1_{\left\{\Delta X_{t} \neq 0\right\}}, \quad t \geq 0, \quad \text { a.s. }
$$

Because $X^{(f)}$ is càdlàg we can define the process $\Delta X^{(f)}$ and by $(2.28)$ we get

$$
\lim _{n \rightarrow+\infty} \Delta X_{t}^{\left(f_{n}\right)}=\Delta X_{t}^{(f)}, \quad t \leq T, \quad \text { a.s. }
$$


From (2.29) we can conclude that $\Delta X^{(f)}=f(\Delta X) 1_{\{\Delta X \neq 0\}}$ a.s. because $T>0$ was chosen arbitrarily. From the relation

$$
X^{(f)}=X_{-}^{(f)}+\Delta X^{(f)}=X_{-}^{(f)}+f(\Delta X) 1_{\{\Delta X \neq 0\}} \quad \text { a.s. }
$$

$X^{(f)}$ is (a.s.) locally bounded if $f$ is bounded (note that $X_{-}^{(f)}$ is locally bounded because $\left.X_{0-}^{(f)}:=X_{0}^{(f)}=0\right)$. Now we come to (v). If $f=0 \nu$-a.e., then it is clear that $X^{(f)}=0$. If $X^{(f)}=0$, from (i), we get $\nu\left(f^{2}\right)=0$ and so $f=0 \nu$-a.e. It remains to verify (vi). Let $f, g \in L^{2}(\nu)$. Using the identity of polarization for $\left\langle X^{(f)}, X^{(g)}\right\rangle$ (cf. (1.5)) and the linearity of $X^{(h)}$ with respect to $h \in L^{2}(\nu)$, we have

$$
\left\langle X^{(f)}, X^{(g)}\right\rangle_{t}=t \nu(f g),
$$

hence $\left\langle X^{(f)}, X^{(g)}\right\rangle=0$ if and only if $\nu(f g)=0$, i.e., if and only if $f$ and $g$ are orthogonal in $L^{2}(\nu)$. The proof of the theorem is now complete.

Obviously, for any $f \in L^{2}(\nu)$, the process $X^{(f)}$ is a semimartingale.

\subsection{Generalities on Lévy Processes}

In $\S 2.3 .1$ we prove that the jump measure of a Lévy process $(L, \mathbb{F})$ is a Poisson random measure relative to the filtration $\mathbb{F}$. In $\$ 2.3 .2$ we recall the Itô-Lévy decomposition and some of its consequences. We do not verify the Itô-Lévy decomposition in this chapter. Rather we postpone its proof to Appendix A. In $\S 2.3 .3$ we discuss the structure of the natural filtration of a Lévy process.

\subsubsection{The Jump Measure of a Lévy Process}

By definition, a Lévy process $(L, \mathbb{F})$ is a càdlàg process. For this reason, the jump measure $\mathrm{M}$ of $L$, given on $(E, \mathscr{B}(E))$ (cf. (2.3)) by (2.5), i.e.,

$$
\mathrm{M}(\omega, A):=\sum_{s \geq 0} 1_{\left\{\Delta L_{s}(\omega) \neq 0\right\}} 1_{A}\left(s, \Delta L_{s}(\omega)\right), \quad A \in \mathscr{B}(E),
$$

is an integer-valued random measure (cf. Proposition 2.2.1). In this section we show that the jump measure of a Lévy process $(L, \mathbb{F})$ is a Poisson random measure relative to the filtration $\mathbb{F}$ with intensity measure $\mathrm{m}:=\lambda_{+} \otimes \nu$, where $\lambda_{+}$is the Lebesgue measure on $\left(\mathbb{R}_{+}, \mathscr{B}\left(\mathbb{R}_{+}\right)\right)$and $\nu$ is a Lévy measure on $(\mathbb{R}, \mathscr{B}(\mathbb{R}))$, i.e., $\nu$ is $\sigma$-finite, such that $\nu(\{0\})=0$ and $\left(x^{2} \wedge 1\right) \in L^{1}(\nu)$. For any Borel subset $B$ of $\mathbb{R}$ contained in $\{|x|>\varepsilon\}$, where $\varepsilon>0$ is arbitrary but fixed, we introduce the process $\xi^{B}=\left(\xi_{t}^{B}\right)_{t \geq 0}$ by

$$
\xi_{t}^{B}:=\mathrm{M}([0, t] \times B), \quad t \geq 0 .
$$

Notice that $\xi^{B}$ can be obtained from (2.6) by choosing $A=\mathbb{R}_{+} \times B$. Because of $B \subseteq\{|x|>\varepsilon\}$ and of the càdlàg property of the paths of $L$, we have that for every $t \geq 0, \mathrm{M}([0, t] \times B)<+\infty$. Therefore, from Lemma $2.2 .2, \xi^{B}$ is an $\mathbb{F}$-adapted simple point process (cf. $\$ 1.2 .4$ ). We are going to show that the process $\xi^{B}$ is a homogeneous Poisson process relative to $\mathbb{F}$. Now the situation is different from the one of Lemma 
2.2.4 because we only know that $\mathrm{M}$ is the jump measure of a Lévy process and not (yet) that it is a Poisson random measure relative to $\mathbb{F}$. We introduce the Skorohod space and the Skorohod $\sigma$-algebra. The space

$$
D:=\left\{z: \mathbb{R}_{+} \longmapsto \mathbb{R} \text { such that the mapping } t \mapsto z(t) \text { is càdlàg }\right\}
$$

of càdlàg functions on $\mathbb{R}_{+}$into $\mathbb{R}$ is called the Skorohod space over $\mathbb{R}$. We consider the mapping $Z_{t}: D \longmapsto \mathbb{R}$ such that $Z_{t}(z):=z(t)$. The $\sigma$-algebra $\mathscr{F}_{D}:=\sigma\left(Z_{t}, t \geq 0\right)$ is called the Skorohod $\sigma$-algebra on $D$. With the Lévy process $(L, \mathbb{F})$ we associate the process ${ }^{t} L$ by

$$
{ }^{t} L_{s}:=L_{t+s}-L_{t}, \quad s \geq 0 .
$$

We now show that the increment $\xi_{t+s}^{B}-\xi_{t}^{B}$ can be represented as a time-homogeneous $\left(D, \mathscr{F}_{D}\right)$-measurable functional of the increment $L_{t+s}-L_{t}, s, t \geq 0$. From this it follows, in particular, that $\xi^{B}$ has $\mathbb{F}$-independent and homogeneous increments.

2.3.1 Lemma. Let $B \in \mathscr{B}(\mathbb{R})$ be such that $B \subseteq\{|x|>\varepsilon\}$, where $\varepsilon>0$ is arbitrary but fixed. We consider the process $\xi^{B}$ introduced by (2.30). For any $s \geq 0$ there exists a measurable functional $F_{s, B}$ over $\left(D, \mathscr{F}_{D}\right)$ such that

$$
\xi_{t+s}^{B}-\xi_{t}^{B}=F_{s, B}\left({ }^{t} L\right) \quad \text { a.s., } \quad t \geq 0 .
$$

Moreover, there exists a constant, say $\nu^{B} \geq 0$, such that

$$
a^{B}(t):=\mathbb{E}\left[\xi_{t}^{B}\right]=t \nu^{B} .
$$

In particular, $\left(\xi^{B}, \mathbb{F}\right)$ is a homogeneous Poisson process.

Proof. We define the functional $G_{s, B}$ by

$$
G_{s, B}(z):=\sum_{0<u \leq s} 1_{\left\{\Delta Z_{u}(z) \neq 0\right\}} 1_{B}\left(\Delta Z_{u}(z)\right), \quad z \in D,
$$

and the sequence $\left(\tau_{k}\right)_{k \geq 0}$ by

$$
\tau_{0}:=0, \quad \tau_{k+1}:=\inf \left\{t>\tau_{k}: \Delta Z_{t}(z) \in B\right\}, \quad k \geq 1 .
$$

Let $\mathbb{Q}$ be a probability measure on $\left(D, \mathscr{F}_{D}\right)$ and $\mathscr{F}_{D}^{\mathbb{Q}}$ the $\mathbb{Q}$-completion of $\mathscr{F}_{D}$. We get that $\tau_{k}$ is $\mathscr{F}_{D}^{\mathbb{Q}}$-measurable. For this we only need to apply Dellacherie (1972), Theorem IV.23, with the filtration $\left(\mathscr{G}_{t}\right)_{t \geq 0}$ defined by $\mathscr{G}_{t}:=\mathscr{F}_{D}^{\mathbb{Q}}$. Then we have that $\left\{z: G_{s, B}(z)=k\right\}=\left\{\tau_{k} \leq t<\tau_{k+1}\right\}$ and $\left\{\tau_{k} \leq t<\tau_{k+1}\right\}$ is $\mathscr{F}_{D}^{\mathbb{Q}}$-measurable. Hence the functional $G_{s, B}$ is measurable on $\left(D, \mathscr{F}_{D}^{\mathbb{Q}}\right)$, for every probability measure $\mathbb{Q}$. Furthermore,

$$
\begin{aligned}
\xi_{t+s}^{B}-\xi_{t}^{B} & =\sum_{t<u \leq t+s} 1_{\left\{\Delta L_{u}(z) \neq 0\right\}} 1_{B}\left(\Delta L_{u}\right) \\
& =\sum_{0<u \leq s} 1_{\left\{\Delta L_{u}(z) \neq 0\right\}} 1_{B}\left(\Delta L_{u}\right)=G_{s, B}\left({ }^{t} L\right), \quad t, s \geq 0 .
\end{aligned}
$$

If we now choose $\mathbb{Q}:=\mathbb{P}_{L}=\mathbb{P}_{t_{L}}$ we find $F_{s, B}$ on $\left(D, \mathscr{F}_{D}\right)$ such that $F_{s, B}=G_{s, B} \mathbb{P}_{L^{-}}$ a.s. (where $\mathbb{P}_{L}$ denotes the law of $L$ ). From Lemma 2.2.2, we know that $\xi^{B}$ is a simple point process relative to $\mathbb{F}$. We can assert that $\xi^{B}$ is a simple point process with 
$\mathbb{F}$-independent and homogeneous increments. Because $\xi^{B}$ is càdlàg and starts at 0, the homogeneity of the increments implies that $\xi^{B}$ is also stochastically continuous, i.e., $\left(\xi^{B}, \mathbb{F}\right)$ is a Lévy process with bounded jumps. Because of Proposition 2.1.6, the process $\xi^{B}$ has a finite moment of every order. We can introduce the process $\bar{\xi}^{B}=\left(\bar{\xi}_{t}^{B}\right)_{t \geq 0}$ by $\bar{\xi}_{t}^{B}:=\xi_{t}^{B}-\mathbb{E}\left[\xi_{t}^{B}\right]$. Because of Lemma 2.1.1, $\bar{\xi}^{B}$ is an $\mathbb{F}$-martingale, hence $\left|\bar{\xi}^{B}\right|$ is a submartingale. If $T>0$ is arbitrary but fixed, we get $\bar{\xi}_{T}^{B} \in L^{1}(\mathbb{P})$ and

$$
\left|\bar{\xi}_{t}^{B}\right| \leq \mathbb{E}\left[\left|\bar{\xi}_{T}^{B}\right| \mid \mathscr{F}_{t}\right], \quad 0 \leq t \leq T .
$$

From the previous estimate and He, Wang \& Yan (1992), Theorem 1.8, $\left(\left|\bar{\xi}_{t}^{B}\right|\right)_{0 \leq t \leq T}$ is uniformly integrable and hence $\left(\xi_{t}^{B}\right)_{0 \leq t \leq T}$ is uniformly integrable. We define $a^{B}(t):=\mathbb{E}\left[\xi_{t}^{B}\right], t \geq 0$. Because of the uniform integrability, we can apply the generalization of the theorem of Lebesgue on dominated convergence to uniformly integrable families (cf. Meyer (1966), Theorem II.21) to obtain that $a^{B}(\cdot)$ is a continuous function. Moreover, because of the homogeneity of the increments, we have

$$
a^{B}(t+s)=\mathbb{E}\left[\xi_{t+s}^{B}-\xi_{t}^{B}\right]+\mathbb{E}\left[\xi_{t}^{B}\right]=a^{B}(s)+a^{B}(t), \quad t, s \geq 0 .
$$

This relation together with the continuity, implies that the function $a^{B}(\cdot)$ is linear and therefore that there exists a $\nu^{B} \geq 0$ such that $a^{B}(t)=t \nu^{B}$. In conclusion $\xi^{B}$ is a stochastically continuous simple point process with $\mathbb{F}$-independent and homogeneous increments such that $\mathbb{E}\left[\xi_{t}^{B}\right]=t \nu^{B}, t \geq 0, \nu^{B} \geq 0$, i.e., $\left(\xi^{B}, \mathbb{F}\right)$ is a homogeneous Poisson process (cf. Definition 1.2.6) and the proof of the lemma is complete.

Thanks to Lemma 2.3.1, we can compute the explicit form of the intensity measure of the random measure $\mathrm{M}$. For any $C \in \mathscr{B}(\mathbb{R})$, we put

$$
\nu(C):=\mathbb{E}[\mathrm{M}([0,1] \times C)] .
$$

Clearly, (2.34) defines a measure on the space $(\mathbb{R}, \mathscr{B}(\mathbb{R}))$. From the definition of $\mathrm{M}$, we have that $\nu(\{0\})=0$. Moreover, $\nu(\{|x|>\varepsilon\})<+\infty$, for every $\varepsilon>0$. Indeed, because of Lemma 2.3.1, the process $\left(\xi^{B}, \mathbb{F}\right)$, where $B:=\{|x|>\varepsilon\}$, is a homogeneous Poisson process and therefore

$$
\nu(B)=\mathbb{E}[\mathrm{M}([0,1] \times B)]=\mathbb{E}\left[\xi_{1}^{B}\right]=\nu^{B}<+\infty,
$$

where the constant $\nu^{B} \geq 0$ was introduced in (2.33). This implies that $\nu$ is a $\sigma$-finite measure on $(\mathbb{R}, \mathscr{B}(\mathbb{R}))$ because the sequence $\left(B_{n}\right)_{n \geq 1}$ defined by $B_{n}:=\left\{|x|>\frac{1}{n}\right\}$, $n \geq 1$, is such that $B_{n} \uparrow \mathbb{R} \backslash\{0\}$ as $n \rightarrow+\infty$ and $\nu(\mathbb{R})=\nu(\mathbb{R} \backslash\{0\})$. It can be proven that the function $\left(x^{2} \wedge 1\right)$ is integrable with respect to $\nu$. We do not verify this property and we refer to, e.g., Kallenberg (1997), Theorem 13.4. In conclusion, $\nu$ is a Lévy measure. We call $\nu$ the Lévy measure of the process $L$.

2.3.2 Lemma. Let $\mathrm{M}$ be the jump measure of the Lévy process $(L, \mathbb{F})$ with intensity measure $\mathrm{m}$. Then $\mathrm{m}=\lambda_{+} \otimes \nu$, where $\lambda_{+}$is the Lebesgue measure on $\left(\mathbb{R}_{+}, \mathscr{B}\left(\mathbb{R}_{+}\right)\right)$ and $\nu$ is the Lévy measure of $L$.

Proof. Because of Lemma 2.3.1, we have that $\mathrm{m}(A)=\left(\lambda_{+} \otimes \nu\right)(A)$ if $A \in E$ is such that $A=[0, u] \times\{|x|>\varepsilon\}, \varepsilon>0, u \geq 0$. To obtain that $\mathrm{m}=\lambda_{+} \otimes \nu$ is 
now a standard procedure with the help of the uniqueness theorem for measures (cf. Theorem 1.1.2). We introduce the system of sets

$$
\begin{aligned}
\mathscr{C}:=\{\{0\} \times C, & C \in \mathscr{B}(\mathbb{R}): \nu(C)<+\infty\} \cup \\
& \cup\{(r, v] \times C: 0 \leq r \leq v, C \in \mathscr{B}(\mathbb{R}): \nu(C)<+\infty\}
\end{aligned}
$$

which generates $\mathscr{B}(E)$ and is stable under intersections. If $A=(r, v] \times B$ with $0<r<v$ and $B \in \mathscr{B}(\mathbb{R})$ is such that $B \subseteq\{|x|>\varepsilon\}, \varepsilon>0$, then $A \in \mathscr{C}$ and $\mathrm{M}(A)=\mathrm{M}([0, v] \times B)-\mathrm{M}([0, r] \times B)=\xi_{v}^{B}-\xi_{r}^{B}$. From the previous step, this yields $\mathrm{m}(A)=\mathrm{m}([0, v] \times B)-\mathrm{m}([0, r] \times B)=(v-r) \nu(B)=\left(\lambda_{+} \otimes \nu\right)(A)$. The sets $A_{n}:=[0, n] \times\left(\left\{|x|>\frac{1}{n}\right\} \cup\{0\}\right), n \geq 1$, belong to $\mathscr{C}$ and are such that $\cup_{n=1}^{\infty} A_{n}=E$. Hence $\left(A_{n}\right)_{n \geq 1}$ is a sequence of sets of finite measure with respect to both $\mathrm{m}$ and $\lambda_{+} \otimes \nu$. An application of the uniqueness theorem for measures (cf. Theorem 1.1.2) shows that $\mathrm{m}=\lambda_{+} \otimes \nu$ on $\mathscr{B}(E)$.

Now we show that, for every $A \in \mathscr{B}(E)$ such that $\mathrm{m}(A)<+\infty$, the process $\left(N^{A}, \mathbb{F}\right)$ defined by $(2.6)$ is a Poisson process.

2.3.3 Proposition. Let $\mathrm{M}$ be the jump measure of a Lévy process $(L, \mathbb{F})$ with intensity measure $\mathrm{m}=\lambda_{+} \otimes \nu$ and let $A \in \mathscr{B}(E)$ be such that $\left(\lambda_{+} \otimes \nu\right)(A)<+\infty$. Then the process $N^{A}:=\left(N_{t}^{A}\right)_{t \geq 0}$ defined by $N_{t}^{A}:=\mathrm{M}(A \cap[0, t] \times \mathbb{R}), t \geq 0$, is a Poisson process relative to $\mathbb{F}$ and $a^{A}(\cdot):=\left(\lambda_{+} \otimes \nu\right)(A \cap[0, \cdot] \times \mathbb{R})$ is its intensity function.

Proof. The random measure $\mathrm{M}$ is the jump measure of the càdlàg process $L$ and therefore, thanks to Lemma 2.2.2 and the comment following it, we know that $N^{A}$ is an $\mathbb{F}$-adapted simple point process. Because of the definition of $N^{A}$ and Lemma 2.3.2, we have $\mathbb{E}\left[N_{t}^{A}\right]=\left(\lambda_{+} \otimes \nu\right)(A \cap[0, t] \times \mathbb{R})<+\infty, t \geq 0$, which is a continuous function of time. To conclude that $\left(N^{A}, \mathbb{F}\right)$ is a Poisson process, we need only to prove that it has $\mathbb{F}$-independent increments. Let $B$ be a Borel subset of $\{|x|>\varepsilon\}$, $\varepsilon>0$. Because of Lemma 2.3.1 $\left(\xi^{B}, \mathbb{F}\right)$ is an homogeneous Poisson process. If now $A \in \mathscr{B}(E)$ is of the form $A=(r, v] \times B, 0<r<v$ and $B$ Borel subset of $\{|x|>\varepsilon\}$, $\varepsilon>0$, we get

$$
N^{A}=\left(\xi^{B}\right)^{v}-\left(\xi^{B}\right)^{r},
$$

where the superscripts $v$ and $r$ denote the operation of stopping at the deterministic time $v$ and $r$, respectively. From Lemma A.1.6, the process $N^{A}$ has $\mathbb{F}$-independent increments and so it is a Poisson process relative to $\mathbb{F}$. If now we take $A_{1}, \ldots, A_{m}$, $m \geq 1$, pairwise disjoint subsets of $E$ such that $A_{j}=\left(r_{j}, v_{j}\right] \times B_{j}$, where $0<r_{j}<v_{j}$ and $B_{j}$ is a Borel subset of $\{|x|>\varepsilon\}, \varepsilon>0, j=1, \ldots, m$, from the previous step, we have that $N^{A_{1}}, \ldots, N^{A_{m}}$ are Poisson processes relative to $\mathbb{F}$ and moreover

$$
\begin{aligned}
{\left[N^{A_{j}}, N^{A_{k}}\right]_{t} } & =\sum_{0<s \leq t} \Delta N_{s}^{A_{j}} \Delta N_{s}^{A_{k}} \\
& =\sum_{0<s \leq t} \Delta \mathrm{M}\left(A_{j} \cap[0, s] \times \mathbb{R}\right) \Delta \mathrm{M}\left(A_{k} \cap[0, s] \times \mathbb{R}\right) \\
& =\sum_{0<s \leq t} 1_{\left\{\Delta X_{s} \neq 0\right\}} 1_{A_{j} \cap A_{k}}\left(s, \Delta X_{s}\right)=0, \quad j, k=1, \ldots, m, \quad j \neq k .
\end{aligned} .
$$

Therefore, from Theorem 2.1.8, the vector $\left(N^{A_{1}}, \ldots, N^{A_{m}}\right)$ has $\mathbb{F}$-independent in- 
crements. We fix $T>0$ and define the algebra of subsets $\mathscr{C} \subseteq \mathscr{B}(E)$ as a such:

$$
\begin{aligned}
& \mathscr{C}:=\left\{\bigcup_{j=1}^{n} A_{j}, \quad A_{j}:=\left(r_{j}, v_{j}\right] \times B_{j} \text { p.d., } \quad 0 \leq r \leq v \leq T,\right. \\
& \left.B_{j} \in \mathscr{B}(\mathbb{R}): B_{j} \subseteq\{|x|>\varepsilon\}\right\},
\end{aligned}
$$

where the acronym p.d. stands for pairwise disjoint. For $A \in \mathscr{C}$ we have

$$
N_{t}^{A}-N_{s}^{A}=\sum_{j=1}^{n}\left(N_{t}^{A_{j}}-N_{s}^{A_{j}}\right) .
$$

Because of the previous step, $\left(N_{t}^{A_{1}}-N_{s}^{A_{1}}, \ldots, N_{t}^{A_{m}}-N_{s}^{A_{m}}\right)$ is an $\mathscr{F}_{s}$-independent vector and therefore we can conclude that $N^{A}$ has $\mathbb{F}$-independent increments, for every $A \in \mathscr{C}$. We now define the system of sets $\mathscr{A} \subseteq \mathscr{B}(E)$ by

$$
\mathscr{A}:=\left\{A \in \mathscr{B}(E), A \subseteq[0, T) \times\{|x|>\varepsilon\}: \quad N^{A} \text { has } \mathbb{F} \text {-independent increments }\right\} .
$$

It is clear that $\mathscr{A}$ is a monotone class of subsets of $\mathscr{B}(E)$ (cf. $\S 1.1 .2)$. From the previous step, $\mathscr{C} \subseteq \mathscr{A}$ and moreover $\sigma(\mathscr{C})=\mathscr{B}([0, T)) \otimes \mathscr{B}(\{|x|>\varepsilon\})$. An application of the monotone class theorem for systems of sets (cf. Theorem 1.1.5), yields $\mathscr{A}=\mathscr{B}([0, T)) \otimes \mathscr{B}(\{|x|>\varepsilon\})$. We now consider the general case in which $A \in \mathscr{B}(E)$ is a set such that $\mathrm{m}(A)<+\infty$. We define the sequence $\left(A_{n}\right)_{n \geq 1}$ by

$$
A_{n}:=A \cap[0, n) \times\left\{|x|>\frac{1}{n}\right\}, \quad n \geq 1 .
$$

From the previous step we know that $N^{A_{n}}$ has $\mathbb{F}$-independent increments. On the other side, we have

$$
\left(N_{t}^{A}-N_{s}^{A}\right)=\lim _{n \rightarrow+\infty}\left(N_{t}^{A_{n}}-N_{s}^{A_{n}}\right), \quad 0 \leq s \leq t,
$$

and the previous convergence takes place pointwise in $\omega$. Therefore we can conclude that $\left(N_{t}^{A}-N_{s}^{A}\right)$ is independent of $\mathscr{F}_{s}, 0 \leq s \leq t$, and the proof is complete.

It is now immediate to see that if $\mathrm{M}$ is the jump measure of a Lévy process $(L, \mathbb{F})$, then it is a Poisson random measure relative to the filtration $\mathbb{F}$. Indeed, if we fix $s>0$ and we consider a Borel subset $A$ of $(s,+\infty) \times \mathbb{R}$ such that $\mathrm{m}(A)<+\infty$, because of Proposition 2.3.3, the process $\left(N^{A}, \mathbb{F}\right)$ is a Poisson process. Notice that for such a choice of $A$, we have that $N_{s}^{A}=0$. Therefore

$$
\mathrm{M}(A)=N_{\infty}^{A}=N_{\infty}^{A}-N_{s}^{A}=\lim _{t \rightarrow+\infty}\left(N_{t}^{A}-N_{s}^{A}\right),
$$

where the previous limit is meant pointwise in $\omega$. Because of Proposition 2.3.3, $\left(N_{t}^{A}-N_{s}^{A}\right)$ is independent of $\mathscr{F}_{s}$, hence $\mathrm{M}(A)$ is independent of $\mathscr{F}_{s}$. Lemma 2.3.2 implies that the intensity measure $\mathrm{m}$ of $\mathrm{M}$ is equal to $\lambda_{+} \otimes \nu$. In conclusion we have shown the following result: 
2.3.4 Theorem. Let $(L, \mathbb{F})$ be a Lévy process and let $\mathrm{M}$ be its jump measure. Then $\mathrm{M}$ is a Poisson random measure relative to the filtration $\mathbb{F}$ and its intensity measure is given by $\mathrm{m}=\lambda_{+} \otimes \nu$, where $\lambda_{+}$is the Lebesgue measure on $\left(\mathbb{R}_{+}, \mathscr{B}\left(\mathbb{R}_{+}\right)\right)$and $\nu$ is the Lévy measure of the process $L$.

From now on, we call the jump measure of a Lévy process $(L, \mathbb{F})$ the Poisson random measure of $L$ and the associated compensated Poisson random measure $\overline{\mathrm{M}}$ the compensated Poisson random measure of $L$.

\subsubsection{Itô-Lévy Decomposition and Moments}

In this section we consider a càdlàg adapted process $L$ and we characterize the structure that it must have to be a Lévy process relative to the filtration $\mathbb{F}$. The main result of this part is the well-known Itô-Lévy decomposition. We state the ItôLévy decomposition for Lévy processes relative to a general filtration $\mathbb{F}$ satisfying the usual conditions. Proofs of the Itô-Lévy decomposition for Lévy processes relative to a filtration can be found, e.g., in He, Wang \& Yan (1992) or Jacod \& Shiryaev (2000) but they make use of the general theory of semimartingales and, in particular, of the decomposition of a semimartingale in its characteristics. We would like to give a more elementary proof which does not use the general theory of semimartingales. Some further preparation is necessary however and for this reason we postpone the proof to Appendix A.1. Here we only formulate the Itô-Lévy decomposition in the form which we need. In other books, like Kallenberg (1997) or Sato (1999) the ItôLévy decomposition is established only for Lévy processes and not for Lévy processes relative to a filtration.

2.3.5 Theorem (Itô-Lévy decomposition). Let $L$ be a càdlàg adapted process with jump measure $\mathrm{M}$. Then $(L, \mathbb{F})$ is a Lévy process if and only if $\mathrm{M}$ is a Poisson random measure relative to $\mathbb{F}$ with intensity function $\lambda_{+} \otimes \nu$, where $\lambda_{+}$is the Lebesgue measure on $\mathbb{R}_{+}$and $\nu$ a Lévy measure, and there exists a Wiener process $\left(\mathrm{W}^{\sigma}, \mathbb{F}\right)$ with variance function $\sigma^{2}(t)=\sigma^{2} t, \sigma^{2} \geq 0$, called Gaussian part of $L$, such that the following decomposition holds

$$
L_{t}=\beta t+\mathrm{W}_{t}^{\sigma}+\mathrm{M}\left(1_{[0, t] \times\{|x|>1\}} x\right)+\overline{\mathrm{M}}\left(1_{[0, t] \times\{|x| \leq 1\}} x\right), \quad t \geq 0, \text { a.s. },
$$

where $\beta \in \mathbb{R}$.

Let $(L, \mathbb{F})$ be a Lévy process with Itô-Lévy decomposition as in (2.35). We call the triplet $\left(\beta, \sigma^{2}, \nu\right)$ appearing in (2.35) the characteristic triplet or simply the characteristics of $L$. If $\sigma^{2}=0$ we say that $L$ is a purely non-Gaussian Lévy process.

Notice that in the usual formulation of the Itô-Lévy decomposition, the independence of the terms appearing in (2.35) is required. However this is not needed, because it is automatically guaranteed by Theorem 2.1.8. Let us discuss this fact. We assume that $\mathrm{M}$ is the jump measure of a càdlàg process and that it is a Poisson random measure relative to $\mathbb{F}$ with intensity measure $\lambda_{+} \otimes \nu$. Furthermore, let $(W, \mathbb{F})$ be a Wiener process. We define the vector $\left(L^{1}, L^{2}, L^{3}\right)$ by

$$
\left(L^{1}, L^{2}, L^{3}\right):=\left(W, \mathrm{M}\left(1_{[0, \cdot] \times\{|x|>1\}} x\right), \overline{\mathrm{M}}\left(1_{[0, \cdot] \times\{|x| \leq 1\}} x\right)\right) .
$$


From $\S 2.2 .5$, we know that $\left(L^{2}, \mathbb{F}\right)$ and $\left(L^{3}, \mathbb{F}\right)$ are Lévy processes (and semimartingales). More precisely, they are purely non-Gaussian Lévy processes with no common jumps, so $\left[L^{2}, L^{3}\right]=0$. Because $\left(L^{1}, \mathbb{F}\right)$ is a Wiener process, it is a continuous martingale and this yields $\left[L^{1}, L^{j}\right]=0, j=2,3$. From Theorem 2.1.8 we can conclude that the vector $\left(L^{1}, L^{2}, L^{3}\right)$ is independent. We can even state more: M and $W$ are independent. Indeed, if $A_{1}, \ldots, A_{m}$ are pairwise disjoint Borel subsets of $E$ such that $\mathrm{m}\left(A_{j}\right)<+\infty, j=1, \ldots,+\infty$, from Lemma 2.2.4, we know that $\left(N^{A_{j}}, \mathbb{F}\right)$ is a Poisson processes, $j=1, \ldots, m$. Moreover, $\left[N^{A_{j}}, N^{A_{k}}\right]=0, j \neq k$, and $\left[W, N^{A_{j}}\right]=0, j=1, \ldots, m$. From Theorem 2.1.8, $\left(W, N^{A_{1}}, \ldots, N^{A_{m}}\right)$ is an independent vector and therefore $\left(W, \mathrm{M}\left(A_{1}\right), \ldots, \mathrm{M}\left(A_{m}\right)\right)=\left(W, N_{\infty}^{A_{1}}, \ldots, N_{\infty}^{A_{m}}\right)$ is independent. If $A_{1}, \ldots, A_{m}$ are not pairwise disjoint we can reduce the situation to the previous case by considering an appropriate partition of the union of the $A_{j} \mathrm{~s}$, and obtain that for every $A_{1}, \ldots, A_{m}$ Borel subsets of $E$ such that $\mathrm{m}\left(A_{j}\right)<+\infty$, the vector $\left(\mathrm{M}\left(A_{1}\right), \ldots, \mathrm{M}\left(A_{m}\right)\right)$ is independent of $W$, which issues the independence of $W$ and $\mathrm{M}$.

The characteristic triplet of a Lévy process characterizes the probability measure $\mathbb{P}$ on the $\sigma$-field $\tilde{\mathscr{F}}_{\infty}^{L}$ (cf. Example 1.2.1):

2.3.6 Theorem. Let $(L, \mathbb{F})$ be a Lévy process with respect to two probability measures $\mathbb{P}$ and $\mathbb{Q}$ on $\mathscr{F}$. If $L$ has the same characteristic triplet $\left(\beta, \sigma^{2}, \nu\right)$ both with respect to $\mathbb{P}$ and $\mathbb{Q}$, then $\left.\mathbb{P}\right|_{\tilde{\mathscr{F}}_{\infty}^{L}}=\left.\mathbb{Q}\right|_{\tilde{F}_{\infty}^{L}}$.

Proof. cf. Jacod \& Shiryaev (2000), Theorem II.4.25.

An immediate but important consequence of Theorem 2.3.5 is the Lévy-Khintchine decomposition. We postpone the proof to Appendix A.1.

2.3.7 Corollary. Let $L$ be a Lévy process with characteristics $\left(\beta, \sigma^{2}, \nu\right)$. Then for every $u \in \mathbb{R}$ and for every $t \geq 0$ we have

$$
\mathbb{E}\left[\mathrm{e}^{i u L_{t}}\right]=\exp \left(\left(i u \beta-\frac{1}{2} u^{2} \sigma^{2}+\int_{\mathbb{R}}\left(\mathrm{e}^{i u x}-1-i u x 1_{\{|x| \leq 1\}}\right) \mathrm{d} \nu\right) t\right) .
$$

We notice that (2.36) implies that the mapping $t \mapsto \mathbb{E}\left[\mathrm{e}^{i u L_{t}}\right], t \geq 0$, is of finite variation for every $u \in \mathbb{R}$. Therefore, from Theorem 2.1.7, we can deduce that every Lévy process is a semimartingale. We conclude this part stating a result which explains in terms of the Lévy measure when a Lévy process is of finite variation and when its moments exist and are finite.

2.3.8 Theorem. Let $(L, \mathbb{F})$ be a Lévy process with Lévy measure $\nu$.

(i) $L$ is of finite variation if and only if $(|x| \wedge 1) \in L^{1}(\nu)$.

(ii) Let $n \in \mathbb{N}$. Then $\mathbb{E}\left[\left|L_{t}\right|^{n}\right]<+\infty, t \geq 0$, if and only if $1_{\{|x|>1\}} x^{n} \in L^{1}(\nu)$.

(iii) Let $c>0$. Then $\mathbb{E}\left[\mathrm{e}^{c\left|L_{t}\right|}\right]<+\infty, t \geq 0$, if and only if $1_{\{|x|>1\}} \mathrm{e}^{c|x|} \in L^{1}(\nu)$.

(iv) If $L$ is a Lévy process with bounded jumps then it admits exponential moments of every order $c \geq 0$.

Proof. cf. Sato (1999), §25. 


\subsubsection{The Structure of the Natural Filtration of a Lévy Process}

Let $L$ be a Lévy process on the probability space $\left(\Omega, \mathscr{F}_{\infty}^{L}, \mathbb{P}\right)$ and $\mathbb{F}^{L}$ the completion in $\mathscr{F}_{\infty}^{L}$ of the filtration generated by $L$. Because of Proposition 2.1.4 $L$ is a Lévy process relative to $\mathbb{F}^{L}$ and $\mathbb{F}^{L}$ coincides with the natural filtration of $L$. We denote by M the jump measure of $L$ and by $\nu$ its Lévy measure. The Itô-Lévy decomposition of $L$ is

$$
L_{t}=\beta t+\mathrm{W}_{t}^{\sigma}+\mathrm{M}\left(1_{[0, t] \times\{|x|>1\}} x\right)+\overline{\mathrm{M}}\left(1_{[0, t] \times\{|x| \leq 1\}} x\right), \quad t \geq 0, \text { a.s. }
$$

By $\tilde{\mathscr{F}}_{t}^{\mathrm{M}}:=\sigma(\mathrm{M}(A), A \in \mathscr{B}(E), A \subseteq[0, t] \times \mathbb{R})$, we define $\tilde{\mathbb{F}}^{\mathrm{M}}=\left(\tilde{\mathscr{F}}_{t}^{\mathrm{M}}\right)_{t \geq 0}$ and we call it the filtration generated by $\mathrm{M}$. The space $(E, \mathscr{B}(E))$ was introduced in (2.3). By $\mathbb{F}^{\mathrm{M}}$ we denote the $\mathbb{P}$-completion of $\tilde{\mathbb{F}}^{\mathrm{M}}$ in $\mathscr{F}_{\infty}^{L}$. The terminal $\sigma$-algebra of $\tilde{\mathbb{F}}^{\mathrm{M}}$ is $\tilde{\mathscr{F}}_{\infty}^{\mathrm{M}}:=\sigma(\mathrm{M}(A), A \in \mathscr{B}(E))$ and $\mathscr{F}_{\infty}^{\mathrm{M}}$ designates its $\mathbb{P}$-completion in $\mathscr{F}_{\infty}^{L}$. Now we give a generator of the $\sigma$-algebra $\tilde{\mathscr{F}}_{t}^{M}, t \geq 0$, and one of the $\sigma$-algebra $\tilde{\mathscr{F}}_{\infty}^{\mathrm{M}}$. First we introduce the subsystems $\mathscr{C}$ and $\mathscr{C}_{t}$ of $\mathscr{B}(E)$ by

$$
\begin{aligned}
& \mathscr{C}:=\{(u, v] \times(a, b], u, v \geq 0,0 \notin[a, b]\}, \\
& \mathscr{C}_{t}:=\{(u, v] \times(a, b], 0 \leq u \leq v \leq t, 0 \notin[a, b]\} .
\end{aligned}
$$

2.3.9 Lemma. The system $\mathscr{C}^{\mathrm{M}}:=\{\mathrm{M}(A), A \in \mathscr{C}\}$ generates the $\sigma$-algebra $\tilde{\mathscr{F}}_{\infty}^{\mathrm{M}}$, while $\mathscr{C}_{t}^{\mathrm{M}}:=\left\{\mathrm{M}(A), A \in \mathscr{C}_{t}\right\}$ generates $\tilde{\mathscr{F}}_{t}^{\mathrm{M}}, t \geq 0$.

Proof. We show only that $\mathscr{C}^{\mathrm{M}}$ generates $\tilde{\mathscr{F}}_{\infty}^{\mathrm{M}}$. The proof that $\mathscr{C}_{t}^{\mathrm{M}}$ generates $\tilde{\mathscr{F}}_{t}^{\mathrm{M}}$ is analogous. From the definition of $\tilde{\mathscr{F}}_{\infty}^{\mathrm{M}}$, the inclusion $\sigma\left(\mathscr{C}_{\mathrm{M}}\right) \subseteq \tilde{\mathscr{F}}_{\infty}^{\mathrm{M}}$ is evident. The converse implication is an easy application of the theorem on Dynkin systems (cf. Bauer (2001), I§2). The family $\mathscr{C}$ is an $\cap$-stable generator of $\mathscr{B}(E)$. If $A \in \mathscr{B}(E)$, we can define the sequence $A_{n}:=A \cap[0, n) \times\left\{\frac{1}{n}<|x| \leq n\right\}, n \geq 1$, and we have $\mathrm{M}\left(A_{n}\right) \uparrow \mathrm{M}(A)$ pointwise in $\omega$ as $n \rightarrow+\infty$. Therefore, it is enough to show that $\mathrm{M}\left(A_{n}\right)$ is $\sigma\left(\mathscr{C}_{\mathrm{M}}\right)$-measurable, for every $n \geq 1$. We fix $n \geq 1$. The system $\mathscr{K}:=\left\{A \in \mathscr{B}(E): \mathrm{M}\left(A_{n}\right)\right.$ is $\sigma\left(\mathscr{C}_{\mathrm{M}}\right)$-measurable $\}$ is a Dynkin system. Indeed, $\mathbb{R}_{+} \times \mathbb{R}$ belongs to $\mathscr{K}$. From this, it is immediate to get that if $A \in \mathscr{K}$, then $A^{c} \in \mathscr{K}$. Moreover, countable unions of pairwise disjoint elements of $\mathscr{K}$ belong again to it. We also have $\mathscr{C} \subseteq \mathscr{K}$. Because of the theorem on Dynkin systems (cf. Bauer (2001), Theorem I.2.4), we have $\mathscr{K}=\mathscr{B}(E)$.

Let $\mathscr{D}$ be the system of simple functions of $L^{q}(\nu), q \geq 1$, defined in (2.24). The system $\mathscr{D}$ is total in $L^{q}(\nu), q \geq 1$ (cf. Lemma 1.1.8). By definition of $\tilde{\mathscr{F}}_{t}^{\mathrm{M}}$, the process $\mathrm{M}\left(1_{[0, \cdot]} f\right)$ is $\tilde{\mathbb{F}}^{\mathrm{M}}$-adapted, for every $f \in \mathscr{D}$. The proof of following proposition is similar to the one of Proposition 2.2.15 and Theorem 2.2.16 and we omit it.

2.3.10 Proposition. Let $\mathrm{M}$ be the jump measure of $L$. If $|f| \wedge 1 \in L^{1}(\nu)$, then $\mathrm{M}\left(1_{[0, \cdot]} f\right)$ is $\mathbb{F}^{\mathrm{M}}$-adapted and if $f \in L^{2}(\nu)$, then $X^{(f)}$ (cf. (2.25)) is $\mathbb{F}^{\mathrm{M}}$-adapted.

We define the vector $\left(L^{2}, L^{3}\right):=\left(\mathrm{M}\left(1_{[0, \cdot] \times\{|x|>1\}} x\right), \overline{\mathrm{M}}\left(1_{[0, \cdot] \times\{|x| \leq 1\}} x\right)\right)$ and the process $Y:=\left(Y_{t}\right)_{t \geq 0}$ by $Y_{t}:=L_{t}^{2}+L_{t}^{3}=L_{t}-\beta t-\mathrm{W}_{t}^{\sigma}, t \geq 0$. We denote by $\mathbb{F}^{Y}$ the completion of the filtration generated by $Y$. It is easy to see that $\mathbb{F}^{\mathrm{M}}$ coincides with $\mathbb{F}^{Y}$. Indeed, because of Proposition 2.3 .10 , the process $Y$ is clearly $\mathbb{F}^{\mathrm{M}}$-adapted 
and so $\mathbb{F}^{Y} \subseteq \mathbb{F}^{\mathrm{M}}$. Conversely, by the definition of $\mathrm{M}$, for $A \in \mathscr{B}(E)$ such that $A \subseteq[0, t] \times \mathbb{R}$, we have

$$
\mathrm{M}(A)=\sum_{0 \leq s \leq t} 1_{\left\{\Delta L_{s} \neq 0\right\}} 1_{A}\left(s, \Delta L_{s}\right)=\sum_{0 \leq s \leq t} 1_{\left\{\Delta Y_{s} \neq 0\right\}} 1_{A}\left(s, \Delta Y_{s}\right)
$$

which is $\mathscr{F}_{t}^{Y}$ measurable. Hence $\mathscr{F}_{t}^{Y} \supseteq \mathscr{F}_{t}^{\mathrm{M}}, t \geq 0$, i.e., $\mathbb{F}^{\mathrm{M}}=\mathbb{F}^{Y}$.

We observe that by Theorem 2.1.8, the process $Y$ is a Lévy process because $\left[L^{2}, L^{3}\right]=0$ and so the vector $\left(L^{2}, L^{3}\right)$ has $\mathbb{F}^{L}$ independent increments, hence independent increments. Therefore $\left(Y, \mathbb{F}^{Y}\right)$ is a Lévy process and $\mathbb{F}^{Y}$ satisfies the usual conditions. As a consequence, the filtration $\mathbb{F}^{\mathrm{M}}$ satisfies the usual conditions and we call it the natural filtration of M.

2.3.11 Proposition. Let $\left(L, \mathbb{F}^{L}\right)$ be a Lévy process with Gaussian part $\mathrm{W}^{\sigma}$ and jump measure M. The following identity holds:

$$
\mathbb{F}^{L}=\mathbb{F}^{\mathrm{W}^{\sigma}} \vee \mathbb{F}^{\mathrm{M}} .
$$

Proof. The process $\mathrm{W}^{\sigma}$ is a Wiener process relative to $\mathbb{F}^{L}$ (cf. Theorem 2.3.5). Moreover, from the definition of $Y$ we have that $Y$ is $\mathbb{F}^{L}$-adapted and so $\mathbb{F}^{Y} \subseteq \mathbb{F}^{L}$. On the other side, we saw that $\mathbb{F}^{\mathrm{M}}=\mathbb{F}^{Y}$ and hence $\mathbb{F}^{\mathrm{W}^{\sigma}} \vee \mathbb{F}^{\mathrm{M}} \subseteq \mathbb{F}^{L}$. From the Ito-Lévy decomposition of $L$ it is immediate to deduce that $L$ is $\mathbb{F}^{\mathrm{W}^{\bar{\sigma}}} \vee \mathbb{F}^{Y}$-adapted. Because of $\mathbb{F}^{\mathrm{M}}=\mathbb{F}^{Y}$, we can conclude that $\mathbb{F}^{\mathrm{W}^{\sigma}} \vee \mathbb{F}^{\mathrm{M}} \supseteq \mathbb{F}^{L}$ and the proof is finished. 



\section{CHAPTER 3}

\section{Martingale Problems for Lévy Processes}

Let $(\Omega, \mathscr{F}, \mathbb{P})$ be a probability space endowed with a Lévy process $L$. We denote by $\tilde{\mathscr{F}}_{\infty}^{L}$ the $\sigma$-algebra generated by $L$, i.e., $\tilde{\mathscr{F}}_{\infty}^{L}:=\sigma\left(L_{t}, t \geq 0\right)$. By $\mathscr{N}(\mathbb{P})$ we designate the $\mathbb{P}$-null sets of the $\sigma$-algebra $\tilde{\mathscr{F}}_{\infty}^{L}$ and by $\mathscr{F}_{\infty}^{L}$ we denote the $\mathbb{P}$-completion of $\tilde{\mathscr{F}}_{\infty}^{L}$ in itself, thus $\mathscr{F}_{\infty}^{L}:=\tilde{\mathscr{F}}_{\infty}^{L} \vee \mathscr{N}(\mathbb{P})$. We restrict our attention to the probability space $\left(\Omega, \mathscr{F}_{\infty}^{L}, \mathbb{P}\right)$. Obviously, $L$ is a Lévy process on this probability space. With the Lévy process $L$ we associate the filtration $\tilde{\mathbb{F}}^{L}=\left(\tilde{\mathscr{F}}_{t}^{L}\right)_{t \geq 0}$ by setting $\tilde{\mathscr{F}}_{t}^{L}:=\sigma\left(L_{s}, s \leq t\right)$ and we call it the filtration generated by $L$. By $\tilde{\mathbb{F}}_{+}^{L}=\left(\tilde{\mathscr{F}}_{t+}^{L}\right)_{t>0}$ we denote the smallest right-continuous filtration containing $\tilde{\mathbb{F}}^{L}$, i.e., such that $\tilde{\mathscr{F}}_{t}^{L} \subseteq \tilde{\mathscr{F}}_{t+}^{L}, t \geq 0$. The filtration $\mathbb{F}^{L}=\left(\mathscr{F}_{t}^{L}\right)_{t \geq 0}$ denotes the completion of $\tilde{\mathbb{F}}^{L}$ in $\mathscr{F}_{\infty}^{L}$, i.e., $\mathscr{F}_{t}^{L}:=$ $\tilde{\mathscr{F}}_{t}^{L} \vee \mathscr{N}(\mathbb{P}), t \geq 0$, while the filtration $\mathbb{F}_{+}^{L}=\left(\mathscr{F}_{t+}^{L}\right)_{t \geq 0}$ denotes the completion of $\tilde{\mathbb{F}}_{+}^{L}$ in $\mathscr{F}_{\infty}^{L}$, i.e., $\mathscr{F}_{t+}^{L}:=\tilde{\mathscr{F}}_{t+}^{L} \vee \mathscr{N}(\mathbb{P}), t \geq 0$. The filtration $\mathbb{F}_{+}^{L}$ satisfies the usual conditions and we call it the natural filtration of $L$. From Proposition 2.1.4, we know that also $\mathbb{F}^{L}$ satisfies the usual conditions and therefore we have $\mathbb{F}^{L}=\mathbb{F}_{+}^{L}$. However, the filtration $\mathbb{F}_{+}^{L}$ satisfies the usual conditions independently of the fact that $L$ is a $\mathbb{P}$-Lévy process, while to show that $\mathbb{F}^{L}$ is right continuous we need to know that $L$ is stochastically continuous and has independent increments (cf. Wang (1981)). The Lévy process $L$ is clearly a Lévy process relative to the filtration $\mathbb{F}_{+}^{L}$. The reference filtration in this chapter is $\mathbb{F}_{+}^{L}$. By $\mathrm{M}$ we denote the jump measure of the Lévy process $\left(L, \mathbb{F}_{+}^{L}\right)$. From $\S 2.3 .1$, we know that $\mathrm{M}$ is a Poisson random measure relative to $\mathbb{F}_{+}^{L}$. The intensity measure of $\mathrm{M}$ is $\mathrm{m}:=\lambda_{+} \otimes \nu$, where $\lambda_{+}$is the Lebesgue measure on $\left(\mathbb{R}_{+}, \mathscr{B}\left(\mathbb{R}_{+}\right)\right)$and $\nu$ is the Lévy measure of $L$ on $(\mathbb{R}, \mathscr{B}(\mathbb{R}))$. We denote by $\overline{\mathrm{M}}$ the compensated Poisson random measure of $L$, i.e., we put $\overline{\mathrm{M}}:=\mathrm{M}-\mathrm{m}$. In Theorem 2.2.16 we saw that for every function $f \in L^{2}(\nu)$ the process $X^{(f)}=\left(X_{t}^{(f)}\right)_{t \geq 0}$ defined by

$$
X_{t}^{(f)}:=\overline{\mathrm{M}}\left(1_{[0, t]} f\right), \quad t \geq 0,
$$

is a Lévy process relative to $\mathbb{F}_{+}^{L}$. Moreover, $X^{(f)}$ is an $\mathbb{F}_{+}^{L}$-martingale such that $X_{t}^{(f)} \in L^{2}(\mathbb{P}), t \geq 0$, that is, $X^{(f)}$ belongs to $\mathscr{H}_{\mathrm{loc}, 0}^{2}\left(\mathbb{F}_{+}^{L}\right)$. From Proposition 2.2.15, if $f$ is such that $|f| \wedge 1 \in L^{1}(\nu)$, then the process $\mathrm{M}\left(1_{\left[0,{ }^{\prime}\right]} f\right):=\left(\mathrm{M}\left(1_{[0, t]} f\right)\right)_{t \geq 0}$ is 
a Lévy process relative to $\mathbb{F}_{+}^{L}$. Furthermore, there exists a continuous process, say $\mathrm{W}^{\sigma}$, which is a Wiener process relative to the filtration $\mathbb{F}_{+}^{L}$ with variance function $\sigma^{2}(t)=\sigma^{2} t, \sigma^{2} \geq 0, t \geq 0$, such that

$$
L_{t}=\beta t+\mathrm{W}_{t}^{\sigma}+\mathrm{M}\left(1_{[0, t] \times\{|x|>1\} x}\right)+\overline{\mathrm{M}}\left(1_{[0, t] \times\{|x| \leq 1\} x}\right), \quad t \geq 0, \quad \text { a.s. },
$$

where $\beta \in \mathbb{R}$. This is the Itô-Lévy decomposition of $L$ (cf. Theorem 2.3.5). We call the process $\mathrm{W}^{\sigma}$ the Gaussian part of $L$. The family $\mathscr{X}$ defined by

$$
\mathscr{X}:=\left\{\mathrm{W}^{\sigma}\right\} \cup\left\{X^{(f)}, f \in L^{2}(\nu)\right\}
$$

is a family of locally square integrable $\mathbb{F}_{+}^{L}$-martingales starting at 0 and consequently $\mathscr{X} \subseteq \mathscr{H}_{\mathrm{loc}, 0}^{2}\left(\mathbb{F}_{+}^{L}\right)$. The aim of this chapter is to prove that the family $\mathscr{X}$ introduced in (3.1) possesses the $\mathscr{H}^{1}$-PRP with respect to $\mathbb{F}_{+}^{L}$, meaning that

$$
\mathscr{L}^{1}(\mathscr{X})=\mathscr{H}_{0}^{1}\left(\mathbb{F}_{+}^{L}\right) .
$$

We shall obtain (3.2) as a consequence of Theorem 1.4.12: This is the basic theoretical result of this chapter and we shall apply it to introduce other interesting families of martingales with the $\mathscr{H}^{1}$-PRP.

By $\mathscr{Q}(\mathscr{X})$ we denote the set of all the probability measures $\mathbb{Q}$ on $\left(\Omega, \tilde{\mathscr{F}}_{\infty}^{L}\right)$ solving the martingale problem associated with $\mathscr{X}$ :

$$
\mathscr{Q}(\mathscr{X}):=\left\{\mathbb{Q} \text { on }\left(\Omega, \tilde{\mathscr{F}}_{\infty}^{L}\right) \text { such that } \mathscr{X} \subseteq \mathscr{M}_{\mathrm{loc}, 0}\left(\mathbb{Q}, \mathbb{F}_{+}^{L}\right)\right\} .
$$

The set $\mathscr{Q}(\mathscr{X})$ is non-empty because the probability measure $\mathbb{P}$ belongs to it. Among the probability measures in $\mathscr{Q}(\mathscr{X})$, we consider the subset

$$
\mathscr{Q}_{e}(\mathscr{X}):=\left\{\mathbb{Q} \in \mathscr{Q}(\mathscr{X}): \mathbb{Q} \sim \mathbb{P} \text { on } \tilde{\mathscr{F}}_{\infty}^{L}\right\}
$$

of all the probability measures in $\mathscr{Q}(\mathscr{X})$ which are equivalent to $\mathbb{P}$ on $\left(\Omega, \tilde{\mathscr{F}}_{\infty}^{L}\right)$. We prove that $\mathbb{P}$ is in fact the unique element of $\mathscr{Q}_{e}(\mathscr{X})$. That is, we are going to verify the identity

$$
\mathscr{Q}_{e}(\mathscr{X})=\{\mathbb{P}\} .
$$

We observe that in a different context, a similar martingale problem was formulated in Jacod \& Shiryaev (2000) Theorem II.2.21. Notice that if $\mathbb{Q}$ is a probability measure on $\left(\Omega, \tilde{\mathscr{F}}_{\infty}^{L}\right)$ which is equivalent to $\mathbb{P}$, then the filtration $\mathbb{F}_{+}^{L}$ does not change because $\mathscr{N}(\mathbb{Q})=\mathscr{N}(\mathbb{P})$.

\subsection{The Gaussian Part}

Let $\mathbb{Q}$ be any probability measure on $\left(\Omega, \tilde{\mathscr{F}}_{\infty}^{L}\right)$ belonging to $\mathscr{Q}_{e}(\mathscr{X})$. We are going to verify that the Gaussian part of $L$, which is a $\mathbb{P}$-Wiener process relative to $\mathbb{F}_{+}^{L}$ with variance function $\sigma^{2}(t):=\sigma^{2} t, \sigma^{2} \geq 0$, remains a $\mathbb{Q}$-Wiener process relative to $\mathbb{F}_{+}^{L}$ with the same variance function. The Wiener process was introduced in Definition 1.2.8 and we stress that it is assumed to be a continuous process.

3.1.1 Proposition. The process $\left(\mathrm{W}^{\sigma}, \mathbb{F}_{+}^{L}\right)$ is a $\mathbb{Q}$-Wiener process with variance function $\sigma^{2}(t)=\sigma^{2} t$, for every $\mathbb{Q} \in \mathscr{Q}_{e}(\mathscr{X})$. 
Proof. The idea of the proof is to apply the theorem of P. Lévy on the characterization of the Wiener process (cf. Theorem 1.2.9). The process $\mathrm{W}^{\sigma}$ is a continuous $\mathbb{P}$-local martingale, because it is a Wiener process relative to $\mathbb{F}_{+}^{L}$ with respect to $\mathbb{P}$ (cf. Theorem 2.3.5). On the other side, $\mathrm{W}^{\sigma}$ is also a continuous local martingale with respect to $\mathbb{Q}$, because $\mathbb{Q}$ is a solution of the martingale problem associated with $\mathscr{X}$. Hence the process $\mathrm{W}^{\sigma} \cdot \mathrm{W}^{\sigma}$ is a local martingale both with respect to $\mathbb{P}$ and $\mathbb{Q}$. By Ito's formula we have

$$
2 \mathrm{~W}^{\sigma} \cdot \mathrm{W}_{t}^{\sigma}=\left(\mathrm{W}_{t}^{\sigma}\right)^{2}-\sigma^{2} t, \quad t \geq 0, \quad \mathbb{P} \text {-a.s. }
$$

and, because of the equivalence of the probability measures, the previous equality holds also $\mathbb{Q}$-a.s. So, we can conclude that the process $\left(\left(\mathrm{W}_{t}^{\sigma}\right)^{2}-\sigma^{2} t, \mathscr{F}_{t+}^{L}, t \geq 0\right)$ is a $\mathbb{Q}$-local martingale. Therefore

$$
\left\langle\mathrm{W}^{\sigma}, \mathrm{W}^{\sigma}\right\rangle_{t}=\sigma^{2} t, \quad t \geq 0, \quad \mathbb{P} \text {-a.s. and } \mathbb{Q} \text {-a.s. }
$$

By Theorem 1.2.9, $\mathrm{W}^{\sigma}$ is a $\mathbb{Q}$-Wiener process relative to the filtration $\mathbb{F}_{+}^{L}$ with variance function $\sigma^{2}(t)=\sigma^{2} t$.

\subsection{The Jump Measure}

The process $\left(L, \mathbb{F}_{+}^{L}\right)$ is a Lévy process with respect to the probability $\mathbb{P}$. Hence, by definition, $L$ is also an $\mathbb{F}_{+}^{L}$-adapted càdlàg process and these properties do not depend on the probability measure with respect to which $L$ is considered. Therefore the random measure $\mathrm{M}$ of the jumps of $L$, which with respect to $\mathbb{P}$ is a Poisson random measure relative to $\mathbb{F}_{+}^{L}$ with intensity measure $\lambda_{+} \otimes \nu$, remains an integer-valued random measure (not necessarily a Poisson random measure) also with respect to any other probability measure $\mathbb{Q}$ on $\left(\Omega, \tilde{\mathscr{F}}_{\infty}^{L}\right)$ (cf. Proposition 2.2.1). Our aim is to show that with respect to any $\mathbb{Q}$ in $\mathscr{Q}_{e}(\mathscr{X})$, the integer-valued random measure $\mathrm{M}$ remains in fact a Poisson random measure relative to $\mathbb{F}_{+}^{L}$ with intensity measure $\lambda_{+} \otimes \nu$. Let $\nu$ be the Lévy measure of $L$ with respect to $\mathbb{P}$ and let $A \in \mathscr{B}(E)$ be of the form $A=(r, v] \times C$, where $0 \leq r<v$ and $C \in \mathscr{B}(\mathbb{R})$ is such that $\nu(C)<+\infty$. For such an $A$, we define the process $N^{A}=\left(N_{t}^{A}\right)_{t \geq 0}$ by

$$
N_{t}^{A}:=\mathrm{M}(A \cap[0, t] \times \mathbb{R}), \quad t \geq 0, \quad A=(r, v] \times C, \quad \nu(C)<+\infty .
$$

Because of Lemma 2.2.4, the process $\left(N^{A}, \mathbb{F}_{+}^{L}\right)$ is a $\mathbb{P}$-Poisson process with intensity function $a(\cdot):=\left(\lambda_{+} \otimes \nu\right)(A \cap[0, \cdot] \times \mathbb{R})$. As first step, we show that with respect to each probability measure $\mathbb{Q} \in \mathscr{Q}_{e}(\mathscr{X})$ the process $N^{A}$ remains a Poisson process relative to $\mathbb{F}_{+}^{L}$ with the same intensity function. We stress that now the situation is different from the one that we met in Chapter 2. Indeed, in $\S 2.3 .1$ we considered a filtration $\mathbb{F}$ satisfying the usual conditions and required that $\mathrm{M}$ was the random measure associated with the jumps of the $\mathbb{P}$-Lévy process $(L, \mathbb{F})$. Under these assumptions, we proved that $\mathrm{M}$ is a Poisson random measure relative to the filtration $\mathbb{F}$ with intensity $\lambda_{+} \otimes \nu$ with respect to $\mathbb{P}$. Now we do not know that $\left(L, \mathbb{F}_{+}^{L}\right)$ is a $\mathbb{Q}$-Lévy process and we cannot proceed as in $\S 2.3 .1$ to prove that $\left(N^{A}, \mathbb{F}_{+}^{L}\right)$ is a 
$\mathbb{Q}$-Poisson process. Instead, we shall prove this result with the help of the martingale problem and as a consequence we shall deduce that $\mathrm{M}$ is a $\mathbb{Q}$-Poisson random measure relative to $\mathbb{F}_{+}^{L}$. We recall that we use the following notation

$$
(E, \mathscr{B}(E)):=\left(\mathbb{R}_{+} \times \mathbb{R}, \mathscr{B}\left(\mathbb{R}_{+}\right) \otimes \mathscr{B}(\mathbb{R})\right) .
$$

3.2.1 Proposition. Let $\nu$ be the Lévy measure with respect to $\mathbb{P}$ of the $\mathbb{P}$-Lévy process $\left(L, \mathbb{F}_{+}^{L}\right)$ and let $\mathbb{Q} \in \mathscr{Q}_{e}(\mathscr{X})$. Then the following assertions hold.

(i) Let $A \in \mathscr{B}(E)$ be of the form $A=(r, v] \times C$, where $0 \leq r<v$ and $C \in \mathscr{B}(\mathbb{R})$ is such that $\nu(C)<+\infty$. For every such an $A$, the process $N^{A}$ defined by (3.5) is a $\mathbb{Q}$-Poisson process relative to the filtration $\mathbb{F}_{+}^{L}$ and its intensity function is $a^{A}(\cdot):=\left(\lambda_{+} \otimes \nu\right)(A \cap[0, \cdot] \times \mathbb{R})$.

(ii) We fix $s>0$. Let $A_{1}, \ldots, A_{m}, m \in \mathbb{N}$, be pairwise disjoint Borel subsets of $(s,+\infty) \times \mathbb{R}$ of the form $A_{j}=\left(r_{j}, v_{j}\right] \times C_{j}$, where $s<r_{j}<v_{j}$ and $C_{j} \in \mathscr{B}(\mathbb{R})$ such that $\nu\left(B_{j}\right)<+\infty, j=1, \ldots, m$. Then the vector $\left(\mathrm{M}\left(A_{1}\right), \ldots, \mathrm{M}\left(A_{m}\right)\right)$ is $\mathbb{Q}$-independent of $\mathscr{F}_{s+}^{L}, m \in \mathbb{N}$.

Proof. (i) The set $A$ is of the form $A=(r, v] \times C$ where $0 \leq r<v$ and $C \in \mathscr{B}(\mathbb{R})$ is such that $\nu(C)<+\infty$. The process $N^{A}$ is a Poisson process under $\mathbb{P}$ and, by the equivalence of the measures, it remains a simple point process with respect to $\mathbb{Q} \in \mathscr{Q}_{e}(\mathscr{X})$. Indeed, because $\nu(C)<+\infty$ we have $\mathrm{M}(A \cap[0, t] \times \mathbb{R})<+\infty \mathbb{P}$-a.s., and so $\mathbb{Q}$-a.s., $t \geq 0$. For the other properties characterizing a simple point process cf. Lemma 2.2.2 and the comment following it. Moreover, for every $t \geq 0$, we have

$$
\begin{aligned}
& N_{t}^{A}-\left(\lambda_{+} \otimes \nu\right)(A \cap[0, t] \times \mathbb{R}) \\
& =\left(\mathrm{M}([0, t] \times C)-\left(\lambda_{+} \otimes \nu\right)([0, t] \times C)\right)^{v}-\left(\mathrm{M}([0, t] \times C)-\left(\lambda_{+} \otimes \nu\right)([0, t] \times C)\right),{ }^{r}
\end{aligned}
$$

where the superscripts $v$ and $r$ denote the stopping operation at the deterministic time $v$ and $r$, respectively. Because of $\nu(C)<+\infty$, the function $f:=1_{C}$ belongs to $L^{1}(\nu) \cap L^{2}(\nu)$. Hence, from Proposition 2.2.12 and from the equivalence of $\mathbb{P}$ and $\mathbb{Q}$, it follows

$$
\mathrm{M}\left(1_{[0, t]} f\right)-t \nu(C)=X_{t}^{(f)}, \quad t \geq 0, \quad \mathbb{P} \text {-a.s. } \quad \text { and } \quad \mathbb{Q} \text {-a.s. }
$$

Consequently, $\left(\mathrm{M}\left(1_{[0, t]} f\right)-t \nu(C), \mathscr{F}_{t+}^{L}, t \geq 0\right) \in \mathscr{M}_{\mathrm{loc}, 0}(\mathbb{Q})$ because $X^{(f)} \in \mathscr{X}$ and $\mathbb{Q}$ solves the martingale problem associated with $\mathscr{X}$. The space $\mathscr{M}_{\mathrm{loc}, 0}(\mathbb{Q})$ is linear and stable under stopping, therefore $\left(N_{t}^{A}-\left(\lambda_{+} \otimes \nu\right)(A \cap[0, t] \times \mathbb{R}), \mathscr{F}_{t+}^{L}, t \geq 0\right)$ is a $\mathbb{Q}$-local martingale as a difference of two local martingales. By Theorem 1.2.7 we can conclude that $\left(N^{A}, \mathbb{F}_{+}^{L}\right)$ is a Poisson process with intensity function $a^{A}(\cdot):=$ $\left(\lambda_{+} \otimes \nu\right)(A \cap[0, \cdot] \times \mathbb{R})$ with respect to each $\mathbb{Q} \in \mathscr{Q}_{e}(\mathscr{X})$.

(ii) We fix $s>0$ and consider $A_{1}, \ldots, A_{m}, m \in \mathbb{N}$, pairwise disjoint Borel subsets of $(s,+\infty) \times \mathbb{R}$ of the form $A_{j}=\left(r_{j}, v_{j}\right] \times C_{j}$, where $s<r_{j}<v_{j}$ and $C_{j} \in \mathscr{B}(\mathbb{R})$ is such that $\nu\left(C_{j}\right)<+\infty, j=1, \ldots, m$. Because of (i), $N^{A_{j}}$ is a semimartingale and an additive process relative to $\mathbb{F}_{+}^{L}$ with respect to $\mathbb{Q}, j=1, \ldots, m$. For $j \neq k$, $j, k=1, \ldots, m$, we have $A_{j} \cap A_{k}=\emptyset$ and this yields

$$
\begin{aligned}
{\left[N^{A_{j}}, N^{A_{k}}\right]_{t} } & =\sum_{0<s \leq t} \Delta N_{s}^{A_{j}} \Delta N_{s}^{A_{k}} \\
& =\sum_{0<s \leq t} 1_{\left\{\Delta L_{s} \neq 0\right\}} 1_{A_{j} \cap A_{k}}\left(s, \Delta L_{s}\right)=0, \quad t \geq 0, \quad \mathbb{P} \text {-a.s. and } \mathbb{Q} \text {-a.s. }
\end{aligned}
$$


Hence we can apply Theorem 2.1.8 to obtain that the vector $\left(N^{A_{1}}, \ldots, N^{A_{m}}\right)$ has $\mathbb{F}_{+}^{L}$-independent increments with respect to $\mathbb{Q}$. On the other hand, $A_{j} \subseteq(s,+\infty) \times \mathbb{R}$ implies that $N_{s}^{A_{j}}=0, j=1, \ldots, m$. Therefore, the vector $\left(N_{t}^{A_{1}}, \ldots, N_{t}^{A_{m}}\right)$ is $\mathbb{Q}$ independent of $\mathscr{F}_{s+}^{L}$ because $\left(N_{t}^{A_{1}}, \ldots, N_{t}^{A_{m}}\right)=\left(N_{t}^{A_{1}}-N_{s}^{A_{1}}, \ldots, N_{t}^{A_{m}}-N_{s}^{A_{m}}\right)$, $t \geq 0$. But $\left(N_{t}^{A_{1}}, \ldots, N_{t}^{A_{m}}\right)$ converges pointwise in $\omega$ to $\left(\mathrm{M}\left(A_{1}\right), \ldots, \mathrm{M}\left(A_{m}\right)\right)$ as $t \rightarrow+\infty$ and so we can assert that the latter vector is $\mathbb{Q}$-independent of $\mathscr{F}_{s+}^{L}$, $m \in \mathbb{N}$, and the proof of the proposition is finished.

Now we show that the intensity measure of the integer-valued random measure $\mathrm{M}$ is again $\lambda_{+} \otimes \nu$, with respect to any $\mathbb{Q} \in \mathscr{Q}_{e}(\mathscr{X})$.

3.2.2 Proposition. Let $\mathrm{M}$ be the jump measure of $L$. With respect to any $\mathbb{Q} \in$ $\mathscr{Q}_{e}(\mathscr{X})$, the intensity measure of $\mathrm{M}$ is $\lambda_{+} \otimes \nu$.

Proof. We have to prove the relation

$$
\mathbb{E}_{\mathbb{Q}}[\mathrm{M}(A)]=\left(\lambda_{+} \otimes \nu\right)(A), \quad A \in \mathscr{B}(E) .
$$

Let $\mathrm{m}$ be the intensity of $\mathrm{M}$ with respect to $\mathbb{Q} \in \mathscr{Q}_{e}(\mathscr{X})$, i.e.,

$$
\mathrm{m}(A)=\mathbb{E}_{\mathbb{Q}}[\mathrm{M}(A)], \quad A \in \mathscr{B}(E) .
$$

We are going to show $\mathrm{m}=\lambda_{+} \otimes \nu$ on $\mathscr{B}(E)$. We introduce the system of sets

$$
\begin{aligned}
\mathscr{C}:=\{\{0\} \times C, & C \in \mathscr{B}(\mathbb{R}): \nu(C)<+\infty\} \cup \\
& \cup\{(r, v] \times C: 0 \leq r \leq v, C \in \mathscr{B}(\mathbb{R}): \nu(C)<+\infty\}
\end{aligned}
$$

which generates $\mathscr{B}(E)$ and is stable under intersections. Proposition 3.2.1 implies that for $A \in \mathscr{C}$ the process $N^{A}$ is a $\mathbb{Q}$-Poisson process relative to $\mathbb{F}_{+}^{L}$ and its intensity function is given by $a^{A}(\cdot):=\left(\lambda_{+} \otimes \nu\right)(A \cap[0, t] \times \mathbb{R})$. We have

$$
\mathrm{M}(A)=\mathrm{M}((r, v] \times C)=N_{t}^{A}, \quad t \geq v
$$

and, for $v=t$, we get

$$
\mathrm{m}(A)=\mathbb{E}_{\mathbb{Q}}[\mathrm{M}(A)]=\mathbb{E}_{\mathbb{Q}}[\mathrm{M}((r, v] \times C)]=\mathbb{E}_{\mathbb{Q}}\left[N_{v}^{A}\right]=\left(\lambda_{+} \otimes \nu\right)(A) .
$$

Furthermore, the sets $A_{n}:=[0, n] \times\left(\left\{|x|>\frac{1}{n}\right\} \cup\{0\}\right), n \geq 1$, belong to $\mathscr{C}$ and are such that $\cup_{n=1}^{\infty} A_{n}=E$. Therefore, $\left(A_{n}\right)_{n \geq 1}$ is a sequence of sets of finite measure both with respect to $\mathrm{m}$ and $\lambda_{+} \otimes \nu$. An application of the uniqueness theorem for measures (cf. Theorem 1.1.2) shows that $\mathrm{m}=\lambda_{+} \otimes \nu$ on $\mathscr{B}(E)$.

To conclude that $\mathrm{M}$ is a Poisson random measure relative to the filtration $\mathbb{F}_{+}^{L}$ with respect to each $\mathbb{Q} \in \mathscr{Q}_{e}(\mathscr{X})$, we need to verify the independence property of Poisson random measures relative to the filtration.

3.2.3 Proposition. Let $\mathbb{Q}$ be any probability measure in $\mathscr{Q}_{e}(\mathscr{X})$ and let $\mathrm{M}$ be the the jump measure of $L$. For every fixed $s \in \mathbb{R}_{+}$and every $A \in \mathscr{B}(E)$ such that $A \subseteq$ $(s,+\infty) \times \mathbb{R}$ and $\left(\lambda_{+} \otimes \nu\right)(A)<+\infty$, the random variable $\mathrm{M}(A)$ is $\mathbb{Q}$-independent of $\mathscr{F}_{s+}^{L}$. 
Proof. Let $A_{1}, \ldots, A_{m} \in \mathscr{B}(E)$ be pairwise disjoint sets contained in $(s,+\infty) \times \mathbb{R}$ of the form $A_{j}=\left(r_{j}, v_{j}\right] \times C_{j}$, where $s<r_{j} \leq v_{j}$ and $C_{j} \in \mathscr{B}(\mathbb{R})$ is such that $\nu\left(C_{j}\right)<+\infty$, for every $j=1, \ldots, m$. We recall that because of Proposition 3.2.2, we know that the intensity measure of $\mathrm{M}$ is $\lambda_{+} \otimes \nu$. By Proposition 3.2.1, the random vector $\left(\mathrm{M}\left(A_{1}\right), \ldots, \mathrm{M}\left(A_{m}\right)\right)$ is $\mathbb{Q}$-independent of $\mathscr{F}_{s+}^{L}$. Now we consider the case $A_{j} \subseteq(s, T) \times\{|x|>\varepsilon\}$, for every $j=1, \ldots, m$, where $T>s$ and $\varepsilon>0$ are arbitrary but fixed.

$$
\begin{aligned}
\mathscr{C}:=\left\{\bigcup_{j=1}^{n} A_{j}, A_{j}:=\left(r_{j}, v_{j}\right] \times B_{j} \text { p.d., } \quad s<r_{j} \leq v_{j} \leq T,\right. \\
\left.B_{j} \in \mathscr{B}(\mathbb{R}): B_{j} \subseteq\{|x|>\varepsilon\}\right\},
\end{aligned}
$$

where the acronym $p . d$. stands for pairwise disjoint. We notice that $\mathscr{C}$ is an algebra of measurable subsets of $(s, T) \times\{|x|>\varepsilon\}$ (cf. Definition 1.1.1) and it generates the $\sigma$-fields $\mathscr{B}((s, T)) \otimes \mathscr{B}(\{|x|>\varepsilon\})$. For any $A \in \mathscr{C}$ it follows

$$
\mathrm{M}(A)=\mathrm{M}\left(\bigcup_{j=1}^{n}\left(r_{j}, v_{j}\right] \times B_{j}\right)=\sum_{j=1}^{n} \mathrm{M}\left(\left(r_{j}, v_{j}\right] \times B_{j}\right) .
$$

Because of the previous step the vector $\left(\mathrm{M}\left(\left(r_{1}, v_{1}\right] \times B_{1}\right), \ldots, \mathrm{M}\left(\left(r_{n}, v_{n}\right] \times B_{n}\right)\right)$ is $\mathbb{Q}$-independent of $\mathscr{F}_{s+}^{L}$, which implies that $\mathrm{M}(A)$ is $\mathbb{Q}$-independent of $\mathscr{F}_{s+}^{L}$. The class $\mathscr{A} \subseteq \mathscr{B}(E)$ defined by

$$
\mathscr{A}:=\left\{A \subseteq(s, T) \otimes\{|x|>\varepsilon\} \text { Borel subset : } \mathrm{M}(A) \text { is } \mathbb{Q} \text {-independent of } \mathscr{F}_{s+}^{L}\right\}
$$

is a monotone class of sets (cf. $\S 1.1 .2)$. Indeed, if $\left(A_{n}\right)_{n \geq 1} \subseteq \mathscr{A}$ is such that $A_{n} \uparrow A$ or that $A_{n} \downarrow A$, then $A \in \mathscr{A}$. By the previous step, $\mathscr{C}$ is contained in $\mathscr{A}$ and an application of the monotone class theorem for systems of sets (cf. Theorem 1.1.5) yields $\mathscr{A}=\mathscr{B}((s, T)) \otimes \mathscr{B}(\{|x|>\varepsilon\})$. Let now $A$ be an arbitrary Borel subset of $(s,+\infty) \times \mathbb{R}$ such that $\left(\lambda_{+} \otimes \nu\right)(A)<+\infty$. We can define the sequence $\left(A_{n}\right)_{n \geq 1}$ by

$$
A_{n}:=A \cap(s, n] \times\left\{|x|>\frac{1}{n}\right\}, \quad n \geq 1 .
$$

Then $A_{n} \uparrow A \backslash(s,+\infty) \times\{0\}$. Hence $\mathrm{M}\left(A_{n}\right) \uparrow \mathrm{M}(A \backslash(s,+\infty) \times\{0\})$ and the convergence is pointwise. On the other hand, $\mathrm{M}(A \backslash(s,+\infty) \times\{0\})=\mathrm{M}(A), \mathbb{Q}$ a.s. because $\lambda_{+} \otimes \nu$ is the intensity measure of $\mathrm{M}$ with respect to $\mathbb{Q}$ and clearly $\left(\lambda_{+} \otimes \nu\right)(A \backslash(s,+\infty) \times\{0\})=\left(\lambda_{+} \otimes \nu\right)(A)$. From the previous step, $\mathrm{M}\left(A_{n}\right)$ is $\mathbb{Q}$-independent of $\mathscr{F}_{s+}^{L}$ for every $n \geq 1$. Therefore the same holds for $\mathrm{M}(A)$ and the proof is complete.

The jump measure $\mathrm{M}$ of the process $L$ is an integer-valued random measure because $L$ is an $\mathbb{F}_{+}^{L}$-adapted càdlàg process. From Proposition 3.2.2 and Proposition 3.2.3 it follows that $\mathrm{M}$ is a Poisson random measure relative to the filtration $\mathbb{F}_{+}^{L}$ with intensity measure $\lambda_{+} \otimes \nu$ also with respect to any probability measure $\mathbb{Q}$ in $\mathscr{Q}_{e}(\mathscr{X})$. In other words, we have proven the following theorem.

3.2.4 Theorem. The jump measure $\mathrm{M}$ of the process $L$ is a Poisson random measure relative to the filtration $\mathbb{F}_{+}^{L}$ with intensity measure $\mathrm{m}=\lambda_{+} \otimes \nu$ with respect to each $\mathbb{Q} \in \mathscr{Q}_{e}(\mathscr{X})$. 


\subsection{Uniqueness of the Solution of the Martingale Problem}

Let $\left(L, \mathbb{F}_{+}^{L}\right)$ be a $\mathbb{P}$-Lévy process with characteristic triplet $\left(\beta, \sigma^{2}, \nu\right)$. The Itô-Lévy decomposition of $L$ with respect to $\mathbb{P}$ is

$$
L_{t}=\beta t+\mathrm{W}_{t}^{\sigma}+\mathrm{M}\left(1_{[0, t] \times\{|x|>1\}} x\right)+\overline{\mathrm{M}}\left(1_{[0, t] \times\{|x| \leq 1\}} x\right), \quad t \geq 0, \quad \mathbb{P} \text {-a.s. }
$$

In Proposition 3.1.1 we showed that under any probability measure $\mathbb{Q}$ equivalent to $\mathbb{P}$ on $\left(\Omega, \tilde{\mathscr{F}}_{\infty}^{L}\right)$ which is a solution of the martingale problem associated with $\mathscr{X}$, the process $\left(\mathrm{W}^{\sigma}, \mathbb{F}_{+}^{L}\right)$ remains a Wiener process with variance function $\sigma^{2}(t)=\sigma^{2} t$. In Theorem 3.2.4 we saw that, if $\mathbb{Q} \in \mathscr{Q}_{e}(\mathscr{X})$, then the jump measure $\mathrm{M}$ of $L$ remains a Poisson random measure relative to the filtration $\mathbb{F}_{+}^{L}$ with intensity measure $\mathrm{m}=$ $\lambda_{+} \otimes \nu$ with respect to $\mathbb{Q}$. In this section we obtain that the set $\mathscr{Q}_{e}(\mathscr{X})$ defined in (3.4) is equal to the singleton $\{\mathbb{P}\}$.

3.3.1 Theorem. Let $\left(L, \mathbb{F}_{+}^{L}\right)$ be a Lévy process on the probability space $\left(\Omega, \mathscr{F}_{\infty}^{L}, \mathbb{P}\right)$ with characteristic triplet $\left(\beta, \sigma^{2}, \nu\right)$ and Itô-Lévy decomposition

$$
L_{t}=\beta t+\mathrm{W}_{t}^{\sigma}+\mathrm{M}\left(1_{[0, t] \times\{|x|>1\}} x\right)+\overline{\mathrm{M}}\left(1_{[0, t] \times\{|x| \leq 1\}} x\right), \quad t \geq 0, \quad \mathbb{P} \text {-a.s. }
$$

where $\mathrm{W}^{\sigma}$ and $\mathrm{M}$ are the Gaussian part and the jump measure of L, respectively. Then

$$
\mathscr{Q}_{e}(\mathscr{X})=\{\mathbb{P}\} .
$$

Proof. Let $\mathbb{Q} \in \mathscr{Q}_{e}(\mathscr{X})$. Because of the equivalence of $\mathbb{P}$ and $\mathbb{Q}$ we can write

$$
L_{t}=\beta t+\mathrm{W}_{t}^{\sigma}+\mathrm{M}\left(1_{[0, t] \times\{|x|>1\}} x\right)+\overline{\mathrm{M}}\left(1_{[0, t] \times\{|x| \leq 1\}} x\right), \quad t \geq 0, \quad \mathbb{Q} \text {-a.s. },
$$

where $\mathrm{M}$ is the jump meausre of $L$. By Theorem 3.2.4, we know that $\mathrm{M}$ is a Poisson random measure relative to the filtration $\mathbb{F}_{+}^{L}$ with intensity measure $\lambda_{+} \otimes \nu$ also with respect to $\mathbb{Q}$. By Proposition 3.1.1, $\left(\mathrm{W}^{\sigma}, \mathbb{F}_{+}^{L}\right)$ is a $\mathbb{Q}$-Wiener process whose variance function is $\left\langle\mathrm{W}^{\sigma}, \mathrm{W}^{\sigma}\right\rangle_{t}=\sigma^{2} t$. From (3.6) and Theorem 2.3.5, we can conclude that $\left(L, \mathbb{F}_{+}^{L}\right)$ is a Lévy process also with respect to $\mathbb{Q}$. In other words, (3.6) is the ItôLévy decomposition of $L$ with respect to $\mathbb{Q}$. The characteristic triplet of $\left(L, \mathbb{F}_{+}^{L}\right)$ with respect to $\mathbb{Q}$ is equal to $\left(\beta, \sigma^{2}, \nu\right)$, which was the characteristic triplet of $\left(L, \mathbb{F}_{+}^{L}\right)$ as a $\mathbb{P}$-Lévy process. Theorem 2.3.6 implies that $\mathbb{Q}$ is equal to $\mathbb{P}$ on $\tilde{\mathscr{F}}_{\infty}^{L}$ and so

$$
\mathscr{Q}_{e}(\mathscr{X})=\{\mathbb{P}\} .
$$

We recall that as a consequence of Theorem 2.1.8 the summands appearing in (3.6) are $\mathbb{Q}$-independent. Moreover, the Wiener process $\mathrm{W}^{\sigma}$ and the Poisson random measure $\mathrm{M}$ are $\mathbb{Q}$-independent (cf. the comment following Theorem 2.3.5). We conclude this section deducing the result on the $\mathscr{H}^{1}$-PRP as an immediate consequence of Theorem 3.3.1 and Theorem 1.4.12.

3.3.2 Theorem. Let $\left(L, \mathbb{F}_{+}^{L}\right)$ be a Lévy process with characteristic triplet $\left(\beta, \sigma^{2}, \nu\right)$. The family $\mathscr{X}$ defined in (3.1) has the $\mathscr{H}^{1}-P R P$ with respect to the filtration $\mathbb{F}_{+}^{L}$, i.e.,

$$
\mathscr{L}^{1}(\mathscr{X})=\mathscr{H}_{0}^{1}\left(\mathbb{F}_{+}^{L}\right)
$$


Proof. Thanks to Theorem 3.3.1 we get, as an application of Theorem 1.4.12, that

$$
\mathscr{L}^{1}(\mathscr{X})=\mathscr{H}_{0}^{1}\left(\mathbb{F}_{+}^{L}\right)
$$

\subsection{Representation of Martingales}

In the previous section, given a Lévy process $\left(L, \mathbb{F}_{+}^{L}\right)$ with characteristic triplet $\left(\beta, \sigma^{2}, \nu\right)$, we have proven that the family $\mathscr{X}$ defined in (3.1) posses the $\mathscr{H}^{1}$-PRP with respect to $\mathbb{F}_{+}^{L}$. Notice that the filtrations $\mathbb{F}_{+}^{L}$ and $\mathbb{F}^{L}$ coincide: From now on, we shall consider only the latter one. Using Theorem 3.3.2 as a starting point, we want to construct other interesting families of martingales possessing the $\mathscr{H}^{1}$-PRP. The main result of this section is that with every total system $\mathscr{T} \subseteq L^{2}(\nu)$ it is possible to associate a family of martingales with the $\mathscr{H}^{1}$-PRP. Before we establish a technical lemma.

3.4.1 Lemma. Let $M \in \mathscr{H}_{\mathrm{loc}, 0}^{q}$ and let $\left(T_{n}\right)_{n \geq 1}$ be a sequence of stopping times localizing $M$ to $\mathscr{H}_{0}^{q}$, i.e., $T_{n} \uparrow+\infty$ as $n \rightarrow+\infty$ and $M^{T_{n}} \in \mathscr{H}_{0}^{q}, n \geq 1$. Then

$$
\mathscr{L}^{q}\left(\left\{M^{T_{n}}, n \geq 1\right\}\right)=\mathscr{L}^{q}(M) .
$$

Proof. By Lemma 1.4.4, the inclusion $\left\{M^{T_{n}}: n \geq 1\right\} \subseteq \mathscr{L}^{q}(M)$ holds. Therefore, by the stability of $\mathscr{L}^{q}(M), \mathscr{L}^{q}\left(\left\{M^{T_{n}}, n \geq 1\right\}\right) \subseteq \mathscr{L}^{q}(M)$. For the converse inclusion it is enough to show that every element of the form $H \cdot M, H \in \mathrm{L}^{q}(M)$ (cf. (1.26)), belongs to $\mathscr{L}^{q}\left(\left\{M^{T_{n}}, n \geq 1\right\}\right.$ ) because $\mathscr{L}^{q}(M)=\left\{H \cdot M, M \in \mathrm{L}^{q}(M)\right\}$ (cf. Theorem 1.4.5.(i)). From Proposition 1.3.4.(ii), we have that the martingale $X:=H \cdot M$, $H \in \mathrm{L}^{q}(M)$, belongs to $\mathscr{H}_{0}^{q}$. Moreover,

$$
X^{T_{n}}=(H \cdot M)^{T_{n}}=\left(H 1_{\left[0, T_{n}\right]}\right) \cdot M=\left(H 1_{\left[0, T_{n}\right]}\right) \cdot M^{T_{n}}
$$

(cf. (1.31)). From the last of these equalities, we get $X^{T_{n}} \in \mathscr{L}^{q}\left(\left\{M^{T_{n}}, n \geq 1\right\}\right)$, $n \geq 1$. We define

$$
H^{n}:=H 1_{\left[0, T_{n}\right]}, \quad n \geq 1 .
$$

Clearly, $H^{n}$ converges pointwise to $H$ as $n \rightarrow+\infty$ and $\left|H^{n}\right| \leq|H| \in \mathrm{L}^{q}(M)$. By Proposition 1.3.5, we get $H^{n} \cdot M \longrightarrow H \cdot M$ in $\left(\mathscr{H}_{0}^{q},\|\cdot\|_{\mathscr{H}^{q}}\right)$ as $n \rightarrow+\infty$. But the space $\mathscr{L}^{q}\left(\left\{M^{T_{n}}: n \geq 1\right\}\right)$ is closed in $\left(\mathscr{H}_{0}^{q},\|\cdot\|_{\mathscr{H}^{q}}\right)$ and hence the integral process $H \cdot M$ belongs to it.

Let $\left(L, \mathbb{F}^{L}\right)$ be a Lévy process with characteristic triplet $\left(\beta, \sigma^{2}, \nu\right)$ and let $\mathscr{T}$ be a subset of $L^{2}(\nu)$. We define the family

$$
\mathscr{X}_{\mathscr{T}}:=\left\{\mathrm{W}^{\sigma}\right\} \cup\left\{X^{(f)}, \quad f \in \mathscr{T}\right\} .
$$

Now we prove that the totality in $L^{2}(\nu)$ of the system $\mathscr{T}$ is a sufficient condition for the associated family $\mathscr{X}_{\mathscr{T}}$ of martingales to have the $\mathscr{H}^{1}$-PRP with respect to $\mathbb{F}^{L}$. 
3.4.2 Theorem. Let $\left(L, \mathbb{F}^{L}\right)$ be a Lévy process with characteristic triplet $\left(\beta, \sigma^{2}, \nu\right)$. If $\mathscr{T}$ is total in $L^{2}(\nu)$, then the family $\mathscr{X}_{\mathscr{T}}$ has the $\mathscr{H}^{1}-P R P$ with respect to $\mathbb{F}^{L}$.

Proof. For $N \in \mathbb{N}$, we introduce the families

$$
\mathscr{X}^{N}:=\left\{\mathrm{W}^{\sigma N}\right\} \cup\left\{X^{(f)^{N}}, \quad f \in L^{2}(\nu)\right\}, \quad \mathscr{X}_{\mathscr{T}}^{N}:=\left\{\mathrm{W}^{\sigma N}\right\} \cup\left\{X^{(f)^{N}}, \quad f \in \mathscr{T}\right\},
$$

where the superscript $N$ denotes the operation of stopping at the deterministic time $N \in \mathbb{N}$. Under the assumption that $\mathscr{T}$ is total in $L^{2}(\nu)$, we know that for every $f \in L^{2}(\nu)$ there exists a sequence $\left(f_{n}\right)_{n \in \mathbb{N}} \subseteq \operatorname{Span}(\mathscr{T})$ such that $f_{n} \longrightarrow f$ in $L^{2}(\nu)$ as $n \rightarrow+\infty$. Because of the linearity of $X^{(g)}$ with respect to $g \in L^{2}(\nu)$, we can conclude that $X^{\left(f_{n}\right)} \in \operatorname{Span}\left(\mathscr{X}_{\mathscr{T}}\right)$. By isometry, for any $N \in \mathbb{N}$, we get

$$
\left\|X_{N}^{(f)}-X_{N}^{\left(f_{n}\right)}\right\|_{L^{2}(\mathbb{P})}=\left\|X_{N}^{\left(f-f_{n}\right)}\right\|_{L^{2}(\mathbb{P})}=\sqrt{N}\left\|f-f_{n}\right\|_{L^{2}(\nu)} \longrightarrow 0, \quad n \rightarrow+\infty .
$$

The processes $X^{(f)^{N}}$ and $X^{\left(f_{n}\right)^{N}}$ belong to $\mathscr{H}_{0}^{2}$ for every $N \in \mathbb{N}$ (cf. Theorem 2.2.16.(iii)) and by Doob's inequality we get that $X^{\left(f_{n}\right)^{N}} \longrightarrow X^{(f)^{N}}$ in $\left(\mathscr{H}_{0}^{2},\|\cdot\|_{\mathscr{H}^{2}}\right)$ as $n \rightarrow+\infty$. Hence the convergence holds in $\left(\mathscr{H}_{0}^{1},\|\cdot\|_{\mathscr{H}^{1}}\right)$, for every $N \in \mathbb{N}$. This implies that

$$
\mathscr{X}^{N} \subseteq \overline{\operatorname{Span}\left(\mathscr{X}_{\mathscr{T}}^{N}\right)}{ }^{\left(\mathscr{H}_{0}^{1},\|\cdot\|_{\mathscr{H}}{ }^{1}\right)} \subseteq \mathscr{L}^{1}\left(\mathscr{X}_{\mathscr{T}}^{N}\right) \subseteq \mathscr{L}^{1}\left(\mathscr{X}_{\mathscr{T}}\right),
$$

the last inclusion being true because $\mathscr{L}^{1}\left(\mathscr{X}_{\mathscr{T}}\right)$ is a stable subspace of $\left(\mathscr{H}_{0}^{1},\|\cdot\|_{\mathscr{H}^{1}}\right)$. The sequence $(N)_{N \in \mathbb{N}}$ localizes $X^{(f)}$ to $\mathscr{H}_{0}^{2}$ hence to $\mathscr{H}_{0}^{1}$. Therefore Lemma 3.4.1 yields

$$
\mathscr{L}^{1}\left(\left\{X^{(f)^{N}}, N \in \mathbb{N}\right\}\right)=\mathscr{L}^{1}\left(X^{(f)}\right) .
$$

Consequently,

$$
\mathscr{L}^{1}\left(\mathrm{~W}^{\sigma}\right) \cup \bigcup_{f \in L^{2}(\nu)} \mathscr{L}^{1}\left(X^{(f)}\right) \subseteq \mathscr{L}^{1}\left(\mathscr{X}_{\mathscr{T}}\right)
$$

By Theorem 1.4.5.(ii), the left-hand side of (3.9) is total in $\mathscr{L}^{1}(\mathscr{X})$ and, since $\mathscr{L}^{1}\left(\mathscr{X}_{\mathscr{T}}\right)$ is linear and closed in $\left(\mathscr{H}_{0}^{1},\|\cdot\|_{\mathscr{H}^{1}}\right)$, we conclude

$$
\mathscr{L}^{1}(\mathscr{X}) \subseteq \mathscr{L}^{1}\left(\mathscr{X}_{\mathscr{T}}\right) \subseteq \mathscr{H}_{0}^{1} .
$$

Theorem 3.3.2 yields $\mathscr{H}_{0}^{1}=\mathscr{L}^{1}(\mathscr{X})$ and hence, from the previous inclusions, $\mathscr{H}_{0}^{1}=$ $\mathscr{L}^{1}\left(\mathscr{X}_{\mathscr{T}}\right)$.

Thanks to Theorem 3.4.2 we can show that, given a Lévy process $\left(L, \mathbb{F}^{L}\right)$ it is always possible to find a countable system of martingales with the $\mathscr{H}^{1}$-PRP. This because the Hilbert space $L^{2}(\nu)$ is always separable.

3.4.3 Corollary. Let $\left(L, \mathbb{F}^{L}\right)$ be a Lévy process with characteristics $\left(\beta, \sigma^{2}, \nu\right)$. There always exists a countable family $\mathscr{X}_{\mathscr{T}}$ with the $\mathscr{H}^{1}$-PRP.

Proof. The Hilbert space $L^{2}(\nu)$ is separable because $\mathscr{B}(\mathbb{R})$ is a separable $\sigma$-algebra. Therefore we can find a system $\mathscr{T} \subseteq L^{2}(\nu)$ which is countable and total. The family $\mathscr{X}_{\mathscr{T}}$ associated with $\mathscr{T}$ is countable and, from Theorem 3.4.2, it follows that it has the $\mathscr{H}^{1}$-PRP. 
If $\mathscr{T} \subseteq L^{2}(\nu)$ is a complete orthogonal system, i.e., a total system of orthogonal functions, the family $\mathscr{X}_{\mathscr{T}}$ is a family of orthogonal martingales possessing the the $\mathscr{H}^{1}$-PRP (cf. Theorem 2.2.16.(vi) and Theorem 3.4.2). In conclusion the following corollary to Theorem 3.4.2 holds.

3.4.4 Corollary. Let $\mathscr{T} \subseteq L^{2}(\nu)$ be a complete orthogonal system. Then the family $\mathscr{X}_{\mathscr{T}}$ is a countable family of orthogonal martingales with the $\mathscr{H}^{1}$-PRP.

Because of Corollary 3.4.4, we can state that, if $\mathscr{T} \subseteq L^{2}(\nu)$ is a total system, any orthogonalization $\tilde{\mathscr{T}}$ of it leads to a family $\mathscr{X}_{\tilde{T}}$ of orthogonal martingales with the $\mathscr{H}^{1}$-PRP. Notice that Theorem 3.4.2 is not a characterization of the $\mathscr{H}^{1}$-PRP. It is therefore of interest to try to give some conditions on a system $\mathscr{T} \subseteq L^{2}(\nu)$ to ensure that its totality in $L^{2}(\nu)$ is also necessary for the family $\mathscr{X}_{\mathscr{T}}$ to have the $\mathscr{H}^{1}$-PRP with respect to $\mathbb{F}^{L}$. The following proposition shows that this is the case if, for example, $\mathscr{T} \subseteq L^{2}(\nu)$ is a finite system. We were not able to solve the problem for a more general system $\mathscr{T} \subseteq L^{2}(\nu)$.

3.4.5 Proposition. Let $\mathscr{T} \subseteq L^{2}(\nu)$ be a finite system. Then $\mathscr{X}_{\mathscr{T}}$ has the $\mathscr{H}^{1}-P R P$ if and only if $\mathscr{T}$ is total.

Proof. It is sufficient to prove that if $\mathscr{X}_{\mathscr{T}}$ has the $\mathscr{H}^{1}$-PRP and $\mathscr{T}$ is a finite system, then $\mathscr{T}$ is total in $L^{2}(\nu)$. The converse implication is a consequence Theorem 3.4.2. We assume that $\mathscr{T} \subseteq L^{2}(\nu)$ is a finite system and that $\mathscr{X}_{\mathscr{T}}$ has the $\mathscr{H}^{1}$-PRP and show that $\mathscr{T}$ is total in $L^{2}(\nu)$. The system $\mathscr{T}$ is finite and hence $\mathscr{X}_{\mathscr{T}}$ is a finite family of martingales. By assumption, $\mathscr{X}_{\mathscr{T}}$ possesses the $\mathscr{H}^{1}$-PRP, hence it satisfies condition $C_{\infty}$ (cf. Definition 1.4.10 and Theorem 1.4.11.(i)). But then $\mathscr{X}_{\mathscr{T}}$ satisfies also condition $C_{1}$, because it is a finite family of martingales satisfying condition $C_{\infty}$ (cf. Jacod (1979), Proposition 4.67). Hence, recalling Jacod (1979), Proposition 4.10, $\mathscr{X}_{\mathscr{T}}$ satisfies condition $C_{2}$. Let now $h \in L^{2}(\nu)$ be an orthogonal function to the closure in $L^{2}(\nu)$ of $\operatorname{Span}(\mathscr{T})$. The martingale $X^{(h)} \in \mathscr{H}_{\mathrm{loc}, 0}^{2}$ is orthogonal to $\mathscr{X}_{\mathscr{T}}$ (cf. Theorem 2.2.16.(vi)) and from condition $C_{2}$ we deduce $X^{(h)}=0$. As a consequence of Theorem 2.2.16.(v), we get $h=0$ which proves, in particular, that $\mathscr{T}$ is total in $L^{2}(\nu)$.

Although the possibility of finding a finite family $\mathscr{X}_{\mathscr{T}}$ with the $\mathscr{H}^{1}$-PRP is particularly important, especially for application, it is not difficult to recognize that this is possible only with a rather limited generality, as now we are going to verify.

3.4.6 Corollary. Let $\left(L, \mathbb{F}^{L}\right)$ be a Lévy process with characteristics $\left(\beta, \sigma^{2}, \nu\right)$. The following statements are equivalent:

(i) There exists a finite family $\mathscr{X}_{\mathscr{T}}$ possessing the $\mathscr{H}^{1}-P R P$ with respect to $\mathbb{F}^{L}$.

(ii) $L^{2}(\nu)$ is finite-dimensional.

(iii) $\nu$ has finite support.

Proof. The equivalence of (ii) and (iii) is obvious. Now we show that (ii) implies (i). If $L^{2}(\nu)$ is finite-dimensional, then there exists a finite system $\mathscr{T} \subseteq L^{2}(\nu)$ which is total. The family $\mathscr{X}_{\mathscr{T}}$ associated with such a system contains finitely many martingales and, from Theorem 3.4.2, it possesses the $\mathscr{H}^{1}$-PRP. Conversely, $\mathscr{X}_{\mathscr{T}}$ contains finitely many martingales if and only if $\mathscr{T} \subseteq L^{2}(\nu)$ is a finite system of 
functions. If we now assume that the finite family of martingales $\mathscr{X}_{\mathscr{T}}$ possesses the $\mathscr{H}^{1}$-PRP with respect to $\mathbb{F}^{L}$, by Proposition 3.4 .5 , it follows that $\mathscr{T}$ is total in $L^{2}(\nu)$. Hence $\mathscr{T} \subseteq L^{2}(\nu)$ is a finite total system in $L^{2}(\nu)$ and therefore $L^{2}(\nu)$ is finite-dimensional.

The situation discussed in Corollary 4.2.7 occurs, for example, if $L$ is a simple Lévy process, i.e., it is of the form

$$
L_{t}:=\mathrm{W}_{t}^{\sigma}+\sum_{j=1}^{m} \alpha_{j} N_{t}^{j}, \quad t \geq 0,
$$

where $\left(\mathrm{W}^{\sigma}, \mathbb{F}^{L}\right)$ is a Brownian motion with variance function $\left\langle\mathrm{W}^{\sigma}, \mathrm{W}^{\sigma}\right\rangle_{t}=\sigma^{2} t$, $\left(N^{j}, \mathbb{F}^{L}\right)$ is a homogeneous Poisson process with parameter $\lambda_{j}, j=1, \ldots, m$, and the vector $\left(\mathrm{W}^{\sigma}, N^{1}, \ldots, N^{m}\right)$ of processes is independent; $\alpha_{1}, \ldots, \alpha_{m}$ are real numbers. In this case $\nu\left(\left\{\alpha_{j}\right\}\right)=\lambda_{j}, j=1, \ldots, m$, and moreover we have $\operatorname{supp}(\nu)=$ $\left\{\alpha_{1}, \ldots, \alpha_{m}\right\}$. For a simple Lévy process, $L^{2}(\nu)$ is isomorphic to $\mathbb{R}^{m}$, hence every basis of $\mathbb{R}^{m}$, in particular any orthogonal one, leads to a finite family of martingales with the PRP with respect to $\mathbb{F}^{L}$.

The case in which $\mathscr{T} \subseteq L^{2}(\nu)$ is a finite total system of bounded functions is specially interesting because of the following proposition.

3.4.7 Proposition. Let $\mathscr{T} \subseteq L^{2}(\nu)$ be a finite total system of bounded functions. Then the associated family $\mathscr{X}_{\mathscr{T}}$ is contained in $\mathscr{H}_{\mathrm{loc}, 0}^{\infty}$ and it possesses the $\mathscr{H}^{q}-P R P$ with respect to $\mathbb{F}^{L}$, for every $q \in[1,+\infty)$.

Proof. If $\mathscr{T} \subseteq L^{2}(\nu)$ is total, then $\mathscr{X}_{\mathscr{T}}$ has the $\mathscr{H}^{1}$-PRP. Therefore $\mathscr{X}_{\mathscr{T}}$ satisfies condition $C_{\infty}$. Because $\mathscr{X}_{\mathscr{T}}$ is a finite system, from Jacod (1979), Proposition 4.67, we can deduce that it satisfies also condition $C_{1}$. But then, from Jacod (1979), Proposition $4.10, \mathscr{X}_{\mathscr{T}}$ satisfies also condition $C_{q}$, for every $q \in[1,+\infty]$. From Theorem 2.2.16.(iv), we know that $\mathscr{X}_{\mathscr{T}}$ is contained in $\mathscr{H}_{\mathrm{loc}, 0}^{\infty}$ and hence in $\mathscr{H}_{\mathrm{loc}, 0}^{q}$, for every $q \in[1,+\infty]$. Then $\mathscr{X}_{\mathscr{T}}$ is a family contained $\mathscr{H}_{\text {loc, } 0}^{q}$ which satisfied condition $C_{p}$, where $p$ is the conjugate exponent of $q$, for every $q$. The assert of the proposition follows immediately from Theorem 1.4.11.(ii).

\subsection{Square Integrable Lévy Processes}

In Theorem 3.4.2, given a Lévy process $\left(L, \mathbb{F}^{L}\right)$ with characteristics $\left(\beta, \sigma^{2}, \nu\right)$, we obtained that if $\mathscr{T} \subseteq L^{2}(\nu)$ is a total system, then the family $\mathscr{X}_{\mathscr{T}}$ defined by

$$
\mathscr{X}_{\mathscr{T}}:=\left\{\mathrm{W}^{\sigma}\right\} \cup\left\{X^{(f)}, \quad f \in \mathscr{T}\right\}
$$

possesses the $\mathscr{H}^{1}$-PRP with respect to $\mathbb{F}^{L}$. To prove this result we have showed in a first step that the bigger family

$$
\mathscr{X}:=\left\{\mathrm{W}^{\sigma}\right\} \cup\left\{X^{(f)}, \quad f \in L^{2}(\nu)\right\}
$$

possesses the $\mathscr{H}^{1}$-PRP with respect to $\mathbb{F}^{L}$. Although both $\mathscr{X}$ and $\mathscr{X}_{\mathscr{T}}$ were constructed starting from the structure of the Lévy process, $L$ does not appear in 
them explicitly. In this section we show that, under further regularity assumptions on the Lévy process $L$, we can replace, roughly speaking, the Gaussian part $\mathrm{W}^{\sigma}$ by $L$ itself. Thereafter, we assume that $L$ is square integrable, i.e., that $L_{t} \in L^{2}(\mathbb{P})$ for every $t \geq 0$. According to Theorem 2.3.8.(ii) this is equivalent to require that the function $1_{\{|x|>1\}} x$ belongs to $L^{2}(\nu)$, i.e., that $x \in L^{2}(\nu)$. For a square integrable Lévy process $\left(L, \mathbb{F}^{L}\right)$ we define the process $\bar{L}:=\left(\bar{L}_{t}\right)_{t \geq 0}$ by

$$
\bar{L}_{t}:=L_{t}-\mathbb{E}\left[L_{t}\right], \quad t \geq 0,
$$

which is an $\mathbb{F}^{L}$-martingale because of Lemma 2.1.1. By $\overline{\mathscr{X}}$ we designate the following family of martingales in $\mathscr{H}_{\mathrm{loc}, 0}^{2}$ :

$$
\overline{\mathscr{X}}:=\{\bar{L}\} \cup\left\{X^{(f)}, \quad f \in L^{2}(\nu)\right\} .
$$

Let $\mathscr{T} \subseteq L^{2}(\nu)$. We put

$$
\overline{\mathscr{X}}_{\mathscr{T}}:=\{\bar{L}\} \cup\left\{X^{(f)}, \quad f \in \mathscr{T}\right\} .
$$

We want to prove that, if $\mathscr{T} \subseteq L^{2}(\nu)$ is total in $L^{2}(\nu)$, then $\overline{\mathscr{X}}_{\mathscr{T}}$ possesses the $\mathscr{H}^{1}$ PRP with respect to $\mathbb{F}^{L}$. The first step that we do is to prove that the family $\overline{\mathscr{X}}$ possesses the $\mathscr{H}^{1}$-PRP. To this aim we show that all the solutions of the martingale problem associated with $\overline{\mathscr{X}}$ which are equivalent to $\mathbb{P}$ on $\left(\Omega, \tilde{\mathscr{F}}_{\infty}^{L}\right)$, say $\mathscr{Q}_{e}(\overline{\mathscr{X}})$, belong to $\mathscr{Q}_{e}(\mathscr{X})$ (cf. (3.4)). In other words, we put

$$
\mathscr{Q}_{e}(\overline{\mathscr{X}}):=\left\{\mathbb{Q} \in \mathscr{Q}(\overline{\mathscr{X}}): \mathbb{Q} \sim \mathbb{P} \text { on } \tilde{\mathscr{F}}_{\infty}^{L}\right\},
$$

where

$$
\mathscr{Q}(\overline{\mathscr{X}}):=\left\{\mathbb{Q} \text { on }\left(\Omega, \tilde{\mathscr{F}}_{\infty}^{L}\right) \text { such that } \overline{\mathscr{X}} \subseteq \mathscr{M}_{\mathrm{loc}, 0}\left(\mathbb{Q}, \mathbb{F}_{+}^{L}\right)\right\},
$$

and we prove that $\mathscr{Q}_{e}(\overline{\mathscr{X}})=\mathscr{Q}_{e}(\mathscr{X})=\{\mathbb{P}\}$, the last equality being true because of Theorem 3.3.1. Notice that $\mathbb{P} \in \mathscr{Q}(\overline{\mathscr{X}}) \cap \mathscr{Q}_{e}(\overline{\mathscr{X}})$ and therefore $\mathscr{Q}(\overline{\mathscr{X}}) \neq \emptyset \neq \mathscr{Q}_{e}(\overline{\mathscr{X}})$.

3.5.1 Theorem. Let $\left(L, \mathbb{F}^{L}\right)$ be a square integrable Lévy process on the probability space $\left(\Omega, \mathscr{F}_{\infty}^{L}, \mathbb{P}\right)$ with characteristic triplet $\left(\beta, \sigma^{2}, \nu\right)$ and Itô-Lévy decomposition

$$
L_{t}=\beta t+\mathrm{W}_{t}^{\sigma}+\mathrm{M}\left(1_{[0, t] \times\{|x|>1\}} x\right)+\overline{\mathrm{M}}\left(1_{[0, t] \times\{|x| \leq 1\}} x\right), \quad t \geq 0, \quad \mathbb{P} \text {-a.s. }
$$

where $\mathrm{W}^{\sigma}$ is the Gaussian part of $L$ and $\mathrm{M}$ its the Poisson random measure. Then

$$
\mathscr{Q}_{e}(\overline{\mathscr{X}})=\{\mathbb{P}\} .
$$

In particular, $\overline{\mathscr{X}}$ possesses the $\mathscr{H}^{1}-P R P$ with respect to $\mathbb{F}^{L}$.

Proof. By Theorem 3.3.1 we have $\mathscr{Q}_{e}(\mathscr{X})=\{\mathbb{P}\}$. We need only to prove that every element of $\mathscr{Q}_{e}(\overline{\mathscr{X}})$ belongs to $\mathscr{Q}_{e}(\mathscr{X})$. From the Itô-Lévy decomposition of $L$ and Lemma 2.2 .9 , it follows that $\mathbb{E}\left[L_{t}\right]=t\left(\beta+\nu\left(1_{\{|x|>1\}} x\right)\right)$. Moreover, by the square integrability of $L$, we have $x \in L^{2}(\nu)$, so that we can define the martingale $X^{(x)}$ and it belongs to $\overline{\mathscr{X}}$. We can write the Itô-Lévy decomposition of $\bar{L}$ as it follows:

$$
\bar{L}=\mathrm{W}^{\sigma}+X^{(x)}, \quad \mathbb{P} \text {-a.s. }
$$


We consider $\mathbb{Q}$ belonging to $\mathscr{Q}_{e}(\overline{\mathscr{X}})$. Because of the equivalence of $\mathbb{P}$ and $\mathbb{Q}$ we have

$$
\bar{L}=\mathrm{W}^{\sigma}+X^{(x)}, \quad \mathbb{Q} \text {-a.s. }
$$

To show that $\mathbb{Q} \in \mathscr{Q}_{e}(\mathscr{X})$ it is enough to prove that the process $\mathrm{W}^{\sigma}$ is a $\mathbb{Q}$-local martingale. But this is immediate from (3.14). Indeed, $\bar{L}, X^{(x)} \in \overline{\mathscr{X}}$ and they are $\mathbb{Q}$-local martingales because $\mathbb{Q} \in \mathscr{Q}_{e}(\overline{\mathscr{X}})$. From

$$
\mathrm{W}^{\sigma}:=\bar{L}-X^{(x)}, \quad \mathbb{Q} \text {-a.s. }
$$

we can deduce that also $\mathrm{W}^{\sigma}$ is a $\mathbb{Q}$-local martingale. Hence we have the inclusion $\mathscr{Q}_{e}(\overline{\mathscr{X}}) \subseteq \mathscr{Q}_{e}(\mathscr{X})=\{\mathbb{P}\}$. An application of Theorem 1.4.12 yields

$$
\mathscr{L}^{1}(\overline{\mathscr{X}})=\mathscr{H}_{0}^{1}\left(\mathbb{F}^{L}\right)
$$

and the proof is finished.

We remark that we could have shown Theorem 3.5 .1 by verifying that $\mathscr{L}^{1}\left(\mathrm{~W}^{\sigma}\right) \subseteq$ $\mathscr{L}^{1}(\overline{\mathscr{X}})$. This is the approach that we shall use in Chapter $4, \S 4.2 .2$.

Now we state an analogue of Theorem 3.4.2 for $\overline{\mathscr{X}}_{\mathscr{T}}$. The proof of this result is similar to the proof of Theorem 3.4.2 and we omit it.

3.5.2 Theorem. Let $\left(L, \mathbb{F}^{L}\right)$ be a square integrable Lévy process. The family $\overline{\mathscr{X}}_{\mathscr{T}}$ defined in (3.13) has the $\mathscr{H}^{1}-P R P$ with respect to $\mathbb{F}^{L}$ if $\mathscr{T}$ is total in $L^{2}(\nu)$.

We want to establish a result similar to Proposition 3.4.5. Now the situation is slightly different. The main point is that if we consider a function $h \in L^{2}(\nu)$ which is orthogonal to $\mathscr{T} \subseteq L^{2}(\nu)$, it is not true, in general, that the martingale $X^{(h)}$ is orthogonal to $\overline{\mathscr{X}}_{\mathscr{T}}$, because it could happen that $X^{(h)}$ is not orthogonal to the martingale $\bar{L}$.

3.5.3 Proposition. Let $\left(L, \mathbb{F}^{L}\right)$ be a square integrable Lévy process and $\mathscr{T} \subseteq L^{2}(\nu)$ be a finite system. Then $\overline{\mathscr{X}}_{\mathscr{T}}$ has the $\mathscr{H}^{1}-P R P$ and $x \in \operatorname{Span}(\mathscr{T})$ if and only if $\mathscr{T}$ is total.

Proof. It is enough to prove that if $\overline{\mathscr{X}}_{\mathscr{T}}$ is a finite family with the $\mathscr{H}^{1}$-PRP and $x \in \operatorname{Span}(\mathscr{T})$, then $\mathscr{T}$ is total in $L^{2}(\nu)$. Indeed, the converse implication follows from Theorem 3.5.2 and the fact that, because of $x \in L^{2}(\nu)$, if $\mathscr{T} \subseteq L^{2}(\nu)$ is a finite total system then clearly $x \in \operatorname{Span}(\mathscr{T})$. We assume that $\overline{\mathscr{X}}_{\mathscr{T}}$ is a finite family of martingales with the $\mathscr{H}^{1}$-PRP and that $x \in \operatorname{Span}(\mathscr{T})$. We show that the finite system $\mathscr{T} \subseteq L^{2}(\nu)$ is total. Because the family $\overline{\mathscr{X}}_{\mathscr{T}}$ is finite and it possesses the $\mathscr{H}^{1}$-PRP, condition $C_{\infty}$ (cf. Definition 1.4.10) is satisfied. From Jacod (1979), Proposition 4.67 we know that condition $C_{1}$ is satisfied. Hence condition $C_{2}$ is satisfied (cf. Jacod (1979), Proposition 4.10). Let now $h \in L^{2}(\nu)$ be orthogonal to $\overline{\operatorname{Span}(\mathscr{T})}{ }^{L^{2}(\nu)}=\operatorname{Span}(\mathscr{T})$, the equality being true because $\mathscr{T}$ is finite. The function $x$ belongs by assumption to $\operatorname{Span}(\mathscr{T})$ and so $h \perp x$. Therefore the martingale $X^{(h)} \in \mathscr{H}_{\text {loc }, 0}^{2}$ is orthogonal to $\overline{\mathscr{X}}_{\mathscr{T}}$ and from condition $C_{2}$ we deduce $X^{(h)}=0$. As a consequence of Theorem 2.2.16.(v), we get $h=0$ and this means that $\mathscr{T}$ is total in $L^{2}(\nu)$. 
We conclude this section by establishing the analogue of Corollary 3.4.6 for the system $\overline{\mathscr{X}}_{\mathscr{T}}$.

3.5.4 Corollary. Let $(L, \mathbb{F})$ be a square integrable Lévy process with Lévy measure $\nu$. The following statements are equivalent:

(i) There exists a finite family $\overline{\mathscr{X}}_{\mathscr{T}}$ with the $\mathscr{H}^{1}-P R P$ with respect to $\mathbb{F}^{L}$.

(ii) $L^{2}(\nu)$ is finite-dimensional.

(iii) $\nu$ has finite support.

Proof. The equivalence of (ii) and (iii) is obvious. If $L^{2}(\nu)$ is finite dimensional there exists a system $\mathscr{T}$ which is finite and total. From Theorem 3.5.2 we get that $\overline{\mathscr{X}}_{\mathscr{T}}$ is a finite family with the $\mathscr{H}^{1}$-PRP and so (ii) implies (i). For the converse implication we recall that $x \in L^{2}(\nu)$ because $L$ is square integrable. If the finite family $\overline{\mathscr{X}}_{\mathscr{T}}$ has the $\mathscr{H}^{1}$-PRP, then the family $\overline{\mathscr{X}}_{\mathscr{T}^{x}} \supseteq \overline{\mathscr{X}}_{\mathscr{T}}$ has the $\mathscr{H}^{1}$-PRP, where $\mathscr{T}^{x}:=\mathscr{T} \cup\{x\}$. Therefore, by Proposition 3.5.3, $\mathscr{T}^{x}$ must be total in $L^{2}(\nu)$ and so $L^{2}(\nu)$ is finite dimensional because $\mathscr{T}^{x}$ is a finite total system.

We notice that for the family $\overline{\mathscr{X}}_{\mathscr{T}}$ the analogue of Proposition 3.4 .7 cannot be proven unless the Lévy process $\left(L, \mathbb{F}^{L}\right)$ is locally bounded.

\subsection{Open Problems}

Let $\left(L, \mathbb{F}^{L}\right)$ be a Lévy process on the probability space $\left(\Omega, \mathscr{F}_{\infty}^{L}, \mathbb{P}\right)$ with characteristic triplet $\left(\beta, \sigma^{2}, \nu\right)$. In this section we discuss two problems which, in our opinion, are the main open problems related to the $\mathscr{H}^{1}$-PRP with respect to $\mathbb{F}^{L}$. The first problem concerns the possibility to give a characterization of the $\mathscr{H}^{1}$-PRP, i.e., to prove the converse of Theorem 3.4.2. The second problem is if in some cases the $\mathscr{H}^{1}$-PRP can be simplified in such a way to represent every $\mathscr{H}_{0}^{1}$-martingales as a series of stochastic integrals converging in $\left(\mathscr{H}_{0}^{1},\|\cdot\|_{\mathscr{H}^{1}}\right)$.

\subsubsection{Characterization of the Predictable Representation Property}

Let $\left(L, \mathbb{F}^{L}\right)$ be a Lévy process with characteristics $\left(\beta, \sigma^{2}, \nu\right)$. In Chapter 4 we shall prove that if $\mathscr{T}$ is a system of functions in $L^{2}(\nu)$, then the family

$$
\mathscr{X}_{\mathscr{T}}:=\left\{\mathrm{W}^{\sigma}\right\} \cup\left\{X^{(f)}, f \in \mathscr{T}\right\}
$$

possesses the $\mathscr{H}^{2}$-PRP with respect to $\mathbb{F}^{L}$ if and only if the system $\mathscr{T}$ is total in $L^{2}(\nu)$. In other words, we shall give a characterization of the $\mathscr{H}^{2}$-PRP for the family of martingales $\mathscr{X}_{\mathscr{T}}$ in terms of the system of functions $\mathscr{T}$. It was not possible for us to prove a similar result for the $\mathscr{H}^{1}$-PRP. Indeed, we only showed that if $\mathscr{T}$ is a total system in $L^{2}(\nu)$, then $\mathscr{X}_{\mathscr{T}}$ possesses the $\mathscr{H}^{1}$-PRP with respect to $\mathbb{F}^{L}$ but not the converse statement. What is the difference between the $\mathscr{H}^{1}$-case and the $\mathscr{H}^{2}$-case? The family $\mathscr{X}_{\mathscr{T}}$ consists of true martingales and is contained in $\mathscr{H}_{\mathrm{loc}, 0}^{2}$. If we assume that $\mathscr{X}_{\mathscr{T}}$ possesses the $\mathscr{H}^{2}$-PRP and we consider a function $h \in L^{2}(\nu)$ such that

$$
h \perp \overline{\operatorname{Span}(\mathscr{T})}^{L^{2}(\nu)},
$$


because of Theorem 2.2.16.(vi), we obtain that the martingale $X^{(h)}$ associated with $h$ is orthogonal to $\mathscr{X}_{\mathscr{T}}$. But $\mathscr{X}_{\mathscr{T}}$ satisfies condition $C_{2}$, because, by assumption, it possesses the $\mathscr{H}^{2}$-PRP (cf. Theorem 1.4.11). Hence $X^{(h)}=0$ and Theorem 2.2.16.(v) implies $h=0$. If we assume that $\mathscr{X}_{\mathscr{T}}$ has the $\mathscr{H}^{1}$-PRP, we can only conclude that it satisfies condition $C_{\infty}$, i.e., that every locally bounded martingale which is orthogonal to $\mathscr{X}_{\mathscr{T}}$ is evanescent. From this we cannot deduce as before that $\mathscr{T}$ is total. Indeed, if $h$ is an arbitrary function which is orthogonal to the closure in $L^{2}(\nu)$ of $\operatorname{Span}(\mathscr{T})$, we cannot conclude, in general, that it is also a bounded function. If it were the case, the associated martingale $X^{(h)}$ would be locally bounded and orthogonal to $\mathscr{X}_{\mathscr{T}}$ (cf. Theorem 2.2.16) and by condition $C_{\infty}$ and Theorem 2.2.16, we could conclude that $h=0$. Note that if the system $\mathscr{T}$ is a finite total system, in Proposition 3.4.5 we gave the desired characterization for the $\mathscr{H}^{1}$-PRP in terms of the totality of the system $\mathscr{T}$ in $L^{2}(\nu)$. For this we used that, for finite families of martingales, all the conditions $C_{q}$ are equivalent.

It is clear that to give a characterization of the $\mathscr{H}^{1}$-PRP in the general case of a family $\mathscr{X}_{\mathscr{T}}$ which is not finite, the approach which goes trough the conditions $C_{q}$ cannot be followed. The relevant questions are then: Is it true that the totality of $\mathscr{T}$ is necessary for $\mathscr{X}_{\mathscr{T}}$ to have the $\mathscr{H}^{1}$-PRP? If not, how to construct a counterexample? If yes, in which way should we prove it?

\subsubsection{A Stronger Representation}

Let $\left(L, \mathbb{F}^{L}\right)$ be a Lévy process with characteristic triplet $\left(\beta, \sigma^{2}, \nu\right)$. We consider a countable system $\mathscr{T}:=\left\{f_{1}, f_{2}, \ldots\right\} \subseteq L^{2}(\nu)$ which is total in $L^{2}(\nu)$. This is always possible because of the separability of $L^{2}(\nu)$. To simplify the notations, we denote the family of martingales associated with $\mathscr{T}$ by $\mathscr{X}_{\mathscr{T}}:=\left\{X^{n}, n \geq 0\right\}$, where $X^{0}:=\mathrm{W}^{\sigma}$ and $X^{n}:=X^{\left(f_{n}\right)}, n \geq 1$. We know that $\mathscr{X}_{\mathscr{T}}$ has the $\mathscr{H}^{1}$-PRP with respect to $\mathbb{F}^{L}$, thus, every $M \in \mathscr{H}_{0}^{1}$ can be represented as

$$
M=\lim _{n \rightarrow+\infty}\left(\sum_{k=0}^{m_{n}} H^{n, k} \cdot X^{k}\right)
$$

where $H^{n, k} \in \mathrm{L}^{1}\left(X^{k}\right), n, k \geq 0$, and the limit in $(3.15)$ is taken in $\left(\mathscr{H}_{0}^{1},\|\cdot\|_{\mathscr{C}^{1}}\right)$. The meaning of (3.15) is that for every martingale $M \in \mathscr{H}_{0}^{1}$, there exists a sequence $\left(Y^{n}\right)_{n \geq 1}$,

$$
Y^{n} \in \operatorname{Span}\left(\bigcup_{n=0}^{\infty} \mathscr{L}^{1}\left(X^{n}\right)\right),
$$

converging in $\left(\mathscr{H}_{0}^{1},\|\cdot\|_{\mathscr{H}^{1}}\right)$ to $M$. The $\mathscr{H}^{1}$-PRP would become a stronger result if every martingale $M \in \mathscr{H}_{0}^{1}$ could be represented as a series of stochastic integrals, that is, if for every $M \in \mathscr{H}_{0}^{1}$

$$
M=\sum_{n=0}^{\infty} H^{n} \cdot X^{n}, \quad H^{n} \in \mathrm{L}^{1}\left(X^{n}\right), \quad n \geq 0 .
$$

We investigated if it is possible to construct a family of martingales $\mathscr{X}_{\mathscr{T}}$ having the $\mathscr{H}^{1}$-PRP with respect to $\mathbb{F}^{L}$ in the stronger form expressed in (3.16). In particular, 
we were looking for some conditions on the system $\mathscr{T} \subseteq L^{2}(\nu)$ to ensure (3.16). We were not able to give a positive answer to this question. The problem risen by (3.16) can be formulated in terms of stable subspaces, as it follows. We consider the linear space $\mathscr{C}^{1} \subseteq \mathscr{H}_{0}^{1}$ consisting of all the martingales in $\mathscr{H}_{0}^{1}$ which can be represented as in (3.16), i.e.,

$$
\mathscr{C}^{1}:=\left\{M \in \mathscr{H}_{0}^{1} \text { such that }(3.16) \text { holds }\right\} .
$$

It is evident that $\mathscr{C}^{1}$ is a stable-under-stopping subset of $\mathscr{L}^{1}\left(\mathscr{X}_{\mathscr{T}}\right)$. Is $\mathscr{C}^{1}$ also closed in $\left(\mathscr{H}_{0}^{1},\|\cdot\|_{\mathscr{H}^{1}}\right)$ ? Or, in other words, is $\mathscr{C}^{1}$ a stable subspace of $\mathscr{H}_{0}^{1}$ ? The system $\mathscr{X}_{\mathscr{T}}$ is contained in $\mathscr{H}_{\text {loc }, 0}^{2}$ and hence in $\mathscr{H}_{\text {loc }, 0}^{1}\left(=\mathscr{M}_{\text {loc }, 0}\right)$. Clearly, $\mathscr{C}^{1}$ contains the set

$$
\mathscr{H}:=\left\{\left(X^{n}\right)^{T}: T \text { stopping time such that }\left(X^{n}\right)^{T} \in \mathscr{H}_{0}^{1}, n \geq 0\right\}
$$

and therefore, if $\mathscr{C}^{1}$ were a stable subspace, it would yield

$$
\mathscr{C}^{1}=\mathscr{L}^{1}\left(\mathscr{X}_{\mathscr{T}}\right)
$$

because $\mathscr{L}^{1}\left(\mathscr{X}_{\mathscr{T}}\right)$ is the smallest stable subspace of $\mathscr{H}_{0}^{1}$ containing $\mathscr{H}$ (cf. Lemma 1.4.4). In Protter (2005), in the proof of Theorem IV.4.57, it is asserted that the space $\mathscr{C}^{1}$ is in fact closed in $\left(\mathscr{H}_{0}^{1},\|\cdot\|_{\mathscr{H}^{1}}\right)$ but neither we could understand the proof of this claim proposed in Protter (2005), nor we were able to show it autonomously.

The situation in $\mathscr{H}_{0}^{2}$ is simpler and we sketch it shortly. Let $\mathscr{T} \subseteq L^{2}(\nu)$ be an orthogonal system and $\mathscr{X}_{\mathscr{T}}$ the associated family of strongly orthogonal martingales. If instead of $\mathscr{C}^{1}$ we consider the set $\mathscr{C}^{2}$ of all the martingales belonging to $\mathscr{H}_{0}^{2}$ for which the analogue in $\mathscr{H}_{0}^{2}$ of (3.16) holds, it is an easy task to show that $\mathscr{C}^{2}$ is closed in $\left(\mathscr{H}_{0}^{2},\|\cdot\|_{\mathscr{C}^{2}}\right)$ (cf. Lemma 1.4.7). This because the strong orthogonality of the martingales $\mathscr{X}_{\mathscr{T}}$ passes to the stable subspace $\mathscr{L}^{2}\left(X^{n}\right), n \geq 0$. The strong orthogonality of the spaces $\mathscr{L}^{2}\left(X^{n}\right), n \geq 0$, implies that they are closed orthogonal subspaces in the sense of the Hilbert space $\left(\mathscr{H}_{0}^{2},\|\cdot\|_{2}^{*}\right)$. We recall that $\|\cdot\|_{2}^{*}$ is the norm induced by the scalar product and that, thanks to DBG's inequality (cf. Theorem (1.23)), it is equivalent to the norm $\|\cdot\|_{\mathscr{H}^{2}}$. From Theorem 1.4.8 it follows that

$$
\mathscr{L}^{2}\left(\mathscr{X}_{\mathscr{T}}\right)=\bigoplus_{n=0}^{\infty} \mathscr{L}^{2}\left(X^{n}\right)=: \mathscr{C}^{2}
$$

Therefore, assuming to know that the totality of $\mathscr{T}$ is sufficient for $\mathscr{X}_{\mathscr{T}}$ to have the $\mathscr{H}^{2}$-PRP, we can conclude that if $\mathscr{T}$ is a complete orthogonal system, then every martingale $M \in \mathscr{H}_{0}^{2}$ can be represented as

$$
M=\sum_{n=0}^{\infty} H^{n} \cdot X^{n}, \quad H^{n} \in \mathrm{L}^{2}(X n), \quad n \geq 0,
$$

where the previous series converges in $\left(\mathscr{H}_{0}^{2},\|\cdot\|_{2}^{*}\right)$ and hence, by the equivalence of the norms, in $\left(\mathscr{H}_{0}^{2},\|\cdot\|_{\mathscr{H}^{2}}\right)$. To obtain this representation, it seems that the orthogonality of the system $\mathscr{T} \subseteq L^{2}(\nu)$, and hence the strong orthogonality of the martingales $\mathscr{X}_{\mathscr{T}}$, is also a necessary condition. Indeed, if $\mathscr{T} \subseteq L^{2}(\nu)$ is a finite total 
system of functions, say $\mathscr{T}:=\left\{f_{1}, \ldots, f_{N}\right\}$, which are not orthogonal in $L^{2}(\nu)$ and we consider the space $\mathscr{L}^{2}\left(\mathscr{X}_{\mathscr{T}}\right)$, it is not true that

$$
\mathscr{L}^{2}\left(\mathscr{X}_{\mathscr{T}}\right)=\operatorname{Span}\left(\bigcup_{n=0}^{N} \mathscr{L}^{2}\left(X^{n}\right)\right)=: \mathscr{K} .
$$

Instead, we know that $\mathscr{L}^{2}\left(\mathscr{X}_{\mathscr{T}}\right)$ is equal to the closure in $\left(\mathscr{H}_{0}^{2},\|\cdot\|_{\mathscr{H}^{2}}\right)$ of $\mathscr{K}$ and, in general, $\mathscr{K}$ is not closed in $\left(\mathscr{H}_{0}^{2},\|\cdot\|_{\mathscr{H}^{2}}\right)($ cf. Jacod (1979), Exercise 4.6). This is due to the following fact. If $M=\left(M^{1}, \ldots, M^{m}\right), M^{i} \in \mathscr{H}_{\mathrm{loc}, 0}^{2}$, and $H=\left(H^{1}, \ldots, H^{m}\right)$, $H^{i} \in \mathrm{L}^{2}\left(M^{i}\right), i=1, \ldots m, m \geq 1$, it seems natural to define the stochastic integral of $H$ with respect to $M$ as the sum of the one-dimensional stochastic integrals of each component, i.e.,

$$
H \cdot M:=\sum_{i=1}^{m} H^{i} \cdot M^{i} .
$$

However, this is not the most general stochastic integral with respect to $M$. Rather, one should consider the multi-dimensional stochastic integral of $H$ with respect to $M$. We do not go here into details, but we refer to Jacod (1979), §4.2 and $\S 4.4$. and Jacod \& Shiryaev (2000), §III.4a. We only remark that if $\left\langle M^{i}, M^{j}\right\rangle=0, i \neq j$, $i, j=1, \ldots, m$, then the multi-dimensional stochastic integral of $H$ with respect to $M$ coincides with the integral introduced in (3.17).

The orthogonality of the system $\mathscr{T} \subseteq L^{2}(\nu)$ seems not to be sufficient to (3.16). Indeed, even in case of a complete orthogonal system $\mathscr{T} \subseteq L^{2}(\nu)$, and hence of an orthogonal family of martingales $\mathscr{X}_{\mathscr{T}}$, we cannot conclude that the spaces $\mathscr{L}^{1}\left(X^{n}\right)$, $n \geq 0$, are strongly orthogonal (cf. Lemma 1.4.6). On the other side, we cannot exclude that formula (3.16) is true and, because an opportune choice of the system $\mathscr{T}$ (say, e.g., a complete orthogonal system in $L^{2}(\nu)$ of bounded functions) leads to a family of martingales $\mathscr{X}_{\mathscr{T}}$ with very special properties, we can hope that there is the possibility to obtain (3.16) at least in some special cases. Formula (3.16) is particularly important for several reasons. First of all it is much simpler than (3.15) and so more convenient for applications. But in our opinion the real interest of this formula concerns the fact that, if it were true, it would be possible to construct families consisting of countably may martingales for which all the conditions $C_{q}$, $q \geq 1$, are equivalent. This would be a nice complement to Jacod (1979), Proposition 4.13 and 4.67. We now show an idea to prove that if $\mathscr{X}_{\mathscr{T}}$ has the $\mathscr{H}^{1}$-PRP in the form of (3.16), then, for some special system $\mathscr{T}, \mathscr{X}_{\mathscr{T}}$ satisfies condition $C_{1}$. We preliminary observe that if $M \in \mathscr{H}_{\mathrm{loc}, 0}^{2}$ is such that $\left.\langle M, M\rangle_{t}=c t, c\right\rangle 0$, then for every $H \in \mathrm{L}^{1}(M)$ such that $H=0 \lambda_{+} \otimes \mathbb{P}$-a.e., it follows $H \cdot M=0$. Indeed, if $M \in \mathscr{H}_{\mathrm{loc}, 0}^{2}$, then $[M, M] \in \mathscr{A}_{\mathrm{loc}}^{+}$and $\langle M, M\rangle$ is its compensator and therefore

$$
\mathbb{E}\left[[H \cdot M, H \cdot M]_{T}\right]=\mathbb{E}\left[H^{2} \cdot[M, M]_{T}\right]=\mathbb{E}\left[H^{2} \cdot\langle M, M\rangle_{T}\right]=c \mathbb{E}\left[\int_{0}^{T} H_{t}^{2} \mathrm{~d} t\right]=0 .
$$

This implies that $[H \cdot M, H \cdot M]$ is a martingale or, equivalently, that $H \cdot M$ is orthogonal to itself, hence evanescent.

Let $\mathscr{T} \subseteq L^{2}(\nu)$ be a complete orthogonal system of bounded functions. Then $\mathscr{X}_{\mathscr{T}}$ is a family of locally bounded and strongly orthogonal martingales (cf. Theorem 
2.2.16) with the $\mathscr{H}^{1}$-PRP. We claim that, if for $\mathscr{X}_{\mathscr{T}}$ the $\mathscr{H}^{1}$-PRP assumes the stronger form expressed in (3.16), then $\mathscr{X}_{\mathscr{T}}$ satisfies also condition $C_{1}$ and this would imply that all the conditions $C_{q}$ are equivalent. Therefore, as a consequence of Theorem 1.4.11, we could conclude that if $\mathscr{T} \subseteq L^{2}(\nu)$ is a complete orthogonal system of bounded functions, $\mathscr{X}_{\mathscr{T}}$ possess the $\mathscr{H}^{q}$-PRP, for every $q \in[1,+\infty)$.

Proof. Under the mentioned assumptions on $\mathscr{X}_{\mathscr{T}}$, we are going to show that all the martingales in $\mathscr{H}_{0}^{1}$ which are orthogonal to $\mathscr{X}_{\mathscr{T}}$ are evanescent. Let $M \in \mathscr{H}_{0}^{1}$ be such that $M \perp \mathscr{X}_{\mathscr{T}}$. Because $\mathscr{T}$ is total and we have assumed that it is such that the $\mathscr{H}^{1}$-PRP holds as in (3.16), we have

$$
M=\sum_{n=0}^{+\infty} H^{n} \cdot X^{n}, \quad H^{n} \in \mathrm{L}^{1}\left(X^{n}\right), \quad n \geq 0 .
$$

Now we fix $k>0$ and consider the martingale $X^{k}$. Because of Theorem 2.2.16.(iv), this is a locally bounded martingale and it is orthogonal to $M$ and, for every $n \neq k$, to $X^{n}$. There exists a sequence $\left(S_{j}\right)_{j \geq 1}$ of stopping times such that $S_{j} \uparrow+\infty$ as $j \rightarrow+\infty$ and $\left(X^{k}\right)^{S_{j}} \in \mathscr{H}_{0}^{\infty} \subseteq B M O_{0}$. So the process

$$
N^{j}:=\left(X^{k}\right)^{S_{j}}
$$

is in $B M O_{0}$ and is again orthogonal to $M$ and to $X^{n}$, for every $n \neq k$. Because $M \in \mathscr{H}_{0}^{1}$, we get that $\left[M, N^{j}\right]$ is a uniformly integrable martingale starting at 0 (cf. Lemma 1.4.6.(ii) with $H=1$ ). The same holds for $H^{n} \cdot\left[X^{n}, N^{j}\right]$, for every $n \neq k$. Hence, from Lemma 1.2.2, for every stopping time $T$,

$$
\mathbb{E}\left[\left[M, N^{j}\right]_{T}\right]=0, \quad \mathbb{E}\left[H^{n} \cdot\left[X^{n}, N^{j}\right]_{T}\right]=0, \quad n \neq k .
$$

Because of Lemma 1.3.1, the linear functional $\mathbb{E}\left[\left[\cdot, N^{j}\right]_{\infty}\right]$ is continuous on $\mathscr{H}_{0}^{1}$. This implies that also the linear functional $\mathbb{E}\left[\left[\cdot, N^{j}\right]_{T}\right]$ is continuous on $\mathscr{H}_{0}^{1}$, for every stopping time $T$. Hence, because the convergence in (3.18) takes place in $\left(\mathscr{H}_{0}^{1},\|\cdot\|_{\mathscr{H}^{1}}\right)$, we get

$$
\mathbb{E}\left[H^{k} \cdot\left[N^{j}, X^{k}\right]_{T}\right]=0, \quad T \text { stopping time. }
$$

By Lemma 1.4.6.(ii), $\left|H^{k}\right| \cdot \operatorname{Var}\left(\left[X^{k}, N^{j}\right]\right)$ is integrable and so, because of (3.19) and Lemma 1.2.2, we can conclude that $H^{k} \cdot\left[X^{k}, N^{j}\right]$ is a uniformly integrable martingale. For these reasons the compensator of $H^{k} \cdot\left[N^{j}, X^{k}\right]$ exists and is equal to zero. On the other side, because $\left\langle X^{k}, N^{j}\right\rangle$ is the dual predictable projection of $\left[X^{k}, N^{j}\right]$, we get that the compensator of $H^{k} \cdot\left[X^{k}, N^{j}\right]$ is $H^{k} \cdot\left\langle X^{k}, N^{j}\right\rangle$. So, $H^{k} \cdot\left\langle X^{k}, N^{j}\right\rangle=0$. Moreover, $\lambda_{+} \otimes \mathbb{P}$-a.e., we have

$$
\begin{aligned}
0=H^{k} \cdot\left\langle N^{j}, X^{k}\right\rangle_{t} & =H^{k} \cdot\left\langle X^{k^{S_{j}}}, X^{k}\right\rangle_{t} \\
& =H^{k} \cdot\left\langle X^{k}, X^{k}\right\rangle_{t}^{S_{j}} \\
& =H^{k} 1_{\left[0, S_{j}\right]} \cdot\left\langle X^{k}, X^{k}\right\rangle_{t} \\
& =\nu\left(f_{k}^{2}\right) \int_{0}^{t} H_{u}^{k} 1_{\left[0, S_{j}\right]}(u) \mathrm{d} u, \quad t \geq 0,
\end{aligned}
$$


and this implies $H^{k} 1_{\left[0, S_{j}\right]}=0 \lambda_{+} \otimes \mathbb{P}$-a.e., for every $j \geq 1$. But

$$
H_{t}^{k} 1_{\left[0, S_{j}\right]}(u) \longrightarrow H_{t}^{k}, \quad \text { pointwise as } j \rightarrow+\infty \text {. }
$$

Hence $H^{k}=0 \lambda_{+} \otimes \mathbb{P}$-a.e. and above we have observed that this implies $H^{k} \cdot X^{k}=0$. Because of the arbitrariness of $k>0$ and of (3.18), recalling that $X^{0}:=\mathrm{W}^{\sigma}$, we can conclude that $M$ is of the form

$$
M=\phi \cdot \mathrm{W}^{\sigma} .
$$

But then $M \in \mathscr{H}_{\mathrm{loc}, 0}^{\infty}$ hence $M^{S_{j}} \in B M O_{0}$, where now $\left(S_{j}\right)_{j \geq 1}$ is a sequence localizing $M$ to $\mathscr{H}_{0}^{\infty}$. Because of $M \perp \mathrm{W}^{\sigma}$ we have also $M^{S_{j}} \perp \mathrm{W}^{\sigma}$ and by Lemma 1.4.6 we get $M^{S_{j}} \perp \mathscr{L}^{1}\left(\mathrm{~W}^{\sigma}\right)$, for every $j \geq 1$. Hence, $M^{S_{j}}=0$ for every $j \geq 1$, because $M^{S_{j}} \in \mathscr{L}^{1}\left(\mathrm{~W}^{\sigma}\right)$ and so it is orthogonal to itself. Therefore, because of $M^{S_{j}} \longrightarrow M$ pointwise as $j \rightarrow+\infty$, we get $M=0$. We have then shown that $\mathscr{X}_{\mathscr{T}}$ satisfies condition $C_{1}$. Because of Jacod (1979), Proposition 4.10, this implies that $\mathscr{X}_{\mathscr{T}}$ satisfies also condition $C_{q}$, for every $q \in[1,+\infty]$.

We observe that if $\mathscr{T} \subseteq L^{2}(\nu)$ is a system of bounded functions such that $\mathscr{L}^{1}(\mathscr{X})=\mathscr{C}^{1}$, then $\mathscr{X}_{\mathscr{T}}$ has the $\mathscr{H}^{1}$-PRP, if and only if $\mathscr{T}$ is total in $L^{2}(\nu)$. Indeed, for such a system all the conditions $C_{q}, q \geq 1$, are equivalent and, in particular it satisfies also condition $C_{2}$. Note that, if $\mathscr{T} \subseteq L^{2}(\nu)$ is a finite total system of bounded functions, we have shown in Proposition 3.4.7 that the associated family of martingales $\mathscr{X}_{\mathscr{T}}$ possesses the $\mathscr{H}^{q}$-PRP with respect to $\mathbb{F}^{L}$, for every $q \geq 1$. However, also in this specially simple case, even if $\mathscr{T}$ is a system of orthogonal functions, we could not conclude that (3.16) holds.

Another important consequence of (3.16) concerns the uniqueness of the $\mathscr{H}^{1}$-PRP. More precisely, assuming (3.16), we were able to prove that the integrands appearing in (3.16) are $\lambda_{+} \otimes \mathbb{P}$-a.e. uniquely determined by the martingale $M$. To verify this fact it is enough to consider two representation of a martingale $M \in \mathscr{H}_{0}^{1}$, one with integrands $\left(H^{n}\right)_{n \geq 0}$ and one with integrands $\left(K^{n}\right)_{n \geq 0}$, and then, with similar ideas as in the proof discussed above, to prove that $H^{n}=K^{n}, \lambda_{+} \otimes \mathbb{P}$-a.e. 

CHAPTER 4

\section{Representation of Square Integrable Martingales}

Let $L$ be a Lévy process on the probability space $\left(\Omega, \mathscr{F}_{\infty}^{L}, \mathbb{P}\right)$. We recall that we put $\mathscr{F}_{\infty}^{L}:=\sigma\left(L_{t}, t \geq 0\right) \vee \mathscr{N}(\mathbb{P})$, where $\mathscr{N}(\mathbb{P})$ denotes the null-sets of $\mathbb{P}$ in $\sigma\left(L_{t}, t \geq 0\right)$. By $\mathbb{F}^{L}=\left(\mathscr{F}_{t}^{L}\right)_{t \geq 0}$ we designate the completion in $\mathscr{F}_{\infty}^{L}$ of the filtration generated by $L$, i.e., $\mathscr{F}_{t}^{L}:=\sigma\left(L_{s}, 0 \leq s \leq t\right) \vee \mathscr{N}(\mathbb{P}), t \geq 0$. By Proposition 2.1.4, we know that $\mathbb{F}^{L}$ coincides with the natural filtration of $L$, thus, it is the smallest filtration satisfying the usual conditions and making $L$ adapted. Furthermore, $L$ is a Lévy process relative to $\mathbb{F}^{L}$. We are going to discuss the problem of the $\mathscr{H}^{2}$-PRP with respect to $\mathbb{F}^{L}$. More precisely, we show how to construct families of locally square integrable $\mathbb{F}^{L}$-martingales whose generated stable subspace in $\mathscr{H}_{0}^{2}\left(\mathbb{F}^{L}\right)$ equals the space $\mathscr{H}_{0}^{2}\left(\mathbb{F}^{L}\right)$ itself. The most elegant way to tackle the problem is at first to consider a rather general case, outside of the frame of Lévy processes. For this purpose, in $\S 4.1$ we consider a family $\mathscr{Z}$ of locally square integrable martingales and we look for sufficient conditions to ensure that the stable subspace generated by $\mathscr{Z}$ in $\mathscr{H}_{0}^{2}$ equals $\mathscr{H}_{0}^{2}$ itself. The reference filtration is the smallest filtration satisfying the usual conditions with respect to which $\mathscr{Z}$ is a family of adapted local martingales. In $\S 4.2$ we apply the theory developed in $\S 4.1$ to solve the problem of finding a family of locally square integrable martingales possessing the $\mathscr{H}^{2}$-PRP in the special case of Lévy processes. We also analyze the particularly interesting situation in which the family of locally square integrable $\mathbb{F}^{L}$-martingales with the $\mathscr{H}^{2}$-PRP consists of orthogonal martingales. The procedure which we shall follow is different from the one that we saw in Chapter 3 for the $\mathscr{H}^{1}$-PRP. This is due to the fact that, if $q>1$, Theorem 1.4 .12 nothing says about the $\mathscr{H}^{q}$-PRP for a family $\mathscr{X} \subseteq \mathscr{M}_{\mathrm{loc}, 0}$ and therefore we cannot approach the problem of the $\mathscr{H}^{2}$-PRP by means of an appropriate martingale problem.

\subsection{Compensated-Covariation Stable Families}

We consider a complete probability space $(\Omega, \mathscr{F}, \mathbb{P})$ and a filtration $\mathbb{F}$ satisfying the usual conditions. Let $I$ be an arbitrary set of indexes and $\mathscr{Z} \subseteq \mathscr{H}_{\mathrm{loc}, 0}^{2}$ be a family 
of local martingales indexed on $I$, i.e.,

$$
\mathscr{Z}:=\left\{M^{(\alpha)}, \quad \alpha \in I\right\} .
$$

For two elements $M^{(\alpha)}$ and $M^{(\beta)}, \alpha, \beta \in I$, we define the process

$$
M^{(\alpha, \beta)}:=\left[M^{(\alpha)}, M^{(\beta)}\right]-\left\langle M^{(\alpha)}, M^{(\beta)}\right\rangle
$$

and we call it the compensated-covariation process. From Proposition 1.2.11.(ii) we know that the processes $\left[M^{(\alpha)}, M^{(\beta)}\right]$ and $\left\langle M^{(\alpha)}, M^{(\beta)}\right\rangle, \alpha, \beta \in I$, belong to $\mathscr{A}_{\text {loc }}$ and the predictable process $\left\langle M^{(\alpha)}, M^{(\beta)}\right\rangle$ is the compensator of $\left[M^{(\alpha)}, M^{(\beta)}\right]$. Therefore, in general, the process $M^{(\alpha, \beta)}$ belongs only to $\mathscr{M}_{\mathrm{loc}, 0}$ (cf. Theorem 1.2.4 and the comment following it) and not to $\mathscr{H}_{\mathrm{loc}, 0}^{2}$. This fact justifies the following definition.

4.1.1 Definition. Let $I$ be a family of indexes and $\mathscr{Z}:=\left\{M^{(\alpha)}, \alpha \in I\right\}$ a family of local martingales in $\mathscr{H}_{\text {loc }, 0}^{2}$ indexed on $I$.

(i) We say that $\mathscr{Z}$ is a compensated-covariation stable family if the compensatedcovariation process $M^{(\alpha, \beta)}$ (cf. (4.2)) belongs to $\mathscr{Z}$ for every $\alpha$ and $\beta$ in $I$.

(ii) Let $\mathscr{Z}$ be a compensated-covariation stable family and let $\alpha_{1}, \ldots, \alpha_{m} \in I$, $m \geq 2$. We define the process $M^{\left(\alpha_{1}, \ldots, \alpha_{m}\right)}$ recursively by

$$
M^{\left(\alpha_{1}, \ldots, \alpha_{m}\right)}:=\left[M^{\left(\alpha_{1}, \ldots, \alpha_{m-1}\right)}, M^{\left(\alpha_{m}\right)}\right]-\left\langle M^{\left(\alpha_{1}, \ldots, \alpha_{m-1}\right)}, M^{\left(\alpha_{m}\right)}\right\rangle .
$$

The reader should keep in mind that the process $M^{\left(\alpha_{1}, \ldots, \alpha_{m}\right)}$ is defined by (4.3) only for $m \geq 2$ and this does not cause ambiguity of notations with the case $m=1$. We note that, if the indexed family $\mathscr{Z}$ is a compensated-covariation stable family of local martingales, then the process $M^{\left(\alpha_{1}, \ldots, \alpha_{m}\right)}$ introduced in (4.3) is well defined for every $\alpha_{1}, \ldots, \alpha_{m}$. Indeed, by induction, it is immediate to see that for each $m \geq 2$ the processes $M^{\left(\alpha_{1}, \ldots, \alpha_{m-1}\right)}$ belongs to $\mathscr{Z}$, because $\mathscr{Z}$ is compensated-covariation stable. Hence $M^{\left(\alpha_{1}, \ldots, \alpha_{m-1}\right)}$ and $M^{\left(\alpha_{m}\right)}$ belong to $\mathscr{H}_{\text {loc }, 0}^{2}$ and we can introduce the process $M^{\left(\alpha_{1}, \ldots, \alpha_{m}\right)}$. It is obvious that if $\mathscr{Z}$ is a compensated-covariation stable family of $\mathscr{H}_{\text {loc }, 0}^{2}$, then the process $M^{\left(\alpha_{1}, \ldots, \alpha_{m}\right)}$ belongs again to $\mathscr{Z}$, for every $\alpha_{1}, \ldots, \alpha_{m}$ in $I$ and for every natural number $m \geq 2$. Therefore, for every $\alpha_{1}, \ldots, \alpha_{m} \in I, m \geq 2$, there exists an element $\gamma \in I$ such that $M^{\left(\alpha_{1}, \ldots, \alpha_{m}\right)}=M^{(\gamma)}$. Contrarily, if $\mathscr{Z}$ is not compensated-covariation stable, the process $M^{\left(\alpha_{1}, \alpha_{2}\right)}$ belongs only to $\mathscr{M}_{\mathrm{loc}, 0}$ and so we cannot introduce the process $M^{\left(\alpha_{1}, \ldots, \alpha_{m}\right)}$ for $m \geq 3$, because the predictable process $\left\langle M^{\left(\alpha_{1}, \ldots, \alpha_{m-1}\right)}, M^{\left(\alpha_{m}\right)}\right\rangle$ could be not defined.

For any family $\mathscr{Z} \subseteq \mathscr{H}_{\mathrm{loc}, 0}^{2}$ indexed on $I$ and any arbitrary collection of finitevalued stopping times $\mathscr{S}$, we define the family $\mathscr{Z}^{\mathscr{S}}$ by

$$
\mathscr{Z}^{\mathscr{S}}:=\left\{M^{(\alpha)^{T}}, \quad T \in \mathscr{S}, \quad \alpha \in I\right\} .
$$

It is clear that $\mathscr{Z}^{\mathscr{S}} \subseteq \mathscr{H}_{\text {loc }, 0}^{2}$. The following lemma is an easy consequence of the properties of the brackets $[\cdot, \cdot \cdot]$ and $\langle\cdot, \cdot\rangle$.

4.1.2 Lemma. Let $\mathscr{Z}$ be a compensated-covariation stable family of $\mathscr{H}_{\mathrm{loc}, 0}^{2}$ and $\mathscr{S}$ a family of finite-valued stopping times such that $T \wedge S \in \mathscr{S}$ for every $T$ and $S$ belonging to $\mathscr{S}$. The family $\mathscr{Z}^{\mathscr{S}}$ introduced in (4.4) is a compensated-covariation stable family of local martingales in $\mathscr{H}_{\mathrm{loc}, 0}^{2}$. 
Proof. If $M \in \mathscr{H}_{\mathrm{loc}, 0}^{2}$ and $T$ is a finite-valued stopping time, the process $M^{T}$ belongs again to $\mathscr{H}_{\mathrm{loc}, 0}^{2}$. Let $M^{1}$ and $M^{2}$ be two local martingales in $\mathscr{Z}^{\mathscr{S}}$. By definition of $\mathscr{Z}^{\mathscr{S}}$, there exist two local martingales $M^{\left(\alpha_{1}\right)}$ and $M^{\left(\alpha_{2}\right)}$ in $\mathscr{Z}$ and two stopping times $T$ and $S$ in $\mathscr{S}$ such that $M^{1}=M^{\left(\alpha_{1}\right)^{T}}$ and $M^{2}=M^{\left(\alpha_{2}\right)^{S}}$. Then we have,

$$
\begin{aligned}
{\left[M^{1}, M^{2}\right]-\left\langle M^{1}, M^{2}\right\rangle } & =\left[M^{\left(\alpha_{1}\right)^{T}}, M^{\left(\alpha_{2}\right)^{S}}\right]-\left\langle M^{\left(\alpha_{1}\right)^{T}}, M^{\left(\alpha_{2}\right)^{S}}\right\rangle \\
& =\left[M^{\left(\alpha_{1}\right)}, M^{\left(\alpha_{2}\right)}\right]^{T \wedge S}-\left\langle M^{\left(\alpha_{1}\right)}, M^{\left(\alpha_{2}\right)}\right\rangle^{T \wedge S} \\
& =\left(\left[M^{\left(\alpha_{1}\right)}, M^{\left(\alpha_{2}\right)}\right]-\left\langle M^{\left(\alpha_{1}\right)}, M^{\left(\alpha_{2}\right)}\right\rangle\right)^{T \wedge S} \\
& =M^{\left(\alpha_{1}, \alpha_{2}\right)^{T \wedge S}} .
\end{aligned}
$$

By assumption, $T \wedge S$ belongs to $\mathscr{S}$ and $M^{\left(\alpha_{1}, \alpha_{2}\right)}$ belongs to $\mathscr{Z}$, because $\mathscr{Z}$ is a compensated-covariation stable family. Hence $\left[M^{1}, M^{2}\right]-\left\langle M^{1}, M^{2}\right\rangle \in \mathscr{Z}^{\mathscr{S}}$, meaning that $\mathscr{Z}^{\mathscr{S}}$ is again a compensated-covariation stable family of $\mathscr{H}_{\mathrm{loc}, 0}^{2}$.

For a compensated-covariation stable family in $\mathscr{H}_{\mathrm{loc}, 0}^{2}$ we can obtain a nice representation formula for products of its elements. The formula that we are going to show is very important for the further development. Before we need some technical preparation. The first lemma that we state concerns integration of measurable processes with respect to processes of finite variation.

4.1.3 Lemma. On the probability space $(\Omega, \mathscr{F}, \mathbb{P})$ we consider two measurable processes $H$ and $K$ and a process $A$ belonging to $\mathscr{V}$. If the integral processes $K \cdot A$ and $H \cdot(K \cdot A)$ belong to $\mathscr{V}$, the same holds for the integral process $(H K) \cdot A$ and the following formula holds:

$$
H \cdot(K \cdot A)=(H K) \cdot A \text {. }
$$

Proof. The proof of this result is classical and we sketch it very shortly. We first assume that $K \geq 0$ and $A \in \mathscr{V}^{+}$. In this special case, if we choose $H$ of the form $H:=1_{[0, s] \times B}$, where $s \geq 0$ and $B \in \mathscr{F}$, formula (4.5) is clearly true. The system of all such $H$ is stable under intersection. A monotone-class argument implies that (4.5) holds for every bounded $H$. If now $H$ is an arbitrary nonnegative process, approximating it by $H^{n}:=H \wedge n$, we can easily deduce (4.5) from the previous step. We now consider the general case of $A \in \mathscr{V}$ and $H$ and $K$ arbitrary. We know that there exist two unique processes $B, C$ in $\mathscr{V}^{+}$such that $A=B-C$ and $\operatorname{Var}(A)=B+C$ (cf. Jacod \& Shiryaev (2000), Proposition I.3.3). By assumption, the process $H \cdot(K \cdot A)$ belongs to $\mathscr{V}$ and so $\operatorname{Var}(H \cdot(K \cdot A))$ is finite-valued. For every process $J$ such that $J \cdot A \in \mathscr{V}$, we have $\operatorname{Var}(J \cdot A)=|J| \cdot \operatorname{Var}(A)$. Using this, we get

$$
\operatorname{Var}(H \cdot(K \cdot A))=|H| \cdot(|K| \cdot \operatorname{Var}(A))=|H| \cdot(|K| \cdot B)+|H| \cdot(|K| \cdot C)
$$

and, from the previous step, we obtain $\operatorname{Var}(H \cdot(K \cdot A))=(|H||K|) \cdot \operatorname{Var}(A)$, meaning that $(H K) \cdot A$ belongs to $\mathscr{V}$. Decomposing the processes $H$ and $K$ in the positive and negative part, $A$ as $A=B-C$ and using the linearity of the integral and the previous step it is now a simple computation to obtain (4.5). 
We stress that in the previous lemma the processes $H$ and $K$ were only measurable and not predictable.

In Jacod \& Shiryaev (2000), Proposition I.4.49.(a) it is stated that if $X$ is a semimartingale and $A$ belongs to $\mathscr{V}$, then the covariation between $X$ and $A$ can be represented as an integral with respect to $A$ as it follows:

$$
[X, A]=\Delta X \cdot A
$$

A consequence of (4.6) is that if $X$ is a semimartingale and $A$ belongs to $\mathscr{V}$, then the process $\Delta X \cdot A$ belongs to $\mathscr{V}$. From (4.6) and Lemma 4.1.3 we deduce the following result.

4.1.4 Lemma. Let $X^{1}, \ldots, X^{m}$ be semimartingales, $m \geq 1$ and $A \in \mathscr{V}$. The integral process $\left(\prod_{i=1}^{m} \Delta X^{i}\right) \cdot A$ belongs to $\mathscr{V}$.

Proof. We show this lemma by induction on $m$. If $m=1$, from (4.6), the result is true. Let us now assume the claim for $m$ and prove it for $m+1$. By the induction hypothesis the process $\left(\prod_{i=1}^{m} \Delta X^{i}\right) \cdot A$ belongs to $\mathscr{V}$ and, by (4.6), we have

$$
\left[X^{m+1},\left(\prod_{i=1}^{m} \Delta X^{i}\right) \cdot A\right]=\Delta X^{m+1} \cdot\left(\left(\prod_{i=1}^{m} \Delta X^{i}\right) \cdot A\right) .
$$

Hence the process on the right-hand side of the previous identity belongs to $\mathscr{V}$. From Lemma 4.5 , we have $\Delta X^{m+1} \cdot\left(\left(\prod_{i=1}^{m} \Delta X^{i}\right) \cdot A\right)=\left(\prod_{i=1}^{m+1} \Delta X^{i}\right) \cdot A$ and the proof is finished.

Now the necessary preparation is completed and we come, as announced, to the representation of products of local martingales belonging to a compensated-covariation stable family.

4.1.5 Proposition. Let $\mathscr{Z}:=\left\{M^{(\alpha)}, \alpha \in I\right\}$ be a compensated-covariation stable family of local martingales contained in $\mathscr{H}_{\mathrm{loc}, 0}^{2}$. For every $m \in \mathbb{N}$ and for every $\alpha_{1}, \ldots, \alpha_{m} \in I$, we have

$$
\begin{aligned}
& \prod_{i=1}^{m} M^{\left(\alpha_{i}\right)}=\sum_{i=1}^{m} \sum_{1 \leq j_{1}<\ldots<j_{i} \leq m}\left(\prod_{\substack{k=1 \\
k \neq j_{1}, \ldots, j_{i}}}^{m} M_{-}^{\left(\alpha_{k}\right)}\right) \cdot M^{\left(\alpha_{j_{1}}, \ldots, \alpha_{j_{i}}\right)} \\
& +\sum_{p=0}^{m-2} \sum_{i=p+2}^{m} \sum_{1 \leq j_{1}<\ldots<j_{i} \leq m}\left\{\left(\prod_{\substack{k=1 \\
k \neq j_{1}, \ldots, j_{i}}}^{m} M_{-}^{\left(\alpha_{k}\right)}\right) \times\right. \\
& \left.\times\left(\prod_{\ell=j_{i-p+1}}^{j_{i}} \Delta M^{\left(\alpha_{\ell}\right)}\right)\right\} \cdot\left\langle M^{\left(\alpha_{j_{1}}, \ldots, \alpha_{j_{i-p-1}}\right)}, M^{\left(\alpha_{j_{i-p}}\right)}\right\rangle .
\end{aligned}
$$

Proof. We show (4.7) by induction as a consequence of the formula of integration by parts (cf. (1.35)). We stress that $M_{-}^{(\alpha)}, \alpha \in I$, is locally bounded because $M_{0}^{(\alpha)}=0$. Hence the each summand in the first summation on the right-hand side of (4.7) is a local martingale. Lemma 4.1.4 ensures that every summand in the second summation appearing on the right-hand side of (4.7) is a process of finite variation. 
Therefore the right-hand side of (4.7) is well defined and is a semimartingale. By convention, $\prod_{\emptyset}=1$. After these preliminary remarks, we now start with the inductive argument. If $m=1$ there is nothing to prove. To begin with, for an easier understanding, we verify (4.7) for $m=2$ and we discuss it briefly for $m=3$. The formula of integration by parts implies that

$$
\begin{aligned}
M^{\left(\alpha_{1}\right)} M^{\left(\alpha_{2}\right)} & =M_{-}^{\left(\alpha_{1}\right)} \cdot M^{\left(\alpha_{2}\right)}+M_{-}^{\left(\alpha_{2}\right)} \cdot M^{\left(\alpha_{1}\right)}+\left[M^{\left(\alpha_{1}\right)}, M^{\left(\alpha_{2}\right)}\right] \\
& =M_{-}^{\left(\alpha_{1}\right)} \cdot M^{\left(\alpha_{2}\right)}+M_{-}^{\left(\alpha_{2}\right)} \cdot M^{\left(\alpha_{1}\right)}+M^{\left(\alpha_{1}, \alpha_{2}\right)}+\left\langle M^{\left(\alpha_{1}\right)}, M^{\left(\alpha_{2}\right)}\right\rangle,
\end{aligned}
$$

where $M^{(\alpha, \beta)}$ is the compensated-covariation process associated with $M^{(\alpha)}$ and $M^{(\beta)}$ (cf. (4.2)). Hence (4.7) holds for $m=2$. Notice that if $m=3$, by the formula of integration by parts, we get

$$
\begin{aligned}
& M^{\left(\alpha_{1}\right)} M^{\left(\alpha_{2}\right)} M^{\left(\alpha_{3}\right)} \\
& \quad=M_{-}^{\left(\alpha_{3}\right)} \cdot\left(M^{\left(\alpha_{1}\right)} M^{\left(\alpha_{2}\right)}\right)+\left(M_{-}^{\left(\alpha_{1}\right)} M_{-}^{\left(\alpha_{2}\right)}\right) \cdot M^{\left(\alpha_{3}\right)}+\left[M^{\left(\alpha_{1}\right)} M^{\left(\alpha_{2}\right)}, M^{\left(\alpha_{3}\right)}\right] .
\end{aligned}
$$

If we look back to the formula for $m=2$, it is clear that in the computation of $\left[M^{\left(\alpha_{1}\right)} M^{\left(\alpha_{2}\right)}, M^{\left(\alpha_{3}\right)}\right]$, the term $\left[\left\langle M^{\left(\alpha_{1}\right)}, M^{\left(\alpha_{2}\right)}\right\rangle, M^{\left(\alpha_{3}\right)}\right]$ appears. The process $M^{\left(\alpha_{3}\right)}$ is a local martingale while $\left\langle M^{\left(\alpha_{1}\right)}, M^{\left(\alpha_{2}\right)}\right\rangle$ is of finite variation. Therefore, from (4.6), we obtain: $\left[\left\langle M^{\left(\alpha_{1}\right)}, M^{\left(\alpha_{2}\right)}\right\rangle, M^{\left(\alpha_{3}\right)}\right]=\Delta M^{\left(\alpha_{3}\right)} \cdot\left\langle M^{\left(\alpha_{1}\right)}, M^{\left(\alpha_{2}\right)}\right\rangle$. In other words, the factor involving the jumps of the martingales $M^{(\alpha)}$ appearing in the second term on the right-hand side of (4.7) for $p>0$, comes into play only for $m \geq 3$. We now assume (4.7) for an arbitrary $m$ and we show it for $m+1$. From the formula of integration by parts, it follows

$$
\begin{aligned}
& \prod_{i=1}^{m+1} M^{\left(\alpha_{i}\right)}=M^{\left(\alpha_{m+1}\right)} \prod_{i=1}^{m} M^{\left(\alpha_{i}\right)} \\
& \quad=\left(\prod_{i=1}^{m} M_{-}^{\left(\alpha_{i}\right)}\right) \cdot M^{\left(\alpha_{m+1}\right)}+M_{-}^{\left(\alpha_{m+1}\right)} \cdot \prod_{i=1}^{m} M^{\left(\alpha_{i}\right)}+\left[\prod_{i=1}^{m} M^{\left(\alpha_{i}\right)}, M^{\left(\alpha_{m+1}\right)}\right] .
\end{aligned}
$$

By the induction hypothesis, we can compute explicitly the second and the third term in the last equality of (4.8). Indeed, for the second summand, by the induction hypothesis and Proposition 1.3.6, recalling that Lemma 4.1.4 ensures that every summand in the second summation appearing on the right-hand side of (4.7) is a process of finite variation, from (4.5), we get

$$
\begin{aligned}
& M_{-}^{\left(\alpha_{m+1}\right)} \cdot \prod_{i=1}^{m} M^{\left(\alpha_{i}\right)}=\sum_{i=1}^{m} \sum_{1 \leq j_{1}<\ldots<j_{i} \leq m}\left(M_{-}^{\left(\alpha_{m+1}\right)} \prod_{\substack{k=1 \\
k \neq j_{1}, \ldots, j_{i}}}^{m} M_{-}^{\left(\alpha_{k}\right)}\right) \cdot M^{\left(\alpha_{j_{1}}, \ldots, \alpha_{j_{i}}\right)} \\
&+\sum_{p=0}^{m-2} \sum_{i=p+2}^{m} \sum_{\substack{1 \leq j_{1}<\ldots<j_{i} \leq m \\
1}}^{m}\left\{\left(M_{-}^{\left(\alpha_{m+1}\right)} \prod_{\substack{k=1 \\
k \neq j_{1}, \ldots, j_{i}}}^{m} M_{-}^{\left(\alpha_{k}\right)}\right) \times\right. \\
&\left.\times\left(\prod_{\ell=j_{i-p+1}}^{j_{i}} \Delta M^{\left(\alpha_{\ell}\right)}\right)\right\} \cdot\left\langle M^{\left(\alpha_{\left.j_{1}, \ldots, \alpha_{j_{i-p-1}}\right)}\right)}, M^{\left(\alpha_{j_{i-p}}\right)}\right\rangle .
\end{aligned}
$$


Now we discuss the third summand in the last of the equalities in (4.8). We fix $1 \leq i \leq m$ and then $1 \leq j_{1}<\ldots<j_{i} \leq m$. Each one of the integrals $\left(\prod_{k \neq j_{1}, \ldots, j_{i}}^{m} M_{-}^{\left(\alpha_{k}\right)}\right)\left(\prod_{\ell=j_{i-p+1}}^{j_{i}} \Delta M^{\left(\alpha_{\ell}\right)}\right) \cdot\left\langle M^{\left(\alpha_{j_{1}}, \ldots, \alpha_{j_{i-p-1}}\right.}, M^{\left(\alpha_{j_{i-p}}\right)}\right\rangle$ belongs to $\mathscr{V}$. By the induction hypothesis and Proposition 1.3.4.(i), we have

$$
\begin{gathered}
{\left[\prod_{i=1}^{m} M^{\left(\alpha_{i}\right)}, M^{\left(\alpha_{j_{m+1}}\right)}\right]=\sum_{i=1}^{m} \sum_{1 \leq j_{1}<\ldots<j_{i} \leq m}\left(\prod_{\substack{k=1 \\
k \neq j_{1}, \ldots, j_{i}}}^{m} M_{-}^{\left(\alpha_{k}\right)}\right) \cdot\left[M^{\left(\alpha_{j_{1}}, \ldots, \alpha_{j_{i}}\right)}, M^{\left(\alpha_{m+1}\right)}\right]} \\
+\sum_{p=0}^{m-2} \sum_{i=p+2}^{m} \sum_{\substack{1 \leq j_{1}<\ldots<j_{i} \leq m\\
}}\left\{\left[M^{\left(\alpha_{m+1}\right)},\left(\prod_{\substack{k=1 \\
k \neq j_{1}, \ldots, j_{i}}}^{m} M_{-}^{\left(\alpha_{k}\right)}\right) \times\right.\right. \\
\left.\left.\times\left(\prod_{\ell=j_{i-p+1}}^{j_{i}} \Delta M^{\left(\alpha_{\ell}\right)}\right)\right\} \cdot\left\langle M^{\left(\alpha_{j_{1}}, \ldots, \alpha_{j_{i-p-1}}\right)}, M^{\left(\alpha_{j_{i-p}}\right)}\right\rangle\right] .
\end{gathered}
$$

From (4.6) and Lemma 4.1.3 we can conclude that

$$
\begin{aligned}
& {\left[\prod_{i=1}^{m} M^{\left(\alpha_{i}\right)}, M^{\left(\alpha_{j_{m+1}}\right)}\right] }=\sum_{i=1}^{m} \sum_{1 \leq j_{1}<\ldots<j_{i} \leq m}\left(\prod_{\substack{k=1 \\
k \neq j_{1}, \ldots, j_{i}}}^{m} M_{-}^{\left(\alpha_{k}\right)}\right) \cdot\left[M^{\left(\alpha_{j_{1}}, \ldots, \alpha_{j_{i}}\right)}, M^{\left(\alpha_{m+1}\right)}\right] \\
&+\sum_{p=0}^{m-2} \sum_{i=p+2}^{m} \sum_{1 \leq j_{1}<\ldots<j_{i} \leq m}\left\{\left(\prod_{\substack{k=1 \\
k \neq j_{1}, \ldots, j_{i}}}^{m} M_{-}^{\left(\alpha_{k}\right)}\right) \times\right. \\
&\left.\times\left(\Delta M^{\left(\alpha_{m+1}\right)} \prod_{\ell=j_{i-p+1}}^{j_{i}} \Delta M^{\left(\alpha_{\ell}\right)}\right)\right\} \cdot\left\langle M^{\left(\alpha_{j_{1}}, \ldots, \alpha_{j_{i-p-1}}\right)}, M^{\left(\alpha_{j_{i-p}}\right)}\right\rangle .
\end{aligned}
$$

Using (4.3) in the first term on the right-hand side of the previous formula it follows:

$$
\begin{aligned}
& {\left[\prod_{i=1}^{m} M^{\left(\alpha_{i}\right)}, M^{\left(\alpha_{j_{m}+1}\right)}\right]=\sum_{i=1}^{m} \sum_{1 \leq j_{1}<\ldots<j_{i} \leq m}\left(\prod_{\substack{k=1 \\
k \neq j_{1}, \ldots, j_{i}}}^{m} M_{-}^{\left(\alpha_{k}\right)}\right) \cdot M^{\left(\alpha_{j_{1}}, \ldots, \alpha_{j_{i}}, \alpha_{m+1}\right)}} \\
& +\sum_{i=1}^{m} \sum_{1 \leq j_{1}<\ldots<j_{i} \leq m}\left(\prod_{\substack{k=1 \\
k \neq j_{1}, \ldots, j_{i}}}^{m} M_{-}^{\left(\alpha_{k}\right)}\right) \cdot\left\langle M^{\left(\alpha_{j_{1}}, \ldots, \alpha_{j_{i}}\right)}, M^{\left(\alpha_{m+1}\right)}\right\rangle \\
& +\sum_{p=0}^{m-2} \sum_{i=p+2}^{m} \sum_{1 \leq j_{1}<\ldots<j_{i} \leq m}\left\{\left(\prod_{\substack{k=1 \\
k \neq j_{1}, \ldots, j_{i}}}^{m} M_{-}^{\left(\alpha_{k}\right)}\right) \times\right. \\
& \left.\times\left(\Delta M^{\left(\alpha_{m+1}\right)} \prod_{\substack{j_{i} \\
\ell=j_{i-p+1}}}^{m} \Delta M^{\left(\alpha_{\ell}\right)}\right)\right\} \cdot\left\langle M^{\left(\alpha_{j_{1}}, \ldots, \alpha_{j_{i-p-1}}\right)}, M^{\left(\alpha_{j_{i-p}}\right)}\right\rangle .
\end{aligned}
$$


Inserting (4.9) and (4.10) in (4.8) we get:

$$
\begin{aligned}
& \prod_{i=1}^{m+1} M^{\left(\alpha_{i}\right)}=\left(\prod_{i=1}^{m} M_{-}^{\left(\alpha_{i}\right)}\right) \cdot M^{\left(\alpha_{m+1}\right)} \\
& +\sum_{i=1}^{m} \sum_{1 \leq j_{1}<\ldots<j_{i} \leq m}\left(M_{-}^{\left(\alpha_{m+1}\right)} \prod_{\substack{k=1 \\
k \neq j_{1}, \ldots, j_{i}}}^{m} M_{-}^{\left(\alpha_{k}\right)}\right) \cdot M^{\left(\alpha_{j_{1}}, \ldots, \alpha_{j_{i}}\right)} \\
& +\sum_{i=1}^{m} \sum_{1 \leq j_{1}<\ldots<j_{i} \leq m}\left(\prod_{\substack{k=1 \\
k \neq j_{1}, \ldots, j_{i}}}^{m} M_{-}^{\left(\alpha_{k}\right)}\right) \cdot M^{\left(\alpha_{j_{1}}, \ldots, \alpha_{j_{i}}, \alpha_{m+1}\right)} \\
& +\sum_{p=0}^{m-2} \sum_{i=p+2}^{m} \sum_{1 \leq j_{1}<\ldots<j_{i} \leq m}\left\{\left(M_{-}^{\left(\alpha_{m+1}\right)} \prod_{\substack{k=1 \\
k \neq j_{1}, \ldots, j_{i}}}^{m} M_{-}^{\left(\alpha_{k}\right)}\right) \times\right. \\
& \left.\times\left(\prod_{\ell=j_{i-p+1}}^{j_{i}} \Delta M^{\left(\alpha_{\ell}\right)}\right)\right\} \cdot\left\langle M^{\left(\alpha_{j_{1}}, \ldots, \alpha_{j_{i-p-1}}\right)}, M^{\left(\alpha_{j_{i-p}}\right)}\right\rangle \\
& +\sum_{i=1}^{m} \sum_{1 \leq j_{1}<\ldots<j_{i} \leq m}\left(\prod_{\substack{k=1 \\
k \neq j_{1}, \ldots, j_{i}}}^{m+1} M_{-}^{\left(\alpha_{k}\right)}\right) \cdot\left\langle M^{\left(\alpha_{j_{1}}, \ldots, \alpha_{j_{i}}\right)}, M^{\left(\alpha_{m+1}\right)}\right\rangle \\
& +\sum_{p=0}^{m-2} \sum_{i=p+2}^{m} \sum_{1 \leq j_{1}<\ldots<j_{i} \leq m}\left\{\left(\prod_{\substack{k=1 \\
k \neq j_{1}, \ldots, j_{i}}}^{m} M_{-}^{\left(\alpha_{k}\right)}\right) \times\right. \\
& \left.\times\left(\Delta M^{\left(\alpha_{m+1}\right)} \prod_{\ell=j_{i-p+1}}^{j_{i}} \Delta M^{\left(\alpha_{\ell}\right)}\right)\right\} \cdot\left\langle M^{\left(\alpha_{j_{1}}, \ldots, \alpha_{j_{i-p-1}}\right)}, M^{\left(\alpha_{j_{i-p}}\right)}\right\rangle .
\end{aligned}
$$

Let us now discuss $(4.11),(4.12)$ and (4.13). We can rewrite (4.13) as it follows

$$
(4.13)=\sum_{i=2}^{m+1} \sum_{\substack{1 \leq j_{1}<\ldots<j_{i} \leq m+1 \\ j_{i}=m+1}}\left(\prod_{\substack{k=1 \\ k \neq j_{1}, \ldots, j_{i}}}^{m+1} M_{-}^{\left(\alpha_{k}\right)}\right) \cdot M^{\left(\alpha_{j_{1}}, \ldots, \alpha_{j_{i}}\right)}
$$

while (4.11) is the term for $i=1$. Putting together (4.11) and (4.13), we obtain

$$
(4.11)+(4.13)=\sum_{i=1}^{m+1} \sum_{\substack{1 \leq j_{1}<\ldots<j_{i} \leq m+1 \\ j_{i}=m+1}}\left(\prod_{\substack{k=1 \\ k \neq j_{1}, \ldots, j_{i}}}^{m+1} M_{-}^{\left(\alpha_{k}\right)}\right) \cdot M^{\left(\alpha_{j_{1}}, \ldots, \alpha_{j_{i}}\right)} .
$$

Furthermore, (4.12) can be written as

$$
(4.12)=\sum_{i=1}^{m+1} \sum_{\substack{1 \leq j_{1}<\ldots<j_{i} \leq m+1 \\ j_{i} \neq m+1}}\left(\prod_{\substack{k=1 \\ k \neq j_{1}, \ldots, j_{i}}}^{m+1} M_{-}^{\left(\alpha_{k}\right)}\right) \cdot M^{\left(\alpha_{j_{1}}, \ldots, \alpha_{j_{i}}\right)}
$$

and this yields

$$
(4.11)+(4.12)+(4.13)=\sum_{i=1}^{m+1} \sum_{1 \leq j_{1}<\ldots<j_{i} \leq m+1}\left(\prod_{\substack{k=1 \\ k \neq j_{1}, \ldots, j_{i}}}^{m+1} M_{-}^{\left(\alpha_{k}\right)}\right) \cdot M^{\left(\alpha_{j_{1}}, \ldots, \alpha_{j_{i}}\right)}
$$


which is the first summand on the right-hand side of (4.7) for $m+1$. Now we come to the discussion of (4.14), (4.15) and (4.16). For (4.15) we have

$$
(4.15)=\sum_{i=2}^{m+1} \sum_{\substack{1 \leq j_{1}<\ldots<j_{i} \leq m+1 \\ j_{i}=m+1}}\left(\prod_{\substack{k=1 \\ k \neq j_{1}, \ldots, j_{i}}}^{m+1} M_{-}^{\left(\alpha_{k}\right)}\right) \cdot\left\langle M^{\left(\alpha_{j_{1}}, \ldots, \alpha_{j_{i-1}}\right)}, M^{\left(\alpha_{j_{i}}\right)}\right\rangle .
$$

Setting $p^{\prime}:=p+1$ and denoting it again by $p,(4.16)$ gives

$$
\begin{aligned}
(4.16)= & \sum_{p=1}^{(m+1)} \sum_{i=p+2}^{-2} \sum_{\substack{1 \leq j_{1}<\ldots<j_{i} \leq m+1 \\
j_{i}=m+1}}^{m+1}\left\{\left(\prod_{\substack{k=1 \\
k \neq j_{1}, \ldots, j_{i}}}^{m+1} M_{-}^{\left(\alpha_{k}\right)}\right) \times\right. \\
& \left.\times\left(\prod_{\ell=j_{i-p+1}}^{j_{i}} \Delta M^{\left(\alpha_{\ell}\right)}\right)\right\} \cdot\left\langle M^{\left(\alpha_{j_{1}}, \ldots, \alpha_{j_{i-p-1}}\right)}, M^{\left(\alpha_{j_{i-p}}\right)}\right\rangle .
\end{aligned}
$$

If we put together (4.15) and (4.16), we obtain

$$
\begin{aligned}
(4.15)+(4.16)= & \sum_{p=0}^{m-1} \sum_{i=p+2}^{m+1} \sum_{\substack{1 \leq j_{1}<\ldots<j_{i} \leq m+1 \\
j_{i}=m+1}}\left\{\left(\prod_{\substack{k=1 \\
k \neq j_{1}, \ldots, j_{i}}}^{m+1} M_{-}^{\left(\alpha_{k}\right)}\right) \times\right. \\
& \left.\times\left(\prod_{\ell=j_{i-p+1}}^{j_{i}} \Delta M^{\left(\alpha_{\ell}\right)}\right)\right\} \cdot\left\langle M^{\left(\alpha_{j_{1}}, \ldots, \alpha_{j_{i-p-1}}\right)}, M^{\left(\alpha_{j_{i-p}}\right)}\right\rangle .
\end{aligned}
$$

Finally, (4.14) can be written as

$$
\begin{aligned}
(4.14)= & \sum_{p=0}^{(m+1)} \sum_{i=p+2}^{-2} \sum_{\substack{1 \leq j_{1}<\ldots<j_{i} \leq m+1 \\
j_{i} \neq m+1}}^{m+1}\left\{\left(\prod_{\substack{k=1 \\
k \neq j_{1}, \ldots, j_{i}}}^{m+1} M_{-}^{\left(\alpha_{k}\right)}\right) \times\right. \\
& \left.\times\left(\prod_{\ell=j_{i-p+1}}^{j_{i}} \Delta M^{\left(\alpha_{\ell}\right)}\right)\right\} \cdot\left\langle M^{\left(\alpha_{j_{1}}, \ldots, \alpha_{j_{i-p-1}}\right)}, M^{\left(\alpha_{j_{i-p}}\right)}\right\rangle .
\end{aligned}
$$

Notice that in the previous formula, when $p=(m+1)-2$, we get that $\left\{j_{1}, \ldots, j_{i}\right\}$ is equal to the empty set. Indeed, this case for $p$ yields $i=m+1$ but, from $j_{i} \neq m+1$, the case $\left\{j_{1}, \ldots, j_{i}\right\}$ with $i=m+1$ is impossible. As a result, we get

$$
\begin{aligned}
(4.14)+(4.15)+(4.16)= & \sum_{p=0}^{m-1} \sum_{i=p+2}^{m+1} \sum_{1 \leq j_{1}<\ldots<j_{i} \leq m+1}\left\{\left(\prod_{\substack{k=1 \\
k \neq j_{1}, \ldots, j_{i}}}^{m+1} M_{-}^{\left(\alpha_{k}\right)}\right) \times\right. \\
& \left.\times\left(\prod_{\ell=j_{i-p+1}}^{j_{i}} \Delta M^{\left(\alpha_{\ell}\right)}\right)\right\} \cdot\left\langle M^{\left(\alpha_{j_{1}}, \ldots, \alpha_{j_{i-p-1}}\right)}, M^{\left(\alpha_{j_{i-p}}\right)}\right\rangle .
\end{aligned}
$$

This is the second summand on the right-hand side of (4.7) for $m+1$ and the proof is finished. 
We remark that if $\mathscr{Z}$ is a compensated-covariation stable family consisting of quasi-left continuous local martingales, then (4.7) becomes considerably simpler. Indeed, according with Jacod \& Shiryaev (2000), Theorem I.4.2, in this case we can choose a continuous version of the process $\left\langle M^{(\alpha)}, M^{(\beta)}\right\rangle, \alpha, \beta \in I$, and so all the terms appearing in the second summation on the right-hand side of (4.7) vanishes for $p \neq 0$.

Let $\mathscr{Z}:=\left\{M^{(\alpha)}, \alpha \in I\right\}$ be a family of local martingales in $\mathscr{H}_{\mathrm{loc}, 0}^{2}$ (not necessarily compensated-covariation stable). By $\mathscr{F}_{\infty}^{\mathscr{Z}}$ we denote the $\mathbb{P}$-completion of the $\sigma$ algebra $\sigma\left(M_{t}^{(\alpha)}, t \geq 0, \alpha \in I\right)$ in itself, i.e.,

$$
\mathscr{F}_{\infty}^{\mathscr{Z}}:=\sigma\left(M_{t}^{(\alpha)}, t \geq 0, \alpha \in I\right) \vee \mathscr{N}(\mathbb{P})
$$

where $\mathscr{N}(\mathbb{P})$ indicates the null-sets of $\mathbb{P}$ in $\sigma\left(M_{t}^{(\alpha)}, t \geq 0, \alpha \in I\right)$. The filtration $\mathbb{F}_{+}^{\mathscr{Z}}:=\left(\mathscr{F}_{t+}^{\mathscr{Z}}\right)_{t \geq 0}$ is the natural filtration of $\mathscr{Z}$, that is, the smallest filtration satisfying the usual conditions and with respect to which each process in $\mathscr{Z}$ is an adapted local martingale, thus,

$$
\mathscr{F}_{t+}^{\mathscr{Z}}:=\bigcap_{\varepsilon>0} \sigma\left(M_{r}^{(\alpha)}, \quad 0 \leq r \leq t+\varepsilon, \quad \alpha \in I\right) \vee \mathscr{N}(\mathbb{P}), \quad t \geq 0 .
$$

From now on, we restrict our attention to the probability space $\left(\Omega, \mathscr{F}_{\infty}^{\mathscr{Z}}, \mathbb{P}\right)$ and to the filtration $\mathbb{F}_{+}^{\mathscr{Z}}$, i.e., $\mathscr{Z}$ is a family of compensated-covariation stable martingales contained in $\mathscr{H}_{\mathrm{loc}, 0}^{2}\left(\mathbb{F}_{+}^{\mathscr{Z}}\right)$ on the probability space $\left(\Omega, \mathscr{F}_{\infty}^{\mathscr{Z}}, \mathbb{P}\right)$.

In the remaining of this section, we look for sufficient conditions to ensure that $\mathscr{Z}$ possesses the $\mathscr{H}^{2}$-PRP with respect to $\mathbb{F}_{+}^{\mathscr{Z}}$. The first assumption which we make concerns the existence of moments for the elements of $\mathscr{Z}$. We assume that $\mathscr{Z} \subseteq \mathscr{H}_{\mathrm{loc}, 0}^{2}$ is such that for every $M^{(\alpha)}, \alpha \in I$, and $t \geq 0$ there exists a $c_{\alpha}(t)>0$ such that

$$
\mathbb{E}\left[\exp \left(c_{\alpha}(t)\left|M_{t}^{(\alpha)}\right|\right)\right]<+\infty, \quad t \geq 0 .
$$

In other words, we require that each element of $\mathscr{Z}$ is such that its state at time $t \geq 0$ admits at least one exponential moment, for every $t \geq 0$. If $\mathscr{Z}$ is a family of true martingales contained in $\mathscr{H}_{0}^{2}$ we can replace (4.17) by the following assumption: For every $M^{(\alpha)}, \alpha \in I$, there exists a $c_{\alpha}>0$ such that

$$
\mathbb{E}\left[\exp \left(c_{\alpha}\left|M_{\infty}^{(\alpha)}\right|\right)\right]<+\infty
$$

Notice that (4.18) is stronger than (4.17). Indeed, if $M^{(\alpha)}$ is a martingale, from Jensen's inequality, the process $\exp \left(c_{\alpha}\left|M^{(\alpha)}\right|\right)$ is a submartingale. Hence the function $t \mapsto \mathbb{E}\left[\left(c_{\alpha}\left|M_{t}^{(\alpha)}\right|\right)\right]$ is increasing and so

$$
\mathbb{E}\left[\exp \left(c_{\alpha}\left|M_{t}^{(\alpha)}\right|\right)\right] \leq \mathbb{E}\left[\exp \left(c_{\alpha}\left|M_{\infty}^{(\alpha)}\right|\right)\right]<+\infty .
$$

In the latter case the constant $c_{\alpha}>0$ is universal and not depending on time. If $\mathscr{Z} \subseteq \mathscr{H}_{\mathrm{loc}, 0}^{2}$ satisfies (4.17), then each random variable $M_{t}^{(\alpha)}, t \geq 0$, has a finite moment of every order. This is an immediate consequence of the Taylor expansion of the exponential function. If $\mathscr{Z} \subseteq \mathscr{H}_{0}^{2}$ satisfies (4.18), the random variable $M_{\infty}^{(\alpha)}$ 
has finite moment of every order and hence the same holds for $M_{t}^{(\alpha)}, t \geq 0$, for every $\alpha \in I$. We now recall the very well-known estimation of the geometric mean by the arithmetic mean, which will be often used in the sequel. For every $a_{1}, \ldots, a_{m} \geq 0$, $m \in \mathbb{N}$, it follows:

$$
\left(\prod_{i=1}^{m} a_{i}\right)^{\frac{1}{m}} \leq \frac{1}{m} \sum_{i=1}^{m} a_{i}
$$

For our purpose, the most important consequences of (4.17) are the following. We define the family $\mathscr{K}$ by

$$
\mathscr{K}:=\left\{\prod_{i=1}^{m} M_{t_{i}}^{\left(\alpha_{i}\right)}, \quad \alpha_{i} \in I, \quad i=1, \ldots, m, \quad t_{i} \in \mathbb{R}, \quad m \in \mathbb{N}\right\}
$$

which is the family of monomials generated by products of elements of $\mathscr{Z}$ at different times. If $\mathscr{Z} \subseteq \mathscr{H}_{\text {loc }, 0}^{2}$ satisfies (4.17), then $\mathscr{K} \subseteq L^{2}\left(\Omega, \mathscr{F}_{\infty}^{\mathscr{Z}}, \mathbb{P}\right)$. Indeed, by (4.19), for every integer $p \geq 1, \alpha_{1}, \ldots, \alpha_{m} \in I$ and $t_{1}, \ldots, t_{m} \in \mathbb{R}_{+}, m \in \mathbb{N}$, we have

$$
\mathbb{E}\left[\prod_{i=1}^{m}\left|M_{t_{i}}^{\left(\alpha_{i}\right)}\right|^{p}\right]=\mathbb{E}\left[\left(\prod_{i=1}^{m}\left|M_{t_{i}}^{\left(\alpha_{i}\right)}\right|^{p m}\right)^{\frac{1}{m}}\right] \leq \sum_{i=1}^{m} \mathbb{E}\left[\left|M_{t_{i}}^{\left(\alpha_{i}\right)}\right|^{p m}\right]<+\infty
$$

where, in the last estimation, we used that (4.17) implies that $M_{t_{i}}^{\left(\alpha_{i}\right)}$ has a finite moment of every order, $\alpha_{i} \in I, t_{i} \in \mathbb{R}_{+}, i=1, \ldots, m$. In particular, for $p=2$, we obtain the assertion. Furthermore, the following proposition holds.

4.1.6 Proposition. Let $\mathscr{Z} \subseteq \mathscr{H}_{\mathrm{loc}, 0}^{2}$ satisfy (4.17). The family $\mathscr{K}$ defined in (4.20) is total in $L^{2}\left(\Omega, \mathscr{F}_{\infty}^{\mathscr{Z}}, \mathbb{P}\right)$.

Proof. cf. Theorem B.5.

Before to come to the discussion of the $\mathscr{H}^{2}$-PRP, we need some technical preparation. We begin with the following lemma.

4.1.7 Lemma. Let $A \in \mathscr{A}$ be a deterministic process and $\mathscr{Z}:=\left\{M^{(\alpha)}, \alpha \in I\right\}$ be a family of martingales contained in $\mathscr{H}_{0}^{2}$ satisfying (4.18). Let $m, p, q \in \mathbb{N}, q \leq m$. For $\alpha_{i} \in I, i=1, \ldots, m$, we define the processes $H$ and $K$ by

$$
H:=\prod_{i=1}^{m}\left|M_{-}^{\left(\alpha_{i}\right)}\right|^{p}, \quad K:=\left(\prod_{i=1}^{q} \Delta M_{t}^{\left(\alpha_{j_{i}}\right)} \prod_{\substack{k=1 \\ k \neq j_{1}, \ldots, j_{q}}}^{m} M_{t-}^{\left(\alpha_{k}\right)}\right) .
$$

The processes $H \cdot A$ and $K \cdot A$ belong to $\mathscr{A}$.

Proof. Notice that both the processes $H \cdot A$ and $K \cdot A$ are well defined and belong to $\mathscr{V}$. Indeed, $H \cdot A \in \mathscr{V}$ because $H$ is a locally bounded process. For $K \cdot A$ the statement is easily deduced from Lemma 4.1.4. The proof that $K \cdot A$ is of integrable variation is similar to the one that $H \cdot A$ is of integrable variation. We verify the statement in a detailed way only for $H \cdot A$. In a first step we assume that $A \in \mathscr{A}^{+}$, i.e., that $A$ is an integrable increasing process. Because $A$ is, by assumption, deterministic, to require $A \in \mathscr{A}^{+}$is equivalent to require that $A_{\infty}$ is finite valued. We have to show that $H \cdot A_{\infty}$ belongs to $L^{1}(\mathbb{P})$. The measure $\mu^{A}(\cdot)$ defined on $\left(\mathbb{R}_{+}, \mathscr{B}(\mathbb{R})\right)$ by

$$
\mu^{A}([0, t]):=A_{t}, \quad t \geq 0,
$$


is a finite measure. Indeed, $\mu^{A}([0,+\infty])=A_{\infty}<+\infty$. Therefore the measure $\mathbb{P} \otimes \mu^{A}$ is a finite measure on $\left(\Omega \times \mathbb{R}_{+}, \mathscr{F} \otimes \mathscr{B}\left(\mathbb{R}_{+}\right)\right)$. For this reason, as a consequence of the theorem of Fubini (cf. Bauer (2001), Corollary III.23.7), we can write

$$
\mathbb{E}\left[H \cdot A_{\infty}\right]=\mathbb{E}[H] \cdot A_{\infty},
$$

where by $\mathbb{E}[H]$ we denote the function $t \mapsto \mathbb{E}\left[H_{t}\right], t \geq 0$. By the definition of $H$ and the estimate (4.19), from (4.22) we obtain:

$$
\begin{aligned}
\mathbb{E}\left[H \cdot A_{\infty}\right]=\mathbb{E}[H] \cdot A_{\infty} & :=\int_{0}^{+\infty} \mathbb{E}\left[\prod_{i=1}^{m}\left|M_{t-}^{\left(\alpha_{i}\right)}\right|^{p}\right] \mathrm{d} A_{t} \\
& =\int_{0}^{+\infty} \mathbb{E}\left[\left(\prod_{i=1}^{m}\left|M_{t-}^{\left(\alpha_{i}\right)}\right|^{p m}\right)^{\frac{1}{m}}\right] \mathrm{d} A_{t} \\
& \leq \int_{0}^{+\infty} \mathbb{E}\left[\sum_{i=1}^{m}\left|M_{t-}^{\left(\alpha_{i}\right)}\right|^{p m}\right] \mathrm{d} A_{t} .
\end{aligned}
$$

By assumption, the process $M^{\left(\alpha_{i}\right)}$ belongs to $\mathscr{H}_{0}^{2}$, for every $i=1, \ldots, m$ and, thanks to (4.18), $\left|M_{\infty}^{\left(\alpha_{i}\right)}\right|^{p m} \in L^{1}(\mathbb{P})$. From Dellacherie (1972), Theorem V.10, it follows that $M_{t-}^{\left(\alpha_{i}\right)}=\mathbb{E}\left[M_{\infty}^{\left(\alpha_{i}\right)} \mid \mathscr{F}_{t-}\right]$ and so, for every $m \in \mathbb{N}$, we have

$$
\mathbb{E}\left[\left|M_{t-}^{\left(\alpha_{i}\right)}\right|^{p m}\right] \leq \mathbb{E}\left[\left|M_{\infty}^{\left(\alpha_{i}\right)}\right|^{p m}\right]<+\infty .
$$

Therefore

$$
\begin{aligned}
\mathbb{E}\left[H \cdot A_{\infty}\right] \leq \int_{0}^{+\infty} \mathbb{E}\left[\sum_{i=1}^{m}\left|M_{t-}^{\left(\alpha_{i}\right)}\right|^{p m}\right] \mathrm{d} A_{t} & =\int_{0}^{+\infty} \sum_{i=1}^{m} \mathbb{E}\left[\left|M_{t-}^{\left(\alpha_{i}\right)}\right|^{p m}\right] \mathrm{d} A_{t} \\
& \leq \int_{0}^{+\infty} \sum_{i=1}^{m} \mathbb{E}\left[\left|M_{\infty}^{\left(\alpha_{i}\right)}\right|^{p m}\right] \mathrm{d} A_{t} \\
& =\sum_{i=1}^{m} \mathbb{E}\left[\left|M_{\infty}^{\left(\alpha_{i}\right)}\right|^{p m}\right] A_{\infty}<\infty
\end{aligned}
$$

and this concludes the proof of the lemma for $A \in \mathscr{A}^{+}$. Let us now consider the general case in which $A$ is a deterministic process belonging to $\mathscr{A}$, i.e., a deterministic process of finite variation such that $A_{\infty}<+\infty$. By Jacod \& Shiryaev (2000), Proposition I.3.3, there exist two unique increasing processes $B, C \in \mathscr{A}^{+}$such that $A=B-C$ and $\operatorname{Var}(A)=B+C$. We show that the random variable $\operatorname{Var}(H \cdot A)_{\infty}$ belongs to $L^{1}(\mathbb{P})$. The process $H \geq 0$ is locally bounded and this ensures that $H \cdot A \in \mathscr{V}$. Furthermore, the relation $\operatorname{Var}(H \cdot A)=H \cdot \operatorname{Var}(A)$ implies

$$
\operatorname{Var}(H \cdot A)_{\infty}=H \cdot \operatorname{Var}(A)_{\infty}=H \cdot B_{\infty}+H \cdot C_{\infty}
$$

and thanks to the previous step we can conclude. If $A \in \mathscr{A}^{+}$the prove that $K \cdot A \in \mathscr{A}$ is similar to the one for $H$. In the general case of $A \in \mathscr{A}$, we have to apply Lemma 4.1.3 and Lemma 4.1.4. 
Now we can immediately deuce that if $\mathscr{Z} \subseteq \mathscr{H}_{0}^{2}$ satisfies $(4.18)$ and $M^{(\alpha)} \in \mathscr{Z}$ is such that $\left\langle M^{(\alpha)}, M^{(\alpha)}\right\rangle$ is deterministic, then the predictable process $\prod_{i=1}^{m} M_{-}^{\left(\alpha_{i}\right)}$ belongs to $\mathrm{L}^{2}\left(M^{(\alpha)}\right)$ (cf. (1.26)), for every $\alpha_{i} \in I, i=1, \ldots, m$. Indeed, because $M^{(\alpha)} \in \mathscr{H}_{0}^{2}$, the process $\left[M^{(\alpha)}, M^{(\alpha)}\right]$ belongs to $\mathscr{A}^{+}$and $\left\langle M^{(\alpha)}, M^{(\alpha)}\right\rangle$ is its compensator (cf. Proposition 1.2.11.(ii)). Hence

$$
\mathbb{E}\left[\left(\prod_{i=1}^{m} M_{-}^{\left(\alpha_{i}\right)^{2}}\right) \cdot\left[M^{(\alpha)}, M^{(\alpha)}\right]_{\infty}\right]=\mathbb{E}\left[\left(\prod_{i=1}^{m} M_{-}^{\left(\alpha_{i}\right)^{2}}\right) \cdot\left\langle M^{(\alpha)}, M^{(\alpha)}\right\rangle_{\infty}\right] .
$$

From Lemma 4.1.7 it follows that the right-hand side in the previous relation is integrable and the assertion is proved. We are going to discuss a lemma which concerns the orthogonality of a random variable $N \in L^{2}(\mathbb{P})$ to products of elements of $\mathscr{K}$.

4.1.8 Lemma. Let $\mathscr{Z} \subseteq \mathscr{H}_{0}^{2}$ be a family of martingales which satisfies (4.18), $m \in \mathbb{N}$ and $N \in L^{2}(\mathbb{P})$ such that for every $\alpha_{1}, \ldots, \alpha_{m} \in I$, for every $t_{1}, \ldots, t_{m} \in \mathbb{R}_{+}$, $N$ is orthogonal in $L^{2}(\mathbb{P})$ to $\prod_{i=1}^{m} M_{t_{i}}^{\left(\alpha_{i}\right)}$. Then we also have

$$
N \perp\left(\prod_{i=1}^{q} M_{t}^{\left(\alpha_{j_{i}}\right)} \prod_{\substack{k=1 \\ k \neq j_{1}, \ldots, j_{q}}}^{m} M_{t-}^{\left(\alpha_{k}\right)}\right), \quad q \leq m, \quad 1 \leq j_{1}<\ldots<j_{q} \leq m .
$$

Proof. We take $q \leq m$ and $1 \leq j_{1}<\ldots<j_{q} \leq m$. Let $\left(t_{n}\right)_{n \geq 1} \subseteq \mathbb{R}_{+}$be such that $t_{n} \uparrow t$. Then we have

$$
\prod_{\substack{k=1 \\ k \neq j_{1}, \ldots, j_{q}}}^{m} M_{t-}^{\left(\alpha_{k}\right)}=\lim _{n \rightarrow+\infty} \prod_{\substack{k=1 \\ k \neq j_{1}, \ldots, j_{q}}}^{m} M_{t_{n}}^{\left(\alpha_{k}\right)}, \quad \text { a.s. }
$$

Let $\beta_{1}, \ldots, \beta_{m} \in I$ be such that $M^{\left(\beta_{j_{i}}\right)}:=M^{\left(\alpha_{j_{i}}\right)}$, if $i=1, \ldots, q$ and $M^{\left(\beta_{k}\right)}:=M^{\left(\alpha_{k}\right)}$, if $k \neq j_{1}, \ldots, j_{q}$. Analogously, we introduce the sequence $\left(s_{i}\right)_{i \geq 1}$ of deterministic times as $s_{j_{i}}=t$, if $i=1, \ldots, q$ and $s_{k}=t_{n}$ if $k \neq j_{1}, \ldots, j_{q}$. Then, for every fixed $n \geq 1$, we have

$$
\prod_{i=1}^{q} M_{t}^{\left(\alpha_{j_{i}}\right)} \prod_{\substack{k=1 \\ k \neq j_{1}, \ldots, j_{q}}}^{m} M_{t_{n}}^{\left(\alpha_{k}\right)}=\prod_{i=1}^{m} M_{s_{i}}^{\left(\beta_{i}\right)}
$$

meaning that for every fixed $n \geq 1$ it follows:

$$
\mathbb{E}\left[N \prod_{i=1}^{q} M_{t}^{\left(\alpha_{j_{i}}\right)} \prod_{\substack{k=1 \\ k \neq j_{1}, \ldots, j_{q}}}^{m} M_{t_{n}}^{\left(\alpha_{k}\right)}\right]=0 .
$$

We now show that the sequence $\left(a_{n}\right)_{n \geq 1}$ defined by

$$
a_{n}:=N\left(\prod_{i=1}^{q} M_{t}^{\left(\alpha_{j_{i}}\right)} \prod_{\substack{k=1 \\ k \neq j_{1}, \ldots, j_{q}}}^{m} M_{t_{n}}^{\left(\alpha_{k}\right)}\right), \quad n \geq 1
$$


is dominated in $L^{1}(\mathbb{P})$. By (4.19) we have

$$
\left|a_{n}\right| \leq N^{2}+\prod_{i=1}^{q} M_{t}^{\left(\alpha_{j_{i}}\right)^{2}} \prod_{\substack{k=1 \\ k \neq j_{1}, \ldots, j_{q}}}^{m} M_{t_{n}}^{\left(\alpha_{k}\right)^{2}} .
$$

By assumption $N \in L^{2}(\mathbb{P})$ and so it remains to show that the second summand on the right-hand side of the previous inequality is integrable. Again by (4.19), for every $n \geq 1$, we obtain

$$
\begin{aligned}
\prod_{i=1}^{q} M_{t}^{\left(\alpha_{j_{i}}\right)^{2}} \prod_{\substack{k=1 \\
k \neq j_{1}, \ldots, j_{q}}}^{m} M_{t_{n}}^{\left(\alpha_{k}\right)^{2}} & \leq \sum_{i=1}^{q} M_{t}^{\left(\alpha_{j_{i}}\right)^{2 m}}+\sum_{\substack{k=1 \\
k \neq j_{1}, \ldots, j_{q}}}^{m} M_{t_{n}}^{\left(\alpha_{k}\right)^{2 m}} \\
& \leq \sum_{i=1}^{q} M_{t}^{\left(\alpha_{j_{j}}\right)^{2 m}}+\sum_{\substack{k=1 \\
k \neq j_{1}, \ldots, j_{q}}}^{m} \sup _{n \geq 1}\left(M_{t_{n}}^{\left(\alpha_{k}\right)^{2 m}}\right)
\end{aligned}
$$

Obviously,

$$
\sup _{n \geq 1}\left(M_{t_{n}}^{\left(\alpha_{k}\right)^{2 m}}\right) \leq \sup _{0 \leq s \leq t}\left(M_{s}^{\left(\alpha_{k}\right)^{2 m}}\right)
$$

and, by Doob's inequality and (4.18), the right-hand side in the previous estimation is integrable. Hence $\left(a_{n}\right)_{n \geq 1}$ is dominated in $L^{1}(\mathbb{P})$ by a quantity which does not depend on $n$. Therefore the theorem of Lebesgue on dominated convergence can be applied to get

$$
0=\lim _{n \rightarrow \infty} \mathbb{E}\left[a_{n}\right]=\mathbb{E}\left[\lim _{n \rightarrow \infty} a_{n}\right]=\mathbb{E}\left[N \prod_{i=1}^{q} M_{t}^{\left(\alpha_{j_{i}}\right)} \prod_{\substack{k=1 \\ k \neq j_{1}, \ldots, j_{q}}}^{m} M_{t-}^{\left(\alpha_{i}\right)}\right]
$$

and this concludes the proof.

Now we come to the main result of this section. We are going to show that if $\mathscr{Z} \subseteq \mathscr{H}_{\mathrm{loc}, 0}^{2}\left(\mathbb{F}_{+}^{\mathscr{Z}}\right)$ is a compensated-covariation stable family of true martingales satisfying (4.17) and such that for every $M^{(\alpha)}$ and $M^{(\beta)}$ the associated point brackets $\left\langle M^{(\alpha)}, M^{(\beta)}\right\rangle$ are deterministic, $\alpha, \beta \in I$, then $\mathscr{Z}$ possesses the $\mathscr{H}^{2}$-PRP with respect to the filtration $\mathbb{F}_{+}^{\mathscr{Z}}$. We recall that by $\mathscr{L}^{2}(\mathscr{Z})$ we denote the stable subspace generated by $\mathscr{Z}$ in $\mathscr{H}_{0}^{2}$.

4.1.9 Theorem. Let $\mathscr{Z}=\left\{M^{(\alpha)}, \alpha \in I\right\}$ be a compensated-covariation stable family of true martingales in $\mathscr{H}_{\mathrm{loc}, 0}^{2}$ on the probability space $\left(\Omega, \mathscr{F}_{\infty}^{\mathscr{Z}}, \mathbb{P}\right)$ and with respect to the filtration $\mathbb{F}_{+}^{\mathscr{Z}}$ which satisfies (4.17). If, for every $\alpha$ and $\beta$ belonging to $I$, the process $\left\langle M^{(\alpha)}, M^{(\beta)}\right\rangle$ is deterministic, then the family $\mathscr{Z}$ has the $\mathscr{H}^{2}-P R P$ with respect to $\mathbb{F}_{+}^{\mathscr{Z}}$, i.e.,

$$
\mathscr{L}^{2}(\mathscr{Z})=\mathscr{H}_{0}^{2}\left(\mathbb{F}_{+}^{\mathscr{Z}}\right) .
$$

Proof. Let $\mathscr{S}:=\mathbb{R}_{+}$be the family of deterministic finite-valued stopping times and $\mathscr{Z}^{\mathscr{S}}$ the family obtained from $\mathscr{Z}$ as in (4.4). Obviously $\mathscr{Z}^{\mathscr{S}}$ is stable under stopping with respect to finite-valued deterministic stopping times and it satisfies 
(4.18) because $\mathscr{Z}$ satisfies (4.17). Hence, because $\mathscr{Z} \subseteq \mathscr{H}_{\mathrm{loc}, 0}^{2}$ is a family of true martingale, we have that $\mathscr{Z}^{\mathscr{S}}$ is in fact contained in $\mathscr{H}_{0}^{2}$. Thanks to Lemma 4.1.2, $\mathscr{Z}^{\mathscr{S}}$ is again a compensated-covariation stable family. Moreover, $\mathscr{L}^{2}\left(\mathscr{Z}^{\mathscr{S}}\right)$ is equal to $\mathscr{L}^{2}(\mathscr{Z})$. Indeed, for every $\alpha \in I$, the increasing sequence $(N)_{N \in \mathbb{N}}$ localizes $M^{(\alpha)}$ to $\mathscr{H}_{0}^{2}$ because $M^{(\alpha)}$ has finite moment of second order (cf. (4.17)). The set $\left\{M^{(\alpha)^{N}}, N \in \mathbb{N}\right\}$ is contained in $\mathscr{Z}^{\mathscr{S}}$ and so $\mathscr{L}^{2}\left(\left\{M^{(\alpha)^{N}}, N \in \mathbb{N}\right\}\right) \subseteq \mathscr{L}^{2}\left(\mathscr{Z}^{\mathscr{S}}\right)$, for every $\alpha \in I$. Lemma 3.4.1 implies

$$
\mathscr{L}^{2}\left(\left\{M^{(\alpha)^{N}}, N \in \mathbb{N}\right\}\right)=\mathscr{L}^{2}\left(M^{(\alpha)}\right)
$$

and, by Theorem 1.4.5, the system $\bigcup_{\alpha \in I} \mathscr{L}^{2}\left(M^{(\alpha)}\right)$ is total in $\mathscr{L}^{2}(\mathscr{Z})$. Hence, by the stability of $\mathscr{L}^{2}\left(\mathscr{Z}^{\mathscr{S}}\right), \mathscr{L}^{2}(\mathscr{Z}) \subseteq \mathscr{L}^{2}\left(\mathscr{Z}^{\mathscr{S}}\right)$. The converse inclusion is immediate and therefore we have $\mathscr{L}^{2}\left(\mathscr{Z}^{\mathscr{S}}\right)=\mathscr{L}^{2}(\mathscr{Z})$. This means that to show that $\mathscr{Z}$ possesses the $\mathscr{H}^{2}$-PRP it is enough to verify that $\mathscr{Z}^{\mathscr{S}}$ possesses the $\mathscr{H}^{2}$-PRP. Summarizing, $\mathscr{Z}^{\mathscr{S}}$ is a compensated-covariation stable family contained in $\mathscr{H}_{0}^{2}$ satisfying (4.18) and the point brackets of elements of $\mathscr{Z}^{\mathscr{S}}$ are a deterministic process. Moreover, $\mathscr{Z}^{\mathscr{S}}$ is stable under stopping with respect to finite-valued deterministic stopping times and generates in $\mathscr{H}_{0}^{2}$ the same stable subspace as $\mathscr{Z}$. Without loss of generality, we can assume for $\mathscr{Z}$ all the properties of $\mathscr{Z}^{\mathscr{S}}$. These further assumptions on the family $\mathscr{Z}$ will considerably simplify the notations in the sequel. Our aim is to show that the compensated-covariation stable family $\mathscr{Z}$ such that $\left\langle M^{(\alpha)}, M^{(\beta)}\right\rangle$ is deterministic, $\alpha, \beta \in I$, with the further assumptions explained before, i.e., $\mathscr{Z} \subseteq \mathscr{H}_{0}^{2}$ fulfills (4.18) and is stable under stopping with respect to deterministic finite-valued stopping times, satisfies condition $C_{2}$ (cf. Definition 1.4.10). In other words, we show that every martingale $N$ belonging to $\mathscr{H}_{0}^{2}$ which is orthogonal to $\mathscr{Z}$ is evanescent. To prove this result we verify that if $N \in \mathscr{H}_{0}^{2}$ is orthogonal to $\mathscr{Z}$, then its terminal variable $N_{\infty}$ is orthogonal in $L^{2}\left(\Omega, \mathscr{F}_{\infty}^{\mathscr{Z}}, \mathbb{P}\right)$ to the system $\mathscr{K}$ defined in $(4.20)$ which is total (cf. Proposition 4.1.6). We proceed by (strong) induction on $m \in \mathbb{N}$. We recall that from (4.18) and (4.19) it follows immediately that $\prod_{i=1}^{m} M_{\infty}^{\left(\alpha_{i}\right)}$ belongs to $L^{2}\left(\Omega, \mathscr{F}_{\infty}^{\mathscr{Z}}, \mathbb{P}\right)$, for every $\alpha_{1}, \ldots, \alpha_{m} \in I$. Let $N \in \mathscr{H}_{0}^{2}\left(\mathbb{F}_{+}^{\mathscr{Z}}\right)$ be such that $N \perp \mathscr{Z}$. If $m=1$, because of the orthogonality, $N M^{(\alpha)} \in \mathscr{H}_{0}^{1}$ (cf. Proposition 1.2.10.(i)) and so

$$
\mathbb{E}\left[N_{\infty} M_{\infty}^{(\alpha)}\right]=\mathbb{E}\left[N_{0} M_{0}^{(\alpha)}\right]=0 .
$$

Now we assume that for every $\alpha_{1}, \ldots, \alpha_{n} \in I, n \leq m$, we have

$$
\mathbb{E}\left[N_{\infty} \prod_{i=1}^{n} M_{\infty}^{\left(\alpha_{i}\right)}\right]=0, \quad n \leq m
$$

From (4.23), it follows that

$$
\mathbb{E}\left[N_{\infty} \prod_{i=1}^{n} M_{t_{i}}^{\left(\alpha_{i}\right)}\right]=0, \quad t_{1}, \ldots, t_{n} \in \mathbb{R}_{+}, \quad n \leq m
$$

Indeed, by assumption, the family $\mathscr{Z}$ is stable under stopping with respect to deterministic finite-valued stopping times, meaning that for every $v \in \mathbb{R}_{+}$the martingale 
$M^{(\alpha)^{v}}$ belongs to $\mathscr{Z}, \alpha \in I$. Therefore, for every $\alpha_{i} \in I$ and $t_{i} \in \mathbb{R}_{+}, i=1, \ldots, n$, $n \leq m$, there exists $\beta_{i} \in I$ such that

$$
M^{\left(\alpha_{i}\right)^{t_{i}}}=M^{\left(\beta_{i}\right)}, \quad i=1, \ldots, n, \quad n \leq m,
$$

and so

$$
\mathbb{E}\left[N_{\infty} \prod_{i=1}^{n} M_{t_{i}}^{\left(\alpha_{i}\right)}\right]=\mathbb{E}\left[N_{\infty} \prod_{i=1}^{n} M_{\infty}^{\left(\alpha_{i}\right)^{t_{i}}}\right]=\mathbb{E}\left[N_{\infty} \prod_{i=1}^{n} M_{\infty}^{\left(\beta_{i}\right)}\right]=0, \quad n \leq m,
$$

where, in the last equality, we used (4.23). Now we prove (4.23) for $m+1$. Because the random variable $N_{\infty}$ belongs to $L^{2}\left(\Omega, \mathscr{F}_{\infty}^{\mathscr{Z}}, \mathbb{P}\right)$, the product $N_{\infty} \prod_{i=1}^{m+1} M_{\infty}^{\left(\alpha_{i}\right)}$ is integrable and from Proposition 4.1.5 we have

$$
\begin{aligned}
\mathbb{E}\left[N_{\infty}\right. & \left.\prod_{i=1}^{m+1} M_{\infty}^{\left(\alpha_{i}\right)}\right] \\
= & \sum_{i=1}^{m+1} \sum_{\substack{1 \leq j_{1}<\ldots<j_{i} \leq m+1\\
}} \mathbb{E}\left[N_{\infty}\left(\left(\prod_{\substack{k=1 \\
k \neq j_{1}, \ldots, j_{i}}}^{m+1} M_{-}^{\left(\alpha_{k}\right)}\right) \cdot M_{\infty}^{\left(\alpha_{j_{1}}, \ldots, \alpha_{j_{i}}\right)}\right)\right] \\
& +\sum_{p=0}^{m-1} \sum_{i=p+2}^{m+1} \sum_{1 \leq j_{1}<\ldots<j_{i} \leq m+1}\left\{\mathbb { E } \left[N _ { \infty } \left(\left(\prod_{\substack{k=1 \\
k \neq j_{1}, \ldots, j_{i}}}^{m+1} M_{-}^{\left(\alpha_{k}\right)}\right) \times\right.\right.\right. \\
& \left.\left.\left.\times\left(\prod_{\ell=j_{i-p+1}}^{j_{i}} \Delta M^{\left(\alpha_{\ell}\right)}\right)\right\} \cdot\left\langle M^{\left(\alpha_{j_{1}}, \ldots, \alpha_{j_{i-p-1}}\right)}, M^{\left(\alpha_{j_{i-p}}\right)}\right\rangle_{\infty}\right)\right] .
\end{aligned}
$$

For the first summand on the right-hand side of (4.25) we have

$$
\sum_{i=1}^{m+1} \sum_{1 \leq j_{1}<\ldots<j_{i} \leq m+1} \mathbb{E}\left[N_{\infty}\left(\left(\prod_{\substack{k=1 \\ k \neq j_{1}, \ldots, j_{i}}}^{m+1} M_{-}^{\left(\alpha_{k}\right)}\right) \cdot M_{\infty}^{\left(\alpha_{j_{1}}, \ldots, \alpha_{j_{i}}\right)}\right)\right]=0 .
$$

Indeed, from Lemma 4.1.7, for every fixed $1 \leq j_{1}<\ldots<j_{i} \leq m$, it follows that:

$$
\left(\prod_{\substack{k=1 \\ k \neq j_{1}, \ldots, j_{i}}}^{m+1} M_{-}^{\left(\alpha_{k}\right)}\right) \in \mathrm{L}^{2}\left(M^{\left(\alpha_{j_{1}}, \ldots, \alpha_{j_{i}}\right)}\right)
$$

where we recall that for a local martingale $M \in \mathscr{M}_{\text {loc, } 0}$, the space $\mathrm{L}^{2}(M)$ was introduced in (1.26). From Theorem 1.4.5.(i) it follows that every stochastic integral with respect to $M^{\left(\alpha_{j_{1}}, \ldots, \alpha_{j_{i}}\right)}$ appearing in (4.26) belongs to $\mathscr{L}^{2}\left(M^{\left(\alpha_{j_{1}}, \ldots, \alpha_{j_{i}}\right)}\right)$. But each element $M^{\left(\alpha_{j_{1}}, \ldots, \alpha_{j_{i}}\right)}$ belongs again to $\mathscr{Z}$, because $\mathscr{Z}$ is a compensated-covariation stable family. From Lemma 1.4.6.(i) we know that if $M^{(\alpha)} \in \mathscr{Z}$, then $N$ is also orthogonal to $\mathscr{L}^{2}\left(M^{(\alpha)}\right)$. Therefore, $N$ is orthogonal to $\mathscr{L}^{2}\left(M^{\left(\alpha_{j_{1}}, \ldots, \alpha_{j_{i}}\right)}\right)$ meaning that $N X \in \mathscr{H}_{0}^{1}$, for every $X$ belonging to $\mathscr{L}^{2}\left(M^{\left(\alpha_{j_{1}}, \ldots, \alpha_{j_{i}}\right)}\right)$ (cf. Proposition 1.2.10.(i)). Hence, because $N_{0} X_{0}=0$, for every $X \in \mathscr{L}^{2}\left(M^{\left(\alpha_{j_{1}}, \ldots, \alpha_{j_{i}}\right)}\right)$, we have

$$
\mathbb{E}\left[N_{\infty}\left(\left(\prod_{\substack{k=1 \\ k \neq j_{1}, \ldots, j_{i}}}^{m+1} M_{-}^{\left(\alpha_{k}\right)}\right) \cdot M_{\infty}^{\left(\alpha_{j_{1}}, \ldots, \alpha_{j_{i}}\right)}\right)\right]=0, \quad 1 \leq i \leq m .
$$


Now we show that the second summation on the right-hand side of (4.25) vanishes. The processes $M^{\left(\alpha_{j_{i-p}}\right)}$ and $M^{\left(\alpha_{j_{1}}, \ldots, \alpha_{j_{i-p-1}}\right)}$ belong to $\mathscr{Z}$ for every $i$ and $p$, because $\mathscr{Z}$ is compensated-covariation stable. By assumption, $\left\langle M^{\left(\alpha_{j_{1}}, \ldots, \alpha_{j_{i-p-1}}\right)}, M^{\left(\alpha_{j_{i-p}}\right)}\right\rangle$ are all deterministic. Moreover, from Lemma 4.1.7, we have that all the integrals of the form $\left(\prod_{k \neq j_{1}, \ldots, j_{i}}^{m+1} M_{-}^{\left(\alpha_{k}\right)}\right)\left(\prod_{\ell=j_{i-p+1}}^{j_{i}} \Delta M^{\left(\alpha_{\ell}\right)}\right) \cdot\left\langle M^{\left(\alpha_{j_{1}}, \ldots, \alpha_{j_{i-p-1}}\right)}, M^{\left(\alpha_{j_{i-p}}\right)}\right\rangle$ are processes of integrable variation. We can then apply the theorem of Fubini to get

$$
\begin{aligned}
\sum_{p=0}^{m-1} \sum_{i=p+2}^{m+1} \sum_{1 \leq j_{1}<\ldots<j_{i} \leq m+1}\left\{\mathbb { E } \left[N _ { \infty } \left(\left(\prod_{\substack{k=1 \\
k \neq j_{1}, \ldots, j_{i}}}^{m+1} M_{-}^{\left(\alpha_{k}\right)}\right) \times\right.\right.\right. \\
\left.\left.\left.\times\left(\prod_{\ell=j_{i-p+1}}^{j_{i}} \Delta M^{\left(\alpha_{\ell}\right)}\right)\right\} \cdot\left\langle M^{\left(\alpha_{j_{1}}, \ldots, \alpha_{j_{i-p-1}}\right)}, M^{\left(\alpha_{j_{i-p}}\right)}\right\rangle_{\infty}\right)\right] \\
=\sum_{p=0}^{m-1} \sum_{i=p+2}^{m+1} \sum_{\substack{1 \leq j_{1}<\ldots<j_{i} \leq m+1\\
}}\left\{\mathbb { E } \left[N _ { \infty } \left(\left(\prod_{\substack{k=1 \\
k \neq j_{1}, \ldots, j_{i}}}^{m+1} M_{-}^{\left(\alpha_{k}\right)}\right) \times\right.\right.\right. \\
\left.\left.\left.\times\left(\prod_{\ell=j_{i-p+1}}^{j_{i}} \Delta M^{\left(\alpha_{\ell}\right)}\right)\right)\right]\right\} \cdot\left\langle M^{\left(\alpha_{j_{1}}, \ldots, \alpha_{j_{i-p-1}}\right)}, M^{\left(\alpha_{j_{i-p}}\right)}\right\rangle_{\infty} .
\end{aligned}
$$

Let us fix $p$ and $i, 0 \leq p \leq m-1, p+2 \leq i \leq m+1$ and $1 \leq j_{1}<\ldots<j_{i} \leq m+1$. We consider the generic element

$$
K:=\left(\prod_{\substack{k=1 \\ k \neq j_{1}, \ldots, j_{i}}}^{m+1} M_{-}^{\left(\alpha_{i}\right)}\right)\left(\prod_{\ell=j_{i-p+1}}^{j_{i}} \Delta M^{\left(\alpha_{\ell}\right)}\right) .
$$

The first product appearing in the definition of $K$ has $(m+1)-i$ factors, while the second product has $p+1$ factors, if $p \geq 1$ and it vanishes if $p=0$. Therefore, the process $K$ consists of $(m+1)+(p+1-i)$ factors. On the other hand, $i \geq p+2$ and so $(p+1-i) \leq 0$. In conclusion, $K$ consists of, at most, $m$ factors. Also each product appearing in the definition of $K$ has not more than $m$ factors, so we can use the induction hypothesis. After expanding the product we observe that $K_{t}$ is equal to a finite sum of terms of type

$\prod_{k=1}^{q} M_{t-}^{\left(\alpha_{i_{k}}\right)} \prod_{j=q+1}^{q+r} M_{t}^{\left(\alpha_{i_{j}}\right)}, \quad 1 \leq i_{1}, \ldots, i_{q+r} \leq m+1$ pairwise different, $\quad q+r \leq m-1$.

From the induction hypothesis, (4.24) and Lemma 4.1.8, it follows that $\mathbb{E}\left[N_{\infty} K_{t}\right]=$ 0 . Then (4.27) vanishes. This, together with (4.26) and (4.25), implies that

$$
\mathbb{E}\left[N_{\infty} \prod_{i=1}^{m+1} M_{\infty}^{\left(\alpha_{i}\right)}\right]=0
$$

Hence, because of the induction, we can conclude that if $N \perp \mathscr{Z}$, then for every $m \in \mathbb{N}$ and every $\alpha_{1}, \ldots, \alpha_{m} \in I$ it follows:

$$
\mathbb{E}\left[N_{\infty} \prod_{i=1}^{m} M_{\infty}^{\left(\alpha_{i}\right)}\right]=0
$$


The family $\mathscr{Z}$ is assumed to be stable under stopping with respect to finite-valued deterministic stopping times and this yields:

$$
\mathbb{E}\left[N_{\infty} \prod_{i=1}^{m} M_{t_{i}}^{\left(\alpha_{i}\right)}\right]=0, \quad t_{1}, \ldots, t_{m} \in \mathbb{R}_{+}, \quad \alpha_{1}, \ldots, \alpha_{m} \in \mathbb{R}_{+}, \quad m \in \mathbb{N} .
$$

Therefore if $N$ is (strongly) orthogonal to $\mathscr{Z}$, then $N_{\infty}$ is orthogonal (in the sense of the Hilbert space $\left.L^{2}\left(\Omega, \mathscr{F}_{\infty}^{\mathscr{Z}}, \mathbb{P}\right)\right)$ to $\mathscr{K}$, where $\mathscr{K}$ is the family of monomials defined in (4.20). But $\mathscr{K}$ is a total system in $L^{2}\left(\Omega, \mathscr{F}_{\infty}^{\mathscr{Z}}, \mathbb{P}\right)$ (cf. Proposition 4.1.6) and so $N_{\infty}=0$. From Jacod \& Shiryaev (2000), Theorem I.1.42 we know that $N_{t}=\mathbb{E}\left[N_{\infty} \mid \mathscr{F}_{t+}^{\mathscr{Z}}\right]$ and so we get $N_{t}=0$, a.s. for every $t \geq 0$. By our definition of martingale, the process $N$ is càdlàg, thus we can deduce that $N$ is indistinguishable from the identically null process. We have proven that, if $N \in \mathscr{H}_{0}^{2}\left(\mathbb{F}_{+}^{\mathscr{Z}}\right)$ is orthogonal to $\mathscr{Z}$, then it is evanescent, or equivalently that $\mathscr{Z}$ satisfies condition $C_{2}$. Theorem 1.4.11.(ii) implies that the family $\mathscr{Z}$ possesses the $\mathscr{H}^{2}$-PRP with respect to $\mathbb{F}_{+}^{\mathscr{Z}}$ and this completes the proof.

We now give first examples of compensated-covariation stable families fulfilling all the assumptions of Theorem 4.1.9 and hence possessing the $\mathscr{H}^{2}$-PRP.

\subsubsection{Continuous Gaussian Families}

We start by considering a Wiener process $W$ on the probability space $\left(\Omega, \mathscr{F}_{\infty}^{W}, \mathbb{P}\right)$, where $\mathscr{F}_{\infty}^{W}:=\sigma\left(W_{t}, t \geq 0\right) \vee \mathscr{N}(\mathbb{P})$ and $\mathscr{N}(\mathbb{P})$ are the null-sets of $\sigma\left(W_{t}, t \geq 0\right)$. We refer to the natural filtration of $W, \mathbb{F}^{W}=\left(\mathscr{F}_{t}^{W}\right)_{t \geq 0}$. Obviously, $[W, W]-\langle W, W\rangle=0$ and so the family $\mathscr{Z}:=\{W\} \cup\{0\}$ is a compensated-covariation stable family satisfying all the assumptions of Theorem 4.1.9. Therefore

$$
\mathscr{L}^{2}(W)=\mathscr{L}^{2}(\mathscr{Z})=\mathscr{H}^{2}\left(\mathbb{F}_{+}^{\mathscr{Z}}\right)=\mathscr{H}^{2}\left(\mathbb{F}^{W}\right) .
$$

In other words, from Theorem 4.1.9, we can deduce the very well-known fact (cf. Clark (1970)) that a Wiener process $W$ possesses the $\mathscr{H}^{2}$-PRP with respect to $\mathbb{F}^{W}$.

More generally, on a complete probability space $(\Omega, \mathscr{F}, \mathbb{P})$ and with a filtration $\mathbb{F}$ satisfying the usual conditions, we consider a family $\mathscr{X}:=\left\{M^{(\alpha)}, \alpha \in I\right\}$ of continuous local martingales such that $M_{0}^{(\alpha)}=0$ and that $\left\langle M^{(\alpha)}, M^{(\beta)}\right\rangle$ is a deterministic (continuous) function, for every $\alpha$ and $\beta$ in $I$. We are going to show that such a family $\mathscr{X}$ of continuous local martingales is a Gaussian family, i.e., for every $\alpha_{1}, \ldots, \alpha_{m} \in I$ and for every $t_{1}^{\left(\alpha_{i}\right)}, \ldots, t_{n_{\alpha_{i}}}^{\left(\alpha_{i}\right)} \geq 0, i=1, \ldots, m$, the vector

$$
\left(M_{t_{1}^{\left(\alpha_{1}\right)}}^{\left(\alpha_{1}\right)}, \ldots, M_{t_{n_{\alpha_{1}}}^{\left(\alpha_{1}\right)}}^{\left(\alpha_{1}\right)} ; \ldots ; M_{t_{1}^{\left(\alpha_{m}\right)}}^{\left(\alpha_{m}\right)}, \ldots, M_{t_{n_{\alpha_{m}}}^{\left(\alpha_{m}\right)}}^{\left(\alpha_{m}\right)}\right)
$$

is Gaussian distributed with covariance function

$$
\operatorname{Cov}\left(M_{t}^{(\alpha)}, M_{s}^{(\beta)}\right)=\left\langle M^{(\alpha)}, M^{(\beta)}\right\rangle_{t \wedge s}, \quad s, t \geq 0 .
$$

This result can be considered as a generalization of the theorem of P. Lévy on the characterization of the Wiener process (cf. Theorem 1.2.9). 
4.1.10 Proposition. A family of continuous local martingales $\mathscr{X}:=\left\{M^{(\alpha)}, \alpha \in I\right\}$ such that $M_{0}^{(\alpha)}=0, \alpha \in I$, is a Gaussian family with covariance structure as in (4.28) if and only if $\left\langle M^{(\alpha)}, M^{(\beta)}\right\rangle$ is a continuous deterministic process, for every $\alpha$ and $\beta$ belonging to $I$.

Proof. If $\mathscr{X}$ is a Gaussian family of continuous local martingales, then $\left\langle M^{(\alpha)}, M^{(\beta)}\right\rangle$ is clearly a continuous deterministic process, for every $\alpha$ and $\beta$ belonging to $I$. We now show the converse implication. It is enough to verify that for every $0=t_{0}<$ $t_{1}<\ldots<t_{n}, n \in \mathbb{N}$, the vector

$$
\left(M_{t_{1}}^{\left(\alpha_{1}\right)}, \ldots, M_{t_{n}}^{\left(\alpha_{1}\right)} ; \ldots ; M_{t_{1}}^{\left(\alpha_{m}\right)}, \ldots, M_{t_{n}}^{\left(\alpha_{m}\right)}\right)
$$

is Gaussian with the following covariance structure:

$$
\operatorname{Cov}\left(M_{t_{i}}^{\left(\alpha_{j}\right)}, M_{t_{\ell}}^{\left(\alpha_{k}\right)}\right)=\left\langle M^{\left(\alpha_{j}\right)}, M^{\left(\alpha_{k}\right)}\right\rangle_{t_{i} \wedge t_{\ell}}, \quad j, k=1, \ldots, m ; \quad i, \ell=1, \ldots, n .
$$

We verify, without loss of generality, that for every $t \geq 0$ the random vector $\left(M_{t}^{\left(\alpha_{1}\right)}, \ldots, M_{t}^{\left(\alpha_{m}\right)}\right)$ is Gaussian and its covariance function is $\operatorname{Cov}\left(M_{t}^{(\alpha)}, M_{t}^{(\beta)}\right)=$ $\left\langle M^{\left(\alpha_{\ell}\right)}, M^{\left(\alpha_{k}\right)}\right\rangle_{t}$, for every $m \in \mathbb{N}$. Indeed, we can assume that the family $\mathscr{X}$ is stable under stopping with respect to finite-valued deterministic stopping times. If it is not the case we can consider the family $\mathscr{X}^{\mathscr{S}}$ defined as in (4.4), where $\mathscr{S}=\mathbb{R}_{+}$ is the collection of all deterministic finite-valued stopping times. This simplifies considerably the notations of the proof. We define the process $Z:=\left(Z_{t}\right)_{t \geq 0}$ by

$$
Z_{t}:=\exp \left(i \sum_{j=1}^{m} M_{t}^{\left(\alpha_{j}\right)}+\frac{1}{2} \sum_{\ell, k=1}^{m} u_{\ell} u_{k}\left\langle M^{\left(\alpha_{\ell}\right)}, M^{\left(\alpha_{k}\right)}\right\rangle_{t}\right)
$$

Obviously, $Z_{0}=1$ and, because $M^{(\alpha)}$ is continuous, $\alpha \in I$, and the process $\left\langle M^{\left(\alpha_{\ell}\right)}, M^{\left(\alpha_{k}\right)}\right\rangle$ is deterministic and continuous, $\ell, k=1, \ldots, m, Z$ is a bounded process on every compact interval. Moreover, applying Itô's, formula we get

$$
Z_{t}=1+\sum_{j=1}^{m} u_{j} Z \cdot M_{t}^{\left(\alpha_{j}\right)}, \quad t \geq 0
$$

Hence the process $Z$ is a local martingale (notice that because of the continuity, $Z$ is a locally bounded predictable process and so $Z \in \mathrm{L}_{\text {loc }}^{q}(M), q \geq 1$, for every $\left.M \in \mathscr{M}_{\text {loc }}\right)$. But because $Z$ is bounded over compact intervals, it is in fact a true martingale. Then we obtain $\mathbb{E}\left[Z_{t}\right]=\mathbb{E}\left[Z_{0}\right]=1$, which, for every $u_{k}, u_{\ell} \in \mathbb{R}$, $k, \ell=1, \ldots, m$, implies that

$$
\mathbb{E}\left[\exp \left(i \sum_{j=1}^{m} M_{t}^{\left(\alpha_{j}\right)}\right)\right]=\exp \left(-\frac{1}{2} \sum_{\ell, k=1}^{m} u_{\ell} u_{k}\left\langle M^{\left(\alpha_{\ell}\right)}, M^{\left(\alpha_{k}\right)}\right\rangle_{t}\right), \quad m \in \mathbb{N}, \quad t \geq 0 .
$$

In particular, from this it follows that the vector $\left(M_{t}^{\left(\alpha_{1}\right)}, \ldots, M_{t}^{\left(\alpha_{m}\right)}\right)$ is Gaussian with covariance function $\operatorname{Cov}\left(M_{t}^{(\alpha)}, M_{t}^{(\beta)}\right)=\left\langle M^{\left(\alpha_{\ell}\right)}, M^{\left(\alpha_{k}\right)}\right\rangle_{t}$, for every $m \in \mathbb{N}$ and the proof is finished. 
We consider a family $\mathscr{X}:=\left\{M^{(\alpha)}, \alpha \in I\right\}$ of continuous local martingales such that the process $\left\langle M^{(\alpha)}, M^{(\beta)}\right\rangle$ is deterministic, for every $\alpha, \beta \in I$. We restrict our attention to the probability space $\left(\Omega, \mathscr{F}_{\infty}, \mathbb{P}\right)$ and to the filtration $\mathbb{F}_{+}^{\mathscr{X}}$. Because of Theorem 1.2.9, we know that $M^{(\alpha)}$ is a Wiener process relative to $\mathbb{F}_{+}^{\mathscr{X}}$ with variance function $\sigma_{\alpha}^{2}(t)=\left\langle M^{(\alpha)}, M^{(\alpha)}\right\rangle_{t}$, for every $\alpha \in I$ and $t \geq 0$. Therefore $\mathscr{X}$ is a family of true martingales. Moreover, for every $\alpha \in I$ and for every $t \geq 0$, the random variable $M_{t}^{(\alpha)}$ satisfies condition (4.17). Thanks to the continuity of $M^{(\alpha)}$, $\alpha \in I$, we have that, for every $\alpha, \beta \in I$ the compensated-covariation process $M^{(\alpha, \beta)}$ is identically equal to zero and therefore the family $\mathscr{Z}:=\mathscr{X} \cup\{0\}$ is a compensated covariation-stable family which satisfies all the assumptions of Theorem 4.1.9. From this follows that

$$
\mathscr{L}^{2}(\mathscr{X})=\mathscr{L}^{2}(\mathscr{Z})=\mathscr{H}_{0}^{2}\left(\mathbb{F}_{+}^{\mathscr{X}}\right)
$$

i.e., $\mathscr{X}$ has the $\mathscr{H}^{2}$-PRP with respect to $\mathbb{F}_{+}^{\mathscr{X}}$. Notice that because of the continuity of the family $\mathscr{X}$, thanks to Jacod (1979), Proposition 4.13 and Theorem 1.4.11, we can even conclude that the Gaussian family $\mathscr{X}$ has the $\mathscr{H}^{q}$-PRP with respect to $\mathbb{F}_{+}^{\mathscr{X}}$, for every $q \geq 1$. In conclusion we have shown that every Gaussian family $\mathscr{X}$ possesses the $\mathscr{H}^{q}-\mathrm{PRP}$ with respect to $\mathbb{F}_{+}^{\mathscr{X}}$, for every $q \geq 1$. We stress that fromJacod (1979), Proposition 4.13 we can deduce that $\mathscr{X}$ satisfies condition $C_{1}$. Indeed, $\mathscr{X}$ has the $\mathscr{H}^{2}$-PRP and so it satisfies condition $C_{2}$. Because of the continuity of the martingales in $\mathscr{X}$, this is equivalent to condition $C_{1}$.

\subsubsection{Independent Families of Poisson Processes}

In Kabanov (1973) and Kabanov (1974) it was proven that a compensated Poisson process $\bar{N}$ possesses the $\mathscr{H}^{2}$-PRP with respect to its natural augmented filtration $\mathbb{F}^{N}$. We now verify once again this result as a consequence of Theorem 4.1.9. We consider a Poisson process $N$ on the probability space $\left(\Omega, \mathscr{F}_{\infty}^{N}, \mathbb{P}\right)$, where $\mathscr{F}_{\infty}^{N}:=$ $\sigma\left(N_{t}, t \geq 0\right) \vee \mathscr{N}(\mathbb{P})$ and $\mathscr{N}(\mathbb{P})$ are the null-sets of $\sigma\left(N_{t}, t \geq 0\right)$. Let $a(\cdot)$ be the intensity function of $N$, which is a continuous deterministic increasing function. We define the family $\mathscr{Z}$ by $\mathscr{Z}:=\{\bar{N}\}$, where $\bar{N}:=N-a(\cdot)$. We have

$$
[\bar{N}, \bar{N}]-\langle\bar{N}, \bar{N}\rangle=\sum_{0<s \leq t}\left(\Delta N_{s}\right)^{2}-a(\cdot)=\sum_{0<s \leq t} \Delta N_{s}-a(\cdot)=\bar{N} .
$$

All the assumptions of Theorem 4.1.9 are satisfied and so we can conclude that the family $\mathscr{Z}$ has the $\mathscr{H}^{2}$-PRP with respect to $\mathbb{F}^{N}=\mathbb{F}^{\bar{N}}$. More generally, for a family $\mathscr{X}:=\left\{M^{(\alpha)}, \alpha \in I\right\}$ of independent compensated Poisson processes, on the probability space $\left(\Omega, \mathscr{F}_{\infty}^{\mathscr{X}}, \mathbb{P}\right)$ and with respect to the filtration $\mathbb{F}_{+}^{\mathscr{X}}$, we have that, for every $\alpha, \beta \in I$ such that $\alpha \neq \beta$, the process $\left[M^{(\alpha)}, M^{(\beta)}\right]$ is equal to zero (cf. Theorem A.1.1). This implies that also the process $\left\langle M^{(\alpha)}, M^{(\beta)}\right\rangle$ is equal to zero. Therefore, thanks to Theorem 4.1.9, the compensated-covariation stable family $\mathscr{Z}:=\mathscr{X} \cup\{0\}$ possesses the $\mathscr{H}^{2}$-PRP with respect to $\mathbb{F}_{+}^{\mathscr{Z}}=\mathbb{F}_{+}^{\mathscr{X}}$.

We do not go on discussing further examples. Rather we demand this task to $\S 4.2$, where, using the theory developed in this section, we shall construct families of martingales with the $\mathscr{H}^{2}$-PRP with respect to the natural filtration of a Lévy process. 


\subsection{Martingales of the Natural Filtration of a Lévy Process}

In this section we apply the theory developed in the previous one to Lévy processes. More precisely, given a Lévy process $L$ with respect to the filtration $\mathbb{F}^{L}$ on the probability space $\left(\Omega, \mathscr{F}_{\infty}^{L}, \mathbb{P}\right)$, we construct a family $\mathscr{Z}$ of compensated-covariation stable $\mathbb{F}^{L}$-martingales satisfying the assumptions of Theorem 4.1.9. As usual, for a Lévy process $\left(L, \mathbb{F}^{L}\right)$ with Lévy measure $\nu$, we denote by $\mathrm{M}$ its jump measure. In $\S 2.3 .1$ we have shown that $\mathrm{M}$ is a Poisson random measure relative to the filtration $\mathbb{F}^{L}$ and that its intensity measure is given by $\lambda_{+} \otimes \nu$, where $\lambda_{+}$is the Lebesgue measure on $\left(\mathbb{R}_{+}, \mathscr{B}\left(\mathbb{R}_{+}\right)\right)$and $\nu$ the Lévy measure of $L$. By $\overline{\mathrm{M}}$ we denote the compensated Poisson random measure of $L$, i.e., we put $\overline{\mathrm{M}}:=\mathrm{M}-\lambda_{+} \otimes \nu$. In $\S 2.2 .5$, for $f \in L^{2}(\nu)$, we have introduced the process $X^{(f)}=\left(X_{t}^{(f)}\right)_{t \geq 0}$ by

$$
X_{t}^{(f)}:=\overline{\mathrm{M}}\left(1_{[0, t]} f\right), \quad t \geq 0 .
$$

In Theorem 2.2.16 we have shown that $X^{(f)}$ is a true martingale in $\mathscr{H}_{\mathrm{loc}, 0}^{2}$ and that for any deterministic stopping time $v \geq 0$, the stopped process $X^{(f)^{v}}$ belongs to $\mathscr{H}_{0}^{2}$. Moreover, $\left\langle X^{(f)}, X^{(g)}\right\rangle$ is given by $\left\langle X^{(f)}, X^{(g)}\right\rangle_{t}=t \nu(f g)$. If the function $f$ belongs to $L^{1}(\nu) \cap L^{2}(\nu)$, then the martingale $X^{(f)}$ is of finite variation and we have the identity (cf. Proposition 2.2.12)

$$
X_{t}^{(f)}:=\mathrm{M}\left(1_{[0, t]} f\right)-t \nu(f)=\sum_{0 \leq s \leq t} f\left(\Delta L_{s}\right) 1_{\left\{\Delta L_{s} \neq 0\right\}}-t \nu(f) \quad \text { a.s., } \quad t \geq 0 .
$$

4.2.1 Assumption. Let $\mathscr{T} \subseteq L^{2}(\nu)$ be a system of functions satisfying the following properties:

(i) $\mathscr{T} \subseteq L^{1}(\nu) \cap L^{2}(\nu)$.

(ii) $\mathscr{T}$ is total in $L^{2}(\nu)$.

(iii) $\mathscr{T}$ is stable under multiplication.

(iv) $\mathscr{T}$ is a system of bounded functions.

We observe that, given a Lévy measure $\nu$, a system $\mathscr{T} \subseteq L^{2}(\nu)$ with the properties of Assumptions 4.2.1 always exists. For example, the system

$$
\mathscr{T}:=\left\{1_{(a, b]}, \quad a, b \in \mathbb{R}: a<b, 0 \notin[a, b]\right\}
$$

is contained in $L^{1}(\nu) \cap L^{2}(\nu)$ and is stable under multiplication. Moreover, it satisfies the assumptions in Lemma 1.1.8 and therefore it is total in $L^{2}(\nu)$. Let $\mathscr{T} \subseteq L^{2}(\nu)$ be as in Assumption 4.2.1 and $\mathrm{W}^{\sigma}$ denote the Gaussian part of the process $L$. We define the family $\mathscr{Z}$ as it follows:

$$
\mathscr{Z}:=\left\{\mathrm{W}^{\sigma}\right\} \cup\left\{X^{(f)}, \quad f \in \mathscr{T}\right\} \cup\{0\} .
$$

At first, we show that the filtration $\mathbb{F}_{+}^{\mathscr{Z}}$ coincides with $\mathbb{F}^{L}$. Because of the rightcontinuity of $\mathbb{F}^{L}$, it suffices to show that the filtration $\mathbb{F}^{\mathscr{Z}}=\left(\mathscr{F}_{t}^{\mathscr{Z}}\right)_{t \geq 0}$, where we set $\mathscr{F}_{t}^{\mathscr{Z}}:=\sigma\left(M_{s} 0 \leq s \leq t, M \in \mathscr{Z}\right) \vee \mathscr{N}(\mathbb{P}), t \geq 0$, and $\mathscr{N}(\mathbb{P})$ denotes the collection of the null-sets of $\sigma\left(L_{t}, t \geq 0\right)$, is equal to $\mathbb{F}^{L}$. In $\S 2.3 .3$ we have discussed the 
structure of the filtration $\mathbb{F}^{L}$. In particular, from Proposition 2.3.11, we know that the identity

$$
\mathbb{F}^{L}=\mathbb{F}^{\mathrm{W}^{\sigma}} \vee \mathbb{F}^{\mathrm{M}}
$$

holds, where $\mathbb{F}^{\mathrm{W}^{\sigma}}$ is the natural filtration of the Wiener process $\mathrm{W}^{\sigma}$ while $\mathbb{F}^{\mathrm{M}}$ is the natural filtration of $\mathrm{M}$, introduced in $\S 2.3 .3$. So,

$$
\mathscr{F}_{t}^{L}=\mathscr{F}_{t}^{\mathrm{W}^{\sigma}} \vee \mathscr{F}_{t}^{\mathrm{M}}, \quad t \geq 0 .
$$

By definition of $\mathbb{F}^{\mathscr{Z}}$, we have

$$
\left.\mathbb{F}^{\mathscr{Z}}:=\mathbb{F}^{\mathrm{W}^{\sigma}} \vee \mathbb{F}^{\left\{X^{(f)}\right.}, f \in \mathscr{T}\right\} .
$$

Now we show that $\mathbb{F}^{\left\{X^{(f)}, f \in \mathscr{T}\right\}}$ equals $\mathbb{F}^{\mathrm{M}}$. We verify $\mathscr{F}_{\infty}^{\left\{X^{(f)}, f \in \mathscr{T}\right\}}=\mathscr{F}_{\infty}^{\mathrm{M}}$. The proof that $\mathscr{F}_{t}^{\left\{X^{(f)}, f \in \mathscr{T}\right\}}$ is equal to $\mathscr{F}_{t}^{\mathrm{M}}$ for every $t \geq 0$ is analogous.

4.2.2 Lemma. $\mathscr{F}_{\infty}^{\left\{X^{(f)}, f \in \mathscr{T}\right\}}=\mathscr{F}_{\infty}^{\mathrm{M}}$.

Proof. Because of Proposition 2.3.10, each martingale $X^{(f)}$, with $f \in \mathscr{T}$, is $\mathbb{F}^{\mathrm{M}}$ adapted. Because each $X^{(f)}$ is also càdlàg, we can conclude that it is a $\mathscr{B}\left(\mathbb{R}_{+}\right) \otimes \mathscr{F}_{\infty^{-}}^{\mathrm{M}}$ measurable process. Therefore $X_{t}^{(f)}$ is $\mathscr{F}_{\infty}^{\mathrm{M}}$-measurable for every $t \geq 0$ and $f \in \mathscr{T}$. Hence $\mathscr{F}_{\infty}^{\left\{X^{(f)}, f \in \mathscr{T}\right\}} \subseteq \mathscr{F}_{\infty}^{\mathrm{M}}$. We have to show the converse inclusion. Let $\mathscr{C}$ be the system defined by $\mathscr{C}=\{(u, v] \times(a, b], u, v \geq 0,0 \notin[a, b]\}$. In Lemma 2.3.9 we proved that $\mathscr{C}^{\mathrm{M}}:=\{\mathrm{M}(A), A \in \mathscr{C}\}$ generates the $\sigma$-algebra $\mathscr{F}_{\infty}^{\mathrm{M}}$. So, to conclude, it is enough to show that the system $\mathscr{C}_{\mathrm{M}}$ is $\mathscr{F}_{\infty}^{\left\{X^{(f)}, f \in \mathscr{T}\right\}}$-measurable. For this purpose, we take $A \in \mathscr{C}$ of the form $A=(u, v] \times(a, b]$, where $0 \leq u<v$ and $a<b$ such that $0 \notin[a, b]$. For such an $A$ we have $\mathrm{M}(A)=\mathrm{M}\left(1_{[0, v]} 1_{(a, b]}\right)-\mathrm{M}\left(1_{[0, u]} 1_{(a, b]}\right)$ and so it is sufficient to verify that $\mathrm{M}\left(1_{[0, t]} 1_{(a, b]}\right)$ is $\mathscr{F}_{\infty}^{\left\{X^{(f)}, f \in \mathscr{T}\right\}}$-measurable for every $t \geq 0$. The function $1_{(a, b]}$ belongs to $L^{2}(\nu)$ because $0 \notin[a, b]$. The system $\mathscr{T}$ is total in $L^{2}(\nu)$ (cf. Assumption 4.2.1). Therefore there exists a sequence $\left(f_{n}\right)_{n \geq 1} \subseteq \operatorname{Span}(\mathscr{T})$ such that $f_{n} \longrightarrow 1_{(a, b]}$ in $L^{2}(\nu)$ as $n \rightarrow+\infty$. By linearity and isometry we have

$$
\left\|X_{t}^{\left(f_{n}\right)}-X_{t}^{\left(1_{(a, b]}\right)}\right\|_{2}^{2}=\left\|X_{t}^{\left(f_{n}-1_{(a, b]}\right)}\right\|_{2}^{2}=t\left\|f_{n}-1_{(a, b]}\right\|_{L^{2}(\nu)}^{2} \longrightarrow 0 \quad \text { as } n \rightarrow+\infty .
$$

Consequently, $X_{t}^{\left(f_{n}\right)}$ converges in $L^{2}\left(\Omega, \mathscr{F}_{\infty}^{L}, \mathbb{P}\right)$ to $X_{t}^{\left(1_{(a, b]}\right)}$, for every $t \geq 0$. But the random variable $X_{t}^{\left(f_{n}\right)}$ is $\tilde{\mathscr{F}}_{\infty}^{\left\{X^{(f)}, f \in \mathscr{T}\right\}}$-measurable, for every fixed $t \geq 0$, and so $X^{\left(1_{(a, b]}\right)}$ is $\mathscr{F}_{\infty}^{\left\{X^{(f)}, f \in \mathscr{T}\right\}}$-measurable. On the other side, $1_{(a, b]}$ belongs also to $L^{1}(\nu)$ which yields the relation

$$
X_{t}^{\left(1_{(a, b]}\right)}=\mathrm{M}\left(1_{[0, t]} 1_{(a, b]}\right)-t \nu((a, b]) \quad \text { a.s., } \quad t \geq 0 .
$$

Hence $\mathrm{M}\left(1_{[0, t]} 1_{(a, b]}\right)$ is $\mathscr{F}_{\infty}^{\left\{X^{(f)}, f \in \mathscr{T}\right\}}$-measurable too and so $\mathscr{C}^{\mathrm{M}}$ is $\mathscr{F}_{\infty}^{\left\{X^{(f)}, f \in \mathscr{T}\right\}}$ measurable, which yields $\mathscr{F}_{\infty}^{\mathrm{M}} \subseteq \mathscr{F}_{\infty}^{\left\{X^{(f)}, f \in \mathscr{T}\right\}}$.

Now we prove that the family $\mathscr{Z}$ in (4.29) satisfies the assumptions of Theorem 4.1.9. 
4.2.3 Proposition. The family $\mathscr{Z}$ in $(4.29)$ is a compensated-covariation stable family of $\mathbb{F}^{L}$-martingales in $\mathscr{H}_{\mathrm{loc}, 0}^{2}$. Moreover, for every $M \in \mathscr{Z}$ and for every $c>0$, we have

$$
\mathbb{E}\left[\exp \left(c\left|M_{t}\right|\right)\right]<+\infty, \quad t \geq 0,
$$

and for every $N \in \mathscr{Z}$, the predictable process of finite variation $\langle M, N\rangle$ is deterministic and continuous.

Proof. It is clear that $\mathscr{Z} \subseteq \mathscr{H}_{\mathrm{loc}, 0}^{2}$. Furthermore, because each function $f$ in $\mathscr{T}$ is bounded, from Theorem 2.2.16.(iv) it follows that each martingale $X^{(f)}$ is locally bounded. The process $\mathrm{W}^{\sigma}$ is continuous and so locally bounded. Consequently, $\mathscr{Z}$ is a family of locally bounded Lévy processes and Theorem 2.3.8.(iv) ensures (4.31). The process $\left\langle\mathrm{W}^{\sigma}, \mathrm{W}^{\sigma}\right\rangle$ is given by $\left\langle\mathrm{W}^{\sigma}, \mathrm{W}^{\sigma}\right\rangle_{t}=\sigma^{2} t$. For every $f \in \mathscr{T}$ we have $\left\langle\mathrm{W}^{\sigma}, X^{(f)}\right\rangle=0$ and if $f, g \in \mathscr{T}$, then $\left\langle X^{(f)}, X^{(g)}\right\rangle_{t}=\nu(f g) t$. Therefore $\langle M, N\rangle$ is a deterministic continuous process of finite variation, for every $M, N \in \mathscr{Z}$. It remains to show that $\mathscr{Z}$ is a compensated-covariation stable family. We have that $\left[\mathrm{W}^{\sigma}, \mathrm{W}^{\sigma}\right]-\left\langle\mathrm{W}^{\sigma}, \mathrm{W}^{\sigma}\right\rangle=0$ and $\left[\mathrm{W}^{\sigma}, X^{(f)}\right]-\left\langle\mathrm{W}^{\sigma}, X^{(f)}\right\rangle=0, f \in \mathscr{T}$, because $\mathrm{W}^{\sigma}$ is a continuous martingale and $X^{(f)}$ a purely discontinuous martingale (cf. $\S 1.2 .7$ and $\S 1.2 .8)$ and so these processes belong all to $\mathscr{Z}$ by definition. Now we compute the compensated-covariation process $X^{(f, g)}$ for $f, g \in \mathscr{T}$. Because $\mathscr{T}$ is stable under multiplication, the function $f g$ belongs to $\mathscr{T}$, for every $f, g \in \mathscr{T}$. Therefore the martingale $X^{(f g)}$ can be defined and it belongs to $\mathscr{Z}$. By Theorem 2.2.16, we have

$$
\begin{aligned}
X_{t}^{(f, g)} & :=\left[X^{(f)}, X^{(g)}\right]_{t}-\left\langle X^{(f)}, X^{(g)}\right\rangle_{t} \\
& =\sum_{0<s \leq t} \Delta X_{s}^{(f)} \Delta X_{s}^{(g)}-\nu(f g) t \\
& =\sum_{0<s \leq t} f\left(\Delta L_{s}\right) g\left(\Delta L_{s}\right) 1_{\left\{\Delta L_{s} \neq 0\right\}}-\nu(f g) t \\
& =\mathrm{M}\left(1_{[0, t]} f g\right)-\nu(f g) t=\overline{\mathrm{M}}\left(1_{[0, t]} f g\right)=: X_{t}^{(f g)}, \quad t \geq 0, \quad \text { a.s., },
\end{aligned}
$$

where, in the last equality, we used that $f g \in L^{1}(\nu) \cap L^{2}(\nu)$ and Proposition 2.2.12. In other words, we obtained the identity

$$
X_{t}^{(f, g)}=X_{t}^{(f g)} \quad t \geq 0, \quad \text { a.s. },
$$

i.e., $X^{(f, g)}$ and $X^{(f g)}$ are indistinguishable. So $\mathscr{Z}$ is a compensated-covariation stable family of martingales.

Thanks to Theorem 4.1.9 and Proposition 4.2.3, we have that the family $\mathscr{Z}$ defined in (4.29), possesses the $\mathscr{H}^{2}$-PRP with respect to $\mathbb{F}_{+}^{\mathscr{Z}}$. From Lemma 4.2 .2 we know that $\mathbb{F}^{\mathscr{Z}}=\mathbb{F}^{L}$ hence $\mathbb{F}^{\mathscr{L}}$ is right continuous, i.e., $\mathbb{F}_{+}^{\mathscr{Z}}=\mathbb{F}^{\mathscr{Z}}$. In conclusion, we have shown that the family $\mathscr{Z}$ has the $\mathscr{H}^{2}$-PRP with respect to $\mathbb{F}^{L}$. Let us consider the family of martingales $\mathscr{X}$ defined in (3.1), i.e.,

$$
\mathscr{X}:=\left\{\mathrm{W}^{\sigma}\right\} \cup\left\{X^{(f)}, \quad f \in L^{2}(\nu)\right\} .
$$

Obviously, $\mathscr{Z} \subseteq \mathscr{X} \cup\{0\}$ and so $\mathscr{L}^{2}(\mathscr{Z}) \subseteq \mathscr{L}^{2}(\mathscr{X})$. On the other side, $\mathscr{L}^{2}(\mathscr{Z})=$ $\mathscr{H}_{0}^{2}$ and $\mathscr{L}^{2}(\mathscr{X}) \subseteq \mathscr{H}_{0}^{2}$. We can then claim that the family $\mathscr{X}$ has the $\mathscr{H}^{2}$ PRP with respect to $\mathbb{F}^{L}$. We summarize the results obtained until now in the next theorem. 
4.2.4 Theorem. The family $\mathscr{X}$ introduced in (3.1) possesses the $\mathscr{H}^{2}-P R P$ with respect to $\mathbb{F}^{L}$.

Although Theorem 4.2.4 is an interesting theoretical result, the family $\mathscr{X}$ seems to be too large for applications. In particular, it is always uncountable. For any arbitrary system of functions in $L^{2}(\nu)$, say $\mathscr{T}$, we define the family $\mathscr{X}_{\mathscr{T}}$ by

$$
\mathscr{X}_{\mathscr{T}}:=\left\{\mathrm{W}^{\sigma}\right\} \cup\left\{X^{(f)}, \quad f \in \mathscr{T}\right\} .
$$

Our aim is to establish necessary and sufficient conditions on the system $\mathscr{T} \subseteq L^{2}(\nu)$ for the family $\mathscr{X}_{\mathscr{T}}$ to have the $\mathscr{H}^{2}$-PRP with respect to $\mathbb{F}^{L}$. In other words, we want to reduce the family $\mathscr{X}$ as much as possible without losing the $\mathscr{H}^{2}$-PRP. The following theorem characterizes the $\mathscr{H}^{2}$-PRP of the family $\mathscr{X}_{\mathscr{T}}$ in terms of the system $\mathscr{T}$.

4.2.5 Theorem. Let $\mathscr{T}$ be a system of functions in $L^{2}(\nu)$. The family $\mathscr{X}_{\mathscr{T}}$ defined in (4.32) possesses the $\mathscr{H}^{2}-P R P$ with respect to $\mathbb{F}^{L}$ if and only if $\mathscr{T}$ is a total system in $L^{2}(\nu)$.

Proof. First we assume that the family $\mathscr{X}_{\mathscr{T}}$ possesses the $\mathscr{H}^{2}$-PRP and show that $\mathscr{T}$ is total in $L^{2}(\nu)$. If $\mathscr{X}_{\mathscr{T}}$ has the $\mathscr{H}^{2}$-PRP, by Theorem 1.4.11.(ii) it satisfies condition $C_{2}$. We consider a function $h \in L^{2}(\nu)$ which is orthogonal to the closure in $L^{2}(\nu)$ of $\operatorname{Span}(\mathscr{T})$. By Theorem 2.2.16.(vi), the martingale $X^{(h)} \in \mathscr{H}_{\mathrm{loc}, 0}^{2}$ associated with $h$ is orthogonal to $\mathscr{X}_{\mathscr{T}}$. But then, by condition $C_{2}$, we have $X^{(h)}=0$ and again by Theorem 2.2.16.(v), $h=0$. Therefore $\mathscr{T}$ is total in $L^{2}(\nu)$. Conversely, we assume that $\mathscr{T}$ is total in $L^{2}(\nu)$ and we show that $\mathscr{X}_{\mathscr{T}}$ has the $\mathscr{H}^{2}$-PRP with respect to $\mathbb{F}^{L}$. For any deterministic time $N \in \mathbb{N}$ we introduce the families

$$
\mathscr{X}^{N}:=\left\{\mathrm{W}^{\sigma N}\right\} \cup\left\{X^{(f)^{N}}, \quad f \in L^{2}(\nu)\right\} ; \quad \mathscr{X}_{\mathscr{T}}^{N}:=\left\{\mathrm{W}^{\sigma N}\right\} \cup\left\{X^{(f)^{N}}, \quad f \in \mathscr{T}\right\} .
$$

Under the assumption that $\mathscr{T}$ is total in $L^{2}(\nu)$, we know that for every $f \in L^{2}(\nu)$ there exists a sequence $\left(f_{n}\right)_{n \in \mathbb{N}} \subseteq \operatorname{Span}(\mathscr{T})$ such that $f_{n} \longrightarrow f$ in $L^{2}(\nu)$ as $n \rightarrow+\infty$. Because of the linearity of $X^{(g)}$ with respect to $g \in L^{2}(\nu)$, we can conclude that $X^{\left(f_{n}\right)} \in \operatorname{Span}\left(\mathscr{X}_{\mathscr{T}}\right)$. Now we fix $N \in \mathbb{N}$. By isometry we get

$$
\left\|X_{N}^{(f)}-X_{N}^{\left(f_{n}\right)}\right\|_{L^{2}(\mathbb{P})}=\left\|X_{N}^{\left(f-f_{n}\right)}\right\|_{L^{2}(\mathbb{P})}=\sqrt{N}\left\|f-f_{n}\right\|_{L^{2}(\nu)} \longrightarrow 0, \quad n \rightarrow+\infty .
$$

The processes $X^{(f)^{N}}$ and $X^{\left(f_{n}\right)^{N}}$ belong to $\mathscr{H}_{0}^{2}$ for every $N \in \mathbb{N}$ and, by Doob's inequality, we get that $X^{\left(f_{n}\right)^{N}} \longrightarrow X^{(f)^{N}}$ in $\left(\mathscr{H}_{0}^{2},\|\cdot\|_{\mathscr{H}_{0}^{2}}\right)$ as $n \rightarrow+\infty$. This means

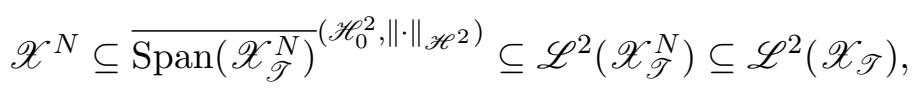

the last inclusion being true because $\mathscr{L}^{2}\left(\mathscr{X}_{\mathscr{T}}\right)$ is a stable subspace of $\mathscr{H}_{0}^{2}$ and $\mathscr{X}_{\mathscr{T}} \subseteq \mathscr{H}_{\mathrm{loc}, 0}^{2}$, so that $\mathscr{X}_{\mathscr{T}}^{N} \subseteq \mathscr{L}^{2}\left(\mathscr{X}_{\mathscr{T}}\right)$ (cf. Lemma 1.4.4). By Lemma 3.4.1, the following identities hold

$$
\mathscr{L}^{2}\left(\left\{\mathrm{~W}^{\sigma N}, N \in \mathbb{N}\right\}\right)=\mathscr{L}^{2}\left(\mathrm{~W}^{\sigma}\right) ; \quad \mathscr{L}^{2}\left(\left\{X^{(f)^{N}}, N \in \mathbb{N}\right\}\right)=\mathscr{L}^{2}\left(X^{(f)}\right), \quad f \in \mathscr{T} .
$$


Consequently,

$$
\mathscr{L}^{2}\left(\mathrm{~W}^{\sigma}\right) \cup \bigcup_{f \in L^{2}(\nu)} \mathscr{L}^{2}\left(X^{(f)}\right) \subseteq \mathscr{L}^{2}\left(\mathscr{X}_{\mathscr{T}}\right)
$$

From Theorem 1.4.5.(ii), the left-hand side in the previous relation is total in $\mathscr{L}^{2}(\mathscr{X})$ and, since $\mathscr{L}^{2}\left(\mathscr{X}_{\mathscr{T}}\right)$ is linear and closed in $\left(\mathscr{H}_{0}^{2},\|\cdot\|_{\mathscr{H}_{0}^{2}}\right)$, we conclude

$$
\mathscr{L}^{2}(\mathscr{X}) \subseteq \mathscr{L}^{2}\left(\mathscr{X}_{\mathscr{T}}\right) \subseteq \mathscr{H}_{0}^{2} .
$$

Theorem 4.2.4 yields $\mathscr{L}^{2}(\mathscr{X})=\mathscr{H}_{0}^{2}$ and hence $\mathscr{L}^{2}\left(\mathscr{X}_{\mathscr{T}}\right)=\mathscr{H}_{0}^{2}$, meaning that $\mathscr{X}_{\mathscr{T}}$ possesses the $\mathscr{H}^{2}$-PRP with respect to $\mathbb{F}^{L}$.

Notice that with Theorem 4.2.5, we gave a characterization of the $\mathscr{H}^{2}$-PRP of a system $\mathscr{X}_{\mathscr{T}}$ in terms of the totality in $L^{2}(\nu)$ of the system $\mathscr{T}$. This was possible in the general case, without further restriction on the system $\mathscr{T}$. We could not obtain a similar result for the $\mathscr{H}^{1}$-PRP. In Theorem 3.4 .2 we only proved that if a system $\mathscr{T} \subseteq L^{2}(\nu)$ is total in $L^{2}(\nu)$, then the associated family of martingales possesses the $\mathscr{H}^{1}$-PRP. Only in the special case in which $\mathscr{T} \subseteq L^{2}(\nu)$ is a finite system, we could show that the totality of $\mathscr{T}$ is necessary for $\mathscr{X}_{\mathscr{T}}$ to have the $\mathscr{H}^{1}$-PRP (cf. Proposition 3.4.5).

A first consequence of Theorem 4.2.5 is the possibility of finding countable systems of martingales with the $\mathscr{H}^{2}$-PRP.

4.2.6 Proposition. It is always possible to find a countable family of martingales with the $\mathscr{H}^{2}-P R P$ with respect to $\mathbb{F}^{L}$.

Proof. The space $L^{2}(\nu)$ is a Hilbert space on the measurable space $(\mathbb{R}, \mathscr{B}(\mathbb{R}))$. Because $\mathscr{B}(\mathbb{R})$ is a separable $\sigma$-algebra, $L^{2}(\nu)$ is a separable Hilbert space and so there always exists a system $\mathscr{T}$ which is countable and total in $L^{2}(\nu)$. From Theorem 4.2.5, the associated family $\mathscr{X}_{\mathscr{T}}$ is a countable family of martingales with the $\mathscr{H}^{2}$ PRP.

We conclude this section turning to the problem of finding finite families of martingales with the $\mathscr{H}^{2}$-PRP. A similar problem was considered for the $\mathscr{H}^{1}$-PRP. We shall obtain similar conclusions as in Corollary 3.4.6: Although the possibility of finding families of martingales $\mathscr{X}_{\mathscr{T}}$ consisting of finitely many elements and possessing the $\mathscr{H}^{2}-\mathrm{PRP}$ is of special interest, this is the case in a rather limited generality.

4.2.7 Corollary. The following statements are equivalent:

(i) There exists a finite family $\mathscr{X}_{\mathscr{T}}$ possessing the $\mathscr{H}^{2}-P R P$ with respect to $\mathbb{F}^{L}$.

(ii) $L^{2}(\nu)$ is finite-dimensional.

(iii) $\nu$ has finite support.

Proof. The equivalence of (ii) and (iii) is obvious. The equivalence of (i) and (ii) is an immediate consequence of Theorem 4.2.5. Indeed, the family $\mathscr{X}_{\mathscr{T}}$ has the $\mathscr{H}^{2}$ PRP with respect to $\mathbb{F}^{L}$ if and only if $\mathscr{T}$ is total. But $\mathscr{X}_{\mathscr{T}}$ is finite if and only if $\mathscr{T}$ is finite. Therefore there exists a finite family $\mathscr{X}_{\mathscr{T}}$ with the $\mathscr{H}^{2}$-PRP if and only if there exists a finite total system in $L^{2}(\nu)$, which is equivalent to say that $L^{2}(\nu)$ is finite dimensional.

The situation discussed in Corollary 4.2 .7 occurs, for example, if $\left(L, \mathbb{F}^{L}\right)$ is a simple Lévy process (cf. (3.10)) 


\subsubsection{Complete Orthogonal Systems}

We now come to the important case in which the system $\mathscr{T} \subseteq L^{2}(\nu)$ is a complete orthogonal system, i.e., a total system consisting of orthogonal functions. Note that a complete orthogonal system of a Hilbert space is always countable and so we use the notation $\mathscr{T}:=\left\{f_{n}, n \geq 1\right\}$. In this situation the $\mathscr{H}^{2}$-PRP becomes particularly interesting, as the next theorem shows.

4.2.8 Theorem. Let $\mathscr{T}$ be a complete orthogonal system in $L^{2}(\nu)$. The associated family $\mathscr{X}_{\mathscr{T}}$ has the $\mathscr{H}^{2}-P R P$ and any martingale $M$ belonging to $\mathscr{H}_{0}^{2}$ can be represented as

$$
M=\phi \cdot \mathrm{W}^{\sigma}+\sum_{n=1}^{\infty} H^{n} \cdot X^{\left(f_{n}\right)}, \quad \phi \in \mathrm{L}^{2}\left(\mathrm{~W}^{\sigma}\right), \quad H^{n} \in \mathrm{L}^{2}\left(X^{\left(f_{n}\right)}\right), \quad n \geq 1,
$$

where the series converges in $\left(\mathscr{H}_{0}^{2},\|\cdot\|_{\mathscr{H}^{2}}\right)$.

Proof. Because of the orthogonality of $\mathscr{T}$, the associated family of martingales $\mathscr{X}_{\mathscr{T}}$ is orthogonal (cf. Theorem 2.2.16.(vi)). Thanks to Theorem 4.2.5 we have

$$
\mathscr{L}^{2}\left(\mathscr{X}_{\mathscr{T}}\right)=\mathscr{H}_{0}^{2} .
$$

By Theorem 1.4.8, the orthogonality of $\mathscr{X}_{\mathscr{T}}$ implies that the generated stable subspace $\mathscr{L}^{2}\left(\mathscr{X}_{\mathscr{T}}\right)$ has the following structure:

$$
\mathscr{L}^{2}\left(\mathscr{X}_{\mathscr{T}}\right)=\mathscr{L}^{2}\left(\mathrm{~W}^{\sigma}\right) \oplus \bigoplus_{n=1}^{\infty} \mathscr{L}^{2}\left(X^{\left(f_{n}\right)}\right)
$$

Combining the previous two identities we get (4.33).

If $\mathscr{T} \subseteq L^{2}(\nu)$ is a complete orthogonal system, we can give a characterization of the spaces $\mathscr{H}_{0}^{2, d}$ and $\mathscr{H}_{0}^{2, c}$ of the purely discontinuous and continuous martingales in $\mathscr{H}_{0}^{2}$, respectively.

4.2.9 Corollary. Let $\left(L, \mathbb{F}^{L}\right)$ be a Lévy process with characteristic triplet $\left(\beta, \sigma^{2}, \nu\right)$ and let $\mathscr{T} \subseteq L^{2}(\nu)$ be a complete orthogonal system. We consider the family of martingales $\mathscr{X}_{\mathscr{T}}:=\left\{\mathrm{W}^{\sigma}\right\} \cup\left\{X^{\left(f_{n}\right)}, \quad n \geq 1\right\}$ associated with $\mathscr{T}$, where $\mathrm{W}^{\sigma}$ is the Gaussian part of L. A martingale $M \in \mathscr{H}_{0}^{2}\left(\mathbb{F}^{L}\right)$ belongs to $\mathscr{H}_{0}^{2, d}$ if and only if

$$
M=\sum_{i=1}^{\infty} H^{n} \cdot X^{\left(f_{n}\right)}, \quad H^{n} \in \mathrm{L}^{2}\left(X^{\left(f_{n}\right)}\right), \quad n \geq 1,
$$

while $M$ belongs to $\mathscr{H}_{0}^{2, c}$ if and only if

$$
M=\phi \cdot \mathrm{W}^{\sigma}, \quad \phi \in \mathrm{L}^{2}\left(\mathrm{~W}^{\sigma}\right) .
$$

Proof. Because of Theorem 4.2.8, if $M \in \mathscr{H}_{0}^{2}\left(\mathbb{F}^{L}\right)$, we can write

$$
M=\phi \cdot \mathrm{W}^{\sigma}+\sum_{n=1}^{\infty} H^{n} \cdot X^{\left(f_{n}\right)}, \quad \phi \in \mathrm{L}^{2}\left(\mathrm{~W}^{\sigma}\right), \quad H^{n} \in \mathrm{L}^{2}\left(X^{\left(f_{n}\right)}\right), \quad n \geq 1 .
$$


Each summand of the series on the right-hands side of the previous formula belongs to $\mathscr{H}_{0}^{2, d}$ because $\mathscr{H}_{0}^{2, d}$ is a stable subspace of $\mathscr{H}_{0}^{2}\left(\mathbb{F}^{L}\right)$ (cf. Proposition 1.4.2). Moreover, the series converges in $\left(\mathscr{H}_{0}^{2},\|\cdot\|_{\mathscr{H}^{2}}\right)$. From Jacod (1979), Lemma 2.12, the space $\mathscr{H}_{0}^{2, d}$ is closed in $\left(\mathscr{H}_{0}^{2},\|\cdot\|_{\mathscr{H}^{2}}\right)$, therefore there exists $N \in \mathscr{H}_{0}^{2, d}$ such that

$$
N=\sum_{n=1}^{\infty} H^{n} \cdot X^{\left(f_{n}\right)}, \quad H^{n} \in \mathrm{L}^{2}\left(X^{\left(f_{n}\right)}\right), \quad n \geq 1 .
$$

The martingale $\phi \cdot \mathrm{W}^{\sigma}, \phi \in \mathrm{L}^{2}\left(\mathrm{~W}^{\sigma}\right)$, belongs to $\mathscr{H}_{0}^{2, c}$. In conclusion, we have that $M \in \mathscr{H}_{0}^{2, d}$ if and only the term $\phi \cdot \mathrm{W}^{\sigma}$ in (4.33) vanishes and so, if an d only if $M=N$. Conversely, $M \in \mathscr{H}_{0}^{2, c}$ if and only if $N$ vanishes and so, if and only if $M=\phi \cdot \mathrm{W}^{\sigma}$.

Let $\mathscr{T} \subseteq L^{2}(\nu)$ be a complete orthogonal system. Then the associated family of martingales $\mathscr{X}_{\mathscr{T}}$ possesses the $\mathscr{H}^{2}$-PRP and formula (4.33) holds. An interesting question is whether the integrands appearing in the stochastic integrals in (4.33) are uniquely determined by the martingale $M \in \mathscr{H}_{0}^{2}$.

4.2.10 Theorem. Let $\mathscr{T}$ be a complete orthogonal system in $L^{2}(\nu)$ and $\mathscr{X}_{\mathscr{T}}$ the associated family of martingales. Any $Z \in \mathscr{H}_{0}^{2}$ can be represented as in (4.33) and the integrands are $\lambda_{+} \otimes \mathbb{P}$-a.e. uniquely determined by $Z$.

Proof. Let $Z \in \mathscr{H}_{0}^{2}$ have two representations of the form (4.33), i.e.,

$$
Z=\phi \cdot \mathrm{W}^{\sigma}+\sum_{n=1}^{\infty} H^{n} \cdot X^{\left(f_{n}\right)}=\psi \cdot \mathrm{W}^{\sigma}+\sum_{n=1}^{\infty} K^{n} \cdot X^{\left(f_{n}\right)}
$$

for $\phi, \psi \in \mathrm{L}^{2}\left(\mathrm{~W}^{\sigma}\right), K^{n}, H^{n} \in \mathrm{L}^{2}\left(X^{\left(f_{n}\right)}\right), n \geq 1$. Then

$$
(\phi-\psi) \cdot \mathrm{W}^{\sigma}+\sum_{n=1}^{\infty}\left(H^{n}-K^{n}\right) \cdot X^{\left(f_{n}\right)}=0 .
$$

Now we fix $k \geq 1$ and consider the martingale $X^{\left(f_{k}\right)}$. If $J \in \mathbb{N}$ is a deterministic stopping time, we put

$$
N^{J}:=X^{\left(f_{k}\right)^{J}}
$$

and $N^{J} \in \mathscr{H}_{0}^{2}$ (cf. Theorem 2.2.16.(iii)). If $n \neq k$, then $X^{\left(f_{k}\right)}$ is orthogonal to $X^{\left(f_{n}\right)}$. By Proposition 1.2.10.(iii), the same holds for $N^{J}$. We note that $\left[N^{J}, N^{J}\right]$ is integrable because $N^{J} \in \mathscr{H}_{0}^{2}$ (cf. Proposition 1.2.11.(ii)) and so $1 \in \mathrm{L}^{2}\left(N^{J}\right)$. By assumption the processes $H^{n}$ and $K^{n}$ belong to $\mathrm{L}^{2}\left(X^{\left(f_{n}\right)}\right)$. Applying Lemma 1.4.6.(i) with $M:=X^{\left(f_{n}\right)}, N:=N^{J}, H:=H^{n}-K^{n}, K:=1$ and $p=q:=2$, we get that the processes $\left(H^{n}-K^{n}\right) \cdot\left[X^{\left(f_{n}\right)}, N^{J}\right]$ belongs to $\mathscr{M}_{0}$ for $n \neq k$. If $n=k$, as a consequence of Kunita-Watanabe's inequality, the process $\left(H^{k}-K^{k}\right) \cdot\left[X^{\left(f_{k}\right)}, N^{J}\right]$ is of integrable variation. If now $T$ is a stopping time, by Lemma 1.2.2, we get

$$
\mathbb{E}\left[\left(H^{n}-K^{n}\right) \cdot\left[X^{\left(f_{n}\right)}, N^{J}\right]_{T}\right]=0, \quad n \neq k .
$$


By Lemma 1.3.1 the linear functional $\mathbb{E}\left[\left[\cdot, N^{J}\right]_{\infty}\right]$ is continuous on $\mathscr{H}_{0}^{2}$. Using this fact we can compute $\mathbb{E}\left[\left[\cdot, N^{J}\right]_{T}\right]$ in (4.36):

$$
\begin{aligned}
0 & \left.=\mathbb{E}\left[\left[\phi \cdot \mathrm{W}^{\sigma}, N^{J}\right]_{T}\right]+\sum_{n=1}^{\infty} \mathbb{E}\left[\left(H^{n}-K^{n}\right) \cdot X^{\left(f_{n}\right)}, N^{J}\right]_{T}\right] \\
& =\sum_{n=1}^{\infty} \mathbb{E}\left[\left(H^{n}-K^{n}\right) \cdot\left[X^{\left(f_{n}\right)}, N^{J}\right]_{T}\right]=\mathbb{E}\left[\left(H^{k}-K^{k}\right) \cdot\left[X^{\left(f_{k}\right)}, N^{J}\right]_{T}\right],
\end{aligned}
$$

where, in the second equality we used Proposition 1.3.4.(i) and that for every continuous local martingale $M,\left[M, N^{J}\right]=0$ because $N^{J}$ is purely discontinuous, while in the last equality we applied (4.37). Therefore the process $\left(H^{k}-K^{k}\right) \cdot\left[X^{\left(f_{k}\right)}, N^{J}\right]$ is of integrable variation and for every stopping time $T, \mathbb{E}\left[\left(H^{k}-K^{k}\right) \cdot\left[X^{\left(f_{k}\right)}, N^{J}\right]_{T}\right]=0$. Lemma 1.2.2 issues $\left(H^{k}-K^{k}\right) \cdot\left[X^{\left(f_{k}\right)}, N^{J}\right] \in \mathscr{M}_{0}$. Because $\left(H^{k}-K^{k}\right) \cdot\left[X^{\left(f_{k}\right)}, N^{J}\right]$ belongs to $\mathscr{M}_{0} \cap \mathscr{A}$, its compensator exists and is equal to zero. On the other side, $\left[X^{\left(f_{k}\right)}, N^{J}\right]$ belongs to $\mathscr{A}^{+}$and its compensator is $\left\langle X^{\left(f_{k}\right)}, N^{J}\right\rangle$ (cf. Proposition 1.2.11). Hence the compensator of $\left(H^{k}-K^{k}\right) \cdot\left[X^{\left(f_{k}\right)}, N^{J}\right]$ is $\left(H^{k}-K^{k}\right) \cdot\left\langle X^{\left(f_{k}\right)}, N^{J}\right\rangle$ (cf. Theorem 1.2.4 and the comment following it), i.e., $\left(H^{k}-K^{k}\right) \cdot\left\langle X^{\left(f_{k}\right)}, N^{J}\right\rangle=0$. Consequently,

$$
\begin{aligned}
0=\left(H^{k}-K^{k}\right) \cdot\left\langle X^{\left(f_{k}\right)}, N^{J}\right\rangle_{t} & =\left(H^{k}-K^{k}\right) \cdot\left\langle X^{\left(f_{k}\right)}, X^{\left(f_{k}\right)}{ }^{J}\right\rangle_{t} \\
& =\left(H^{k}-K^{k}\right) \cdot\left\langle X^{\left(f_{k}\right)}, X^{\left(f_{k}\right)}\right\rangle_{t}^{J} \\
& =\left(H^{k}-K^{k}\right) 1_{[0, J]} \cdot\left\langle X^{\left(f_{k}\right)}, X^{\left(f_{k}\right)}\right\rangle_{t} \\
& =\nu\left(f_{k}^{2}\right) \int_{0}^{t}\left(H_{s}^{k}-K_{s}^{k}\right) 1_{[0, J]}(s) \mathrm{d} s, \quad t \geq 0, \quad \text { a.s. }
\end{aligned}
$$

and this implies $\left(H^{k}-K^{k}\right) 1_{[0, J]}=0 \lambda_{+} \otimes \mathbb{P}$-a.e., for every $J \geq 1$. The predictable process $\left(H^{k}-K^{k}\right) 1_{[0, J]}$ converges pointwise to $\left(H^{k}-K^{k}\right)$ as $J \rightarrow+\infty$ and therefore $H^{k}-K^{k}=0 \lambda_{+} \otimes \mathbb{P}$-a.e., that is, $H^{k}=K^{k} \lambda_{+} \otimes \mathbb{P}$-a.e. Because of the arbitrariness of $k$, we can conclude that

$$
H^{k}=K^{k} \quad \lambda_{+} \otimes \mathbb{P} \text {-a.e., } \quad k \geq 1 .
$$

By (4.36), this implies that $(\psi-\phi) \cdot \mathrm{W}^{\sigma}=0$ which yields

$$
0=(\psi-\phi)^{2} \cdot\left\langle\mathrm{W}^{\sigma}, \mathrm{W}^{\sigma}\right\rangle_{t}=\sigma^{2} \int_{0}^{t}\left(\psi_{s}-\phi_{s}\right)^{2} \mathrm{~d} s, \quad t \geq 0, \quad \text { a.s. }
$$

and therefore $\psi=\phi \lambda_{+} \otimes \mathbb{P}$-a.e. This concludes the proof.

Complete orthogonal systems in $L^{2}(\nu)$ are related with minimal families of martingales with the $\mathscr{H}^{2}$-PRP.

4.2.11 Definition. Let $q \geq 1$ and $\mathscr{Z}$ be a family of local martingales with the $\mathscr{H}^{q}$-PRP. We say that $\mathscr{Z}$ is a minimal family in $\mathscr{H}_{0}^{q}$ if no element of $\mathscr{Z}$ can be omitted without loosing the $\mathscr{H}^{q}$-PRP. 
If $\mathscr{T}$ is a total system in $L^{2}(\nu)$, the associated family $\mathscr{X}_{\mathscr{T}}$ need not to be a minimal family in $\mathscr{H}_{0}^{2}$. Indeed, as we shall see, it can happen that it is possible to omit some elements from the system $\mathscr{T}$ and nevertheless it remains total. However, if $\mathscr{T}$ is a complete orthogonal system, then the family $\mathscr{X}_{\mathscr{T}}$ is a minimal family in $\mathscr{H}_{0}^{2}$ as stated in the following proposition.

4.2.12 Proposition. Let $\mathscr{T}$ be a complete orthogonal system in $L^{2}(\nu)$. The associated family of martingales $\mathscr{X}_{\mathscr{T}}$ is a minimal family in $\mathscr{H}_{0}^{2}$.

Proof. To omit a martingale from the family $\mathscr{X}_{\mathscr{T}}$ means either to omit the Gaussian part $\mathrm{W}^{\sigma}$ or a function $h \in \mathscr{T}$. The Gaussian part $\mathrm{W}^{\sigma}$ can clearly not be omitted without loosing the $\mathscr{H}^{2}$-PRP. Indeed, by means of the family $\left\{X^{(f)}, f \in \mathscr{T}\right\}$ we cannot represent continuous martingales in $\mathscr{H}_{0}^{2}\left(\mathbb{F}^{L}\right)$, because the space $\mathscr{H}_{0}^{2, d}$ is a stable subspaces of $\mathscr{H}_{0}^{2}$ (cf. Corollary 4.2.9). If we omit a function $h$ from $\mathscr{T}$, we loose the totality of $\mathscr{T}$ in $L^{2}(\nu)$ and from Theorem 4.2.5, $\mathscr{X}_{\mathscr{T} \backslash\{h\}}$ cannot have the $\mathscr{H}^{2}$-PRP.

Although the problem of finding a family $\mathscr{X}_{\mathscr{T}}$ with the $\mathscr{H}^{1}$-PRP which is moreover minimal in $\mathscr{H}_{0}^{1}$ is interesting, we were not able to furnish a positive answer to this question.

\subsubsection{Square Integrable Lévy Processes}

This part is analogous to $\S 3.5$ but we shall follow a different approach. In Theorem 4.2.5, given a Lévy process $\left(L, \mathbb{F}^{L}\right)$ with Lévy measure $\nu$, we showed that if $\mathscr{T} \subseteq$ $L^{2}(\nu)$ is a total system, then the family $\mathscr{X}_{\mathscr{T}}$ defined by

$$
\mathscr{X}_{\mathscr{T}}:=\left\{\mathrm{W}^{\sigma}\right\} \cup\left\{X^{(f)}, \quad f \in \mathscr{T}\right\}
$$

possesses the $\mathscr{H}^{2}$-PRP with respect to $\mathbb{F}^{L}$ and conversely, if $\mathscr{X}_{\mathscr{T}}$ has the $\mathscr{H}^{2}$-PRP, then $\mathscr{T}$ is total in $L^{2}(\nu)$. To prove this result we used that the bigger family

$$
\mathscr{X}:=\left\{\mathrm{W}^{\sigma}\right\} \cup\left\{X^{(f)}, \quad f \in L^{2}(\nu)\right\}
$$

possesses the $\mathscr{H}^{2}$-PRP with respect to $\mathbb{F}^{L}$ (cf. Theorem 4.2.4). Although both $\mathscr{X}$ and $\mathscr{X}_{\mathscr{T}}$ were constructed starting from the structure of the Lévy process, $L$ does not appear in them explicitly. Here we show that, under further regularity assumptions on the Lévy process $L$, we can replace the Gaussian part $\mathrm{W}^{\sigma}$ by $L$ itself. Thereafter, we assume that $L$ is square integrable, i.e., that $L_{t} \in L^{2}(\mathbb{P})$ for every $t \geq 0$. According to Theorem 2.3.8.(ii) this is equivalent to require that the function $1_{\{|x|>1\}} x$ belongs to $L^{2}(\nu)$ and this implies that $x \in L^{2}(\nu)$. For a square integrable Lévy process $\left(L, \mathbb{F}^{L}\right)$ we define the process $\bar{L}:=\left(\bar{L}_{t}\right)_{t \geq 0}$ by

$$
\bar{L}_{t}:=L_{t}-\mathbb{E}\left[L_{t}\right], \quad t \geq 0,
$$

which is an $\mathbb{F}^{L}$-martingale because of Lemma 2.1.1. By $\overline{\mathscr{X}}$ we designate the following family of martingales in $\mathscr{H}_{\text {loc }, 0}^{2}$ :

$$
\overline{\mathscr{X}}:=\{\bar{L}\} \cup\left\{X^{(f)}, \quad f \in L^{2}(\nu)\right\} .
$$


Let $\mathscr{T} \subseteq L^{2}(\nu)$. We put

$$
\overline{\mathscr{X}}_{\mathscr{T}}:=\{\bar{L}\} \cup\left\{X^{(f)}, \quad f \in \mathscr{T}\right\} .
$$

We now show that the family $\overline{\mathscr{X}}$ possesses the $\mathscr{H}^{2}$-PRP.

4.2.13 Theorem. Let $\left(L, \mathbb{F}^{L}\right)$ be a square integrable Lévy process. The family $\overline{\mathscr{X}}$ defined in (4.39) possesses the $\mathscr{H}^{2}-P R P$ with respect to $\mathbb{F}^{L}$

Proof. In $\S 3.5$, we showed that $\overline{\mathscr{X}}$ has the $\mathscr{H}^{1}$-PRP with respect to $\mathbb{F}^{L}$, by showing the uniqueness of the solution of an appropriate martingale problem associated with $\overline{\mathscr{X}}$. Now we prove that the stable subspace generated by $\overline{\mathscr{X}}$ in $\mathscr{H}_{0}^{2}, \mathscr{L}^{2}(\overline{\mathscr{X}})$, is equal to $\mathscr{L}^{2}(\mathscr{X})$. By Theorem 4.2.4 we have $\mathscr{L}^{2}(\mathscr{X})=\mathscr{H}_{0}^{2}$, therefore $\mathscr{L}^{2}(\overline{\mathscr{X}}) \subseteq$ $\mathscr{L}^{2}(\mathscr{X})$. To show the other inclusion it is enough to verify that $\mathscr{L}^{2}\left(\mathrm{~W}^{\sigma}\right)$ is contained in $\mathscr{L}^{2}(\overline{\mathscr{X}})$. From the Itô-Lévy decomposition of $L$ and Lemma 2.2.9, it follows that $\mathbb{E}\left[L_{t}\right]=t\left(\beta+\nu\left(1_{\{|x|>1\}} x\right)\right)$. Moreover, by the square integrability of $L$, we have $x \in L^{2}(\nu)$, so that we can define the martingale $X^{(x)} \in \mathscr{H}_{\mathrm{loc}, 0}^{2}$ and we can rewrite the Itô-Lévy decomposition of $L$ as it follows: $\bar{L}=\mathrm{W}^{\sigma}+X^{(x)}$. Hence $\mathrm{W}^{\sigma}:=\bar{L}-X^{(x)}$. The processes $\bar{L}$ and $X^{(x)}$ belong to $\mathscr{H}_{\mathrm{loc}, 0}^{2}$ and for any deterministic time $N \in \mathbb{N}$, $\bar{L}^{N}$ and $X^{(x)^{N}}$ belong to $\mathscr{H}_{0}^{2}$ : For $X^{(x)}$ this follows from Theorem 2.2.16.(iii), while for $\bar{L}$ we have

$$
\sup _{t \geq 0} \mathbb{E}\left[\left(\bar{L}_{t}^{N}\right)^{2}\right]=\mathbb{E}\left[\bar{L}_{N}^{2}\right]<+\infty
$$

and Doob's inequality yields $\sup _{t \geq 0}\left(\bar{L}_{t}^{N}\right)^{2} \in L^{1}(\mathbb{P})$. By Lemma 1.4.4, $\bar{L}^{N}$ and $X^{(x)^{N}}$ belong to $\mathscr{L}^{2}(\overline{\mathscr{X}})$, for every $N \in \mathbb{N}$. Therefore $\mathrm{W}^{\sigma N}$ belongs to $\mathscr{L}^{2}(\overline{\mathscr{X}})$ for every $N$. The stable subspace $\mathscr{L}^{2}\left(\left\{\mathrm{~W}^{\sigma N}, N \in \mathbb{N}\right\}\right)$ is contained in $\mathscr{L}^{2}(\overline{\mathscr{X}})$, but, by Lemma 3.4.1, $\mathscr{L}^{2}\left(\left\{\mathrm{~W}^{\sigma N}, N \in \mathbb{N}\right\}\right)=\mathscr{L}^{2}\left(\mathrm{~W}^{\sigma}\right)$ and the proof is finished.

We are going to establish the analogue of Theorem 4.2 .5 for $\overline{\mathscr{X}}_{\mathscr{T}}$. Now, to show that if $\overline{\mathscr{X}}_{\mathscr{T}}$ has the $\mathscr{H}^{2}$-PRP, then the system $\mathscr{T}$ is total is $L^{2}(\nu)$, we need to proceed in a slightly different way. Indeed, if we consider a function $h$ which is orthogonal to $\mathscr{T}$, the martingale $X^{(h)}$ need not to be orthogonal to $\overline{\mathscr{X}}_{\mathscr{T}}$ because it could happen that $X^{(h)}$ is not orthogonal to the martingale $\bar{L}=\mathrm{W}^{\sigma}+X^{(x)}$.

4.2.14 Theorem. Let $\left(L, \mathbb{F}^{L}\right)$ be a square integrable Lévy process. The family $\overline{\mathscr{X}}_{\mathscr{T}}$ has the $\mathscr{H}^{2}-P R P$ and the function $x$ belongs to the closure in $L^{2}(\nu)$ of $\operatorname{Span}(\mathscr{T})$ if and only if $\mathscr{T}$ is total in $L^{2}(\nu)$.

Proof. Obviously, if $\mathscr{T}$ is total in $L^{2}(\nu)$ we have that $x$ belongs to the closure in $L^{2}(\nu)$ of $\operatorname{Span}(\mathscr{T})$ and the proof that $\overline{\mathscr{X}}_{\mathscr{T}}$ has the $\mathscr{H}^{2}$-PRP is similar to the proof that $\mathscr{X}_{\mathscr{T}}$ has the $\mathscr{H}^{2}$-PRP (cf. Theorem 4.2.5). Let us assume that $\overline{\mathscr{X}}_{\mathscr{T}}$ has the $\mathscr{H}^{2}$-PRP and that $x$ belongs to the closure in $L^{2}(\nu)$ of $\operatorname{Span}(\mathscr{T})$. If $h \in L^{2}(\nu)$ is orthogonal to the closure in $L^{2}(\nu)$ of $\operatorname{Span}(\mathscr{T})$, then, from $\bar{L}=\mathrm{W}^{\sigma}+X^{(x)}$, the martingale $X^{(h)}$ is orthogonal to $\overline{\mathscr{X}}_{\mathscr{T}}$ and, because $\overline{\mathscr{X}}_{\mathscr{T}}$ satisfies condition $C_{2}$, this implies that $X^{(h)}=0$ and so $h=0$. In particular, we can assert that $\mathscr{T} \subseteq L^{2}(\nu)$ is total in $L^{2}(\nu)$.

Now we deduce an analogue to Corollary 4.2.7. 
4.2.15 Corollary. The following statements are equivalent:

(i) There exists a finite family $\overline{\mathscr{X}}_{\mathscr{T}}$ possessing the $\mathscr{H}^{2}$-PRP with respect to $\mathbb{F}^{L}$.

(ii) $L^{2}(\nu)$ is finite-dimensional.

(iii) $\nu$ has finite support.

Proof. The equivalence of (ii) and (iii) is obvious. If $L^{2}(\nu)$ is finite dimensional there exists a system $\mathscr{T}$ which is finite and total. From Theorem 4.2 .14 we get that $\overline{\mathscr{X}}_{\mathscr{T}}$ is a finite family with the $\mathscr{H}^{2}$-PRP and so (ii) implies (i). For the converse implication we recall that $x \in L^{2}(\nu)$ because $L$ is square integrable. If the finite family $\overline{\mathscr{X}}_{\mathscr{T}}$ has the $\mathscr{H}^{2}$-PRP, then the family $\overline{\mathscr{X}}_{\mathscr{T}^{x}}$ has the $\mathscr{H}^{2}$-PRP, where $\mathscr{T}^{x}:=\mathscr{T} \cup\{x\}$. Therefore, by Theorem 4.2.14, $\mathscr{T}^{x}$ must be total in $L^{2}(\nu)$ and so $L^{2}(\nu)$ is finite dimensional because $\mathscr{T}^{x}$ is a finite total system.

Note that now to an orthogonal system $\mathscr{T} \subseteq L^{2}(\nu)$ there corresponds a family of orthogonal martingales $\overline{\mathscr{X}}_{\mathscr{T}}$ if and only if $x$ is orthogonal to $\mathscr{T}$. This means that if $\mathscr{T}$ is a complete orthogonal system in $L^{2}(\nu)$, the associated family $\overline{\mathscr{X}}_{\mathscr{T}}$ cannot be an orthogonal family. Indeed, if it were the case, we would have that $\mathscr{T}$ is a total system such that $x \perp \mathscr{T}$, which is impossible. However, if $\mathscr{T}$ is a countable total family, we can obtain a family of orthogonal martingales possessing the $\mathscr{H}^{2}$-PRP directly by orthogonalizing $\overline{\mathscr{X}}_{\mathscr{T}}$. We refer to, e.g., Kunita \& Watanabe (1967) for an analogue of the Gram-Schmidt orthogonalization procedure for locally square integrable martingales. We denote by $\overline{\mathscr{X}}_{\mathscr{T}}^{\text {orth }}$ such an orthogonalization. Then any martingale $M \in \mathscr{H}_{0}^{2}$ can be represented as an infinite sum of stochastic integrals with respect to elements of $\overline{\mathscr{X}}_{\mathscr{T}}^{\text {orth }}$, i.e.,

$$
M=\sum_{n=1}^{n} H^{n} \cdot M^{n}, \quad H^{n} \in \mathrm{L}^{2}\left(M^{n}\right), \quad M^{n} \in \overline{\mathscr{X}}_{\mathscr{T}}^{\text {orth }}, \quad n \geq 1,
$$

and the family $\overline{\mathscr{X}}_{\mathscr{T}}^{\text {orth }}$ is minimal in $\mathscr{H}_{0}^{2}$. Moreover, the integrands appearing in the previous decomposition are $\lambda_{+} \otimes \mathbb{P}$-a.e. uniquely determined by $M$. To prove this result it is enough to adapt the proof of Theorem 4.2 .10 to the family $\overline{\mathscr{X}}_{\mathscr{T}}^{\text {orth }}$. 
CHAPTER 5

Examples

In this chapter, given a Lévy process $\left(L, \mathbb{F}^{L}\right)$ on the probability space $\left(\Omega, \mathscr{F}_{\infty}^{L}, \mathbb{P}\right)$, we want to apply the theory developed in Chapter 4 to construct some concrete families of martingales possessing the $\mathscr{H}^{2}$-PRP with respect to $\mathbb{F}^{L}$. Let $\left(\beta, \sigma^{2}, \nu\right)$ be the characteristic triplet of $L$. Thanks to Theorem 4.2.5 every total system $\mathscr{T} \subseteq L^{2}(\nu)$ leads to a family of locally square integrable martingales with the $\mathscr{H}^{2}$-PRP with respect to $\mathbb{F}^{L}$. Of course the more regular the Lévy measure $\nu$ is, the better are the properties which we can require on the system $\mathscr{T}$. For example we shall see that, under rather strong assumptions on the measure $\nu$ we can choose $\mathscr{T} \subseteq L^{2}(\nu)$ as the system of monomials in $x$. We stress that this choice is not always possible because monomials are, in general, not contained in $L^{2}(\nu)$. We shall see that the problem of finding families of locally square integrable martingales with the $\mathscr{H}^{2}$-PRP with respect to $\mathbb{F}^{L}$ can also be solved in an interesting way also for more general Lévy measures.

For reasons of shortness, we consider in this chapter only the $\mathscr{H}^{2}$-PRP, but all the systems which we shall meet possess also the $\mathscr{H}^{1}$-PRP. However, in the first case we can obtain better results than in the latter one. Indeed, thanks to Theorem 4.2.8, complete orthogonal systems in $L^{2}(\nu)$ play a special role for the $\mathscr{H}^{2}$-PRP because the structure of the stable subspaces generated in $\mathscr{H}_{0}^{2}$ by the associated families of martingales becomes particularly simple.

\subsection{Compensated Poisson Processes}

The first example that we discuss is elementary but important because it exhibits a concrete family of locally square integrable martingales with the $\mathscr{H}^{2}$-PRP that can be introduced for any Lévy process $L$, without further assumptions on the Lévy measure $\nu$. Let $\left(L, \mathbb{F}^{L}\right)$ be a Lévy process with characteristic triplet $\left(\beta, \sigma^{2}, \nu\right)$. We define the system $\tilde{\mathscr{T}}_{D} \subseteq L^{2}(\nu)$ by

$$
\tilde{\mathscr{T}}_{D}:=\left\{1_{(a, b]}, \quad 0 \notin[a, b], \quad a, b \in \tilde{D}\right\},
$$


where $D \subseteq \mathbb{R}$ is a countable dense set as, e.g., the set of dyadic rational numbers. The system $\tilde{\mathscr{T}}_{D}$ is a countable system of bounded functions contained in $L^{1}(\nu) \cap L^{2}(\nu)$. Moreover, $\tilde{\mathscr{T}}_{D}$ is total in $L^{2}(\nu)$. To see it is enough to observe that the system

$$
\mathscr{T}:=\left\{1_{(a, b]}, \quad 0 \notin[a, b], \quad a, b \in \mathbb{R}\right\}
$$

is total in $L^{2}(\nu)$ as a consequence of Lemma 1.1.8. Because $D$ is dense in $\mathbb{R}$, it follows that $\mathscr{T}$ is contained in the closure in $L^{2}(\nu)$ of $\operatorname{Span}\left(\tilde{\mathscr{T}}_{D}\right)$ which yields $\tilde{\mathscr{T}}_{D}$ is total is $L^{2}(\nu)$. From Theorem 4.2.5 the family

$$
\mathscr{X}_{\tilde{\mathscr{T}}_{D}}:=\left\{\mathrm{W}^{\sigma}\right\} \cup\left\{X^{(f)}, \quad f \in \tilde{\mathscr{T}}_{D}\right\}
$$

possesses the $\mathscr{H}^{2}$-PRP with respect to $\mathbb{F}^{L}$. We observe that any martingale $X^{(f)}$, with $f \in \tilde{\mathscr{T}}_{D}$, is a compensated homogeneous Poisson process. Of course $\mathscr{X}_{\tilde{\mathscr{T}}_{D}}$ is, in general, not a minimal family. On the other side, because $\tilde{\mathscr{T}}_{D}$ is countable, we can orthogonalize it by the Gram-Schmidt orthogonalization procedure. Let $\mathscr{T}_{D}:=\left\{f_{n}, n \geq 1\right\}$ be such an orthogonalization. We introduce the family of locally square integrable martingales $\mathscr{X}_{\mathscr{T}_{D}}:=\left\{\mathrm{W}^{\sigma}\right\} \cup\left\{X^{\left(f_{n}\right)}, n \geq 1\right\}$ which is a minimal family possessing the $\mathscr{H}^{2}$-PRP with respect to $\mathbb{F}^{L}$ (cf. Proposition 4.2.12). From Theorem 4.2.8, any martingale $M \in \mathscr{H}_{0}^{2}\left(\mathbb{F}^{L}\right)$ can be represented as

$$
M=\phi \cdot \mathrm{W}^{\sigma}+\sum_{n=1}^{\infty} H^{n} \cdot X^{\left(f_{n}\right)}, \quad \phi \in \mathrm{L}^{2}\left(\mathrm{~W}^{\sigma}\right), \quad H^{n} \in \mathrm{L}^{2}\left(X^{\left(f_{n}\right)}\right), \quad n \geq 1
$$

and, from Theorem 4.2.10, the integrands in (5.1) are $\lambda \otimes \mathbb{P}$-a.e. uniquely determined by $M$. Each function $f_{n} \in \mathscr{T}_{D}$ is linear combination of functions in $\tilde{\mathscr{T}}_{D}$ and therefore the martingale $X^{\left(f_{n}\right)}$ is linear combination of compensated homogeneous Poisson processes, for every $n \geq 1$. We notice that $\mathscr{X}_{\mathscr{T}_{D}}$ is a family of locally bounded martingales, because $\mathscr{T}_{D} \subseteq L^{2}(\nu)$ is a system of bounded functions (cf. Theorem 2.2.16).

\subsection{Teugels Martingales}

This example shows that under some rather strong assumptions on the Lévy measure $\nu$, Teugels martingales can be introduced as a family possessing the $\mathscr{H}^{2}$-PRP with respect to $\mathbb{F}^{L}$. The $\mathscr{H}^{2}$-PRP for Teugels martingales was established in Nualart \& Schoutens (2000) and we are going to deduce it as an example of the theory which we developed.

Let $\left(L, \mathbb{F}^{L}\right)$ be a Lévy process with characteristic triplet $\left(\beta, \sigma^{2}, \nu\right)$. Teugels martingales are related with polynomials. Indeed, they can be defined if and only if $x^{n} \in L^{2}(\nu)$ for every $n \geq 1$. Furthermore, the family of Teugels martingales possesses the $\mathscr{H}^{2}$-PRP with respect to $\mathbb{F}^{L}$ if and only if the family $\left\{x^{n}, n \geq 1\right\}$ is total in $L^{2}(\nu)$. The following lemma can be obtained as an immediate consequence of Theorem B.4.

5.2.1 Lemma. Let $\mu$ be a finite measure on $(\mathbb{R}, \mathscr{B}(\mathbb{R}))$. If there exists $\xi>0$ such that $\mathrm{e}^{\xi|x|} \in L^{2}(\mu)$, then the family $\mathscr{T}_{1}:=\left\{1, x, x^{2}, \ldots\right\}$ is total in $L^{2}(\mu)$. 
We require the following condition for the Lévy measure $\nu$.

5.2.2 Assumption. There exist two constants $\varepsilon, \lambda>0$ such that

$$
\mathrm{e}^{\frac{\lambda}{2}|x|} 1_{\{|x|>\varepsilon\}} \in L^{2}(\nu) .
$$

The next proposition explains the first consequences of Assumption 5.2.2.

5.2.3 Proposition. Let $\nu$ be a Lévy measure on $(\mathbb{R}, \mathscr{B}(\mathbb{R}))$ satisfying Assumption 5.2.2. Then

(i) $x^{n} \in L^{1}(\nu) \cap L^{2}(\nu)$ for every $n \geq 2$.

(ii) $x \in L^{2}(\nu)$.

(iii) For every fixed $m \geq 1$, the system $\left\{x^{n}, n \geq m\right\}$ is total in $L^{2}(\nu)$.

Proof. To begin with, we verify the first two claims. For a fixed $n \geq 1$ we have

$$
\nu\left(x^{2 n}\right)=\nu\left(1_{\{|x| \leq \varepsilon\}} x^{2 n}\right)+\nu\left(1_{\{|x|>\varepsilon\}} x^{2 n}\right) .
$$

The first term on the right-hand side of the previous formula can be controlled by $c_{\varepsilon, n} \nu\left(1_{\{|x| \leq 1\}} x^{2}\right)$ and the second one by $C_{\varepsilon, n} \nu\left(1_{\{|x|>1\}} \mathrm{e}^{\frac{\lambda}{2}|x|}\right)$, where $c_{\varepsilon, n}$ and $C_{\varepsilon, n}$ are positive constants depending on $\varepsilon$ and $n$. Therefore $\nu\left(x^{2 n}\right)<+\infty$ for every $n \geq 1$. For $n \geq 2$, a similar reasoning yields $x^{n} \in L^{1}(\nu)$. We now show that for every fixed $m \geq 1$ the system $\left\{x^{n}, n \geq m\right\}$ is total in $L^{2}(\nu)$. We define the measure $\nu^{m}$ by

$$
\mathrm{d} \nu^{m}:=\left(x^{m}\right)^{2} \mathrm{~d} \nu, \quad m \geq 1 .
$$

We apply Lemma 1.1.7 with $g(x)=x^{m}$. The function $x^{m}$ belongs to $L^{2}(\nu)$ and so $\nu^{m}$ is a finite measure. In Lemma 1.1.7 the assumption is $g(x) \neq 0$ for every $x$ but now $x^{m}$ is equal to zero in zero. However, this does not cause problems: The point 0 does not play any role because $\nu^{m}(\{0\})=\nu(\{0\})=0$. Thanks to Lemma 1.1.7, to show that $\left\{x^{n}, n \geq m\right\}$ is total in $L^{2}(\nu)$ it is enough to verify that the system $\frac{1}{x^{m}}\left\{x^{n}, n \geq m\right\}=\left\{x^{n}, n \geq 0\right\}$ is total in $L^{2}\left(\nu^{m}\right)$. From Lemma 5.2.1, it is enough to show that there exists $\xi>0$ such that $\mathrm{e}^{\xi|x|} \in L^{2}\left(\nu^{m}\right)$. We have

$$
\nu^{m}\left(\mathrm{e}^{\frac{\lambda}{2}|x|}\right)=\nu\left(x^{2 m} \mathrm{e}^{\frac{\lambda}{2}|x|}\right)=\nu\left(x^{2 m} \mathrm{e}^{\frac{\lambda}{2}|x|} 1_{\{|x| \leq \varepsilon\}}\right)+\nu\left(x^{2 m} \mathrm{e}^{\frac{\lambda}{2}|x|} 1_{\{|x|>\varepsilon\}}\right) .
$$

The function $x^{2 m} \mathrm{e}^{\lambda|x|} 1_{\{|x| \leq \varepsilon\}}$ can be controlled by $c_{\varepsilon} x^{2} 1_{\{|x| \leq 1\}}, c_{\varepsilon}>0$, and so the first summand in the last equality is finite. By Assumption 5.2.2, the function $\mathrm{e}^{\frac{\lambda}{2}|x|} 1_{\{|x|>\varepsilon\}}$ belongs to $L^{2}(\nu)$ and, by Proposition $5.2 .3, x^{n} \in L^{2}(\nu)$ for every $n \geq 1$. Therefore, by Cauchy-Schwarz inequality, the function $x^{2 m} \mathrm{e}^{\frac{\lambda}{2}|x|} 1_{\{|x|>\varepsilon\}}$ is integrable with respect to $\nu$. Hence $\nu^{m}\left(\mathrm{e}^{\frac{\lambda}{2}|x|}\right)<+\infty$, meaning that $\mathrm{e}^{\frac{\lambda}{4}|x|} \in L^{2}\left(\nu^{m}\right)$. We can now apply Lemma 5.2.1 and conclude that $\left\{x^{n}, n \geq 0\right\}$ is total in $L^{2}\left(\nu^{m}\right)$.

Because of Proposition 5.2.3, we can introduce the martingales $X^{(n)}=\left(X_{t}^{(n)}\right)_{t \geq 0}$ as

$$
X_{t}^{(n)}:=\overline{\mathrm{M}}\left(1_{[0, t]} x^{n}\right) \quad t \geq 0, \quad n \geq 1 .
$$

We write $X^{(n)}$ to keep notations simpler. Indeed, to be consistent with the notations introduced in the previous chapters, we should write $X^{\left(p_{n}\right)}$, where the function $p_{n}$ 
is defined by $p_{n}(x):=x^{n}, n \geq 1$. Note that $X^{(1)}$ is an exceptional case because, in general, $x \notin L^{1}(\nu)$. For $n \geq 2, x^{n} \in L^{1}(\nu) \cap L^{2}(\nu)$ so that $X^{(n)}$ is of finite variation and in view of Proposition 2.2.12

$$
X_{t}^{(n)}=\mathrm{M}\left(1_{[0, t]} x^{n}\right)-t \nu\left(x^{n}\right), \quad t \geq 0, \quad n \geq 2 .
$$

With the subsystem of $L^{2}(\nu)$

$$
\mathscr{T}:=\left\{x^{n}, \quad n \geq 2\right\}
$$

we associate the family of martingales

$$
\overline{\mathscr{X}}_{\mathscr{T}}:=\{\bar{L}\} \cup\left\{X^{(n)}, \quad n \geq 2\right\},
$$

where $\bar{L}$ has been defined in (4.38): $\overline{\mathscr{X}}_{\mathscr{T}}$ is the family of Teugels martingales. By Theorem 4.2.14, $\overline{\mathscr{X}}_{\mathscr{T}}$ has the $\mathscr{H}^{2}$-PRP with respect to $\mathbb{F}^{L}$, i.e.,

$$
\mathscr{L}^{2}\left(\overline{\mathscr{X}}_{\mathscr{T}}\right)=\mathscr{H}_{0}^{2}\left(\mathbb{F}^{L}\right) \text {. }
$$

From Proposition 5.2.3, it is clear that the family $\overline{\mathscr{X}}_{\mathscr{T}}$ is not minimal. Indeed, if we consider the system $\mathscr{T}^{m}:=\left\{x^{n}, n \geq m\right\}$, where $m>2$ is fixed, we know that $\mathscr{T}^{m}$ is again a total system in $L^{2}(\nu)$ and so the associated family $\overline{\mathscr{X}}_{\mathscr{T}^{m}}$ is another family with the $\mathscr{H}^{2}$-PRP on $\mathbb{F}^{L}$. Obviously, $\overline{\mathscr{X}}_{\mathscr{T} m} \subsetneq \overline{\mathscr{X}}_{\mathscr{T}}$, for every $m>2$. The non-minimality of $\overline{\mathscr{X}}_{\mathscr{T}}$ is due to the fact that $\overline{\mathscr{X}}_{\mathscr{T}}$ is not an orthogonal family of martingales. By the procedure presented in Kunita \& Watanabe (1967), we can orthogonalize $\overline{\mathscr{X}}_{\mathscr{T}}$ to achieve an orthogonal family $\overline{\mathscr{X}}_{\mathscr{T}}^{\text {orth }}$ of martingales, the orthogonalized Teugels martingales, possessing the $\mathscr{H}^{2}$-PRP with respect to $\mathbb{F}^{L}$. According to Proposition $4.2 .12, \overline{\mathscr{X}}_{\mathscr{T}}^{\text {orth }}$ is a minimal family and every martingale $M \in \mathscr{H}_{0}^{2}\left(\mathbb{F}^{L}\right)$ can be represented as

$$
M=\phi \cdot \bar{L}+\sum_{n=2}^{\infty} H^{n} \cdot X^{(n)}, \quad \phi \in \mathrm{L}^{2}(\bar{L}), \quad H^{n} \in \mathrm{L}^{2}\left(X^{(n)}\right), \quad n \geq 2 .
$$

If we orthogonalize $\overline{\mathscr{X}}_{\mathscr{T} m}$ we obtain a smaller minimal family $\overline{\mathscr{X}}_{\mathscr{T} m}^{\text {orth }}$ with the same properties of $\overline{\mathscr{X}}_{\mathscr{T}}^{\text {orth }}$.

In Nualart \& Schoutens (2000), the definition of Teugels martingales was given in a slightly different way and now we recall it shortly. It will be clear that the two definitions are indeed equivalent. First of all, we introduce the power-jump processes $Z^{(n)}:=\left(Z_{t}^{(n)}\right)_{t \geq 0}$ by

$$
Z_{t}^{(n)}:= \begin{cases}\sum_{0<s \leq t}\left(\Delta L_{s}\right)^{n}, & n \geq 2 \\ L_{t}, & n=1\end{cases}
$$

We point out that in the special case $\sigma^{2}=0$ and $|x| \wedge 1 \in L^{1}(\nu)$ the Lévy process $L$ is of finite variation and the identity

$$
Z_{t}^{(1)}=\sum_{0<s \leq t} \Delta L_{s}, \quad t \geq 0
$$


holds. For $n \geq 2, x^{n} \in L^{1}(\nu)$ and then the process $\mathrm{M}\left(1_{[0, \cdot]} x^{n}\right)$ is of finite variation so that

$$
\mathrm{M}\left(1_{[0, t]} x^{n}\right)=\sum_{0<s \leq t}\left(\Delta L_{s}\right)^{n} 1_{\Delta L_{s} \neq 0}=\sum_{0<s \leq t}\left(\Delta L_{s}\right)^{n}=Z_{t}^{(n)}, \quad t \geq 0, \quad n \geq 2 .
$$

Hence the processes $\left(Z^{(n)}, \mathbb{F}^{L}\right), n \geq 1$, are Lévy processes relative to $\mathbb{F}^{L}$ (cf. Proposition 2.2.15) and, in view of Lemma 2.2.9 and of Theorem 2.3.8,

$$
\mathbb{E}\left[Z_{t}^{(n)}\right]:= \begin{cases}t \nu\left(x^{n}\right), & n \geq 2, \\ t \nu\left(1_{\{|x|>1\}} x\right), & n=1 .\end{cases}
$$

We can then compensate the power-jump processes $Z^{(n)}$ to martingales (cf. Lemma 2.1.1) by

$$
Y_{t}^{(n)}:=Z_{t}^{(n)}-\mathbb{E}\left[Z_{t}^{(n)}\right], \quad t \geq 0, \quad n \geq 1,
$$

and the processes $Y^{(n)}=\left(Y_{t}^{(n)}\right)_{t \geq 0}$ are the Teugels martingales as they were introduced in Nualart \& Schoutens (2000).

\subsection{Lévy Measures Equivalent to the Lebesgue Measure}

We consider a Lévy process $\left(L, \mathbb{F}^{L}\right)$ with characteristic triplet $\left(\beta, \sigma^{2}, \nu\right)$ where $\nu$ is of the form

$$
\mathrm{d} \nu=h \mathrm{~d} x, \quad h>0 .
$$

An important class of Lévy processes with Lévy measure as in (5.7) is, for example, the class of $\alpha$-stable processes (see, e.g., Sato (1999), Chapter 3). We begin with the simpler case of the Cauchy process. A Cauchy process $\left(L, \mathbb{F}^{L}\right)$ is a purely nonGaussian Lévy process with characteristic triplet $(0,0, \nu)$ and

$$
\mathrm{d} \nu:=\frac{1}{x^{2}} \mathrm{~d} x
$$

where, for simplicity, we did not consider multiplicative constants. For a Cauchy process no moment is defined and therefore, the Lévy measure does not satisfy Assumption 5.2.2. Hence the problem of finding a family of martingales with the $\mathscr{H}^{2}$-PRP cannot be solved by means of Teugels martingales. Nevertheless, we are able to introduce families of orthogonal locally bounded martingales possessing the $\mathscr{H}^{2}$-PRP with respect to $\mathbb{F}^{L}$. According to Theorem 4.2.5, for this goal it is enough to find a complete orthogonal system in $L^{2}(\nu)$ consisting of bounded functions. Thanks to Lemma 1.1.7, for this it is sufficient to consider the problem of finding a total system in $L^{2}\left(\nu^{g}\right)$, where $\nu^{g}$ is a suitable finite measure equivalent to $\nu$. We choose the function $g$ as $g(x):=x \exp \left(-\frac{x^{2}}{2}\right), x \in \mathbb{R}$. Obviously, $g \in L^{2}(\nu)$ and so the measure $\nu^{g}$ defined by

$$
\mathrm{d} \nu^{g}:=g^{2} \mathrm{~d} \nu=\mathrm{e}^{-x^{2}} \mathrm{~d} x
$$


is a finite measure on $(\mathbb{R}, \mathscr{B}(\mathbb{R}))$. Let $\left(H_{n}\right)_{n=0,1, \ldots}$ be the sequence of Hermite polynomials and define

$$
P_{n}:=\left(\pi^{\frac{1}{4}}\left(2^{n} n !\right)^{\frac{1}{2}}\right) H_{n}, \quad n \geq 0 .
$$

The sequence $\left(P_{n}\right)_{n \geq 0}$ is an orthonormal basis for $L^{2}\left(\nu^{g}\right)$. Indeed, the measure $\nu^{g}$ is a weighted Lebesgue measure and the Hermite polynomial $H_{n}$ is the eigenfunction of a Sturm-Liouville equation on $(-\infty,+\infty)$ relative to the eigenvalue $\lambda_{n}=n$. Therefore, because of Lemma 1.1.7, the system $\mathscr{T}=\left\{C_{n}, n \geq 0\right\} \subseteq L^{2}(\nu)$, where

$$
C_{n}:=g P_{n}, \quad n \geq 0
$$

is a complete orthonormal system in $L^{2}(\nu)$. Moreover, each $C_{n}$ is a bounded function. In view of Theorem 2.2.16 and Theorem 4.2.5, the family $\mathscr{X}_{\mathscr{T}}:=\left\{X^{\left(C_{n}\right)}, n \geq 0\right\}$ is a family of locally bounded orthogonal martingales with the $\mathscr{H}^{2}$-PRP with respect to $\mathbb{F}^{L}$. According to Proposition $4.2 .12, \mathscr{X}_{\mathscr{T}}$ is a minimal family in $\mathscr{H}_{0}^{2}$. Moreover, every $M \in \mathscr{H}_{0}^{2}\left(\mathbb{F}^{L}\right)$ can be represented as

$$
M=\sum_{n=0}^{\infty} H^{n} \cdot X^{\left(C_{n}\right)}, \quad H^{n} \in \mathrm{L}^{2}\left(X^{\left(C_{n}\right)}\right), \quad n \geq 0,
$$

and the integrands are $\lambda_{+} \otimes \mathbb{P}$-a.e. uniquely determined by $M$ (cf. Theorem 4.2.8 and Theorem 4.2.10).

\subsubsection{Hermite Polynomials}

We have seen that for the Cauchy process Hermit polynomials can be used to generate a family of orthogonal martingales with the $\mathscr{H}^{2}$-PRP. Now we consider the general case of a Lévy process with Lévy measure of the form (5.7). Let $\left(L, \mathbb{F}^{L}\right)$ be a Lévy process with characteristics $\left(\beta, \sigma^{2}, \nu\right)$ where $\nu$ is as in (5.7). We choose the function $g$ as $g(x):=\frac{1}{\sqrt{h}} \exp \left(-\frac{x^{2}}{2}\right)$, which belongs to $L^{2}(\nu)$, and define the finite measure

$$
\mathrm{d} \nu^{g}:=g^{2} \mathrm{~d} \nu
$$

The polynomials $\left(P_{n}\right)_{n=0,1, \ldots}$ introduced as in (5.8) are a complete orthonormal system in $L^{2}\left(\nu^{g}\right)$ and the system $\mathscr{T}=\left\{C_{n}, n \geq 0\right\}$, where

$$
C_{n}:=g P_{n}, \quad n \geq 0,
$$

is a complete orthogonal system in $L^{2}(\nu)$. For a general $h>0$, we cannot expect that this is a system of bounded functions. Then $\mathscr{X}_{\mathscr{T}}:=\left\{\mathrm{W}^{\sigma}\right\} \cup\left\{X^{\left(C_{n}\right)}, n \geq 0\right\}$ is an orthogonal family of martingales and it has the $\mathscr{H}^{2}$-PRP with respect to $\mathbb{F}^{L}$. Because of the orthogonality, this is also a minimal family in $\mathscr{H}_{0}^{2}$. Moreover, every $M \in \mathscr{H}_{0}^{2}\left(\mathbb{F}^{L}\right)$ can be represented as

$$
M=\phi \cdot \mathrm{W}^{\sigma}+\sum_{n=0}^{\infty} H^{n} \cdot X^{\left(C_{n}\right)}, \quad \phi \in \mathrm{L}^{2}\left(\mathrm{~W}^{\sigma}\right), \quad H^{n} \in \mathrm{L}^{2}\left(X^{\left(C_{n}\right)}\right), \quad n \geq 0,
$$

and the integrands are $\lambda_{+} \otimes \mathbb{P}$-a.e. uniquely determined by $M$ (cf. Theorem 4.2.8 and Theorem 4.2.10). 


\subsubsection{Haar Wavelet}

Haar functions are well known in functional analysis and in particular in the theory of function spaces. For this part we refer to Wojtaszczyk (1997), Chapter 1. We consider a Lévy process $\left(L, \mathbb{F}^{L}\right)$ with characteristics $\left(\beta, \sigma^{2}, \nu\right)$ and we assume that the Lévy measure $\nu$ is as in (5.7). Let $\lambda$ be the Lebesgue measure on $(\mathbb{R}, \mathscr{B}(\mathbb{R}))$ and $\psi \in L^{2}(\lambda)$. If the system $\left\{\psi_{j k}:=2^{\frac{j}{2}} \psi\left(2^{j} x-k\right), x \in \mathbb{R}, j, k \in \mathbb{Z}\right\}$ is a complete orthonormal system of $L^{2}(\lambda)$, the function $\psi$ is called a wavelet. An example of a wavelet is the function

$$
\psi(x):=\left\{\begin{aligned}
1, & x \in\left[0, \frac{1}{2}\right) \\
-1, & x \in\left[\frac{1}{2}, 1\right) \\
0, & \text { otherwise }
\end{aligned}\right.
$$

known as Haar wavelet while the system $\tilde{\mathscr{T}}:=\left\{\psi_{j k}, j, k \in \mathbb{Z}\right\}$ generated by it is the Haar basis ${ }^{1}$ (cf. Wojtaszczyk (1997)). The generic element of the Haar basis can be written as

$$
\psi_{j k}(x):=2^{\frac{j}{2}}\left[1_{\left[\frac{k}{2^{j}}, \frac{2 k+1}{2^{j+1}}\right)}(x)-1_{\left[\frac{2 k+1}{2^{j+1}}, \frac{k+1}{2^{j}}\right)}(x)\right]
$$

and its support is $\left\{\psi_{j k} \neq 0\right\}=\left[\frac{k}{2^{j}}, \frac{k+1}{2^{j}}\right], j, k \in \mathbb{Z}$. Note that the boundary points of these intervals are dyadic rational numbers. By Lemma 1.1.7, $\mathscr{T}:=\frac{1}{\sqrt{h}} \tilde{\mathscr{T}}$ is a complete orthogonal system in $L^{2}(\nu)$. For a general $h>0$ we cannot expect that this is a system of bounded functions. The family $\mathscr{X}_{\mathscr{T}}:=\left\{\mathrm{W}^{\sigma}\right\} \cup\left\{X^{(f)}, \quad f \in \mathscr{T}\right\}$ is a family of orthogonal martingales possessing the $\mathscr{H}^{2}$-PRP with respect to $\mathbb{F}^{L}$. Because of the orthogonality this is also a minimal family in $\mathscr{H}_{0}^{2}$. Moreover, every $M \in \mathscr{H}_{0}^{2}\left(\mathbb{F}^{L}\right)$ can be represented as

$$
M=\phi \cdot \mathrm{W}^{\sigma}+\sum_{n=0}^{\infty} H^{n} \cdot X^{\left(f_{n}\right)}, \quad \phi \in \mathrm{L}^{2}\left(\mathrm{~W}^{\sigma}\right), \quad H^{n} \in \mathrm{L}^{2}\left(X^{\left(f_{n}\right)}\right), \quad n \geq 0
$$

and the integrands are $\lambda_{+} \otimes \mathbb{P}$-a.e. uniquely determined by $M$ (cf. Theorem 4.2.8 and Theorem 4.2.10). It is clear that the previous reasoning can be repeated for every wavelet $\psi \in L^{2}(\lambda)$.

5.3.1 Remark. It is not difficult to show that, also in the case of a general Lévy measure $\nu$, the Haar system appropriately transformed leads to a complete orthogonal system in $L^{2}(\nu)$. For reasons of shortness, we did not consider in this work the general case and we discussed only the situation in which the Lévy measure is equivalent to the Lebesgue measure as in (5.7).

\footnotetext{
${ }^{1}$ The idea of using the Haar basis as a total system in $L^{2}(\lambda)$ was suggested by Lev Markhasin.
} 

APPENDIX A

Complement to Additive Processes

In this appendix we fix a complete probability space $(\Omega, \mathscr{F}, \mathbb{P})$ and a filtration $\mathbb{F}$ of events of $\Omega$ which is assumed to satisfy the usual condition. In $\S$ A.1 we verify Theorem 2.1.8 and its converse, while in $\S$ A.2 we show the Itô-Lévy decomposition.

\section{A.1. Independence of Additive Processes}

In He, Wang \& Yan (1992), Theorem 11.43, the following result is established.

A.1.1 Theorem. Let $X^{1}, \ldots, X^{m}$ be additive processes and semimartingales. If

$$
\left[X^{j}, X^{k}\right]=0, \quad j, k=1, \ldots, m ; \quad j \neq k,
$$

then the vector $\left(X^{1}, \ldots, X^{m}\right)$ is independent.

In He, Wang \& Yan (1992), in the remark to Theorem 11.43, it is asserted that also the converse of Theorem A.1.1 holds, but the proof is left as an exercise. In this part we give a detailed proof of Theorem A.1.1 for additive process and of its converse in the special case of Lévy processes. We shall proceed in a different way from He, Wang \& Yan (1992). We consider processes which are semimartingales and additive processes relative to the filtration $\mathbb{F}$ and we prove the following result:

A.1.2 Theorem. Let $X^{1}, \ldots, X^{m}$ be additive processes relative to $\mathbb{F}$ and semimartingales. If

$$
\left[X^{j}, X^{k}\right]=0, \quad j, k=1, \ldots, m ; j \neq k,
$$

then the vector $\left(X^{1}, \ldots, X^{m}\right)$ has $\mathbb{F}$-independent increments, that is, the random vector $\left(X_{t}^{1}-X_{s}^{1}, \ldots, X_{t}^{m}-X_{s}^{m}\right)$ is independent of $\mathscr{F}_{s}$, for every $0 \leq s \leq t$.

Theorem A.1.2 is stronger than Theorem A.1.1 and we shall obtain the latter result as a corollary of the first one. Before to give the proof of Theorem A.1.2, we 
need some preparation. Let $x_{1}, \ldots, x_{m}$ and $x_{1-}, \ldots, x_{m-}$ be two sequence of real numbers. We define

$$
\Delta x_{j}:=x_{j}-x_{j-}, \quad j=1, \ldots, m ; \quad \Delta \prod_{j=1}^{m} x_{j}:=\prod_{j=1}^{m} x_{j}-\prod_{j=1}^{m} x_{j-} .
$$

We now state a purely algebraic result.

A.1.3 Lemma. For any two sequences $x_{1}, \ldots, x_{m}$ and $x_{1-}, \ldots, x_{m-}$ of real numbers such that $\Delta x_{j} \Delta x_{k}=0, j, k=1, \ldots, m, j \neq k$, it follows:

$$
\Delta \prod_{j=1}^{m} x_{j}=\sum_{j=1}^{m}\left(\prod_{k \neq j}^{m} x_{k-}\right) \Delta x_{j} .
$$

Proof. We proceed by induction on $m \in \mathbb{N}$. If $m=1$ there is nothing to show. Now we assume (A.1) for $m=n$ and show it for $m=n+1$.

$$
\begin{aligned}
\Delta & \prod_{j=1}^{n+1} x_{j}=\prod_{j=1}^{n+1} x_{j}-\prod_{j=1}^{n+1} x_{j-} \\
& =x_{n+1} \prod_{j=1}^{n} x_{j}-x_{(n+1)-} \prod_{j=1}^{n} x_{j}+x_{(n+1)-} \prod_{j=1}^{n} x_{j}-x_{(n+1)-} \prod_{j=1}^{n} x_{j-} \\
& =\Delta x_{n+1}\left(\prod_{j=1}^{n} x_{j-}+\prod_{j=1}^{n} x_{j}-\prod_{j=1}^{n} x_{j-}\right)+x_{(n+1)-}\left(\prod_{j=1}^{n} x_{j}-\prod_{j=1}^{n} x_{j-}\right) \\
& =\Delta x_{n+1}\left(\prod_{j=1}^{n} x_{j-}+\sum_{j=1}^{n}\left(\prod_{k \neq j} x_{j-}\right) \Delta x_{j}\right)+x_{(n+1)-} \sum_{j=1}^{n}\left(\prod_{k \neq j} x_{k-}\right) \Delta x_{j} \\
& =\sum_{j=1}^{n+1}\left(\prod_{k \neq j} x_{k-}\right) \Delta x_{j},
\end{aligned}
$$

where in the last but one equality we used the induction hypothesis.

Let $\left(X^{j}, \mathbb{F}\right), j=1, \ldots, m$, be additive processes. With $\left(X^{j}, \mathbb{F}\right)$ we associate the $\mathbb{F}$-martingale $Z^{j}:=\left(Z_{t}^{j}\right)_{t \geq 0}$ defined by

$$
Z_{t}^{j}:=\varphi_{t}^{j}\left(u^{j}\right)^{-1} \exp \left(i u^{j} X_{t}^{j}\right), \quad t \geq 0, \quad u^{j} \in \mathbb{R}, \quad j=1, \ldots, m .
$$

We call the $\mathbb{F}$-martingale $Z^{j}$, the martingale associated with the process $X^{j}, j=$ $1, \ldots, m$. We remark that the process $Z^{j}$ depends also on the parameter $u^{j}$ and so we should write $Z^{j}\left(u^{j}\right)$ but we write only $Z^{j}$ to keep notations simpler.

A.1.4 Lemma. Let $X^{1}, \ldots, X^{m}$ be additive processes relative to $\mathbb{F}$ and semimartingales. For the martingales $Z^{1}, \ldots, Z^{m}$ associated with $X^{1}, \ldots, X^{m}$ it follows that $\left[Z^{j}, Z^{k}\right]=0, j, k=1, \ldots, m, k \neq j$, if and only if $\left[X^{j}, X^{k}\right]=0, j, k=1, \ldots, m$, $k \neq j$.

Proof. We preliminary observe that for any semimartingales $X$ and $Y$ we have $[X, Y]=0$ if and only if $\left\langle X^{c}, Y^{c}\right\rangle=0$ and $\Delta X \Delta Y=0$ (cf. $\S 1.2 .8$ ). Let $\varphi_{t}^{j}$ be the characteristic function of $X_{t}^{j}$. We already know that $\varphi_{t}^{j}\left(u^{j}\right)$ is continuous in both $t$ and $u^{j}$. Moreover, because $X^{j}$ is a semimartingale, $\varphi_{t}^{j}\left(u^{j}\right)$ has finite variation, for every $u^{j} \in \mathbb{R}$ (cf. Theorem 2.1.7). Because of $\varphi_{t}^{j}\left(u^{j}\right) \neq 0, j=1, \ldots, m$, for every $u^{j} \in \mathbb{R}$ and $t \geq 0$, we can define $\varphi_{t}^{j}\left(u^{j}\right)^{-1}$ which is continuous and of finite variation. Therefore, for every semimartingale $S$, we have $\left[\varphi^{j}\left(u^{j}\right)^{-1}, S\right]=0$. Now 
we introduce the process $Y^{j}:=\exp \left(i u^{j} X^{j}\right)$. By the formula of integration by parts (cf. (1.35)) and the previous considerations, we have

$$
\begin{aligned}
Z_{t}^{j}=Y_{t}^{j} \varphi_{t}^{j}\left(u^{j}\right)^{-1} & =1+Y_{-}^{j} \cdot \varphi_{t}^{j}\left(u^{j}\right)^{-1}+\varphi^{j}\left(u^{j}\right)^{-1} \cdot Y_{t}^{j}+\left[\varphi^{j}\left(u^{j}\right)^{-1}, Y^{j}\right]_{t} \\
& =1+Y_{-}^{j} \cdot \varphi_{t}^{j}\left(u^{j}\right)^{-1}+\varphi^{j}\left(u^{j}\right)^{-1} \cdot Y_{t}^{j} .
\end{aligned}
$$

By Proposition 1.3.6 we get

$$
\left(Z^{j}\right)^{c}=\left(\varphi^{j}\left(u^{j}\right)^{-1} \cdot Y^{j}\right)^{c}=\varphi^{j}\left(u^{j}\right)^{-1} \cdot\left(Y^{j}\right)^{c} .
$$

Itô's formula applied to $Y^{j}$ yields $\left(Y^{j}\right)^{c}=i u^{j} Y^{j} \cdot\left(X^{j}\right)^{c}$ and inserting this in (A.3) we obtain $\left(Z^{j}\right)^{c}=i u^{j} Z^{j} \cdot\left(X^{j}\right)^{c}$. Consequently

$$
\left\langle\left(Z^{j}\right)^{c},\left(Z^{k}\right)^{c}\right\rangle=-u^{j} u^{k}\left(Z^{j} Z^{k}\right) \cdot\left\langle\left(X^{j}\right)^{c},\left(X^{k}\right)^{c}\right\rangle .
$$

Let us now compute $\Delta Z^{j} \Delta Z^{k}$. By the definition of $Z^{j}$ and $Z^{k}$, for every $t \geq 0$ a.s., we get

$$
\left|\Delta Z_{t}^{j} \Delta Z_{t}^{k}\right|=\left|\exp \left(i u^{j} \Delta X_{t}^{j}\right)-1\right|\left|\exp \left(i u^{k} \Delta X_{t}^{k}\right)-1\right| \times\left|\varphi_{t}^{j}\left(u^{j}\right)^{-1}\right|\left|\varphi_{t}^{k}\left(u^{k}\right)^{-1}\right| .
$$

We now prove that $\left[X^{j}, X^{k}\right]=0, j, k=1, \ldots, m, k \neq j$, yields $\left[Z^{j}, Z^{k}\right]=0$, $j, k=1, \ldots, m, k \neq j$. The fact $\left[X^{j}, X^{k}\right]=0, j \neq k$, implies that $\left\langle\left(X^{j}\right)^{c},\left(X^{k}\right)^{c}\right\rangle=0$, $j \neq k$. But this, together with (A.4), yields $\left\langle\left(Z^{j}\right)^{c},\left(Z^{k}\right)^{c}\right\rangle=0, j \neq k$. Let us now consider (A.5). The fact $\left[X^{j}, X^{k}\right]=0$ implies $\Delta X^{j} \Delta X^{k}=0, j \neq k$. The last factor on the right-hand side of (A.5) cannot vanish and the first is equal to zero because $\Delta X^{j} \Delta X^{k}=0$. Therefore $\Delta Z^{j} \Delta Z^{k}=0, j \neq k$. Hence from the definition of $\left[Z^{j}, Z^{k}\right]$, we obtain that $\left[Z^{j}, Z^{k}\right]=0, j, k=1, \ldots, m, k \neq j$. Conversely, we now assume that $\left[Z^{j}, Z^{k}\right]=0 j, k=1, \ldots, m, k \neq j$, and show that $\left[X^{j}, X^{k}\right]=0$, $j, k=1, \ldots, m, k \neq j$. The martingale $Z^{j}$ is always different from zero. Therefore from (A.4), we get

$$
\left\langle\left(X^{j}\right)^{c},\left(X^{k}\right)^{c}\right\rangle=-u^{j} u^{k}\left(Z^{j} Z^{k}\right)^{-1} \cdot\left\langle\left(Z^{j}\right)^{c},\left(Z^{k}\right)^{c}\right\rangle
$$

and $\left\langle\left(Z^{j}\right)^{c},\left(Z^{k}\right)^{c}\right\rangle=0$ issues $\left\langle\left(X^{j}\right)^{c},\left(X^{k}\right)^{c}\right\rangle=0, j, k=1, \ldots, m, k \neq j$. The left-hand side of (A.5) is equal to zero and the second factor on the right-hand side cannot vanish. Therefore the first factor on the right-hand side of (A.5) must vanish and this is possible if and only if $\Delta X^{j} \Delta X^{k}=0, j, k=1, \ldots, m, k \neq j$. In conclusion, we showed that $\left[Z^{j}, Z^{k}\right]=0, j, k=1, \ldots, m, k \neq j$, implies $\left[X^{j}, X^{k}\right]=0$, $j, k=1, \ldots, m, k \neq j$ and the proof is finished.

Let $X^{1}, \ldots, X^{m}$ be additive processes relative to $\mathbb{F}$ and $Z^{1}, \ldots, Z^{m}$ be the associated martingales (cf. (A.2)). We define the process $Z=\left(Z_{t}\right)_{t \geq 0}$ by

$$
Z:=\prod_{j=1}^{m} Z^{j} .
$$

We now state a lemma which gives sufficient conditions for the process $Z$ to be a martingale. 
A.1.5 Lemma. Let $X^{1}, \ldots, X^{m}$ be semimartingales and additive processes relative to $\mathbb{F}$. If $\left[X^{j}, X^{k}\right]=0, j, k=1, \ldots, m, k \neq j$, then $Z$ defined in (A.6) is an $\mathbb{F}$ martingale with $Z_{0}=1$.

Proof. We consider the function $F\left(x_{1}, \ldots, x_{m}\right):=\prod_{j=1}^{m} x^{j}$. Then

$$
Z=F\left(Z^{1}, \ldots, Z^{m}\right)
$$

Applying Itô's formula, we get

$$
\begin{aligned}
Z_{t}=1 & +\sum_{r=1}^{m} \frac{\partial}{\partial x_{r}} F\left(Z_{-}^{1}, \ldots, Z_{-}^{m}\right) \cdot Z_{t}^{r} \\
& +\sum_{\substack{r, q=1, q \neq r}}^{m} \frac{\partial^{2}}{\partial x_{r} \partial x_{q}} F\left(Z^{1}, \ldots, Z^{m}\right) \cdot\left\langle\left(Z^{r}\right)^{\mathrm{c}},\left(Z^{q}\right)^{\mathrm{c}}\right\rangle_{t} \\
& +\sum_{0<s \leq t}\left\{\Delta F\left(Z_{s}^{1}, \ldots, Z_{s}^{m}\right)-\sum_{r=1}^{m} \frac{\partial}{\partial x_{r}} F\left(Z_{s-}^{1}, \ldots, Z_{s-}^{m}\right) \Delta Z_{s}^{r}\right\} .
\end{aligned}
$$

Because of Lemma A.1.4, the process $\left[Z^{j}, Z^{k}\right]$ vanishes, for every $j \neq k$. This implies that $\Delta Z^{j} \Delta Z^{k}$ and $\left\langle\left(Z^{j}\right)^{c},\left(Z^{k}\right)^{c}\right\rangle$ are evanescent, for all $j \neq k$. Therefore the second sum on the right-hand side of the previous formula vanishes. Moreover, thanks to Lemma A.1.3 we can conclude that also the last sum vanishes. Hence

$$
Z_{t}=1+\sum_{r=1}^{m} \frac{\partial}{\partial x_{r}} F\left(Z^{1}, \ldots, Z^{m}\right)_{-} \cdot Z_{t}^{r}
$$

which implies, in particular, that $Z$ is a local martingale. Indeed, $\frac{\partial}{\partial x_{r}} F\left(Z_{-}^{1}, \ldots, Z_{-}^{m}\right)$ is locally bounded and predictable and so it belongs to $\mathrm{L}_{\text {loc }}^{2}\left(Z^{r}\right)$ (cf. (1.26)), for every $r=1, \ldots, m$. On the other side, we have

$$
\left|Z_{t}^{j}\right|=\left|\varphi_{t}^{j}\left(u_{j}\right)^{-1}\right|, \quad t \geq 0
$$

and $t \mapsto \varphi_{t}^{j}\left(u_{j}\right)^{-1}$ is a continuous function, therefore it is bounded on every compact interval. In conclusion $Z$ is bounded on every compact interval and from this it follows that $Z$ must be a true martingale.

Now we are ready to prove Theorem A.1.2.

Proof of Theorem A.1.2. Let $Z^{j}$ be the martingale associated with $X^{j}$ (cf. (A.2)). By Lemma A.1.5 the process $Z:=\prod_{j=1}^{m} Z^{j}$ is an $\mathbb{F}$-martingale and hence, for every $0 \leq s \leq t, \mathbb{E}\left[Z_{t} \mid \mathscr{F}_{s}\right]=Z_{s}$. Because of the definition of $Z^{j}$, this yields, for every $0 \leq s \leq$ and $u^{j} \in \mathbb{R}$,

$$
\frac{\mathbb{E}\left[\exp \left(i \sum_{j=1}^{m} u^{j} X_{t}^{j}\right) \mid \mathscr{F}_{s}\right]}{\prod_{j=1}^{m} \varphi_{t}^{j}\left(u^{j}\right)}=\frac{\exp \left(i \sum_{j=1}^{m} u^{j} X_{s}^{j}\right)}{\prod_{j=1}^{m} \varphi_{s}^{j}\left(u^{j}\right)} .
$$


Hence

$$
\mathbb{E}\left[\exp \left(i \sum_{j=1}^{m} u^{j}\left(X_{t}^{j}-X_{s}^{j}\right)\right) \mid \mathscr{F}_{s}\right]=\prod_{j=1}^{m} \frac{\varphi_{t}^{j}\left(u^{j}\right)}{\varphi_{s}^{j}\left(u^{j}\right)}=\prod_{j=1}^{m} \mathbb{E}\left[\mathrm{e}^{i u^{j}\left(X_{t}^{j}-X_{s}^{j}\right)}\right],
$$

for every $0 \leq s \leq$ and $u^{j} \in \mathbb{R}$. The previous formula implies, in particular, that $\left(X_{t}^{1}-X_{s}^{1}, \ldots, X_{t}^{m}-X_{s}^{m}\right)$ is independent of $\mathscr{F}_{s}$ and the proof is finished.

We now are going to verify that under the assumptions of Theorem A.1.2, the vector $\left(X^{1}, \ldots, X^{m}\right)$ is an independent vector of additive processes. We obtain Theorem A.1.1 as a corollary to Theorem A.1.2. To see this fact we need a preliminary lemma.

A.1.6 Lemma. Let $(X, \mathbb{F})$ be an additive process. For any $0=t_{0}<t_{1}<\ldots<t_{n}$ the process $X^{(n)}=\left(X_{t}^{(n)}\right)_{t \geq 0}$ defined by

$$
X_{t}^{(n)}:=\sum_{j=1}^{n} u_{j}\left(X_{t \wedge t_{j}}-X_{t \wedge t_{j-1}}\right), \quad t \geq 0, \quad u_{j} \in \mathbb{R}, \quad j=1, \ldots, n,
$$

is an additive process relative to $\mathbb{F}$. Moreover, if $X$ is a semimartingale, then $X^{(n)}$ is a semimartingale.

Proof. Clearly, $X^{(n)}$ is adapted, càdlàg and stochastically continuous. It is obvious that if $X$ is a semimartingale, then also $X^{(n)}$ is a semimartingale. Indeed, the process $X^{(n)}$ is linear combination of elements of the form $X^{v}$, where the superscript $v$ denotes the operation of stopping at the deterministic time $v \in \mathbb{R}_{+}$. The relevant property to establish is the $\mathbb{F}$-independence of the increments. To compute the explicit form of the increments of $X^{(n)}$, several cases should be considered. We do not show this tedious computations. However, it is not difficult to see that the increment $X_{t}^{(n)}-X_{s}^{(n)}, 0 \leq s<t$, is a linear transformation of a vector of the form $\left(X_{q_{1}}-X_{s}, \ldots, X_{q_{n}}-X_{s}\right), s<q_{1}<\ldots<q_{n}$. Therefore it is enough to show that, for every fixed $s<q_{1}<\ldots<q_{n}$, such a vector is independent of $\mathscr{F}_{s}$. We consider the vector $\left(X_{q_{1}}-X_{s}, X_{q_{2}}-X_{q_{1}}, \ldots, X_{q_{n}}-X_{q_{n-1}}\right), s<q_{1}<\ldots<q_{n}$, and we show that it is independent of $\mathscr{F}_{s}$ for every $n \in \mathbb{N}$. We proceed by induction. If $n=1$, there is nothing to prove. We assume the statement for $n$ and we show it for $n+1$. For every $B_{1}, \ldots, B_{n+1} \in \mathscr{B}(\mathbb{R})$, by the properties of the conditional expectation, we have

$$
\begin{aligned}
& \mathbb{E}\left[1_{\left\{X_{q_{1}}-X_{s} \in B_{1}, \ldots, X_{q_{n}}-X_{q_{n-1}} \in B_{n}, X_{q_{n+1}}-X_{q_{n}} \in B_{n+1}\right\}} \mid \mathscr{F}_{s}\right] \\
& \quad=\mathbb{E}\left[1_{\left\{X_{q_{1}}-X_{s} \in B_{1}, \ldots, X_{q_{n}}-X_{q_{n-1}} \in B_{n}\right\}} \mathbb{E}\left[1_{\left\{X_{q_{n+1}}-X_{q_{n}} \in B_{n+1}\right\}} \mid \mathscr{F}_{q_{n}}\right] \mid \mathscr{F}_{s}\right] \\
& \quad=\mathbb{E}\left[1_{\left\{X_{q_{1}}-X_{s} \in B_{1}, \ldots, X_{q_{n}}-X_{q_{n-1}} \in B_{n}\right\}} \mathbb{E}\left[1_{\left\{X_{q_{n+1}}-X_{q_{n}} \in B_{n+1}\right\}}\right] \mid \mathscr{F}_{s}\right] \\
& \quad=\mathbb{E}\left[1_{\left\{X_{q_{1}}-X_{s} \in B_{1}\right\}}\right] \prod_{i=2}^{n+1} \mathbb{E}\left[1_{\left\{X_{q_{i}}-X_{q_{i-1}} \in B_{i}\right\}}\right],
\end{aligned}
$$

where, in the second passage, we used that the process $X$ has $\mathbb{F}$-independent increments and in the last passage the induction hypothesis. Then we can conclude that the vector $\left(X_{q_{1}}-X_{s}, X_{q_{2}}-X_{q_{1}}, \ldots, X_{q_{n}}-X_{q_{n-1}}\right), s<q_{1}<\ldots<q_{n}$, is independent of $\mathscr{F}_{s}$ for every $n \in \mathbb{N}$. The vector $\left(X_{q_{1}}-X_{s}, \ldots, X_{q_{n}}-X_{s}\right), s<q_{1}<\ldots<q_{n}$ is a linear transformation of $\left(X_{q_{1}}-X_{s}, X_{q_{2}}-X_{q_{1}}, \ldots, X_{q_{n}}-X_{q_{n-1}}\right)$ and hence it is independent of $\mathscr{F}_{s}$. From this it easily follows that $X^{(n)}$ has $\mathbb{F}$-independent increments, for every $n \geq 1$, and the proof is finished. 
We observe that Lemma A.1.6 does not hold for a Lévy process. Indeed, if $L$ is a Lévy process, then the process $X^{(n)}$ defined in (A.8) is, in general, not a Lévy process because the homogeneity of increments is lost. Nevertheless, $X^{(n)}$ is an additive process. Now we show Theorem A.1.1.

Proof of Theorem A.1.1. Let $\left(t_{j}\right)_{j=0,1, \ldots, n}$ be a sequence of deterministic times such that $0=t_{0}<t_{1}<\ldots<t_{n}$. We define the process $X^{(j, n)}$ by

$$
X_{t}^{(j, n)}:=\sum_{k=1}^{n} u_{k}^{j}\left(X_{t \wedge t_{k}}^{j}-X_{t \wedge t_{k-1}}^{j}\right), \quad t \geq 0
$$

Because of Lemma A.1.6, $X^{(1, n)}, \ldots, X^{(m, n)}$ are additive processes relative to $\mathbb{F}$ and semimartingales. Moreover, they are such that the process $\left[X^{(j, n)}, X^{(k, n)}\right]$ vanishes for every $j, k=1, \ldots, m, j \neq k$. Indeed, $\left[X^{(j, n)}, X^{(k, n)}\right]$ is linear combination of elements of the form $\left[X^{j}, X^{k}\right]^{v}$, where the superscript $v$ denotes the operation of stopping at the deterministic time $v \in \mathbb{R}_{+}$, and, by assumption $\left[X^{j}, X^{k}\right]=0$, $j \neq k$. From Theorem A.1.2, the vector $\left(X_{t}^{(1, n)}-X_{s}^{(1, n)}, \ldots, X_{t}^{(m, n)}-X_{s}^{(m, n)}\right)$ is independent of $\mathscr{F}_{s}$, for every $0 \leq s \leq t$. In particular, writing (A.7) for the vector $\left(X_{t}^{(1, n)}-X_{s}^{(1, n)}, \ldots, X_{t}^{(m, n)}-X_{s}^{(m, n)}\right)$, setting $s=0$ and taking the expectation, we deduce that, for every $t \geq 0$ and $u_{k}^{j} \in \mathbb{R}, j=1, \ldots, m, k=1, \ldots, n$, it follows

$\mathbb{E}\left[\exp \left(i \sum_{j=1}^{m} \sum_{k=1}^{n} u_{k}^{j}\left(X_{t \wedge t_{k}}^{j}-X_{t \wedge t_{k-1}}^{j}\right)\right)\right]=\prod_{j=1}^{m} \mathbb{E}\left[\exp \left(i \sum_{k=1}^{n} u_{k}^{j}\left(X_{t \wedge t_{k}}^{j}-X_{t \wedge t_{k-1}}^{j}\right)\right)\right]$.

For $t>t_{n}$ and for every $u_{k}^{j} \in \mathbb{R}, j=1, \ldots, m, k=1, \ldots, n$, the previous formula becomes

$$
\mathbb{E}\left[\exp \left(i \sum_{j=1}^{m} \sum_{k=1}^{n} u_{k}^{j}\left(X_{t_{k}}^{j}-X_{t_{k-1}}^{j}\right)\right)\right]=\prod_{j=1}^{m} \mathbb{E}\left[\exp \left(i \sum_{k=1}^{n} u_{k}^{j}\left(X_{t_{k}}^{j}-X_{t_{k-1}}^{j}\right)\right)\right],
$$

and this implies that the family $\left\{\left(X_{t_{1}}^{j}, X_{t_{2}}^{j}-X_{t_{1}}^{j}, \ldots, X_{t_{n}}^{j}-X_{t_{n-1}}^{j}\right), j=1, \ldots m\right\}$ is independent. For any fixed $1 \leq j \leq m$ the vector $\left(X_{t_{1}}^{j}, \ldots, X_{t_{n}}^{j}\right)$ is a linear transformation of $\left(X_{t_{1}}^{j}, X_{t_{2}}^{j}-X_{t_{1}}^{j}, \ldots, X_{t_{n}}^{j}-X_{t_{n-1}}^{j}\right)$. Hence we can assert that the family $\left\{\left(X_{t_{1}}^{j}, \ldots, X_{t_{n}}^{j}\right), j=1, \ldots, m\right\}$ is independent for every sequence $\left(t_{j}\right)_{j=0,1, \ldots, n}$, i.e., that the vector $\left(X^{1}, \ldots, X^{m}\right)$ of stochastic processes is independent.

Now we come to the proof of the converse of Theorem A.1.1, i.e., of the fact that if $X^{1}, \ldots, X^{m}$ are semimartingales and additive processes relative to $\mathbb{F}$ such that the vector $\left(X^{1}, \ldots, X^{m}\right)$ is independent, then $\left[X^{j}, X^{k}\right]=0$ for every $j, k=1, \ldots, m$, $j \neq k$. The proof which we know makes use of the generalization of the Itô-Lévy decomposition to additive processes but in Chapter 2 we have introduced the Itô ${ }^{-}$ Lévy decomposition only for Lévy processes. To avoid technical complications, we do not show the converse of Theorem A.1.1 in the general case of additive processes. Rather, we restrict our attention to the simpler case of Lévy processes. The ideas of the proof are exactly the same in both the cases of Lévy processes and additive processes. We are going to verify the following theorem. 
A.1.7 Theorem. Let $L^{1}, L^{2}$ be Lévy processes relative to $\mathbb{F}$. If the vector $\left(L^{1}, L^{2}\right)$ is independent, then $\left[L^{1}, L^{2}\right]=0$.

We need a general lemma for which we refer to Revuz \& Yor (1999), Chapter IV $\S 1$.

A.1.8 Lemma. Let $M$ be a continuous local martingale with respect to $\mathbb{F}$ and $\mathbb{G}$, where $\mathbb{G}$ is another filtration satisfying the usual conditions. Then

$$
\langle M, M\rangle^{\mathbb{F}}=\langle M, M\rangle^{\mathbb{G}},
$$

where $\langle M, M\rangle^{\mathbb{F}}$ and $\langle M, M\rangle^{\mathbb{G}}$ denote the point brackets of $M$ as an $\mathbb{F}$-martingale and as a $\mathbb{G}$-martingale, respectively.

Now we show that two continuous local martingale which are independent are also orthogonal.

A.1.9 Lemma. Let $M, N \in \mathscr{M}_{\mathrm{loc}, 0}^{c}$ be independent. Then $\langle M, N\rangle=0$.

Proof. To show $\langle M, N\rangle=0$ is equivalent to verify that $M N \in \mathscr{M}_{\text {loc }}$. Let $\mathbb{F}^{M, N}$ be the smallest filtration satisfying the usual conditions and containing the filtration generated by $M$ and $N$. Then $M$ and $N$ are also $\mathbb{F}^{M, N}$-continuous local martingales and because of Lemma A.1.8 we have $\langle M, N\rangle=0$ if and only if $\langle M, N\rangle^{\mathbb{F}^{M, N}}=0$, where $\langle M, N\rangle$ denotes the point brackets of $M$ and $N$ as $\mathbb{F}$-local martingales while $\langle M, N\rangle^{\mathbb{F}^{M, N}}$ denotes the point brackets of $M$ and $N$ as $\mathbb{F}^{M, N}$-local martingales, respectively. This means that we can restrict our attention to the filtration $\mathbb{F}^{M, N}$. We assume in a first step that $M$ and $N$ are uniformly integrable continuous martingales, that is, $M, N \in \mathscr{M}_{0}^{c}\left(\mathbb{F}^{M, N}\right)$. First of all we observe that the independence of $M$ and $N$ implies that the random variable $M_{t} N_{t}$ is integrable, for every $t \geq 0$. We show that $M N$ is a martingale as an application of the theorem on Dynkin systems (cf. Bauer (2001), Chapter I§2). We introduce the system $\mathscr{D}$ by

$$
\mathscr{D}:=\left\{A \in \mathscr{F}_{s}^{M, N}: \mathbb{E}\left[1_{A} M_{t} N_{t}\right]=\mathbb{E}\left[1_{A} M_{s} N_{s}\right]\right\}
$$

and we show that it is a Dynkin system. By the independence of $M$ and $N$, we have that $M_{t}$ and $N_{t}$ are independent for every $t$ and so

$$
\mathbb{E}\left[M_{t} N_{t}\right]=\mathbb{E}\left[M_{t}\right] \mathbb{E}\left[N_{t}\right]=\mathbb{E}\left[M_{s}\right] \mathbb{E}\left[N_{s}\right]=\mathbb{E}\left[M_{s} N_{s}\right],
$$

meaning that $\Omega \in \mathscr{D}$. If $A \in \mathscr{D}$, then $A^{c} \in \mathscr{D}$. Indeed,

$$
\begin{aligned}
\mathbb{E}\left[M_{t} N_{t} 1_{A^{c}}\right] & =\mathbb{E}\left[M_{t} N_{t}\left(1-1_{A}\right)\right]=\mathbb{E}\left[M_{t} N_{t}\right]-\mathbb{E}\left[M_{t} N_{t} 1_{A}\right] \\
& =\mathbb{E}\left[M_{s} N_{s}\right]-\mathbb{E}\left[M_{s} N_{s} 1_{A}\right]=\mathbb{E}\left[M_{s} N_{s}\left(1-1_{A}\right)\right] \\
& =\mathbb{E}\left[M_{s} N_{s} 1_{A^{c}}\right] .
\end{aligned}
$$

It is easy to verify that if $\left(D_{n}\right)_{n \geq 1} \subseteq \mathscr{D}$ is a sequence of pairwise-disjoint subsets, then $\cup_{n=1}^{\infty} D_{n} \in \mathscr{D}$. Therefore $\mathscr{D}$ is a Dynkin system. The system $\mathscr{C}$ defined by

$$
\mathscr{C}:=\left\{A \cap B: A \in \mathscr{F}_{s}^{M}, \quad B \in \mathscr{F}_{s}^{N}\right\}
$$


generates $\mathscr{F}_{s}^{M, N}$ and moreover

$$
\begin{aligned}
\mathbb{E}\left[M_{t} N_{t} 1_{A \cap B}\right] & =\mathbb{E}\left[\left(M_{t} 1_{A}\right)\left(N_{t} 1_{B}\right)\right]=\mathbb{E}\left[M_{t} 1_{A}\right] \mathbb{E}\left[N_{t} 1_{B}\right] \\
& =\mathbb{E}\left[M_{s} 1_{A}\right] \mathbb{E}\left[N_{s} 1_{B}\right]=\mathbb{E}\left[M_{s} N_{s} 1_{A \cap B}\right]
\end{aligned}
$$

This shows $\mathscr{C} \subseteq \mathscr{D}$. An application of the theorem on Dynkin systems yields $\mathscr{D}=$ $\mathscr{F}^{M, N}$. Therefore $M N$ is an $\mathbb{F}^{M, N}$-continuous martingale and hence $\langle M, N\rangle^{\mathbb{F}^{M, N}}=0$ (cf. $\S 1.2 .5$ ). By Lemma A.1.8 we get $\langle M, N\rangle=0$. We now consider the general case of continuous local martingales. Observe that if $Y$ belongs to $\mathscr{M}_{\text {loc, }, 0}^{c}$, then the sequence $\left(T_{n}^{Y}\right)_{n \geq 1}$ of stopping times defined by $T_{n}^{Y}:=\inf \left\{t>0:\left|Y_{t}\right|>n\right\}$ localizes $Y$ to $\mathscr{M}_{0}^{c}$. Indeed, $Y^{T_{n}^{Y}}$ is a bounded local martingale and hence a uniformly integrable martingale. Moreover, the process $Y^{T_{n}^{Y}}$ is $\mathbb{F}^{Y}$-adapted, because $T_{n}^{Y}$ is also an $\mathbb{F}^{Y}$ stopping time, for every $n \geq 1$. Then introducing the stopping times $T_{n}^{M}$ and $T_{n}^{N}$, we have that the uniformly integrable martingales $M^{T_{n}^{M}}$ and $N^{T_{n}^{N}}$ are again independent. From the previous step they are orthogonal. Hence

$$
\langle M, N\rangle=\lim _{n \rightarrow \infty}\langle M, N\rangle^{T_{n}^{M} \wedge T_{n}^{N}}=\lim _{n \rightarrow \infty}\left\langle M^{T_{n}^{M}}, N^{T_{n}^{N}}\right\rangle=0 .
$$

Let $L^{1}$ and $L^{2}$ be two Lévy processes relative to the filtration $\mathbb{F}$ and let $\mathrm{M}^{1}$ and $\mathrm{M}^{2}$ denote the Poisson random measure of $L^{1}$ and $L^{2}$, respectively. The random measure $\mathrm{M}^{j}$ is a measurable functional (on the Skorohod space) of the Lévy process $L^{j}$ and therefore, if $L^{1}$ is independent of $L^{2}$, we also have that $\mathrm{M}^{1}$ is independent of $\mathrm{M}^{2}$.

A.1.10 Lemma. Let $\left(L^{1}, \mathbb{F}\right)$ and $\left(L^{2}, \mathbb{F}\right)$ be independent Lévy processes with Lévy measure $\nu^{1}$ and $\nu^{2}$, respectively. Then the Lévy processes $\mathrm{M}^{1}\left(1_{\left[0, \cdot \times\left\{|x|>\frac{1}{n}\right\}\right.} x\right)$ and $\mathrm{M}^{2}\left(1_{[0, \cdot] \times\left\{|x|>\frac{1}{n}\right\}} x\right)$ have no common jumps.

Proof. The process $\mathrm{M}^{j}\left(1_{[0, \cdot] \times\left\{|x|>\frac{1}{n}\right\}} x\right)$ is $\mathbb{F}^{\mathrm{M}^{j}}$-adapted (cf. Proposition 2.3.10), therefore we obtain that $\mathrm{M}^{1}\left(1_{[0, \cdot] \times\left\{|x|>\frac{1}{n}\right\}} x\right)$ and $\mathrm{M}^{2}\left(1_{[0, \cdot] \times\left\{|x|>\frac{1}{n}\right\}} x\right)$ are independent Lévy processes. Moreover, they are both compound Poisson processes. Let $T_{1}^{j}$ be the first jump-time of $\mathrm{M}^{j}\left(1_{[0, \cdot] \times\left\{|x|>\frac{1}{n}\right\}} x\right)$, i.e., $T_{1}^{j}:=\inf \left\{t>0:\left|\Delta \mathrm{M}^{j}\left(1_{[0, t] \times\left\{|x|>\frac{1}{n}\right\}} x\right)\right|>0\right\}$. We define recursively the sequence of the jump times of $\mathrm{M}^{j}\left(1_{[0,] \times\left\{|x|>\frac{1}{n}\right\}} x\right)$ by

$$
T_{q+1}^{j}:=\inf \left\{t>T_{q}^{j}:\left|\Delta \mathrm{M}^{j}\left(1_{[0, t] \times\left\{|x|>\frac{1}{n}\right\}} x\right)\right|>0\right\}, \quad q \in \mathbb{N} .
$$

The processes $\mathrm{M}^{j}\left(1_{\left[0, \cdot \times\left\{|x|>\frac{1}{n}\right\}\right.} x\right)$ are stochastically continuous and so, for every fixed $t \geq 0$, we have that the set $\left\{\Delta \mathrm{M}^{j}\left(1_{[0, t] \times\left\{|x|>\frac{1}{n}\right\}} x\right) \mid>0\right\}$ is a null set. Therefore every event of the kind $\left\{T_{q}^{j}=s\right\} \subseteq\left\{\Delta \mathrm{M}^{j}\left(1_{[0, t] \times\left\{|x|>\frac{1}{n}\right\}} x\right) \mid>0\right\}, s>0$, has probability equal to zero. Hence

$$
\mathbb{P}\left[T_{q}^{1}=T_{p}^{2}\right]=\int_{0}^{+\infty} \mathbb{P}\left[T_{q}^{1}=T_{p}^{2} \mid T_{p}^{2}=s\right] d \mathbb{P}_{T_{p}^{2}}=\int_{0}^{+\infty} \mathbb{P}\left[T_{q}^{1}=s\right] d \mathbb{P}_{T_{p}^{2}}=0,
$$


where by $\mathbb{P}_{T_{p}^{2}}$ we denoted the law of $T_{p}^{2}$ and we used the independence to get the second equality and the stochastic continuity to get the third one. This means that the two processes $\mathrm{M}^{1}\left(1_{[0, \cdot] \times\left\{|x|>\frac{1}{n}\right\}} x\right)$ and $\mathrm{M}^{2}\left(1_{[0, \cdot] \times\left\{|x|>\frac{1}{n}\right\}} x\right)$ have no common jumps and the proof is concluded.

We are now ready to prove Theorem A.1.7.

Proof of Theorem A.1.7. In a first step we assume that $L^{1}$ and $L^{2}$ are purely nonGaussian Lévy processes with no drift part. Then for $L^{j}$ we have the following Itô-Lévy decomposition

$$
L^{j}=\mathrm{M}^{j}\left(1_{[0, \cdot \times\{|x|>1\}} x\right)+\overline{\mathrm{M}}^{j}\left(1_{[0, \cdot \times\{|x| \leq 1\}} x\right), \quad t \geq 0, \quad j=1,2 .
$$

The processes $L^{j, n}, j=1,2$, defined by

$$
L_{t}^{j, n}:=\sum_{0<s \leq t} 1_{\left\{\frac{1}{n}<\left|\Delta L_{s}^{j}\right| \leq 1\right\}} \Delta L_{s}^{j}-t \int_{\left\{\frac{1}{n}<|x| \leq 1\right\}} x \mathrm{~d} \nu^{j}, \quad t \geq 0, \quad j=1,2,
$$

are martingales and Lévy processes. Moreover, $L^{1, n}$ and $L^{2, n}$ are independent and of finite variation. From Lemma A.1.10, we get

$$
\Delta L^{1, n} \Delta L^{2, n}=0, \quad t \geq 0, \quad \text { a.s. }, \quad n \geq 1 .
$$

Because of Doob's inequality and the isometry, for every $T>0$, we get

$$
\begin{gathered}
\mathbb{E}\left[\sup _{t \leq T}\left(\overline{\mathrm{M}}^{j}\left(1_{[0, t] \times\{|x| \leq 1\}} x\right)-L_{t}^{j, n}\right)^{2}\right] \leq 4 \mathbb{E}\left[\left(\overline{\mathrm{M}}^{j}\left(1_{[0, T] \times\{|x| \leq 1\}} x\right)-L_{T}^{j, n}\right)^{2}\right] \\
=4 T\left\|\left(1_{\{|x| \leq 1\}}-1_{\left\{\frac{1}{n}<|x| \leq 1\right\}}\right)\right\|_{L^{2}\left(\nu^{j}\right)}^{2} \longrightarrow 0 \quad \text { as } n \rightarrow+\infty .
\end{gathered}
$$

Therefore, for every $t \leq T$ and a.s., it follows

$$
\Delta \overline{\mathrm{M}}^{1}\left(1_{[0, t] \times\{|x| \leq 1\}} x\right) \Delta \overline{\mathrm{M}}^{2}\left(1_{[0, t] \times\{|x| \leq 1\}} x\right)=\lim _{n \rightarrow \infty} \Delta L^{1, n} \Delta L^{2, n}=0
$$

and because of the arbitrariness of $T>0$,

$$
\Delta \overline{\mathrm{M}}^{1}\left(1_{[0, \cdot \times\{|x| \leq 1\}} x\right) \Delta \overline{\mathrm{M}}^{2}\left(1_{[0, \cdot] \times\{|x| \leq 1\}} x\right)=0
$$

Similarly, one obtains $\Delta \mathrm{M}^{j}\left(1_{[0, \cdot] \times\{|x|>1\}} x\right) \Delta \overline{\mathrm{M}}^{k}\left(1_{[0, \cdot] \times\{|x| \leq 1\}} x\right)=0$ for $j, k=1,2$, $j \neq k$. We now consider the general case. We have

$$
L_{t}^{j}=\beta^{j} t+\mathrm{W}_{t}^{\sigma^{j}}+\mathrm{M}^{j}\left(1_{[0, t] \times\{|x|>1\}} x\right)+\overline{\mathrm{M}}^{j}\left(1_{[0, t] \times\{|x| \leq 1\}} x\right), \quad t \geq 0, \quad j=1,2 .
$$

By the independence of $L^{1}$ and $L^{2}$ we have that $\left(L^{1}, \mathrm{M}^{1}\right)$ is independent of $\left(L^{2}, \mathrm{M}^{2}\right)$. Hence the vector $\left(L^{1}, \mathrm{M}^{1}\left(1_{[0,] \times\{|x|>1\}} x\right), \overline{\mathrm{M}}^{1}\left(1_{[0, \cdot] \times\{|x| \leq 1\}} x\right)\right)$ is independent of the vector $\left(L^{2}, \mathrm{M}^{2}\left(1_{[0, \cdot] \times\{|x|>1\}} x\right), \overline{\mathrm{M}}^{2}\left(1_{[0, \cdot] \times\{|x| \leq 1\}} x\right)\right)$. Because of the Itô-Lévy decomposition we can conclude that $\mathrm{W}^{\sigma^{1}}$ is independent of $\mathrm{W}^{\sigma^{2}}$. Applying Lemma A.1.9, we have $\left\langle\mathrm{W}^{\sigma^{1}}, \mathrm{~W}^{\sigma^{2}}\right\rangle=0$. If we introduce the process $Y^{j}$ by $Y_{t}^{j}:=L_{t}^{j}-\beta_{t}^{j}-\mathrm{W}^{\sigma^{j}}$, 
$j=1,2$, by the previous step we have that $Y^{1}$ and $Y^{2}$ do not have common jumps, i.e., $\Delta Y^{1} \Delta Y^{2}=0$. On the other side, $\left(L^{j}\right)^{c}=\mathrm{W}^{\sigma^{j}}$ and so

$$
\begin{aligned}
{\left[L^{1}, L^{2}\right] } & =\left\langle\left(L^{1}\right)^{c},\left(L^{2}\right)^{c}\right\rangle+\sum_{0<s \leq \cdot} \Delta L_{s}^{1} \Delta L_{s}^{2} \\
& =\left\langle\mathrm{W}^{\sigma^{1}}, \mathrm{~W}^{\sigma^{2}}\right\rangle+\sum_{0<s \leq .} \Delta Y_{s}^{1} \Delta Y_{s}^{2}=0 .
\end{aligned}
$$

We conclude with an interesting corollary to Theorem A.1.7.

A.1.11 Corollary. Let $L^{1}, \ldots, L^{m}$ be Lévy processes relative to $\mathbb{F}$.

(i) The vector of stochastic processes $\left(L^{1}, \ldots, L^{m}\right)$ is independent if and only if the vectors $\left(L^{j}, L^{k}\right)$ are independent, for every $j, k=1, \ldots, m, j \neq k$.

(ii) If the vectors $\left(L^{j}, L^{k}\right)$ are independent, for every $j, k=1, \ldots, m, j \neq k$, then the vector of stochastic processes $\left(L^{1}, \ldots, L^{m}\right)$ has $\mathbb{F}$-independent increments.

Proof. (i) It is clear that if the vector $\left(L^{1}, \ldots, L^{m}\right)$ is independent, then we have also pairwise independence. On the contrary, if $\left(L^{j}, L^{k}\right)$ are independent, $j, k=1, \ldots, m$, $j \neq k$, because of Theorem A.1.7, we also have that $\left[L^{j}, L^{k}\right]=0, j, k=1, \ldots, n$, $j \neq k$. Applying Theorem A.1.1 we get that $\left(L^{1}, \ldots, L^{m}\right)$ is jointly independent.

(ii) If the vectors $\left(L^{j}, L^{k}\right)$ are independent, for every $j, k=1, \ldots, m, j \neq k$, Theorem A.1.7 implies that $\left[L^{j}, L^{k}\right]=0, j, k=1, \ldots, m, j \neq k$. But then, from Theorem A.1.2, we get that the vector $\left(L^{1}, \ldots, L^{m}\right)$ has $\mathbb{F}$-independent increments, and the proof is finished.

An analogue of Corollary A.1.11 can be formulated also for processes which are semimartingales and additive processes relative to the filtration $\mathbb{F}$, provided that Theorem A.1.7 has been generalized to this case.

\section{A.2. Itô-Lévy Decomposition}

We shall prove in this appendix the Itô-Lévy decomposition. We are going to state this result for Lévy processes relative to a filtration. We need some preparation.

In $\S 2.2 .5$, we have shown that if $X$ is a càdlàg adapted process whose jump measure $\mathrm{M}$ is a Poisson random measure with respect to the filtration $\mathbb{F}$ with intensity measure $\lambda_{+} \otimes \nu$, where $\lambda_{+}$is the Lebesgue measure on $\left(\mathbb{R}_{+}, \mathscr{B}\left(\mathbb{R}_{+}\right)\right)$and $\nu$ a Lévy measure on $(\mathbb{R}, \mathscr{B}(\mathbb{R}))$, then for every $f$ such that $|f| \wedge 1 \in L^{1}(\nu)$, the process $\left(\mathrm{M}\left(1_{[0,]} f\right), \mathbb{F}\right)$ is a Lévy process.

Now we assume that $(L, \mathbb{F})$ is a Lévy process. Under this assumption, we know that the jump measure $\mathrm{M}$ of $L$ is a Poisson random measure relative to the filtration $\mathbb{F}$ with intensity measure $\lambda_{+} \otimes \nu$, where $\nu$ is the Lévy measure of the process $L$ (cf. Theorem 2.3.4). We recall that we denoted by $\left(\mathscr{D}, \mathscr{F}_{D}\right)$ the Skorohod space over $\mathbb{R}$. We are going to show that the increments of the process $\mathrm{M}\left(1_{[0, \cdot]} f\right)$, where $f$ is such that $|f| \wedge 1 \in L^{1}(\nu)$, can be represented as a time-homogeneous $\left(\mathscr{D}, \mathscr{F}_{D}\right)$-measurable of the increments of $L$. In other words, our aim is to extend Lemma 2.3.1 to any 
deterministic function $f$ such that $|f| \wedge 1 \in L^{1}(\nu)$. By ${ }^{t} L$ we denoted the process defined by

$$
{ }^{t} L_{s}:=L_{t+s}-L_{t}, \quad s, t \geq 0 .
$$

while $\mathscr{D}$ denotes the system of simple functions in $L^{q}(\nu)$, for $q \in[1,+\infty]$, that is,

$$
\mathscr{D}:=\left\{f=\sum_{j=1}^{m} a_{j} 1_{C_{j}}, \quad a_{j} \in \mathbb{R}, \quad C_{j} \in \mathscr{B}(\mathbb{R}): \quad \nu\left(C_{j}\right)<+\infty\right\} .
$$

A.2.1 Lemma. Let $f \in \mathscr{D}, f \geq 0$. Then the increments of the process $\mathrm{M}\left(1_{[0, \cdot]} f\right)=$ $\left(\mathrm{M}\left(1_{[0, t]} f\right)\right)_{t \geq 0}$ can be represented as

$$
\mathrm{M}\left(1_{(t, t+s]} f\right)=F_{s, f}\left({ }^{t} L\right), \quad \text { a.s., } \quad t, s \geq 0,
$$

where $F_{s, f}$ is a measurable functional on the Skorohod space $\left(D, \mathscr{F}_{D}\right)$ and ${ }^{t} L$ is the process defined in (A.9).

Proof. Let $f \in \mathscr{D}, f \geq 0$, with representation $f=\sum_{j=1}^{m} a_{j} 1_{C_{j}}$, where $a_{j} \geq 0$ for every $j=1, \ldots, m$. For each $C_{j}$ appearing in the representation of $f$, we introduce the sequence $B_{j}^{n}:=C_{j} \cap\left\{|x|>\frac{1}{n}\right\}$ and consequently the function

$$
f_{n}:=\sum_{j=1}^{m} a_{j} 1_{B_{j}^{n}}, \quad n \geq 1
$$

The process $\xi^{B_{j}^{n}}$ is given in (2.30). Because of Lemma 2.3.1, we have

$$
\mathrm{M}\left(1_{(t, t+s]} f_{n}\right)=\sum_{j=1}^{m} a_{j}\left(\xi_{t+s}^{B_{j}^{n}}-\xi_{t}^{B_{j}^{n}}\right)=\sum_{j=1}^{m} a_{j} F_{s, B_{j}^{n}}\left({ }^{t} L\right), \quad \text { a.s. }, \quad t, s \geq 0, \quad n \geq 1,
$$

where $F_{s, B_{j}^{n}}(\cdot)$ is a measurable functional on the Skorohod space $\left(D, \mathscr{F}_{D}\right)$ and ${ }^{t} L$ is the process defined in (A.9). For every $t \geq 0$ we have $\mathrm{M}\left(1_{[0, t]} f_{n}\right) \uparrow \mathrm{M}\left(1_{[0, t]} f\right)$ pointwise in $\omega$ as $n \rightarrow+\infty$. Therefore, if we put

$$
F_{s, f}\left({ }^{t} L\right):=\liminf _{n \rightarrow+\infty} \sum_{j=1}^{m} a_{j} F_{s, B_{j}^{n}}\left({ }^{t} L\right)
$$

we have $\mathrm{M}\left(1_{(t, t+s]} f\right)=F_{s, f}\left({ }^{t} L\right)$ a.s. for every $t, s \geq 0, f \in \mathscr{D}, f \geq 0$.

We observe that, from Lemma 1.1.8, the system $\mathscr{D} \subseteq L^{q}(\nu)$ of simple functions is dense in $L^{q}(\nu)$. As a consequence of this fact we can extend Lemma A.2.1.

A.2.2 Proposition. Let $f$ be such that $|f| \wedge 1 \in L^{1}(\nu)$. The increments of the process $\mathrm{M}\left(1_{[0, \cdot]} f\right)=\left(\mathrm{M}\left(1_{[0, t]} f\right)\right)_{t \geq 0}$ can be represented as

$$
\mathrm{M}\left(1_{(t, t+s]} f\right)=F_{s, f}\left({ }^{t} L\right), \quad \text { a.s., } \quad t, s \geq 0,
$$

where $F_{s, f}$ is a measurable functional on the Skorohod space $\left(D, \mathscr{F}_{D}\right)$ and ${ }^{t} L$ is the process defined in (A.9). 
Proof. First we consider the case $f \in L^{1}(\nu), f \geq 0$. The set $\mathscr{D}$ of simple functions is dense in $L^{1}(\nu)$ and so there exists a sequence $\left(f_{n}\right)_{n \in \mathbb{N}} \subseteq \mathscr{D}$ such that $f_{n} \geq 0, n \geq 1$, and $f_{n} \uparrow f$ in $L^{1}(\nu)$ as $n \rightarrow+\infty$. By monotone convergence, $\mathrm{M}\left(1_{[0, t]} f_{n}\right) \uparrow \mathrm{M}\left(1_{[0, t]} f\right)$ pointwise in $\omega$ as $n \rightarrow+\infty$. By Lemma A.2.1, we have

$$
\mathrm{M}\left(1_{(t, t+s]} f_{n}\right)=F_{s, f_{n}}\left({ }^{t} L\right), \quad \text { a.s., } \quad s, t \geq 0, \quad n \geq 1,
$$

where $F_{s, f_{n}}$ is a measurable functional on the Skorohod space $\left(D, \mathscr{F}_{D}\right)$. If we put

$$
F_{s, f}\left({ }^{t} L\right):=\liminf _{n \rightarrow+\infty} F_{s, f_{n}}\left({ }^{t} L\right)
$$

we obtain, $\mathrm{M}\left(1_{(t, t+s]} f\right)=F_{s, f_{n}}\left({ }^{t} L\right)$ and $F_{s, f}$ is a measurable functional on the Skorohod space $\left(D, \mathscr{F}_{D}\right)$. We now weaken the assumptions and consider $f$ such that $|f| \wedge 1 \in L^{1}(\nu)$. Because of Proposition 2.2.7, the stochastic integral $\mathrm{M}\left(1_{[0, t]} f\right)$ exists and it is finite a.s. for every $t \geq 0$. If $f^{+}$and $f^{-}$are the positive and the negative part of $f$, respectively, we have $f^{ \pm} \leq|f|$ so that $f^{ \pm} \wedge 1 \in L^{1}(\nu)$ and the stochastic integrals $\mathrm{M}\left(1_{[0, t]} f^{ \pm}\right)$are well defined and the relation $\mathrm{M}\left(1_{[0, t]} f\right)=$ $\mathrm{M}\left(1_{[0, t]} f^{+}\right)-\mathrm{M}\left(1_{[0, t]} f^{-}\right)$holds. Let us introduce the functions $f_{n}^{ \pm}:=f^{ \pm} 1_{\left\{\left|f^{ \pm}\right|<n\right\}}$ and $f_{n}:=f_{n}^{+}-f_{n}^{-}$. Then $f_{n}^{ \pm} \uparrow f^{ \pm}$and $f_{n}^{ \pm} \geq 0$. By monotone convergence we get $\mathrm{M}\left(1_{[0, t]} f_{n}^{ \pm}\right) \uparrow \mathrm{M}\left(1_{[0, t]} f^{ \pm}\right)$pointwise in $\omega$ as $n \rightarrow+\infty$. Furthermore, $f_{n}^{ \pm} \in L^{1}(\nu)$, indeed $f_{n}^{ \pm} \leq\left(f^{ \pm} \wedge n\right) \leq n\left(f^{ \pm} \wedge 1\right) \in L^{1}(\nu)$, for every $n \geq 1$. Because of the previous step, $\mathrm{M}\left(1_{(t, t+s]} f_{n}^{ \pm}\right)=F_{s, f_{n}}\left({ }^{t} L^{ \pm}\right)$a.s., where $F_{s, f_{n}^{ \pm}}$is a measurable functional on the Skorohod space $\left(D, \mathscr{F}_{D}\right)$. We can put

$$
F_{s, f^{ \pm}}\left({ }^{t} L\right):=\liminf _{n \rightarrow+\infty} F_{s, f_{n}}\left({ }^{t} L^{ \pm}\right)
$$

which is a measurable functional on the Skorohod space $\left(D, \mathscr{F}_{D}\right)$. Hence it follows that $\mathrm{M}\left(1_{(t, t+s]} f^{ \pm}\right)=F_{s, f^{ \pm}}\left({ }^{t} L\right)$ a.s. and from $\mathrm{M}\left(1_{[0, t]} f\right)=\mathrm{M}\left(1_{[0, t]} f^{+}\right)-\mathrm{M}\left(1_{[0, t]} f^{-}\right)$ we have

$$
\mathrm{M}\left(1_{(t, t+s]} f\right)=F_{s, f^{+}}\left({ }^{t} L\right)-F_{s, f^{-}}\left({ }^{t} L\right)=: F_{s, f}\left({ }^{t} L\right), \quad \text { a.s., } \quad s, t \geq 0 .
$$

In conclusion, we have shown that for every function $f$ such that $|f| \wedge 1 \in L^{1}(\nu)$ the increments of the process $\mathrm{M}\left(1_{[0, \cdot]} f\right)$ can be represented as a homogeneous functional of ${ }^{t} L$ which is measurable on the Skorohod space $\left(D, \mathscr{F}_{D}\right)$.

Let $(L, \mathbb{F})$ be a Lévy process and let $\mathrm{M}$ be its jump measure. From $§ 2.3 .1$, we know that $\mathrm{M}$ is a Poisson random measure relative to $\mathbb{F}$ and that its intensity measure is $\lambda_{+} \otimes \nu$, where $\lambda_{+}$is the Lebesgue measure on $\mathbb{R}_{+}$and $\nu$ the Lévy measure of $L$. We define $f(x):=1_{\{|x|>1\}} x$. The function $f$ is such that $|f| \wedge 1 \in L^{1}(\nu)$. Clearly this implies that the function $h(t, x):=1_{[0, t]} f$ is such that $|h| \wedge 1 \in L^{1}\left(\lambda_{+} \otimes \nu\right)$. From Proposition 2.2.7, we can consider the stochastic integral of $h$ with respect to M. We introduce the process $L^{2}=\left(L_{t}^{2}\right)_{t \geq 0}$ by

$$
L_{t}^{2}:=\mathrm{M}\left(1_{[0, t]} f\right):=\mathrm{M}\left(1_{[0, t] \times\{|x|>1\}} x\right), \quad t \geq 0 .
$$

By Proposition 2.2.15, we know that $\left(L^{2}, \mathbb{F}\right)$ is a Lévy process and furthermore, from Proposition A.2.2, that its increments can be written as it follows:

$$
L_{t+s}^{2}-L_{t}^{2}=F_{s, f}\left({ }^{t} L\right), \quad \text { a.s. }, \quad t, s \geq 0,
$$


where $F_{\cdot, f}\left({ }^{t} L\right)$ is an $\left(D, \mathscr{F}_{D}\right)$-measurable homogeneous functional of the process ${ }^{t} L$. Let now $Y=\left(Y_{t}\right)_{t \geq 0}$ be the process defined by

$$
Y_{t}:=L_{t}-L_{t}^{2}, \quad t \geq 0 .
$$

Clearly, $(Y, \mathbb{F})$ is a càdlàg process starting at 0 and its increments are $\mathbb{F}$-independent and homogeneous, because $Y_{t+s}-Y_{t}={ }^{t} L_{s}-F_{s, f}\left({ }^{t} L\right)$ a.s., $s, t \geq 0$, which is clearly a measurable functional on the Skorohod space of the increment $L_{t+s}-L_{s}$. These properties imply that $Y$ is also stochastically continuous and therefore $(Y, \mathbb{F})$ is a Lévy process. Notice that the relation

$$
L_{t}^{2}=\sum_{0<s \leq t} 1_{\left\{\left|\Delta L_{s}\right|>1\right\}} \Delta L_{s}, \quad t \geq 0, \quad \text { a.s. }
$$

holds. Therefore $L_{t}^{2}$ is the sum of all the jumps of the process $L$ up to time $t$ which are bigger than one. This implies that the process $Y$ has only jumps of size smaller or equal than one. Consequently, $(Y, \mathbb{F})$ is a Lévy process with bounded jumps and, because of Proposition 2.1.6, it has a finite moment of every order. In particular, $\mathbb{E}\left[Y_{t}^{2}\right]<+\infty$ for every $t \geq 0$. Hence the Lévy process $\bar{Y}=\left(\bar{Y}_{t}\right)_{t \geq 0}$ defined by

$$
\bar{Y}_{t}:=Y_{t}-\mathbb{E}\left[Y_{t}\right], \quad t \geq 0
$$

is an $\mathbb{F}$-martingale (cf. Lemma 2.1.1). Because of the square integrability of $\bar{Y}_{t}, \geq 0$, an application of Doob's inequality shows that, for every arbitrary but fixed $T>0$, the process $(\bar{Y})_{0 \leq t \leq T}$ is uniformly integrable. Consequently, the process $(Y)_{0 \leq t \leq T}$ is uniformly integrable. An application of the generalization of theorem of Lebesgue on dominated convergence to uniformly integrable families of random variables (cf. Meyer (1966), Theorem II.21) shows that the mapping $t \mapsto \mathbb{E}\left[Y_{t}\right]$ is continuous. We now define $a(t):=\mathbb{E}\left[Y_{t}\right], t \geq 0$. Because of the homogeneity of the increments we have

$$
a(t+s)=\mathbb{E}\left[Y_{t+s}-Y_{t}\right]+\mathbb{E}\left[Y_{t}\right]=a(t)+a(s), \quad s, t \geq 0 .
$$

We know that $a(\cdot)$ is continuous and from the previous relation it follows that $a(\cdot)$ is a linear function. Therefore there exists $\beta \in \mathbb{R}$ such that

$$
a(t)=\beta t, \quad t \geq 0 .
$$

Now we are ready to prove the Itô-Lévy decomposition.

A.2.3 Theorem (Itô-Lévy decomposition). Let $L$ be a càdlàg adapted process with jump measure $\mathrm{M}$. Then $(L, \mathbb{F})$ is a Lévy process if and only if $\mathrm{M}$ is a Poisson random measure relative to $\mathbb{F}$ with intensity function $\lambda_{+} \otimes \nu$, where $\lambda_{+}$is the Lebesgue measure on $\mathbb{R}_{+}$and $\nu$ a Lévy measure, and there exists a Wiener process $\left(\mathrm{W}^{\sigma}, \mathbb{F}\right)$ with variance function $\sigma^{2}(t)=\sigma^{2} t, \sigma^{2} \geq 0$, called Gaussian part of $L$, such that the following decomposition holds

$$
L_{t}=\beta t+\mathrm{W}_{t}^{\sigma}+\mathrm{M}\left(1_{[0, t] \times\{|x|>1\}} x\right)+\overline{\mathrm{M}}\left(1_{[0, t] \times\{|x| \leq 1\}} x\right), \quad t \geq 0, \text { a.s. }
$$

where $\beta \in \mathbb{R}$. 
Proof. We assume first that $(L, \mathbb{F})$ is a Lévy process. From $\S 2.3 .1$ we know that $\mathrm{M}$ is a Poisson random measure relative to $\mathbb{F}$ and that its intensity measure is $\lambda_{+} \otimes \nu$, where $\lambda_{+}$is the Lebesgue measure on $\mathbb{R}_{+}$and $\nu$ the Lévy measure of $L$. We introduce the function $g(x):=1_{\{|x| \leq 1\}} x$ and the sequence $g^{n}:=1_{\left\{\frac{1}{n}<|x| \leq 1\right\}} x$. The function $g$ belongs to $L^{2}(\nu)$, while $g^{n} \in L^{1}(\nu) \cap L^{2}(\nu), n \geq 1$, and $g^{n} \longrightarrow g$ in $L^{2}(\nu)$ as $n \rightarrow+\infty$. We now define the processes $L^{3}=\left(L_{t}^{3}\right)_{t \geq 0}$ and $L^{3, n}=\left(L_{t}^{3, n}\right)_{t \geq 0}$ by

$$
L_{t}^{3}:=\overline{\mathrm{M}}\left(1_{[0, t]} g\right) ; \quad L_{t}^{3, n}:=\overline{\mathrm{M}}\left(1_{[0, t]} g^{n}\right), \quad t \geq 0, \quad n \geq 1 .
$$

Because of the isometry of the stochastic integral, we have $L_{t}^{3, n} \longrightarrow L_{t}^{3}$ in $L^{2}(\mathbb{P})$ as $n \rightarrow+\infty$, for every $t \geq 0$. By Theorem 2.2 .16 , the process $L^{3}$ is an $\mathbb{F}$-locally square integrable martingale such that $L_{t}^{3}$ is square integrable, for every $t \geq 0$. Moreover, $\left(L^{3}, \mathbb{F}\right)$ is a Lévy process. The same statements holds for the process $\left(L^{3, n}, \mathbb{F}\right)$. Furthermore, from Proposition A.2.2, we know that the increments of $L^{3, n}$ can be represented by a homogeneous $\left(D, \mathscr{F}_{D}\right)$-measurable functional of the process ${ }^{t} L$ (cf. (A.9)). We denote such a functional by $G, g^{n}$. We now introduce the processes $\tilde{L}^{1}=\left(\tilde{L}_{t}^{1}\right)_{t \geq 0}$ and $\tilde{L}^{1, n}=\left(\tilde{L}_{t}^{1, n}\right)_{t \geq 0}$ by

$$
\tilde{L}^{1}:=L-L^{2}-L^{3}=Y-L^{3}, \quad \tilde{L}^{1, n}:=L-L^{2}-L^{3, n}=Y-L^{3, n},
$$

respectively, where the processes $L^{2}$ and $Y$ were introduced by (A.11) and (A.12), respectively. The process $\tilde{L}^{1, n}$ has $\mathbb{F}$-independent and homogeneous increments because

$$
\tilde{L}_{t+s}^{1, n}-\tilde{L}_{s}^{1, n}={ }^{t} L_{s}-F_{s, f}\left({ }^{t} L\right)-G_{s, g^{n}}\left({ }^{t} L\right), \quad s, t \geq 0 .
$$

Furthermore, $\tilde{L}_{t}^{1, n}$ converges in probability to $\tilde{L}_{t}^{1}$, for every $t \geq 0$, as $n \rightarrow+\infty$, because $L_{t}^{3, n}$ converges in $L^{2}(\mathbb{P})$, and hence in probability, to $L_{t}^{3}$, as $n \rightarrow+\infty$, for every $t \geq 0$. Hence Lemma 2.1.2 yields that $\tilde{L}^{1}$ has $\mathbb{F}$-independent and homogeneous increments. From Theorem 2.2.16.(iv), we know that the process $L^{3}$ has the following jumps:

$$
\Delta L^{3}=1_{\{\Delta L \neq 0\}} 1_{\{|\Delta L| \leq 1\}} \Delta L \quad \text { a.s. }
$$

Therefore the process $\left(L^{2}+L^{3}\right)$ has the same jumps of the process $L$ a.s. But then we can claim that the process $\tilde{L}^{1}$ is continuous a.s. The filtration $\mathbb{F}$ satisfies the unusual conditions and so we can find an adapted version of the process $\tilde{L}^{1}$ which is in fact continuous and has $\mathbb{F}$-independent and homogeneous increments. We denote again by $\tilde{L}^{1}$ such a modification. Then the process $\left(\tilde{L}^{1}, \mathbb{F}\right)$ is a continuous Lévy process. The process $Y$ introduced by (A.12) is such that $Y_{t}$ is square integrable, for every $t \geq 0$ and the same holds for $L^{3}$. Moreover, $\mathbb{E}\left[L_{t}^{3}\right]=0$. This implies that $\tilde{L}_{t}^{1}$ is square integrable and that $\mathbb{E}\left[\tilde{L}_{t}^{1}\right]=\mathbb{E}\left[Y_{t}\right]$, for every $t \geq 0$. We have seen that $\mathbb{E}\left[Y_{t}\right]=\beta t, t \geq 0$, where $\beta \in \mathbb{R}$. We introduce the process $L^{1}=\left(L_{t}^{1}\right)_{t \geq 0}$ by

$$
L_{t}^{1}=\tilde{L}_{t}^{1}-\beta t, \quad t \geq 0 .
$$

Clearly $\left(L^{1}, \mathbb{F}\right)$ is a continuous Lévy process and because of $\mathbb{E}\left[L_{t}^{1}\right]=0$, Lemma 2.1.1 implies that it is an $\mathbb{F}$-martingale. Moreover, $L_{t}^{1}$ is square integrable, for every $t \geq 0$. We can assert that $L^{1}$ is a continuous locally square integrable martingale. By Doob's inequality, we get that for every arbitrary but fixed $T>0$ the family 
$\left(\left(L_{t}^{1}\right)^{2}\right)_{0 \leq t \leq T}$ is uniformly integrable. As a consequence of Meyer (1966), Theorem II.21, the mapping $t \mapsto \mathbb{E}\left[\left(L_{t}^{1}\right)^{2}\right]$ is continuous. We put $\sigma^{2}(t):=\mathbb{E}\left[\left(L_{t}^{1}\right)^{2}\right], t \geq 0$. This is a continuous function and moreover, because of the homogeneity and the $\left(\mathbb{F}\right.$-)independence of the increments of $L^{1}$ and of $\mathbb{E}\left[L_{t}^{1}\right]=0, t \geq 0$, we get

$\sigma^{2}(t+s)=\mathbb{E}\left[\left(L_{t+s}^{1}-L_{t}^{1}+L_{t}^{1}\right)^{2}\right]=\mathbb{E}\left[\left(L_{t+s}^{1}-L_{t}^{1}\right)^{2}\right]+\mathbb{E}\left[\left(L_{t}^{1}\right)^{2}\right]=\sigma^{2}(t)+\sigma^{2}(s), \quad t, s \geq 0$.

This relation and the continuity of $\sigma^{2}(\cdot)$, imply that $\sigma^{2}(\cdot)$ is a linear function, i.e., there exists $\sigma^{2} \geq 0$ such that $\sigma^{2}(t)=\sigma^{2} t$, for every $t \geq 0$. In conclusion, we have shown that $\left(L^{1}, \mathbb{F}\right)$ is a continuous Lévy process and a locally square integrable martingale with a linear variance function. We can claim that $\left(L^{1}, \mathbb{F}\right)$ is a Wiener process with variance function $\sigma^{2}$ (cf. Definition 1.2.8). We put $\mathrm{W}^{\sigma}:=L^{1}$ and so from (A.18), we have

$$
\mathrm{W}_{t}^{\sigma}:=\tilde{L}_{t}^{1}-\beta t, \quad t \geq 0 .
$$

Using the definition of $\tilde{L}^{1}$ (cf. (A.17)), we obtain

$$
\mathrm{W}_{t}^{\sigma}=L_{t}-L_{t}^{2}-L_{t}^{3}-\beta t .
$$

Recalling (A.11) and (A.16) this yields (A.15) and this concludes the first part of the proof. Conversely, we now assume that $L$ is a càdlàg process with jump measure M, which is a Poisson random measure with intensity $\lambda_{+} \otimes \nu$, where $\nu$ is a Lévy measure. We assume that $\left(\mathrm{W}^{\sigma}, \mathbb{F}\right)$ is a Wiener process with variance function $\sigma^{2}(t)=\sigma^{2} t, \sigma^{2} \geq 0$, such that (A.15) holds. We verify that $L$ is a Lévy process. We put

$$
\left(L^{1}, L^{2}, L^{3}\right):=\left(\mathrm{W}^{\sigma}, \mathrm{M}\left(1_{[0, \cdot] \times\{|x|>1\}} x\right), \overline{\mathrm{M}}\left(1_{[0, \cdot] \times\{|x| \leq 1\}} x\right)\right) .
$$

By assumption $\mathrm{W}^{\sigma}$ is a Wiener process. Moreover by Proposition $2.2 .15\left(L^{2}, \mathbb{F}\right)$ is a Lévy process and a semimartingale. Analogously we know that $\left(L^{3}, \mathbb{F}\right)$ is a Lévy process and an $\mathbb{F}$-martingale. The processes $L^{2}$ and $L^{3}$ are purely non-Gaussian Lévy processes and they do not have common jumps. Therefore $\left[L^{2}, L^{3}\right]=0$. Because of the continuity of $L^{1}$, we have that $\left[L^{1}, L^{j}\right]=0, j=1,2$. From Theorem 2.1 .8 we have that the vector $\left(L^{1}, L^{2}, L^{3}\right)$ is independent and has $\mathbb{F}$-independent increments. Because of (A.15), we can assert that also the process $L$ has $\mathbb{F}$-independent increments. Moreover, from (A.15) and the independence of the vector $\left(L^{1}, L^{2}, L^{3}\right)$ we have

$$
\begin{aligned}
\mathbb{E}\left[\mathrm{e}^{i u\left(L_{t}-L_{s}\right)}\right] & =\mathrm{e}^{i u \beta(t-s)} \prod_{j=1}^{3} \mathbb{E}\left[\mathrm{e}^{i u\left(L_{t}^{j}-L_{s}^{j}\right)}\right] \\
& =\mathrm{e}^{i u \beta(t-s)} \prod_{j=1}^{3} \mathbb{E}\left[\mathrm{e}^{i u L_{t-s}^{j}}\right] \\
& =\mathbb{E}\left[\mathrm{e}^{i u L_{t-s}}\right], \quad 0 \leq s \leq t, \quad u \in \mathbb{R},
\end{aligned}
$$

where in the last but one equality we used that $L^{j}$ has homogeneous increments, $j=1,2,3$. Then $L$ is a càdlàg adapted process with homogeneous and $\mathbb{F}$-independent increments such that $L_{0}=0$. Hence $L$ is also stochastically continuous. In conclusion $(L, \mathbb{F})$ is a Lévy process and $\nu$ is its Lévy measure. This completes the proof of the theorem.

An immediate but important consequence of the Itô-Lévy decomposition is the Lévy-Kintchine decomposition of the characteristic function of a Lévy process. We formulate the Lévy-Kintchine decomposition as a corollary of Theorem A.2.3. 
A.2.4 Corollary. Let $L$ be a Lévy process with characteristics $\left(\beta, \sigma^{2}, \nu\right)$. Then for every $u \in \mathbb{R}$ and for every $t \geq 0$ we have

$$
\mathbb{E}\left[\mathrm{e}^{i u L_{t}}\right]=\exp \left(\left(i u \beta-\frac{1}{2} u^{2} \sigma^{2}+\int_{\mathbb{R}}\left(\mathrm{e}^{i u x}-1-i u x 1_{\{|x| \leq 1\}}\right) \mathrm{d} \nu\right) t\right)
$$

In particular, $L$ is a semimartingale and $(2.35)$ is a semimartingale decomposition of $L$.

Proof. We put $\varphi_{t}^{L}(u):=\mathbb{E}\left[\mathrm{e}^{i u L_{t}}\right], t \geq 0, u \in \mathbb{R}$. If (A.19) holds, then the mapping $t \mapsto \varphi_{t}^{L}(u)$ is of finite variation for every $u \in \mathbb{R}$. Therefore, from Theorem 2.1.7, $L$ is a semimartingale. Now we show (A.19). We put

$$
\left(L^{1}, L^{2}, L^{3}\right):=\left(\mathrm{W}^{\sigma}, \mathrm{M}\left(1_{[0, \cdot] \times\{|x|>1\}} x\right), \overline{\mathrm{M}}\left(1_{[0, \cdot] \times\{|x| \leq 1\}} x\right)\right) .
$$

The vector $\left(L^{1}, L^{2}, L^{3}\right)$ is independent because $\left[L^{j}, L^{k}\right]=0, j, k=1,2,3, k \neq j$, (cf. Theorem 2.1.8), and so we have

$$
\varphi_{t}^{L}(u)=\mathrm{e}^{i u \beta t} \varphi_{t}^{L^{1}}(u) \varphi_{t}^{L^{2}}(u) \varphi_{t}^{L^{3}}(u), \quad u \in \mathbb{R}, \quad t \geq 0 .
$$

From (1.8) we have $\varphi_{t}^{L^{1}}(u)=\exp \left(-\frac{1}{2} u^{2} \sigma^{2} t\right)$. Moreover, Lemma 2.2.8 implies that

$$
\varphi_{t}^{L^{2}}(u)=\exp \left(t \int_{\{|x|>1\}}\left(\mathrm{e}^{i u x}-1\right) \mathrm{d} \nu\right) .
$$

To compute $\varphi^{L^{3}}(u)$ we have to proceed by approximation. If we define $f_{n}(x):=$ $1_{\left\{\frac{1}{n}<|x| \leq 1\right\}} x$, then we have $f_{n} \in L^{1}(\nu) \cap L^{2}(\nu)$ and $f_{n} \longrightarrow 1_{\{|x| \leq 1\}} x$ in $L^{2}(\nu)$ as $n \rightarrow+\infty$. By Proposition 2.2.12 and Lemma 2.2.8, we have

$$
\mathbb{E}\left[\mathrm{e}^{i u \overline{\mathrm{M}}\left(1_{[0, t]} f_{n}\right)}\right]=\exp \left(i u t \int_{\left\{\frac{1}{n}<|x| \leq 1\right\}} x \mathrm{~d} \nu\right) \exp \left(t \int_{\left\{\frac{1}{n}<|x| \leq 1\right\}}\left(\mathrm{e}^{i u x}-1\right) \mathrm{d} u\right) .
$$

Passing to the limit as $n \rightarrow+\infty$, it follows

$$
\varphi_{t}^{L^{3}}(u)=\exp \left(t \int_{\{|x| \leq 1\}}\left(\mathrm{e}^{i u x}-1-i u x 1_{\{|x| \leq 1\}}\right) \mathrm{d} \nu\right)
$$

and (A.20) implies (A.19). 
APPENDIX B

Denseness of Polynomials

Let $Z=\left(Z_{\alpha}\right)_{\alpha \in I}$ be a family of random variables, where $I$ denotes an arbitrary set of indexes. By $\mathscr{F}^{Z}$ we designate the $\sigma$-algebra generated by $Z=\left(Z_{\alpha}\right)_{\alpha \in I}$. We consider the probability space $\left(\Omega, \mathscr{F}^{Z}, \mathbb{P}\right)$ and the Hilbert space $L^{2}\left(\Omega, \mathscr{F}^{Z}, \mathbb{P}\right)$. By $\mathscr{K}$ we denote the system

$$
\mathscr{K}:=\left\{\prod_{i=1}^{m} Z_{\alpha_{i}}, \quad \alpha_{1}, \ldots, \alpha_{m} \in I, \quad m \in \mathbb{N} \cup\{0\}\right\}
$$

of monomials in $Z=\left(Z_{\alpha}\right)_{\alpha \in I}$. In this appendix we show that under some assumptions on the family $Z$, the system $\mathscr{K}$ is a total system in $L^{2}\left(\Omega, \mathscr{F}^{Z}, \mathbb{P}\right)$. We require the following property for the family $Z$.

B.1 Assumption. For every $Z_{\alpha} \in Z$ there exists a constant $c_{\alpha}>0$ such that

$$
\mathbb{E}\left[\exp \left(c_{\alpha}\left|Z_{\alpha}\right|\right)\right]<+\infty .
$$

In other words, we require that every $Z_{\alpha}$ admits a finite exponential moment.

We analyze the problem of the totality of the system $\mathscr{K}$ only for a probability measure $\mathbb{P}$. It is clear that the result stated below also holds for an arbitrary finite measure $\mu$. By $\mathscr{C}$ we denote the linear hull of cosine functions obtained from $Z$, that is,

$$
\mathscr{C}:=\operatorname{Span}\left(\left\{\cos \left(\sum_{i=1}^{m} u_{i} Z_{\alpha_{i}}\right), \quad \alpha_{i}, \in I, u_{i}, \in \mathbb{R}, i=1, \ldots, m ; m \in \mathbb{N}\right\}\right) .
$$

B.2 Lemma. The system $\mathscr{C}$ introduced in (B.3) is dense in $L^{2}\left(\Omega, \mathscr{F}^{Z}, \mathbb{P}\right)$.

Proof. Clearly, $\mathscr{C}$ is a linear system of bounded functions containing the function $1=1_{\Omega}$ (take $m=1$ and $u=0$ ). Moreover, $\mathscr{C}$ is stable under multiplication. Indeed, for every $u, v \in \mathbb{R}$ and every $Z_{\alpha}, Z_{\beta} \in Z$, with $\alpha, \beta \in I$, the following relation holds:

$$
\cos \left(u Z_{\alpha}\right) \cos \left(v Z_{\beta}\right)=\frac{1}{2}\left(\cos \left(u Z_{\alpha}+v Z_{\beta}\right)+\cos \left(u Z_{\alpha}-v Z_{\beta}\right)\right) .
$$


To apply Lemma 1.1 .8 and conclude that $\mathscr{C}$ is dense in $L^{2}\left(\Omega, \mathscr{F}^{Z}, \mathbb{P}\right)$, we only need to show that $\mathscr{C}$ generates $\mathscr{F}^{Z}$. The system of functions $\{\cos (u x), x \in \mathbb{R}\}$ is separating points, meaning that $\cos (u x)=\cos (u y)$ for every $u \in \mathbb{R}$ implies $x=y$. To see it we proceed by contraposition. We assume that $x \neq y$ under the condition $\cos (u x)=\cos (u y)$ for every $u \in \mathbb{R}$. It follows, $u x=u y+k \pi$ and so, because $x \neq y$, we get $u=\frac{k \pi}{x-y}$. But, every $u$ belonging to $\left(\frac{k \pi}{x-y}, \frac{(k+1) \pi}{x-y}\right)$ yields $\cos (u x) \neq \cos (u y)$, which is a contradiction. Let $\mathscr{E}_{0}$ be the $\sigma$-algebra generated by $\{\cos (u x), x \in \mathbb{R}\}$. Then $\mathscr{E}_{0}$ is separable. Indeed, $\sigma(\{\cos u x, x \in \mathbb{R}\})=\sigma(\{\cos u x, x \in \mathbb{Q}\})$. The space $(\mathbb{R}, \mathscr{B}(\mathbb{R}))$ is a Blackwell space (cf. Dellacherie \& Meyer (1976) (Definition $3.24)$ ) and therefore if $\mathscr{E}$ is a separable sub- $\sigma$-algebra of $\mathscr{B}(\mathbb{R})$, then $\mathscr{B}(\mathbb{R})=\mathscr{E}$ if and only if $\mathscr{E}$ contains all the sets of the form $\{x\}, x \in \mathbb{R}$ (cf. Dellacherie \& Meyer (1976), Theorem 3.26). But $\{\cos (u x), x \in \mathbb{R}\}$ separates points and then we have $\{x\}=\bigcap_{u \in \mathbb{Q}}\{y: \cos (u y)=\cos (u x)\} \in \mathscr{E}_{0}$ so that $\mathscr{E}_{0}=\mathscr{B}(\mathbb{R})$. This implies that $\sigma(\mathscr{C})=\mathscr{F}^{Z}$ and we can conclude that $\mathscr{C}$ is dense in $L^{2}\left(\Omega, \mathscr{F}^{Z}, \mathbb{P}\right)$.

In a first step we consider the particular case in which the family $Z$ is composed by a unique element that we denote again $Z$. The general case will be obtained as a consequence of this one. The family of monomials in the random variable $Z$ is $\mathscr{K}_{1}:=\left\{Z^{n}, \quad n \geq 0\right\}$ and Assumption B.1 can be rephrased saying that there exists $c>0$ such that $\mathbb{E}[\exp (c|Z|)]<+\infty$. We put $\mathscr{P}_{1}:=\operatorname{Span}\left(\mathscr{K}_{1}\right)$ and let $\overline{\mathscr{P}}_{1}$ be the closure of $\mathscr{P}_{1}$ in $L^{2}\left(\Omega, \mathscr{F}^{Z}, \mathbb{P}\right)$. We are going to prove that the system $\mathscr{K}_{1}$ is total in $L^{2}\left(\Omega, \mathscr{F}^{Z}, \mathbb{P}\right)$. We begin with a preliminary result.

B.3 Lemma. Let $Z$ be a random variable and $c>0$ such that $\mathbb{E}\left[\mathrm{e}^{c|Z|}\right]<+\infty$. If $|u| \leq \frac{c}{4}$, then $Z^{l} \cos (u Z)$ belongs to $\overline{\mathscr{P}}_{1}$, for every $l \in \mathbb{N} \cup\{0\}$.

Proof. First we recall the series expansion of the cosine function:

$$
\lim _{m \rightarrow+\infty} \sum_{n=0}^{m}(-1)^{n} \frac{(u Z)^{2 n}}{(2 n) !}=\cos (u Z) .
$$

On the other side, we have

$$
\begin{aligned}
\left(Z^{l} \cos (u Z)\right. & \left.-Z^{l} \sum_{n=0}^{m}(-1)^{n} \frac{(u Z)^{2 n}}{(2 n) !}\right)^{2}=Z^{2 l}\left(\sum_{n=m+1}^{\infty}(-1)^{n} \frac{(u Z)^{2 n}}{(2 n) !}\right)^{2} \\
& \leq Z^{2 l}\left(\sum_{n=m+1}^{\infty} \frac{|u|^{2 n}|Z|^{2 n}}{(2 n) !}\right)^{2} \leq Z^{2 l}\left(\sum_{n=m+1}^{\infty}\left(\frac{\left|\frac{c}{4}\right|^{2 n}|Z|^{2 n}}{(2 n) !}\right)^{2}\right. \\
& \leq Z^{2 l}\left(\mathrm{e}^{\frac{c}{4}|Z|}\right)^{2}=Z^{2 l} \mathrm{e}^{\frac{c}{2}|Z|} .
\end{aligned}
$$

By Hölder's inequality we get

$$
\mathbb{E}\left[Z^{2 l} \mathrm{e}^{\frac{c}{2}|Z|}\right] \leq\left(\mathbb{E}\left[Z^{4 l}\right]\right)^{\frac{1}{2}}\left(\mathbb{E}\left[\mathrm{e}^{c|Z|}\right]\right)^{\frac{1}{2}}
$$

and because of $\mathbb{E}\left[\mathrm{e}^{c|Z|}\right]<+\infty$, the right-hand side in the previous expression is finite. Hence, applying the theorem of Lebesgue on dominated convergence, we can conclude that the polynomials $Z^{l} \sum_{n=0}^{m}(-1)^{n} \frac{(u Z)^{2 n}}{(2 n) !}$ converges in $L^{2}\left(\Omega, \mathscr{F}^{Z}, \mathbb{P}\right)$ to $Z^{l} \cos (u Z)$ and therefore $Z^{l} \cos (u Z) \in \overline{\mathscr{P}}_{1}$. 
Now we are ready to state and prove the following theorem:

B.4 Theorem. Let $Z$ be a random variable and $c>0$ such that $\mathbb{E}\left[\mathrm{e}^{c|Z|}\right]<+\infty$. The system $\mathscr{K}_{1}$ is total in $L^{2}\left(\Omega, \mathscr{F}^{Z}, \mathbb{P}\right)$.

Proof. From Lemma B.2, we know that the system $\mathscr{C}$ introduced in (B.3) is dense in $L^{2}\left(\Omega, \mathscr{F}^{Z}, \mathbb{P}\right)$. To conclude the proof it is enough to show that $\mathscr{C} \subseteq \overline{\mathscr{P}}_{1}$ and for this it is sufficient to verify that $\cos (u Z)$ belongs to $\overline{\mathscr{P}}_{1}$, for any $u \in \mathbb{R}$. Because of Lemma B.3, we have that $Z^{l} \cos (u Z)$ belongs to $\overline{\mathscr{P}}_{1}$ for every $l \in \mathbb{N} \cup\{0\}$ and $|u| \leq \frac{c}{4}$. We now assume that $Z^{l} \cos (u Z)$ belongs to $\overline{\mathscr{P}}_{1}$ for every $l \in \mathbb{N} \cup\{0\}$ and for every $u$ such that $|u| \leq k \frac{c}{4}$ and we prove that $Z^{l} \cos (u Z)$ belongs to $\overline{\mathscr{P}}_{1}$ for every $l \in \mathbb{N} \cup\{0\}$ and for every $u$ such that $|u| \leq(k+1) \frac{c}{4}$. We verify it for $u \geq 0$. The case $u<0$ is analogous. Let $u \in\left(v_{k}, v_{k+1}\right]$, with the notation $v_{j}=j \frac{c}{4}, j \in \mathbb{N}$. We consider $v:=u-v_{k}$. Then we get

$$
Z^{l} \cos (v Z) \cos \left(v_{k} Z\right)=\frac{1}{2} Z^{l}\left(\cos \left(\left(v+v_{k}\right) Z\right)+\cos \left(\left(v-v_{k}\right) Z\right)\right)
$$

and hence

$$
Z^{l} \cos (u Z)=2 Z^{l} \cos (v Z) \cos \left(v_{k} Z\right)-Z^{l} \cos \left(\left(v-v_{k}\right) Z\right) .
$$

Obviously, $0 \geq v-v_{k} \geq-v_{k}$, so $\left|v-v_{k}\right| \leq v_{k}$. By the induction hypothesis, this yields $Z^{l} \cos \left(\left(v-v_{k}\right) Z\right) \in \overline{\mathscr{P}}_{1}$. Thus, to verify that $Z^{l} \cos (u Z) \in \overline{\mathscr{P}}_{1}$, it is sufficient to prove that $Z^{l} \cos (v Z) \cos \left(v_{k} Z\right) \in \overline{\mathscr{P}}_{1}$. We have the pointwise convergence

$$
Z^{l} \cos (v Z) \cos \left(v_{k} Z\right)=\lim _{m \rightarrow \infty} \sum_{n=0}^{m}(-1)^{n} \frac{(v Z)^{2 n}}{(2 n) !} Z^{l} \cos \left(v_{k} Z\right) .
$$

Using the induction hypothesis, we obtain $Z^{2 n+l} \cos \left(v_{k} Z\right) \in \overline{\mathscr{P}}_{1}$ and hence

$$
\sum_{n=0}^{m}(-1)^{n} \frac{(v Z)^{2 n}}{(2 n) !} Z^{l} \cos \left(v_{k} Z\right) \in \overline{\mathscr{P}}_{1} .
$$

It remains to show the $L^{2}$-convergence of this series to $Z^{l} \cos (v Z) \cos \left(v_{k} Z\right)$. We estimate

$$
\begin{aligned}
& \left(Z^{l} \cos (v Z) \cos \left(v_{k} Z\right)-\sum_{n=0}^{m}(-1)^{n} \frac{(v Z)^{2 n}}{(2 n) !} Z^{l} \cos \left(v_{k} Z\right)\right)^{2} \\
& \leq\left(\sum_{n=m+1}^{\infty} \frac{(v Z)^{2 n}}{(2 n) !}\right)^{2} Z^{2 l}\left(\cos \left(v_{k} Z\right)\right)^{2} \leq Z^{2 l} \mathrm{e}^{\frac{c}{2}|Z|}
\end{aligned}
$$

and

$$
\mathbb{E}\left[Z^{2 l} \mathrm{e}^{\frac{c}{2}|Z|}\right] \leq\left(\mathbb{E}\left[Z^{4 l}\right]\right)^{\frac{1}{2}}\left(\mathbb{E}\left[\mathrm{e}^{\frac{c}{2}|Z|}\right]\right)^{\frac{1}{2}}<+\infty .
$$

Therefore we can apply the theorem of Lebesgue on dominated convergence and obtain $Z^{l} \cos (v Z) \cos \left(v_{k} Z\right) \in \overline{\mathscr{P}}_{1}$. This completes the proof of the theorem.

We now generalize the above result to the case of a family $Z=\left(Z_{\alpha}\right)_{\alpha \in I}$, where $I$ is an arbitrary set of indexes. Let $\mathscr{P}$ be the linear hull of $\mathscr{K}$, where $\mathscr{K}$ has been introduced in (B.1), and let $\overline{\mathscr{P}}$ be the closure of $\mathscr{P}$ in $L^{2}\left(\Omega, \mathscr{F}^{Z}, \mathbb{P}\right)$. 
B.5 Theorem. Let $Z=\left(Z_{\alpha}\right)_{\alpha \in I}$ be an arbitrary family of random variable satisfying Assumption B.1. The system $\mathscr{K}$ of monomials is total in $L^{2}\left(\Omega, \mathscr{F}^{Z}, \mathbb{P}\right)$.

Proof. By Lemma B.2, we know that the system $\mathscr{C}$ introduced in (B.3) is dense in $L^{2}\left(\Omega, \mathscr{F}^{Z}, \mathbb{P}\right)$. It is sufficient to verify that

$$
\cos \left(u_{1} Z_{\alpha_{1}}+\ldots+u_{m} Z_{\alpha_{m}}\right) \in \overline{\mathscr{P}}, \quad \alpha_{i}, \in I, u_{i}, \in \mathbb{R}, i=1, \ldots, m, \quad m \in \mathbb{N} .
$$

We put $X:=u_{1} Z_{\alpha_{1}}+\ldots+u_{m} Z_{\alpha_{m}}$. We have to show the existence of a $c>0$ such that $\mathbb{E}\left[\mathrm{e}^{c|X|}\right]<+\infty$. We have

$$
\mathbb{E}[\exp (c|X|)] \leq \mathbb{E}\left[\prod_{i=1}^{m} \mathrm{e}^{c\left|u_{i}\right|\left|Z_{\alpha_{i}}\right|}\right] \leq \mathbb{E}\left[\prod_{i=1}^{m} \exp \left(c\left(\max _{i=1, \ldots, m}\left|u_{i}\right|\right)\left|Z_{\alpha_{i}}\right|\right)\right]
$$

and so the choice of $c>0$ as $c:=\frac{\min _{i=1, \ldots, m} c_{\alpha_{i}}}{\max _{i=1, \ldots, m}\left|u_{i}\right|} c^{\prime}$, where $c_{\alpha_{i}}>0$ is such that $Z_{\alpha_{i}}$ satisfies (B.2), yields

$$
\mathbb{E}\left[\mathrm{e}^{c|X|}\right] \leq \mathbb{E}\left[\prod_{i=1}^{m} \exp \left(c^{\prime}\left(\min _{i=1, \ldots, m} c_{\alpha_{i}}\right)\left|Z_{\alpha_{i}}\right|\right)\right] \leq \mathbb{E}\left[\prod_{i=1}^{m} \exp \left(c^{\prime} c_{\alpha_{i}}\left|Z_{\alpha_{i}}\right|\right)\right] .
$$

It is well-known that if $y_{1}, \ldots, y_{n}$ are positive real number, then their geometric average can be estimated as it follows: $\left(\prod_{i=1}^{n} y_{i}\right)^{n} \leq \sum_{i=1}^{n} y_{i}$. Using this fact in (B.4), we get

$$
\begin{aligned}
\mathbb{E}\left[\mathrm{e}^{c|X|}\right] \leq \mathbb{E}\left[\prod_{i=1}^{m} \exp \left(c^{\prime} c_{\alpha_{i}}\left|Z_{\alpha_{i}}\right|\right)\right] & =\mathbb{E}\left[\left(\prod_{i=1}^{m} \exp \left(m c^{\prime} c_{\alpha_{i}}\left|Z_{\alpha_{i}}\right|\right)\right)^{\frac{1}{m}}\right] \\
& \leq \sum_{i=1}^{m} \mathbb{E}\left[\exp \left(m c^{\prime} c_{\alpha_{i}}\left|Z_{\alpha_{i}}\right|\right)\right] .
\end{aligned}
$$

This shows that if we choose $c^{\prime}:=\frac{1}{m}$ we obtain $\mathbb{E}\left[\mathrm{e}^{c|X|}\right]<+\infty$ with $c>0$ given by

$$
c:=\frac{\min _{i=1, \ldots, m} c_{\alpha_{i}}}{m \max _{i=1, \ldots, m}\left|u_{i}\right|} .
$$

Then we can apply Theorem B.4 to conclude that $\cos (X) \in \overline{\mathscr{P}_{1}(X)}$, where now $\mathscr{P}_{1}(X)$ denotes the set of polynomials in $X$. But obviously, $\mathscr{P}_{1}(X) \subseteq \mathscr{P}$ and so the claim is proven. 


\section{Bibliography}

D. Appelbaum. Lévy processes and stochastic calculus. Second edition. Cambridge University Press, 2009.

P. Baldi. Introduction to stochastic processes. Scuola Matem. Interuniv. Cortona, 2007. http://160.80.10.11/ processi/cortonalevy.pdf

H. Bauer. Measure and Integration Theory. De Gruytier studies in mathematics; 26.

R. Boel, P. Varaiya, E. Wong. Martingales on jump processes. I: Representation results. SIAM J. Control and Optimization, 13: 999-1021, 1975.

H. Brezis. Analyse fonctionelle-Téorie et Application. Masson Editeur; Paris, 1983.

J.M.C. Clark. The representation of functionals of Brownian motion by stochastic integrals. Ann. Math. Statist. 41, 1282-1295, 1970.

C.S. Chou, P.-A. Meyer. Sur la répresentation des martingales comme intégrales stochastique dans les processus ponctueles. Séminaire de Probabiltés IX, Lecture Notes in mathematics 465. Springer-Verlag, 1975.

M.A.H. Davis, P. Varaiya. The multiplicity of an increasing family of $\sigma$-fields. The Annals of Probability. Vol. 2, No. 5, 958-963, 1974.

M.A.H. Davis. The representation of martingales of jump processes. SIAM J. Control and Optimization, 14: 623-638, 1976.

C. Dellacherie, P.-A. Meyer. Probabilités et potential. I(1976) Hermann: Paris.

C. Dellacherie. Capacités et processus stocahstiques. Springer, Berlin 1972.

R.J. Elliott. Stochastic integrals for martingales of a jump process with partially accessible jump times. Z. Wahrscheinlichkeitstheorie ver. Geb, 36:213-266, 1976.

H.J. Engelbert, J. Hess. Stochastic integrals of continuous local martingales I. Mathematische Nachrichten 97: 325-343, 1980. 
H.J. Engelbert, J. Hess. Stochastic integrals of continuous local martingales II. Mathematische Nachrichten 100: 249-269, 1981.

I.I. Gihman, A.V. Skorohod. The theory of stochastic processes I. Springer-Verlag 1974.

S. He, J. Wang, J. Yan. Semimartingale Theory and Stochastic Calculus. Science Press, Beijing-New York, CRC Press INC.

K. Itô. Multiple Wiener integral. J. Math. Soc. Japan, 3: 157-169, 151.

J. Jacod. Calcul Stochastique et Problèmes de Martingales. Springer, Berlin Heidelberg 1979.

J. Jacod, A. Shiryaev. Limit Theorems for Stochastic Processes 2ed. Springer, 2000.

J. Jacod, M. Yor. Études des solutions extrémales et représentation intégrale des solutions pour certains problémes de martingales. Z. Wahrscheinlichkeitstheorie verw. Gebiete 38: 83-125, 1977.

Y. Kabanov. Representation of functionals of Wiener and Poisson processes as stochastic integrals. Theory of Probability and Its Applications, 18, 2, 1973.

Y. Kabanov. Integral representation for functionals of processes with independent increments. Theory of Probability and its Applications, 19, 4, 1974.

O. Kallenberg. Foundations of Modern Probability. Springer series in statistics. Probability and its applications. Springer-Verlag, 1997.

H. Kunita. Representation of martingales with jumps and applications to finance. Advanced Studies in Pure Mathematics 41, 2004 Stochastic Analysis and Related Topics pp. 209-232.

H. Kunita, S. Watanabe. On square integrable martingales. Nagoya Math. J. 30, 209-245, 1975.

D. Lepingle. Sur la represéntasion des sauts d'une martingale. Séminaire de Probabilités (Strasbourg) XI(1977). pg. 418-434. Springer Verlag.

P.A. Meyer. Probability and Potentials. Blaisdail Publishing Company. London, 1966.

D. Nualart, W. Schoutens. Chaotic and predictable representations for Lévy processes. Stochastic Processes and their Applications 90 (2000)109-122.

P. Protter. Stochastic Integration and Differential Equations. Springer, 2005; Edition 2.1 .

D. Revuz, M. Yor. Continuous Martingales and Brownian Motion. Springer Verlag, Berlin 1999; Edition 3.

K. Sato. Lévy processes and infinitely divisible distributions. Cambridge University Press 1999. 
M. Sharpe. General theory of Markov provesses. Pure and applied mathematics (Academic Press 1988): vol.133.

J. Wang. Some remarks on processes with independent increments. Séminaire de Probabilités (Strasbourg) XV(1981). pg. 627-631. Springer Verlag.

P. Wojtaszczyk. A mathematical introduction to wawelets. London Mathematical Society 1997. Student Text 37. 


\section{Ehrenwörtliche Erklärung}

Hiermit erkläre ich,

- dass mir die Promotionsordnung der Fakultät bekannt ist,

- dass ich die Dissertation selbst angefertigt habe, keine Textabschnitte oder Ergebnisse eines Dritten oder eigenen Prüfungsarbeiten ohne Kennzeichnung übernommen und alle von mir benutzten Hilfsmittel, persönliche Mitteilungen und Quellen in meiner Arbeit angegeben habe,

- dass ich die Hilfe eines Promotionsberaters nicht in Anspruch genommen habe und dass Dritte weder unmittelbar noch mittelbar geldwerte Leistungen von mir für Arbeiten erhalten haben, die im Zusammenhang mit dem Inhalt der vorgelegten Dissertation stehen,

- dass ich die Dissertation noch nicht als Prüfungsarbeit für eine staatliche oder andere wissenschaftliche Prüfung eingereicht habe.

Bei der Auswahl und Auswertung des Materials sowie bei der Herstellung des Manuskripts hat mich Prof. Dr. H.-J. Engelbert unterstützt.

Jena, den 17.07.2013

Paolo Di Tella 